\title{
Condições de contorno artificiais para a resolução numérica da equação da onda acústica em domínios ilimitados
}

\author{
Paula Neves de Araújo
}

\author{
DisSERTAÇÃO APRESENTADA \\ $\mathrm{AO}$ \\ Instituto De MatemáticA E EstatísticA \\ DA \\ Universidade DE SÃo PAUlo \\ PARA \\ OBTENÇÃO DO TÍTULO \\ $\mathrm{DE}$ \\ Mestre em CiÊnCIAS \\ Programa: Matemática Aplicada \\ Orientador: Prof. Dr. Saulo Rabello Maciel de Barros
}

Durante o desenvolvimento deste trabalho a autora recebeu auxílio financeiro da CAPES.

São Paulo, janeiro de 2020 


\section{Condições de contorno artificiais para a resolução numérica da equação da onda acústica em domínios ilimitados}

Esta versão da dissertação contém as correções e alterações sugeridas pela Comissão Julgadora durante a defesa da versão original do trabalho, realizada em 29/11/2019. Uma cópia da versão original está disponível no

Instituto de Matemática e Estatística da Universidade de São Paulo.

Comissão Julgadora:

- Prof. Dr. Saulo Rabello Maciel de Barros (orientador) - IME-USP

- Prof. Dr. André Pierro de Camargo - UFABC

- Prof. Dr. Ernani Vitillo Volpe - EP - USP 


\section{Agradecimentos}

Agradeço primeiramente a Deus pela vida, pelas pessoas que estiveram ao meu lado durante a elaboração deste trabalho e pela oportunidade de seguir fazendo o que amo ao longo dos últimos anos.

Agradeço aos meus pais, Iva e Monteiro, pelo apoio, por estarem sempre ao meu lado, me auxiliando em tudo que fosse possível, em especial nos momentos difíceis. Vocês são a melhor parte de mim e eu os amo mais do que posso dizer. Esse trabalho também é de vocês.

Agradeço à Patrícia, minha irmã, pela paciência e pelo apoio em mais essa jornada (e espero que venham muitas outras). Eu te amo e não conseguiria sem você.

Agradeço ao Gustavo, pela compreensão e por estar ao meu lado ao longo dos últimos anos. Eu te amo muito.

Agradeço também ao prof. Dr. Saulo Rabello Maciel de Barros por seu apoio, paciência e compreensão na orientação desta pesquisa, e aos demais membros da comissão julgadora deste trabalho, Prof. Dr. André Pierro de Camargo e Prof. Dr. Ernani Vitillo Volpe, pelas valiosas sugestões e contribuições para a elaboração desta versão.

Agradeço ainda aos integrantes do projeto de Software Technology for Modeling and Inversion (STMI) por sua disponibilidade em debater e contribuir com ideias de modo a enriquecer este trabalho.

Agradeço, por fim, a todos que me apoiaram, de perto ou de longe, ao longo da realização deste trabalho.

Agradeço à CAPES pelo apoio financeiro. 


\section{Resumo}

\section{ARAÚJO, P.N. Condições de contorno artificiais para a resolução numérica da equação}

da onda acústica em domínios ilimitados. 2019. 94 f. Dissertação (Mestrado) - Instituto de Matemática e Estatística, Universidade de São Paulo, São Paulo, 2019.

Neste trabalho apresentamos um estudo sobre condições de contorno utilizadas na resolução numérica da equação da onda acústica, com o objetivo de simular a propagação de ondas em meios ilimitados. Com este propósito, exploramos os aspectos teóricos dessa equação e também resultados relacionados ao processo de discretização e propriedades dos operadores obtidos, tais como estabilidade, consistência e convergência, e estudamos condições de contorno propostas por autores diversos: as camadas de amortecimento desenvolvidas por Cerjan e Sochacki; as condições de absorção sugeridas por Clayton, Engquist, Majda e Higdon; a condição de absorção híbrida (HABC) e a Perfectly Matched Layer (PML). A fim de comparar o desempenho dos métodos sugeridos, apresentamos testes com aplicação dos mesmos à resolução da equação da onda acústica em uma e duas dimensões espaciais e estudamos aspectos que podem afetar o desempenho dessas ferramentas. Ao fim do trabalho, trazemos ainda observações sobre a equação da onda elástica, sua dedução mediante hipóteses previamente estabelecidas e extensão das condições de contorno abordadas para esse tipo de equação.

Palavras-chave: Equação da onda acústica, métodos numéricos, condições de contorno, domínios ilimitados, camadas de absorção. 


\section{Abstract}

ARAÚJO, P. N. Artificial boundary conditions for numerical solution of the acoustic wave equation in unbounded domains. 2019. $94 \mathrm{f}$. Dissertação (Mestrado) - Instituto de Matemática e Estatística, Universidade de São Paulo, São Paulo, 2019.

In this work, we perform a study focused on boundary conditions for the numerical simulation of the acoustic wave equation, aiming at the simulation of wave propagation in infinite medium. With this purpose, we analyze properties of this equation and properties of the discretization, such as stability, consistency and convergence. We consider boundary treatments proposed by several authors: damping layers developed by Cerjan and Sochacki; absorbing boundary conditions suggested by Clayton, Engquist, Majda and Higdon; the hybrid absorbing boundary condition (HABC); and the Perfectly Matched Layer (PML). In order to compare the performance of these methods, we simulate them for the acoustic wave equation in one and two spatial dimensions, and we study some aspects that can affect their performance. We also include considerations about the elastic wave equation and its derivation, and the extension of the boundary conditions in this case.

Keywords: Acoustic wave equation, numerical methods, boundary conditions, unbounded domains, absorption layers. 


\section{Sumário}

$\begin{array}{ll}\text { Lista de Figuras } & \text { ix }\end{array}$

Lista de Tabelas $\quad$ xiii

$\begin{array}{ll}\text { Introdução } & 1\end{array}$

1 Equação da onda $\quad 5$

1.1 A equação da onda . . . . . . . . . . . . . . . . . . . . 5

2 Resolução numérica e condições artificiais de fronteira $\quad 11$

2.1 Discretização de segunda ordem por diferenças finitas . . . . . . . . . . . . . . . . . 11

2.2 Condições de absorção . . . . . . . . . . . . . . . . . . . . . 15

2.2 .1 Condição de Majda e Engquist . . . . . . . . . . . . . . . . . . 15

2.2 .2 Condição de Higdon . . . . . . . . . . . . . . . . . . . . . . . . . . 19

2.3 Camadas de absorção . . . . . . . . . . . . . . . . . . . . . 20

2.3 .1 Condição de Cerjan . . . . . . . . . . . . . . . . . . . 21

2.3.2 Condição de Sochacki . . . . . . . . . . . . . . . . . . . . . . . . 21

2.3.3 Perfectly Matched Layer (PML) . . . . . . . . . . . . . . . . . . . . . . . 23

2.3.4 Condição de absorção híbrida . . . . . . . . . . . . . . . . . . . 30

3 Resultados numéricos $\quad 33$

3.1 Equação da onda unidimensional - Exemplo 1 . . . . . . . . . . . . . 33

3.1 .1 Resultados para $\lambda=0.5 \ldots \ldots \ldots \ldots \ldots \ldots$

3.1.2 Efetividade das condições de contorno em relação a $\lambda \ldots$. . . . . . . . . . . 41

3.2 Equação da onda unidimensional - Exemplo $2 \ldots \ldots$. . . . . . . . . . . . 43

3.2.1 Resultados para $\Delta x=0.01$ e $\Delta t=0.005 \ldots \ldots \ldots \ldots \ldots$. . . . . 45

3.2.2 Desempenho das condições de contorno em relação ao refinamento da malha . 49

3.3 Equação da onda bidimensional - Exemplo 1 . . . . . . . . . . . . . . 50

3.3 .1 Resultados gerais . . . . . . . . . . . . . . . . . . 52

3.3.2 Desempenho das condições de contorno em relação à espessura da camada de amortecimento . . . . . . . . . . . . . . . . . . 5 59

3.4 Equação da onda bidimensional - Exemplo 2 . . . . . . . . . . . . . . 61

3.4 .1 Resultados gerais . . . . . . . . . . . . . . . . . . 62

3.4.2 Desempenho das condições de Cerjan, Sochacki e Higdon de primeira ordem em relação aos parâmetros . . . . . . . . . . . . . . . . . . . . . . . . 69

3.5 Equação da onda bidimensional - Exemplo $3 \ldots \ldots$. . . . . . . . . . . 70 
3.5.1 Resultados ............................ 71

4 Conclusões $\quad 75$

$\begin{array}{ll}\text { A Condições de contorno para a equação da onda elástica } & \mathbf{7 7}\end{array}$

A.1 Ondas sísmicas . . . . . . . . . . . . . . . . . . . . 77

A.2 Equação da onda sísmica no domínio contínuo . . . . . . . . . . . . . . . 78

A.3 Condições de contorno para a equação da onda elástica . . . . . . . . . . . . . . . . 82

$\begin{array}{ll}\text { Referências Bibliográficas } & 91\end{array}$ 


\section{Lista de Figuras}

2.1 Malha deslocada. . . . . . . . . . . . . . . . . . . . . . . . 13

2.2 Domínio para aplicação da HABC . . . . . . . . . . . . . . . . . . . 31

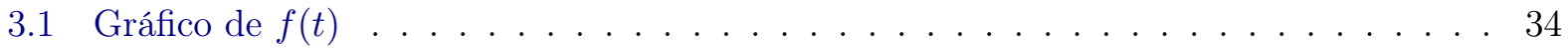

3.2 Solução de referência no decorrer do tempo para o primeiro exemplo com a equação da onda unidimensional. . . . . . . . . . . . . . . . . . . . 34

3.3 Condição de Dirichlet $u=0$ aplicada ao primeiro exemplo unidimensional. . . . . . . 36

3.4 Condição de Cerjan, com $\lambda=0.0125$, aplicada ao primeiro exemplo unidimensional. . 36

3.5 Condição A1 aplicada ao primeiro exemplo unidimensional. . . . . . . . . . . . . . 36

3.6 Condição HABC 1 aplicada ao primeiro exemplo unidimensional. . . . . . . . . . . . 37

3.7 Condição HABC 2 , com $M=2$ e $\alpha=1.5+0.07(L-M)$, aplicada ao primeiro exemplo unidimensional. . . . . . . . . . . . . . . . . . . 37

3.8 Condição de Higdon de primeira ordem, com $\alpha=\frac{\pi}{8}, a=b=0.5$, aplicada ao primeiro exemplo unidimensional. . . . . . . . . . . . . . . . 37

3.9 Condição de Higdon de segunda ordem, com $\alpha_{1}=\frac{\pi}{4}, \alpha_{2}=\frac{\pi}{3}, a_{1}=a_{2}=b_{1}=b_{2}=$ 0.5 , aplicada ao primeiro exemplo unidimensional. . . . . . . . . . . . . . 38

3.10 Condição PML para o sistema de primeira ordem, com $R=10^{-3}$, aplicada ao primeiro exemplo unidimensional. . . . . . . . . . . . . . . . . . 38

3.11 Condição PML para a equação de segunda ordem, com $R=10^{-7}$, aplicada ao primeiro exemplo unidimensional. . . . . . . . . . . . . . . . 38

3.12 Condição de Sochacki com amortecedor cúbico, $\operatorname{com} S=10^{7.5}$, aplicada ao primeiro exemplo unidimensional. . . . . . . . . . . . . . . . . . . . . . . . . . . . . . 39

3.13 Condição de Sochacki com amortecedor expoente, com $S=10$, aplicada ao primeiro exemplo unidimensional. . . . . . . . . . . . . . . . . . . . . . . . . . . . . 39

3.14 Condição de Sochacki com amortecedor linear, com $S=10^{3.25}$, aplicada ao primeiro exemplo unidimensional. . . . . . . . . . . . . . . . . . . . . . . . . . . . . . 39

3.15 Condição inicial para o segundo exemplo unidimensional. . . . . . . . . . . . . . . . . 43

3.16 Solução de referência no decorrer do tempo para o segundo exemplo com a equação da onda unidimensional. . . . . . . . . . . . . . . . . . . . . 44

3.17 Condição de Dirichlet $u=0$ aplicada ao segundo exemplo unidimensional. . . . . . . 45

3.18 Condição de Cerjan, com $\tilde{\lambda}=0.0001$, aplicada ao segundo exemplo unidimensional. . 45

3.19 Condição A1 aplicada ao segundo exemplo unidimensional. . . . . . . . . . . . . . . . 45

3.20 Condição HABC 1 aplicada ao segundo exemplo unidimensional. . . . . . . . . . . . 46 
3.21 Condição HABC 2, com $M=2$ e $\alpha=1.5+0.07(L-M)$, aplicada ao segundo exemplo unidimensional. . . . . . . . . . . . . . . . . . . . . . . . 46

3.22 Condição de Higdon de primeira ordem, com $\alpha=\frac{\pi}{8}, a=b=0.5$, aplicada ao segundo exemplo unidimensional. . . . . . . . . . . . . . . . . . . . . . . . . . 46

3.23 Condição de Higdon de segunda ordem, com $\alpha_{1}=\frac{\pi}{4}, \alpha_{2}=\frac{\pi}{3}, a_{1}=a_{2}=b_{1}=b_{2}=$ 0.5, aplicada ao segundo exemplo unidimensional. . . . . . . . . . . . . . . . . . . 46

3.24 Condição PML para o sistema de primeira ordem, com $R=10^{-4}$, aplicada ao segundo exemplo unidimensional. . . . . . . . . . . . . . . . . . . . . . . . . . . 47

3.25 Condição PML para a equação de segunda ordem, com $R=10^{-7}$, aplicada ao segundo exemplo unidimensional. . . . . . . . . . . . . . . . . . . . . . . . . . . . 47

3.26 Condição de Sochacki com amortecedor cúbico, com $S=10^{7.75}$, aplicada ao segundo exemplo unidimensional. . . . . . . . . . . . . . . . . . . . . . . . . 47

3.27 Condição de Sochacki com amortecedor expoente, com $S=10^{0.75}$, aplicada ao segundo exemplo unidimensional. . . . . . . . . . . . . . . . . . . . . . . . . . 47

3.28 Condição de Sochacki com amortecedor linear, com $S=10^{3.4}$, aplicada ao segundo exemplo unidimensional. . . . . . . . . . . . . . . . . . . . . . . . . . 48

3.29 Gráfico de $f(t)$. . . . . . . . . . . . . . . . . . . . . . . . . . . 50

3.30 Solução tomada como referência para o primeiro exemplo bidimensional no domínio de interesse. . . . . . . . . . . . . . . . . . . . . . . . . . . 51

3.31 Solução tomada como referência para o primeiro exemplo bidimensional no domínio estendido. . . . . . . . . . . . . . . . . . . . . . . . . 51

3.32 Solução tomada como referência para o primeiro exemplo bidimensional. . . . . . . . 52

3.33 Condição de Dirichlet $u=0$ aplicada ao primeiro exemplo bidimensional. . . . . . . . 52

3.34 Condição de Cerjan, com $\tilde{\lambda}=0.015$, aplicada ao primeiro exemplo bidimensional. . . 53

3.35 Condição A1 aplicada ao primeiro exemplo bidimensional. . . . . . . . . . . . . . . . 53

3.36 Condição A2 aplicada ao primeiro exemplo bidimensional. . . . . . . . . . . . . . . . 53

3.37 Condição de Higdon de primeira ordem, com $\alpha=\frac{\pi}{4}, a=b=0.5$, aplicada ao primeiro exemplo bidimensional. . . . . . . . . . . . . . . . . . . . . . . . . . . . . . 54

3.38 Condição de Higdon de segunda ordem, com $\alpha_{1}=0, \alpha_{2}=\frac{\pi}{4}, a_{1}=a_{2}=b_{1}=b_{2}=$ 0.5, aplicada ao primeiro exemplo bidimensional. . . . . . . . . . . . . . . . . . . . . 54

3.39 HABC original com a condição A1 aplicada ao primeiro exemplo bidimensional. . . . 54

3.40 HABC modificada com a condição A1, com $M=2, \alpha=1.5+0.07(L-M)$, aplicada ao primeiro exemplo bidimensional. . . . . . . . . . . . . . . . . . . . . . 55

3.41 HABC original com a condição A2 aplicada ao primeiro exemplo bidimensional. . . . 55

3.42 HABC modificada com a condição A2, com $M=2$ e $\alpha=1.5+0.07(L-M)$, aplicada ao primeiro exemplo bidimensional. . . . . . . . . . . . . . . . . . . . . . . 55

3.43 HABC original com a condição de Higdon de segunda ordem, $\alpha_{1}=0, \alpha_{2}=\frac{\pi}{4}$ e $a_{1}=a_{2}=b_{1}=b_{2}=0.5$, aplicada ao primeiro exemplo bidimensional. . . . . . . . . . 56

3.44 HABC modificada com a condição de Higdon de segunda ordem, com $M=2, \alpha=$ $1.5+0.07(L-M), \alpha_{1}=0, \alpha_{2}=\frac{\pi}{4}$ e $a_{1}=a_{2}=b_{1}=b_{2}=0.5$, aplicada ao primeiro exemplo bidimensional. . . . . . . . . . . . . . . . . . . . . . . . . . . . . . . . . . .

3.45 Condição PML para o sistema de primeira ordem, com $R=10^{-4}$, aplicada ao primeiro exemplo bidimensional. 
3.46 Condição PML para a equação de segunda ordem, com $R=10^{-7}$, aplicada ao primeiro exemplo bidimensional. . . . . . . . . . . . . . . . . . . . . . . . . . 57

3.47 Condição de Sochacki com amortecedor cúbico, com $S=1$, aplicada ao primeiro exemplo bidimensional. . . . . . . . . . . . . . . . . . . . . . . . . . . . 57

3.48 Condição de Sochacki com amortecedor expoente, com $S=0.5$, aplicada ao primeiro exemplo bidimensional. . . . . . . . . . . . . . . . . . . . . . . . . . . . . 57

3.49 Condição de Sochacki com amortecedor linear, com $S=10$, aplicada ao primeiro exemplo bidimensional. . . . . . . . . . . . . . . . . . . . . . . . . . . . . 58

3.50 Solução tomada como referência para o segundo exemplo bidimensional. . . . . . . . 62

3.51 Condição de Dirichlet $u=0$ aplicada ao segundo exemplo bidimensional. . . . . . . . 62

3.52 Condição de Cerjan, com $\tilde{\lambda}=0.0001$, aplicada ao segundo exemplo bidimensional. . . 62

3.53 Condição A1 aplicada ao segundo exemplo bidimensional. . . . . . . . . . . . . . . . . 63

3.54 Condição A2 aplicada ao segundo exemplo bidimensional. . . . . . . . . . . . . . . 63

3.55 Condição de Higdon de primeira ordem, com $\alpha=\frac{\pi}{8}, a=b=0.5$, aplicada ao segundo exemplo bidimensional. . . . . . . . . . . . . . . . . . . . . . . . 63

3.56 Condição de Higdon de segunda ordem, com $\alpha_{1}=0, \alpha_{2}=\frac{\pi}{4}, a_{1}=a_{2}=b_{1}=b_{2}=$ 0.5, aplicada ao segundo exemplo bidimensional . . . . . . . . . . . . . . . . . . 64

3.57 HABC original com a condição A1 aplicada ao segundo exemplo bidimensional. . . . 64

3.58 HABC modificada com a condição A1, com $M=2$ e $\alpha=1.5+0.07(L-M)$, aplicada ao segundo exemplo bidimensional. . . . . . . . . . . . . . . . . . . . . . . . . . . . . 64

3.59 HABC original com a condição A2 aplicada ao segundo exemplo bidimensional. . . . 65

3.60 HABC modificada com a condição A2, com $M=2$ e $\alpha=1.5+0.07(L-M)$, aplicada ao segundo exemplo bidimensional. . . . . . . . . . . . . . . . . . . . . . . 65

3.61 HABC original com a condição de Higdon de segunda ordem, com $\alpha_{1}=0, \alpha_{2}=\frac{\pi}{4} \mathrm{e}$ $a_{1}=a_{2}=b_{1}=b_{2}=0.5$, aplicada ao segundo exemplo bidimensional. . . . . . . . .

3.62 HABC modificada com a condição de Higdon de segunda ordem, $M=2, \alpha=1.5+$ $0.07(L-M), \alpha_{1}=0, \alpha_{2}=\frac{\pi}{4}$ e $a_{1}=a_{2}=b_{1}=b_{2}=0.5$, aplicada ao segundo exemplo bidimensional. . . . . . . . . . . . . . . . . . . . . . . . . . 66

3.63 Condição PML para o sistema de primeira ordem, com $R=10^{-4}$, aplicada ao segundo exemplo bidimensional. . . . . . . . . . . . . . . . . . . . . . . . . . 66

3.64 Condição PML para a equação de segunda ordem, com $R=10^{-2}$, aplicada ao segundo exemplo bidimensional. . . . . . . . . . . . . . . . . . . . . . . . . . . . . 66

3.65 Condição de Sochacki com amortecedor cúbico, com $S=10^{9}$, aplicada ao segundo exemplo bidimensional. . . . . . . . . . . . . . . . . . . . . . . . . . . . . 67

3.66 Condição de Sochacki com amortecedor expoente, com $S=10^{0.75}$, aplicada ao segundo exemplo bidimensional. . . . . . . . . . . . . . . . . . . . . . . . . . 67

3.67 Condição de Sochacki com amortecedor linear, com $S=10^{3.5}$, aplicada ao segundo exemplo bidimensional. . . . . . . . . . . . . . . . . . . . . . . . . . . 67

3.68 Gráfico de $f(t)$. . . . . . . . . . . . . . . . . . . . . . . . . . . . . . . . . . 70

3.69 Solução tomada como referência para o terceiro exemplo bidimensional. . . . . . . . 71

3.70 Condição A2 aplicada ao terceiro exemplo bidimensional. . . . . . . . . . . . . . . . . 72

3.71 Condição de Higdon de segunda ordem, com $\alpha_{1}=0, \alpha_{2}=\frac{\pi}{4}, a_{1}=a_{2}=b_{1}=b_{2}=$ 0.5, aplicada ao terceiro exemplo bidimensional. 
3.72 HABC modificada com a condição A1, com $M=2$ e $\alpha=1.5+0.07(L-M)$, aplicada ao terceiro exemplo bidimensional. . . . . . . . . . . . . . . . . 72

3.73 HABC modificada com a condição A2, com $M=2$ e $\alpha=1.5+0.07(L-M)$, aplicada ao terceiro exemplo bidimensional. . . . . . . . . . . . . . . . . . 73

3.74 HABC modificada com a condição de Higdon de segunda ordem, com $M=2, \alpha=$ $1.5+0.07(L-M), \alpha_{1}=0, \alpha_{2}=\frac{\pi}{4}$ e $a_{1}=a_{2}=b_{1}=b_{2}=0.5$, aplicada ao terceiro

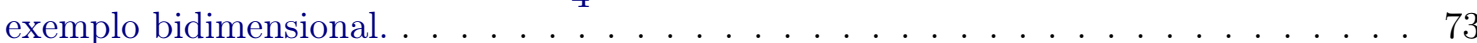

3.75 Condição PML para o sistema de primeira ordem, com $R=10^{-4}$, aplicada ao terceiro exemplo bidimensional. . . . . . . . . . . . . . . . . . 73

3.76 Condição PML para a equação de segunda ordem, com $R=10^{-7}$, aplicada ao terceiro exemplo bidimensional. . . . . . . . . . . . . . . . . . . . . . . 74 


\section{Lista de Tabelas}

3.1 Erros para o primeiro teste unidimensional. . . . . . . . . . . . . . . . . . 41

3.2 Erro para as condições de contorno com diversos valores de $\lambda . \ldots$. . . . . . . . . 42

3.3 Erros para o segundo teste unidimensional. . . . . . . . . . . . . . 48

3.4 Erro para as condições de contorno com diversos espaçamentos de malha. . . . . . . 49

3.5 Erros para o primeiro teste bidimensional. . . . . . . . . . . . . . 58

3.6 Erro para as camadas de amortecimento com diferentes valores de $L$. . . . . . . . . 60

3.7 Erros para o segundo teste bidimensional. . . . . . . . . . . . . . . 68

3.8 Erro para a condição de Cerjan com diversos valores de $\tilde{\lambda}$. . . . . . . . . . . . . . 69

3.9 Erro para a condição de Sochacki com amortecedor cúbico com diversos valores de $S$. 69

3.10 Erro para a condição de Sochacki com amortecedor expoente com diversos valores de

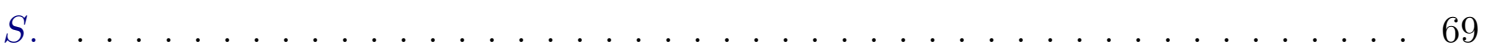

3.11 Erro para a condição de Sochacki com amortecedor linear com diversos valores de $S . \quad 69$

3.12 Erro para a condição de Higdon de primeira ordem com diversos valores de $\alpha$. . . . 69 


\section{Introdução}

Fenômenos ondulatórios estão presentes em diversas áreas da ciência, como é o caso das ondas acústicas, sísmicas e eletromagnéticas, por exemplo. Sua descrição matemática é feita por meio de equações conhecidas como equações de onda, que permitem prever o comportamento no decorrer do tempo dos eventos a que se referem.

O estudo da propagação de ondas teve início a partir da análise do movimento da corda de um violino, que pode ser modelada como uma linha infinitamente fina cujo movimento esteja contido em um plano. Este problema é abordado no primeiro capítulo deste trabalho e serve como base para o desenvolvimento de modelos que descrevem fenômenos mais complexos. Para descrever matematicamente a vibração da corda, recorre-se à $2^{\mathrm{a}}$ lei do movimento de Newton, que relaciona força e aceleração, e também à lei de Hooke, que afirma que a mudança no comprimento de uma mola é proporcional à força nela exercida. O obstáculo que surge, então, é o fato de que a lei de Newton pode ser aplicada a um número finito de pontos, resultando em um sistema de equações. A corda do violino possui infinitos pontos e, para resolver essa questão, os matemáticos da época descreveram a corda como um conjunto de massas próximas entre si, ligadas pela lei de Hooke, e fizeram com que o número de massas se tornasse arbitrariamente grande.

O raciocínio descrito anteriormente, bem como a verificação de que a forma de uma corda vibrando é a de uma curva senoidal, é atribuído a John Bernoulli e data de 1727. A aplicação de tal raciocínio visando a obtenção de equações ao invés de soluções diretas para o problema foi realizada por Jean Le Rond d'Alembert em 1746, considerando o fato de que, de acordo com a $2^{\text {a }}$ lei de Newton, a aceleração de um pequeno segmento (que é descrita por derivadas de segunda ordem no tempo) é proporcional às forças atuando nele. Por outro lado, a força atuando em um segmento é causada pela interação com segmentos vizinhos, e a noção de vizinhança se relaciona com a variação de espaço. Dessa forma, d'Alembert chegou à equação

$$
\frac{\partial^{2} u}{\partial t^{2}}=c^{2} \frac{\partial^{2} u}{\partial x^{2}}
$$

cujo significado é "A aceleração de um pequeno segmento de uma corda de violino é proporcional à média do deslocamento de segmentos vizinhos"([Ste12], p. 131).

Muitos problemas em âmbitos mais realistas demandam modelagens em duas e três dimensões, como a propagação de ondas na superfície do oceano, terremotos, etc. A extensão para esses casos é obtida por meio das mesmas considerações realizadas para o caso unidimensional e as equações obtidas são análogas.

Para o caso específico das aplicações em sismologia, a equação da onda pode ser utilizada no sentido de estudar terremotos e erupções de vulcões e, apesar de tais eventos serem causados por uma grande variedade de fatores, progressos têm sido alcançados nesse sentido. Outra aplicação da 
equação da onda a essa área é o processo de mapeamento da estrutura geológica de determinada região. Nesse caso, são causadas explosões na superfície e, de acordo com o sinal recebido de volta, procura-se obter informações sobre a constituição geológica do local. Esse processo é denominado Full Waveform Inversion (FWI) (ver, por exemplo, [Fic10]) e constitui a principal motivação para este trabalho.

Embora seja possível em alguns casos, a resolução analítica de equações de onda se mostra inviável para diversas aplicações e, por esse motivo, é frequentemente substituída pela resolução numérica, que fornece aproximações para as soluções de tais equações e é viabilizada pelo desenvolvimento tecnológico e a evolução das ferramentas computacionais que podem ser aplicadas para este fim.

A resolução numérica da equação da onda, assim como de outras equações diferenciais, envolve um processo de discretização do domínio e a obtenção de aproximações discretas para as derivadas em função dos valores da função incógnita nos pontos da malha, resultando em um modelo discreto que pode ser resolvido computacionalmente. As ferramentas computacionais permitem a realização de operações em um número finito de pontos, o que representa um obstáculo para o tratamento de fenômenos em domínios ilimitados. Nesse caso, faz-se necessário um truncamento do domínio, que deve ser feito de modo a aproximar ao máximo as características do modelo original.

Para que o truncamento do domínio não afete a solução obtida por meio da resolução numérica, são necessárias condições de contorno adequadas. A aplicação das condições de contorno geralmente é realizada com o objetivo de simular a propagação da onda para o exterior do domínio ou gerar o amortecimento da mesma, de modo que ocorra o mínimo de reflexão possível.

Neste trabalho estudamos algumas condições de contorno que foram desenvolvidas para a simulação da propagação de ondas em domínios ilimitados. Essas condições são divididas em condições de absorção e camadas de absorção (ou amortecimento). As condições de absorção consistem em prescrições realizadas diretamente nos contornos inseridos e geralmente são relacionadas ao comportamento da onda. As camadas de absorção são regiões acrescentadas em torno do domínio computacional nas quais a onda é amortecida, de modo a reduzir as reflexões no domínio de interesse.

De acordo com [Bér15], a necessidade de condições de contorno para tratamento de problemas formulados em domínios ilimitados surgiu na década de 1970, com o início do uso de computadores para o tratamento de equações por meio de diferenças finitas. No contexto de pulsos eletromagnéticos nucleares, as primeiras condições de contorno sugeridas foram as condições de radiação desenvolvidas por David E. Merewether em [Mer71] em 1971 para o caso bidimensional e estendidas para o caso tridimensional por Richard Holland em [Hol77] em 1977. Para a equação da onda acústica, à qual se dedica este trabalho, as condições de contorno mais utilizadas neste período foram as condições de absorção baseadas na One Way Wave Equation (OWWE) sugeridas por Engquist e Majda ([EM77]) em 1977 e por Higdon em 1986 em [Hig86]. Ainda na década de 1980, outras condições foram desenvolvidas por Liao et al. ([LHYY84]) em 1984, Keys ([Key85]) em 1985 e Reynolds ([Rey78]) em 1987.

Em 1985, Cerjan et al. sugeriram, em [CKKR85], o uso de camadas de amortecimento, e o mesmo foi feito por Sochacki et al. em 1987, em [SKG ${ }^{+}$87]. Já em 1994, a primeira Perfectly Matched Layer (PML) foi apresentada por Jean-Pierre Bérenger em seu trabalho [Ber94], destinado ao desenvolvimento desse tipo de condição de contorno para a propagação de ondas eletromagnéticas. 
Diversos trabalhos foram desenvolvidos neste sentido desde então, tanto para as equações de Maxwell ([CW94]), quanto para as equações da onda sísmica ([DKK15], [Fic10] e [FN03]) e da onda acústica ([Kim19], [Che19], [PM10], entre outros). A PML foi desenvolvida em formulações diversas, como a Anisotropic Perfectly Matched Layer (APML) proposta por Teixeira em [TC97], a Convolutional Perfectly Matched Layer (CPML), entre outras. O trabalho de Kristek, Moczo e Galis [KMG09] fornece um panorama geral acerca da PML e suas diversas formulações.

A obtenção de condições de contorno ideais para a simulação numérica da propagação de ondas em domínios ilimitados ainda é um problema em aberto. Algumas propostas recentes, como Hybrid Absorbing Boundary Condition (HABC ou hybrid ABC), por exemplo, ainda estão em desenvolvimento. A primeira HABC foi formulada por Liu [LS10] em 2010 e, posteriormente, diversos trabalhos foram desenvolvidos em relação a essa condição, como [LS18], por exemplo.

Neste trabalho abordamos condições de contorno para as equações da onda acústica em suas formas unidimensional e bidimensional e trazemos indicações acerca da aplicação de tais condições à equação da onda elástica, estendendo os resultados apresentados em [AB19], que traz resultados da aplicação de algumas das condições mencionadas à equação da onda acústica bidimensional.

O primeiro capítulo do trabalho versa sobre a equação da onda unidimensional, tratando de aspectos teóricos que nos permitem compreender os processos relacionados à resolução numérica. Neste capítulo, apresentamos também a equação bidimensional, para a qual está voltada a maioria dos procedimentos e testes descritos neste trabalho.

O segundo capítulo é destinado ao tratamento numérico das equações da onda e à descrição de condições de absorção e camadas de absorção. As condições que abordamos neste trabalho são as camadas de amortecimento propostas por Cerjan e Sochacki, as condições de absorção desenvolvidas por Engquist e Majda e Higdon, a PML e a HABC.

No terceiro capítulo trazemos exemplos da resolução das equações unidimensional e bidimensional da onda acústica com aplicação das condições de contorno abordadas e analisamos os resultados obtidos, comparando-os e observando aspectos que podem afetar o desempenho dessas ferramentas na simulação do fenômeno desejado.

Por fim, o quarto capítulo destina-se às conclusões acerca da pesquisa, considerações finais e perspectivas de pesquisas futuras. No apêndice A apresentamos considerações sobre o uso das condições de contorno estudadas para a equação da onda elástica. 
4 INTRODUÇÃO 


\section{Capítulo 1}

\section{Equação da onda}

Este capítulo é dedicado à equação da onda acústica de segunda ordem, principal objeto de estudo de nossa pesquisa, e a algumas de suas propriedades.

\subsection{A equação da onda}

Para realizar o estudo de propriedades da equação da onda unidimensional de segunda ordem, iniciamos considerando a equação unilateral unidimensional da onda, que possui a forma

$$
\begin{aligned}
u_{t}+c u_{x} & =0, c \in \mathbb{R}, x \in \mathbb{R} ; \\
u(x, 0) & =u_{0}(x),
\end{aligned}
$$

onde $u_{0}(x)$ é uma função previamente definida. Ao longo de curvas em que

$$
x-c t=\xi,
$$

sendo $\xi$ uma constante, temos, para uma solução de (1.1):

$$
\frac{d u}{d t}=\frac{d}{d t} u(c t+\xi, t)=c u_{x}+u_{t}=0 .
$$

Assim, $u$ é constante ao longo dessa curva, dependendo somente do valor de $\xi$. Dessa forma, a solução de (1.1) em um ponto $(x, t)$ corresponde à solução em $(x-c t, 0)$, ou seja,

$$
u(x, t)=u_{0}(x-c t) .
$$

Isso significa que, para cada $(x, t)$, a solução da equação será uma cópia da condição inicial deslocada para a direita, se $c>0$, ou para a esquerda, se $c<0$. É possível ver também que a solução no ponto $(x, t)$ depende da condição inicial calculada em $\xi=x-c t$; as curvas obtidas para cada $\xi$ são chamadas de curvas características. O parâmetro $c$ é dado em distância por tempo e, por este motivo, é conhecido como velocidade de propagação ao longo da característica.

A solução da equação (1.1) não leva em consideração quaisquer derivadas de $u_{0}$, o que sugere que podem ser aceitas soluções descontínuas para essa equação. Essa possibilidade se verifica para equações diferenciais parciais hiperbólicas em geral.

Em (1.1), consideramos $x \in \mathbb{R}$, mas também podemos considerar $x$ em um intervalo fechado de $\mathbb{R}$. Suponhamos $x \in[0,1]$. Nesse caso, a solução deve ser previamente determinada em $x=0$ de modo a estar definida para todo $t>0$. As condições adicionais necessárias nesse caso são chamadas de condições de contorno. Se impusermos as condições

$$
\begin{array}{r}
u(x, 0)=u_{0}(x) \\
u(0, t)=g(t),
\end{array}
$$

então a solução será dada por 


$$
u(x, t)=\left\{\begin{array}{l}
u_{0}(x-c t), \text { se } x-c t \geq 0, \\
g\left(t-\frac{x}{c}\right), \text { se } x-c t \leq 0 .
\end{array}\right.
$$

É importante observar que, ao longo de $x-c t=0$, a solução é determinada pelas duas equações, de modo que, se $u_{0} \neq g(0)$, haverá uma descontinuidade de salto nessa curva.

Um sistema da forma $u_{t}+A u_{x}+B u=F(x, t)$, no qual $u$ é uma função que retorna vetores, é hiperbólico se a matriz $A$ é diagonalizável com valores próprios reais $c_{i}$, que serão as velocidades características do sistema. Considere o sistema hiperbólico:

$$
\left(\begin{array}{c}
u_{t}^{1} \\
u_{t}^{2}
\end{array}\right)+\left(\begin{array}{cc}
a & b \\
b & a
\end{array}\right)\left(\begin{array}{c}
u_{x}^{1} \\
u_{x}^{2}
\end{array}\right)=0,0 \leq x \leq 1
$$

As velocidades características do sistema são $a+b$ e $a-b$. Consideremos $a, b>0$. Se $b<a$, então as duas famílias características se propagam para a direita, de modo que a solução deve ser dada em $x=0$. Se $a<b$, as famílias se propagam em direções opostas. Escrevendo o sistema (1.2) como

$$
\left(\begin{array}{c}
u_{t}^{1}+u_{t}^{2} \\
u_{t}^{1}-u_{t}^{2}
\end{array}\right)+\left(\begin{array}{cc}
a+b & 0 \\
0 & a-b
\end{array}\right)\left(\begin{array}{l}
u_{x}^{1}+u_{x}^{2} \\
u_{x}^{1}-u_{x}^{2}
\end{array}\right)=0,0 \leq x \leq 1,
$$

podemos observar que uma forma de determinar unicamente a solução é especificando $u^{1}+u^{2}$ em $x=0$ e $u^{1}-u^{2}$ em $x=1$, uma vez que essas são as variáveis correspondentes às equações cujas velocidades de propagação são positiva e negativa, respectivamente. Podemos especificar essas variáveis como segue:

$$
\begin{aligned}
& u^{1}+u^{2}=\alpha_{0}\left(u^{1}-u^{2}\right)+\beta_{0} \\
& u^{1}-u^{2}=\alpha_{1}\left(u^{1}+u^{2}\right)+\beta_{1},
\end{aligned}
$$

com $\alpha_{0}$ e $\alpha_{1}$ sendo funções de $t$ ou constantes.

Uma condição de contorno é dita bem-posta se determina unicamente a solução e isso ocorre se, e somente se, a mesma equivale a (1.4). Podemos observar que a variável $u^{1}+u^{2}$ é definida em termos de $u^{1}-u^{2}$ e vice-versa, o que indica que as variáveis não podem ser especificadas de forma mutuamente independente.

Consideremos agora a equação da onda unidimensional de segunda ordem

$$
\begin{aligned}
& u_{t t}-c^{2} u_{x x}=0, \\
& u(0, x)=f(x), \\
& u_{t}(0, x)=g(x)
\end{aligned}
$$

com $c$ constante. A análise da equação acima pode ser feita a partir da mudança de coordenadas

$$
x+c t=\xi, x-c t=\eta,
$$

de acordo com a qual a equação (1.5) é escrita como

$$
u_{\xi \eta}=0 .
$$

Assim, a função $u_{\xi}$ é uma função independente de $\eta$, ou seja,

$$
u_{\xi}=\alpha(\xi)
$$

de modo que

$$
u=\int \alpha(\xi) d \xi+G(\eta)=F(\xi)+G(\eta)
$$


Nas variáveis originais, $u=F(x+c t)+G(x-c t)$. Utilizando as condições iniciais $u(x, 0)$ e $u_{t}(x, 0)$, obtemos as relações

$$
\begin{aligned}
u(x, 0) & =F(x)+G(x)=f(x) \\
u_{t}(x, 0) & =c F^{\prime}(x)-c G^{\prime}(x)=g(x) .
\end{aligned}
$$

Assim,

$$
\begin{aligned}
F^{\prime}(x) & =\frac{c f^{\prime}(x)+g(x)}{2 c} \\
G^{\prime}(x) & =\frac{c f^{\prime}(x)-g(x)}{2 c}
\end{aligned}
$$

ou, equivalentemente,

$$
\begin{aligned}
& F(x)=\frac{f(x)}{2}+\frac{1}{2 c} \int_{0}^{x} g(\xi) d \xi+\delta \\
& G(x)=\frac{f(x)}{2}-\frac{1}{2 c} \int_{0}^{x} g(\xi) d \xi+\varepsilon
\end{aligned}
$$

Pela equação (1.8), $\delta+\varepsilon=0$. Portanto,

$$
u(x, t)=\frac{f(x+c t)+f(x-c t)}{2}+\frac{1}{2 c} \int_{x-c t}^{x+c t} g(\xi) d \xi .
$$

Logo, a solução $u(x, t)$ é determinada de forma única pelas funções $f$ e $g$. Além disso, o valor da solução em um ponto dado depende apenas de $f$ calculada em $x-c t$ e $x+c t$ e de $g$ no intervalo $[x-c t, x+c t]$. O intervalo $[x-c t, x+c t]$ é o domínio de dependência das condições iniciais da solução $u$ no ponto $(x, t)$. Os pontos $(x-c t, 0)$ e $(x+c t, 0)$ são as intersecções das curvas características que passam por $(x, t)$ com inclinações $\frac{1}{c} \mathrm{e}-\frac{1}{c}$ em relação ao eixo $t$. Reciprocamente, define-se o domínio de influência nas condições iniciais como o conjunto de pontos delimitado pelas curvas características passando por $(y, 0)$ nos quadrantes superiores do plano $x t$, para determinado valor de $y$.

A resolução analítica da equação da onda em dimensões mais altas exige ferramentas mais elaboradas. A equação n-dimensional da onda é escrita como

$$
u_{t t}-c^{2} \Delta u=0
$$

onde $\Delta u$ denota o Laplaciano da função $u$.

Neste trabalho, abordamos com maior atenção o caso $n=2$, embora o caso unidimensional seja usado na descrição de conceitos relacionados ao tratamento numérico. O estudo analítico das equações com $n>1$ pode ser encontrado em [Joh82], bem como da equação não homogênea

$$
u_{t t}-c^{2} \Delta u=w(x, t) .
$$

Outras informações sobre a equação da onda e problemas hiperbólicos em geral podem ser encontradas em [Eva10], [BWZ10] e [GKO95].

Conforme mencionamos anteriormente, o tratamento da equação da onda em domínios ilimitados demanda o uso de condições de contorno. A fim de motivar a discussão de condições de contorno mais elaboradas, abordaremos primeiramente as condições de Dirichlet e Neumann. Considere a 
equação da onda bidimensional de segunda ordem, definida para $x \in \mathbb{R}$ e $y \in \mathbb{R}$ :

$$
\begin{aligned}
& \frac{\partial^{2} u}{\partial t^{2}}=c^{2}\left(\frac{\partial^{2} u}{\partial x^{2}}+\frac{\partial^{2} u}{\partial y^{2}}\right)+F(x, y, t) \\
& u(x, y, 0)=f_{1}(x, y) \\
& \frac{\partial u}{\partial t}(x, y, 0)=f_{2}(x, y),
\end{aligned}
$$

Por simplicidade, consideramos $c=1$. Como a equação (1.12) não pode ser analisada numericamente em todo o domínio, podemos considerar um domínio $[-a, a] \times[-b, b]$ e acrescentar à equação dada uma das seguintes condições homogêneas:

$$
\begin{aligned}
& u( \pm a, y, t)=0, u(x, \pm b, t)=0 \\
& \frac{\partial u}{\partial x}( \pm a, y, t)=0, \frac{\partial u}{\partial y}(x, \pm b, t)=0 .
\end{aligned}
$$

A condição (1.15) é a condição de Dirichlet e (1.16) é a condição de Neumann e as mesmas geram problemas bem-postos. De fato, se $F=0$ no problema (1.12) com as condições de contorno (1.15)-(1.16), o problema não será bem-posto se pudermos encontrar como solução para o mesmo uma onda simples, não trivial, do tipo

$$
u=e^{s t+i \omega y} \varphi(x),
$$

$\operatorname{com}\|\varphi(\cdot)\|<\infty, \operatorname{Re}(s)>0$. Essa condição decorre da condição de Lopatinsky (estabelecida para sistemas de primeira ordem), e sua demonstração pode ser encontrada em [GKO95].

A verificação de que um problema é bem-posto é feita por meio da busca por soluções que correspondam à condição dada. Para verificar a existência de tais soluções, introduzimos na equação (1.12) a função $u$ dada anteriormente, obtendo a seguinte equação diferencial ordinária:

$$
\begin{aligned}
& e^{s t+i \omega y} \varphi_{x x}(x)-\omega^{2} e^{s t+i \omega y} \varphi(x)=s^{2} e^{s t+i \omega y} \varphi(x) \\
& \Rightarrow \varphi_{x x}(x)-\varphi(x)\left(\omega^{2}-s^{2}\right)=0 .
\end{aligned}
$$

As condições de contorno em $x$ para a EDO acima são as seguintes:

$$
\begin{aligned}
& \varphi(0)=0 \\
& \varphi_{x}(0)=0
\end{aligned}
$$

A solução geral de (1.18) é

$$
\varphi(x)=\sigma_{1} e^{k x}+\sigma_{2} e^{-k x},
$$

onde $\pm k$ são as raízes do polinômio característico $k^{2}-\left(s^{2}+\omega^{2}\right)=0$, ou seja, $k= \pm \sqrt{s^{2}+\omega^{2}}$. Conforme demonstrado em [GKO95] (lema 9.1.3, p. 289), existe $\delta>0$ tal que $|\operatorname{Re}(k)|>\delta|\operatorname{Re}(s)|$, o que indica que, para obtermos $\varphi(x) \in \mathcal{L}^{2}$, devemos ter $\sigma_{1}=0$.

Aplicando a solução geral (1.21) à condição inicial (1.15), temos

$$
\sigma_{2}=0 \Rightarrow \varphi \equiv 0 .
$$

Logo, como a solução encontrada é trivial, a condição (1.15) leva a um problema bem-posto.

Para a condição (1.16), obtemos

$$
k \sigma_{2}=0 \Rightarrow k=0 \text { ou } \sigma_{2}=0 .
$$

Como $\operatorname{Re}(k)>0$, a primeira opção não se aplica. Se $\sigma_{2}=0$, temos novamente $\varphi \equiv 0$ e, portanto, o problema também é bem-posto para a condição (1.16). 
Apesar de gerarem problemas bem-postos, as condições de Dirichlet e Neumann não são adequadas para simulação da propagação de ondas em domínios ilimitados, pois geram reflexões das ondas propagadas quando as mesmas atingem os contornos introduzidos artificialmente.

Consideremos que a solução exata da equação (1.5) com as condições dadas seja uma onda se propagando para a direita da forma $u=e^{\imath(\omega t-k x)}$, onde $\omega$ é a frequência angular e $k$ é o número de onda. A solução de (1.5) para $x \leq a$ é dada por

$$
u=e^{\imath(\omega t-k x)}+R e^{\imath(\omega t+k x)} .
$$

A solução acima é composta por duas ondas se propagando em direções opostas. Na ausência dos contornos artificiais, o comportamento esperado para o fenômeno consiste em ondas se propagando apenas para a direita, o que resultaria em $R=0$. No entanto, substituindo (1.22) na condição (1.15), temos

$$
\begin{aligned}
& e^{\imath(\omega t-k x)}+R e^{\imath(\omega t+k x)}=0, x=a \\
\Rightarrow & R=-\frac{e^{\imath(\omega t-k x)}}{e^{\imath(\omega t+k x)}}=-e^{\imath(-2 k x)} .
\end{aligned}
$$

Consequentemente, $|R|=1$ e isso significa que a amplitude da onda refletida pela condição de contorno em $x=a$ é a mesma da onda incidente. Do mesmo modo, para a condição (1.16):

$$
\begin{aligned}
& -k e^{\imath(\omega t-k x)}+R k e^{\imath(\omega t+k x)}=0 \\
\Rightarrow & R=\frac{e^{\imath(\omega t-k x)}}{e^{\imath(\omega t+k x)}}=e^{\imath(-2 k x)} \\
\Rightarrow & |R|=1 .
\end{aligned}
$$

O efeito produzido pelo uso dessas condições de contorno é indesejável, uma vez que a solução resultante inclui ondas se propagando no sentido contrário ao esperado e, portanto, não pode representar a solução esperada para a propagação no domínio ilimitado. Assim, faz-se necessário o uso de condições de contorno que minimizem esse efeito, como as que abordamos no próximo capítulo. 


\section{Capítulo 2}

\section{Resolução numérica e condições artificiais de fronteira para a equação da onda}

Neste capítulo, introduzimos conceitos relacionados ao tratamento numérico de equações diferenciais e à discretização por diferenças finitas, ferramenta que utilizamos para representação das equações em domínios discretos, e apresentamos condições artificiais de contorno que podem ser utilizadas para que o truncamento do domínio seja realizado de forma adequada ao fenômeno que se deseja observar. As equações com as quais trabalhamos são a equação unidimensional da onda acústica no domínio $\Omega_{1}=\left[x_{I}, x_{F}\right]$ e a equação bidimensional no domínio $\Omega_{2}=\left[x_{I}, x_{F}\right] \times\left[y_{I}, y_{F}\right]$.

\subsection{Discretização de segunda ordem por diferenças finitas}

Consideremos o esquema de segunda ordem padrão para a equação (1.5), no qual $v_{i}^{n}$ representa a solução calculada no ponto $x_{i}$ no tempo $t_{n}$ :

$$
\frac{v_{i}^{n+1}-2 v_{i}^{n}+v_{i}^{n-1}}{\Delta t^{2}}=c^{2} \frac{v_{i+1}^{n}-2 v_{i}^{n}+v_{i-1}^{n}}{\Delta x^{2}}
$$

Para verificar as condições de estabilidade do esquema (2.1), utilizamos a análise de von Neumann. Inicialmente, observamos a necessidade de satisfazer a condição de Courant-Friedrichs-Lewy, ou condição de CFL, que requer que o domínio de dependência da equação esteja, ao menos assintoticamente, contido no domínio de dependência numérico do método. Para este esquema, isto se traduz na condição $|\lambda| \leq 1$, onde $\lambda=c \frac{\Delta t}{\Delta x}$. A constante $\lambda$ é denominada constante de $C F L$ e frequentemente utilizada como critério de estabilidade para esquemas de diferenças finitas. Mais detalhes acerca dos resultados desenvolvidos por Courant, Friedrichs e Lewy podem ser encontrados em seu trabalho original [CFL28]. Através da análise de von Neumann, verificamos a seguir que a condição $|\lambda| \leq 1$ garante a estabilidade do esquema.

Para o método (2.1), temos a seguinte expressão para o fator de amplificação $g$ para cada modo de Fourier $e^{\imath k x}$ :

$$
g-2+g^{-1}=-4 \lambda^{2} \operatorname{sen}^{2} \frac{\theta}{2}
$$

onde $\theta=k \Delta x$.

A aplicação da análise de von Neumann estabelece que os fatores de amplificação $g$ pelos quais serão multiplicados os modos de Fourier $e^{\imath k x}$ devem ser tais que $|g| \leq 1$. Desenvolvendo a expressão para $g$, obtemos

$$
\left(g^{\frac{1}{2}}-g^{-\frac{1}{2}}\right)^{2}=-4 \lambda^{2} \operatorname{sen}^{2} \frac{\theta}{2}
$$




$$
\begin{aligned}
& \Rightarrow g^{\frac{1}{2}}-g^{-\frac{1}{2}}= \pm \lambda 2 \imath \operatorname{sen} \frac{\theta}{2} \\
& \Rightarrow g-1= \pm 2 \imath \lambda \operatorname{sen} \frac{\theta}{2} g^{\frac{1}{2}} \\
& \Rightarrow g \pm 2 \imath \lambda \operatorname{sen} \frac{\theta}{2} g^{\frac{1}{2}}-1=0
\end{aligned}
$$

Resolvendo essa equação para $g^{\frac{1}{2}}$, temos que $g^{\frac{1}{2}}=\left(\sqrt{1-\lambda^{2} \operatorname{sen}^{2} \frac{\theta}{2}} \pm \imath \lambda \operatorname{sen} \frac{\theta}{2}\right)$, ou seja,

$$
g=\left(\sqrt{1-\lambda^{2} \operatorname{sen}^{2} \frac{\theta}{2}} \pm \imath \lambda \operatorname{sen} \frac{\theta}{2}\right)^{2}=1-2 \lambda^{2} \operatorname{sen}^{2} \frac{\theta}{2} \pm 2 \imath \lambda \operatorname{sen} \frac{\theta}{2} \sqrt{1-\lambda^{2} \operatorname{sen}^{2} \frac{\theta}{2}} .
$$

Se $|\lambda| \leq 1, \sqrt{1-\lambda^{2} \operatorname{sen}^{2} \frac{\theta}{2}} \in \mathbb{R}$ e, portanto,

$$
\begin{gathered}
|g|^{2}=\left(1-2 \lambda^{2} \operatorname{sen}^{2} \frac{\theta}{2}\right)^{2}+4 \lambda^{2} \operatorname{sen}^{2} \frac{\theta}{2}\left(1-\lambda^{2} \operatorname{sen}^{2} \frac{\theta}{2}\right) \\
\Rightarrow|g|^{2}=1-4 \lambda^{2} \operatorname{sen}^{2} \frac{\theta}{2}+4 \lambda^{4} \operatorname{sen}^{4} \frac{\theta}{2}+4 \lambda^{2} \operatorname{sen}^{2} \frac{\theta}{2}-4 \lambda^{4} \operatorname{sen}^{4} \frac{\theta}{2}=1 .
\end{gathered}
$$

Assim, o método é estável pela condição estabelecida para $g$ para $|\lambda| \leq 1$ e, como também é consistente com a equação diferencial, o esquema de diferenças finitas (2.1) é convergente de ordem 2, de acordo com o teorema de Lax-Richtmyer (veja, por exemplo, [Str04]).

Considerando a equação da onda bidimensional de segunda ordem

$$
\frac{\partial^{2} u}{\partial t^{2}}=c^{2}\left(\frac{\partial^{2} u}{\partial x^{2}}+\frac{\partial^{2} u}{\partial y^{2}}\right)
$$

podemos realizar um tratamento análogo ao caso unidimensional, utilizando passos $\Delta x$ e $\Delta y$ nas discretizações das derivadas espaciais, que podem ser feitas separadamente. Assim, a discretização da equação (2.2) resulta na equação (2.1) acrescida das parcelas referentes à discretização na variável $y$. Portanto, para a equação $(2.2)$, temos

$$
\frac{v_{i, j}^{n+1}-2 v_{i, j}^{n}+v_{i, j}^{n-1}}{\Delta t^{2}}=c^{2} \frac{v_{i+1, j}^{n}-2 v_{i, j}^{n}+v_{i-1, j}^{n}}{\Delta x^{2}}+c^{2} \frac{v_{i, j+1}^{n}-2 v_{i, j}^{n}+v_{i, j-1}^{n}}{\Delta y^{2}} .
$$

Para obtenção de uma condição de estabilidade análoga à verificada para o caso unidimensional, utilizamos a análise de von Neumann para dimensões mais altas. Nesse caso, mostraremos que se $\lambda_{1}^{2}+\lambda_{2}^{2} \leq 1$, onde $\lambda_{1}=c \frac{\Delta t}{\Delta x}$ e $\lambda_{2}=c \frac{\Delta t}{\Delta y}$, o método é estável. O polinômio de amplificação $g$ para (2.3) é dado por

$$
\begin{aligned}
& \left(g^{\frac{1}{2}}-g^{-\frac{1}{2}}\right)^{2}=-4 c^{2} \Delta t^{2}\left(\frac{\operatorname{sen}^{2} \frac{\theta}{2}}{\Delta x^{2}}+\frac{\operatorname{sen}^{2} \frac{\phi}{2}}{\Delta y^{2}}\right) \\
& \Rightarrow g^{\frac{1}{2}}-g^{-\frac{1}{2}}=2 \imath c \Delta t\left(\frac{\operatorname{sen}^{2} \frac{\theta}{2}}{\Delta x^{2}}+\frac{\operatorname{sen}^{2} \frac{\phi}{2}}{\Delta y^{2}}\right)^{\frac{1}{2}} \\
& \Rightarrow g-2 \imath c \Delta t\left(\frac{\operatorname{sen}^{2} \frac{\theta}{2}}{\Delta x^{2}}+\frac{\operatorname{sen}^{2} \frac{\phi}{2}}{\Delta y^{2}}\right)^{\frac{1}{2}} g^{\frac{1}{2}}-1=0 .
\end{aligned}
$$

A resolução da equação anterior para $g$ resulta em 


$$
g=\left[\imath c \Delta t\left(\frac{\operatorname{sen}^{2} \frac{\theta}{2}}{\Delta x^{2}}+\frac{\operatorname{sen}^{2} \frac{\phi}{2}}{\Delta y^{2}}\right)^{\frac{1}{2}} \pm \sqrt{1-\Delta t^{2} c^{2}\left(\frac{\operatorname{sen}^{2} \frac{\theta}{2}}{\Delta x^{2}}+\frac{\operatorname{sen}^{2} \frac{\phi}{2}}{\Delta y^{2}}\right)}\right]^{2}
$$

Assim, se $\lambda_{1}^{2}+\lambda_{2}^{2} \leq 1$, temos que $\sqrt{1-\Delta t^{2} c^{2}\left(\frac{\operatorname{sen}^{2} \frac{\theta}{2}}{\Delta x^{2}}+\frac{\operatorname{sen}^{2} \frac{\phi}{2}}{\Delta y^{2}}\right)} \in \mathbb{R}$ para quaisquer $\theta$ e $\phi$ (ou seja, para quaisquer frequências) e, dessa forma,

$$
|g|=\lambda_{1}^{2} \operatorname{sen}^{2} \frac{\theta}{2}+\lambda_{2}^{2} \operatorname{sen}^{2} \frac{\phi}{2}+1-\lambda_{1}^{2} \operatorname{sen}^{2} \frac{\theta}{2}-\lambda_{2}^{2} \operatorname{sen}^{2} \frac{\phi}{2}=1 .
$$

Portanto, a discretização (2.3) é estável e, por ser consistente com a equação diferencial, o método também é convergente.

Podemos ainda considerar a equação da onda unidimensional na forma de sistema de primeira ordem como segue:

$$
\begin{aligned}
& \frac{\partial u}{\partial t}=c \frac{\partial v}{\partial x} \\
& \frac{\partial v}{\partial t}=c \frac{\partial u}{\partial x} .
\end{aligned}
$$

Para esse sistema, utilizamos o seguinte esquema de diferenças finitas:

$$
\begin{aligned}
\frac{u_{i}^{n+1}-u_{i}^{n}}{\Delta t} & =c \frac{v_{i+\frac{1}{2}}^{n+\frac{1}{2}}-v_{i-\frac{1}{2}}^{n+\frac{1}{2}}}{\Delta x} \\
\frac{v_{i+\frac{1}{2}}^{n+\frac{1}{2}}-v_{i+\frac{1}{2}}^{n-\frac{1}{2}}}{\Delta t} & =c \frac{u_{i+1}^{n}-u_{i}^{n}}{\Delta x}
\end{aligned}
$$

Esse esquema é obtido através do uso de diferenças centradas em uma malha deslocada. Uma representação gráfica da malha considerada é a seguinte:

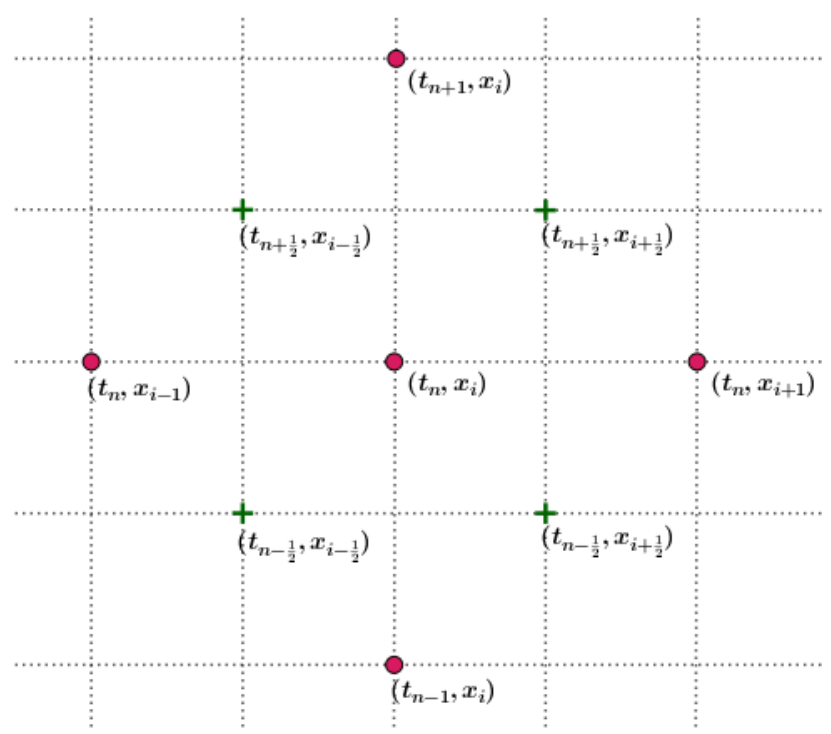

Figura 2.1: Malha deslocada.

Para obtermos a matriz de amplificação do esquema (2.5), observamos, a partir da aplicação dos operadores de diferenças no espaço aos modos de Fourier $e^{\imath k x}$, que 


$$
\left(\begin{array}{cc}
1 & -\imath a \\
0 & 1
\end{array}\right)\left(\begin{array}{c}
u^{n+1} \\
v^{n+\frac{1}{2}}
\end{array}\right)=\left(\begin{array}{cc}
1 & 0 \\
\imath a & 1
\end{array}\right)\left(\begin{array}{c}
u^{n} \\
v^{n-\frac{1}{2}}
\end{array}\right)
$$

onde $a=\frac{2 c \Delta t}{\Delta x} \operatorname{sen} \frac{\theta}{2}, \operatorname{com} \theta=k \Delta x$ e $\left(\begin{array}{c}u^{n} \\ v^{n-\frac{1}{2}}\end{array}\right)=e^{\imath k x}\left(\begin{array}{c}\alpha \\ \beta\end{array}\right)$.

Resulta que

$$
\left(\begin{array}{c}
u^{n+1} \\
v^{n+\frac{1}{2}}
\end{array}\right)=\left(\begin{array}{cc}
1-a^{2} & \imath a \\
\imath a & 1
\end{array}\right)\left(\begin{array}{c}
u^{n} \\
v^{n-\frac{1}{2}}
\end{array}\right)
$$

A matriz $G=\left(\begin{array}{cc}1-a^{2} & \imath a \\ \imath a & 1\end{array}\right)$ é a matriz de amplificação do esquema de diferenças, que relaciona o vetor $\left(\begin{array}{c}u^{n} \\ v^{n-\frac{1}{2}}\end{array}\right)$ ao vetor $\left(\begin{array}{c}u^{n+1} \\ v^{n+\frac{1}{2}}\end{array}\right)$.

A condição para que o esquema correspondente seja estável é que $\left\|G^{n}\right\|$ seja limitado. Os autovalores de $G$ nesse caso são as raízes de $\mu^{2}-\mu\left(2-a^{2}\right)+1=0$. Tais raízes são

$$
\mu=\frac{2-a^{2}}{2} \pm \frac{|a| \sqrt{a^{2}-4}}{2} .
$$

Se a condição de CFL $|\lambda|=\frac{c \Delta t}{\Delta x} \leq 1$ é satisfeita, então $a^{2} \leq 4$ e $\sqrt{a^{2}-4}$ é imaginário. Então

$$
|\mu|^{2}=\frac{a^{4}-4 a^{2}+4}{4}+\frac{\left(-a^{4}+4 a^{2}\right)}{4}=1 .
$$

Assim, o método é estável e, por ser consistente de ordem 2 com a equação, é também convergente de ordem 2.

Consideramos, então, o caso bidimensional

$$
\begin{aligned}
& \frac{\partial u}{\partial t}=c\left(\frac{\partial v}{\partial x}+\frac{\partial w}{\partial y}\right) \\
& \frac{\partial v}{\partial t}=c \frac{\partial u}{\partial x} \\
& \frac{\partial w}{\partial t}=c \frac{\partial u}{\partial y} .
\end{aligned}
$$

Para este caso, utilizamos o esquema de diferenças finitas

$$
\begin{aligned}
\frac{u_{i, j}^{n+1}-u_{i, j}^{n}}{\Delta t} & =c\left(\frac{v_{i+\frac{1}{2}, j}^{n+\frac{1}{2}}-v_{i-\frac{1}{2}, j}^{n+\frac{1}{2}}}{\Delta x}+\frac{w_{i, j+\frac{1}{2}}^{n+\frac{1}{2}}-w_{i, j-\frac{1}{2}}^{n+\frac{1}{2}}}{\Delta y}\right) \\
\frac{v_{i+\frac{1}{2}, j}^{n+\frac{1}{2}}-v_{i+\frac{1}{2}, j}^{n-\frac{1}{2}}}{\Delta t} & =c \frac{u_{i+1, j}^{n}-u_{i, j}^{n}}{\Delta x} \\
\frac{w_{i, j+\frac{1}{2}}^{n+\frac{1}{2}}-w_{i, j+\frac{1}{2}}^{n-\frac{1}{2}}}{\Delta t} & =c \frac{u_{i, j+1}^{n}-u_{i, j}^{n}}{\Delta y} .
\end{aligned}
$$

Novamente, utilizando análise de Fourier e tomando $\left(\begin{array}{c}u^{n} \\ v^{n-\frac{1}{2}} \\ w^{n-\frac{1}{2}}\end{array}\right)=e^{\imath(k x+l y)}\left(\begin{array}{c}\alpha \\ \beta \\ \gamma\end{array}\right)$, obtemos

$$
\left(\begin{array}{ccc}
1 & -\imath a & -\imath b \\
0 & 1 & 0 \\
0 & 0 & 1
\end{array}\right)\left(\begin{array}{c}
u^{n+1} \\
v^{n+\frac{1}{2}} \\
w^{n+\frac{1}{2}}
\end{array}\right)=\left(\begin{array}{ccc}
1 & 0 & 0 \\
\imath a & 1 & 0 \\
\imath b & 0 & 1
\end{array}\right)\left(\begin{array}{c}
u^{n} \\
v^{n-\frac{1}{2}} \\
w^{n-\frac{1}{2}}
\end{array}\right)
$$


onde $a=\frac{2 c \Delta t}{\Delta x} \operatorname{sen} \frac{\theta}{2}, b=\frac{2 c \Delta t}{\Delta y} \operatorname{sen} \frac{\phi}{2}, \theta=k \Delta x$ e $\phi=l \Delta y$. Temos então que

$$
\left(\begin{array}{c}
u^{n+1} \\
v^{n+\frac{1}{2}} \\
w^{n+\frac{1}{2}}
\end{array}\right)=\left(\begin{array}{ccc}
1-a^{2}-b^{2} & \imath a & \imath b \\
\imath a & 1 & 0 \\
\imath b & 0 & 1
\end{array}\right)\left(\begin{array}{c}
u^{n} \\
v^{n-\frac{1}{2}} \\
w^{n-\frac{1}{2}}
\end{array}\right) .
$$

A matriz no segundo membro da equação acima é a matriz de amplificação $G$ do esquema (2.7). Novamente, calculamos os autovalores de $G$ a partir de seu polinômio característico $p(\mu)=$ $(1-\mu)\left[\mu^{2}-\mu\left(2-a^{2}-b^{2}\right)+1\right]$. Tais raízes são $\mu=1 \mathrm{ou}$

$$
\mu=\frac{2-a^{2}-b^{2} \pm \sqrt{\left(a^{2}+b^{2}-2\right)^{2}-4}}{2}
$$

Se $\lambda_{1}=\frac{c \Delta t}{\Delta x}$ e $\lambda_{2}=\frac{c \Delta t}{\Delta y}$ são tais que $\lambda_{1}^{2}+\lambda_{2}^{2} \leq 1$, então $a^{2}+b^{2} \leq 4$. Nesse caso, $2-a^{2}-b^{2} \in$ $[-2,2]$ e, portanto, $\sqrt{\left(a^{2}+b^{2}-2\right)^{2}-4}$ é imaginário puro. Segue que

$$
|\mu|^{2}=\frac{\left(2-a^{2}-b^{2}\right)^{2}}{4}+\frac{4-\left(a^{2}+b^{2}-2\right)^{2}}{4}=1 .
$$

Dessa forma, para $\lambda_{1}^{2}+\lambda_{2}^{2} \leq 1$, o método é estável e convergente de segunda ordem.

Considerações mais gerais acerca de operadores de diferenças finitas e suas propriedades podem ser encontrados em [Str04]. Além da representação discreta das equações diferenciais parciais, formuladas originalmente em domínio contínuo, outra necessidade decorrente do uso de computadores no tratamento numérico de tais equações é a introdução de contornos artificiais, adotadas com o objetivo de delimitar o domínio. O uso desses contornos para simulações de propagação de ondas em domínios ilimitados pode gerar reflexões, de modo que sua escolha deve levar esse fator em consideração. Com esse objetivo, abordamos nas próximas seções condições de contorno propostas por vários autores e, no próximo capítulo, apresentamos algumas aplicações em exemplos diversos.

\subsection{Condições de absorção}

As condições de absorção, também denominadas condições de radiação, consistem em especificações para o comportamento da solução da equação diferencial nos contornos, sem necessidade de ampliação do domínio. Nesta seção, são apresentadas as condições propostas por Engquist e Majda em [EM77] e por Higdon em [Hig87] para a equação da onda acústica.

\subsubsection{Condição de Majda e Engquist}

As condições propostas por Engquist e Majda em [EM77] são baseadas em aproximações paraxiais da equação da onda. O desenvolvimento dessas condições de contorno parte do princípio de que a inserção de um contorno artificial pode gerar ondas que se propagam no sentido oposto ao sentido de propagação da onda original e, então, procura-se atenuar este efeito por meio de uma condição que permita a propagação de ondas em um único sentido. Por esse motivo, as aproximações paraxiais são também conhecidas como One-Way Wave Equations (OWWE), enquanto a equação original é chamada de Two-Way Wave Equation (TWWE). Para o desenvolvimento da condição aqui referida, consideremos, primeiramente, a equação da onda acústica unidimensional

$$
\frac{\partial^{2} u}{\partial t^{2}}=c^{2} \frac{\partial^{2} u}{\partial x^{2}}, c>0
$$

A equação acima é considerada em um domínio ilimitado e, para seu tratamento númerico, analisamos o comportamento de $u$ no domínio $\Omega_{1}=\left[x_{I}, x_{F}\right]$. Tomando soluções do tipo $u(x, t)=$ $e^{\imath(k x-\omega t)}$, temos que a frequência $\omega$ e o número de onda $k$ devem se relacionar por meio da relação de dispersão 


$$
\omega^{2}=c^{2} k^{2} \Rightarrow k= \pm \frac{\omega}{c}
$$

Os números de onda $k>0$ correspondem às ondas que se propagam no sentido positivo de $x$, enquanto os valores $k<0$ representam a propagação no sentido negativo. O objetivo passa a ser a obtenção de uma relação de dispersão de modo a permitir somente ondas com $k>0$, ou seja, preservar apenas os números de onda correspondentes ao sentido no qual a onda originalmente se propagaria em $x=x_{F}$ no domínio ilimitado. Também é importante que a condição para esse contorno resulte em um problema bem-posto quando considerada com a equação da onda. As ondas que se propagam no sentido crescente de $x$ são escritas como $e^{\imath(k x-\omega t)}$ e, no sentido decrescente, escrevem-se como $e^{\imath(-k x-\omega t)}$. A condição que buscamos deve anular as ondas do segundo tipo e, para obtê-la, analisamos a condição $k=-\frac{\omega}{c}$, que corresponde à relação de dispersão da seguinte equação diferencial parcial:

$$
\frac{\partial u}{\partial t}=-c \frac{\partial u}{\partial x} .
$$

Essa condição de contorno apresenta absorção completa das ondas incidentes no contorno considerado e sua obtenção não depende de aproximações, de modo que, para o caso unidimensional, é sugerida apenas uma condição deste tipo. De fato, para ondas se propagando no sentido decrescente de $x$, temos

$$
\frac{\partial u}{\partial t}+c \frac{\partial u}{\partial x}=e^{\imath(-k x-\omega t)}(-i \omega-\imath c k)=e^{\imath(-k x-\omega t)} \imath\left[-\omega-c\left(-\frac{\omega}{c}\right)\right]=0 .
$$

Para o caso bidimensional, consideramos a equação da onda (1.12) com $F \equiv 0$ e realizamos o truncamento do domínio, originalmente ilimitado, analisando a solução em $\Omega_{2}=\left[x_{I}, x_{F}\right] \times\left[y_{I}, y_{F}\right]$. Aqui, desenvolvemos o tratamento para o contorno $x=x_{F}$, e os demais podem ser feitos de modo semelhante. Tomemos soluções de (1.12) do tipo $u=e^{\imath\left(k_{x} x+k_{y} y-\omega t\right)}$. A frequência $\omega$ e os números de onda $k_{x}$ e $k_{y}$ estão relacionados por meio da relação de dispersão

$$
\frac{\omega^{2}}{c^{2}}=k_{x}^{2}+k_{y}^{2}
$$

Da relação acima, temos que $k_{x}= \pm \sqrt{\frac{\omega^{2}}{c^{2}}-k_{y}^{2}}$, ou ainda, $k_{x}= \pm \frac{\omega}{c} \sqrt{1-c^{2} \frac{k_{y}^{2}}{\omega^{2}}}$. De modo análogo ao caso unidimensional, a escolha que fazemos para a relação de dispersão da equação que define a condição de contorno é $k_{x}=-\frac{\omega}{c} \sqrt{1-c^{2} \frac{k_{y}^{2}}{\omega^{2}}}$, que corresponde à seguinte equação diferencial parcial no espaço frequência-número de onda:

$$
\left(\frac{\partial}{\partial x}-\imath \frac{\omega}{c} \sqrt{1-c^{2} \frac{k_{y}^{2}}{\omega^{2}}}\right) u=0 .
$$

Ao contrário do caso unidimensional, para aplicar a condição encontrada em situações práticas, é necessário realizar aproximações para $\sqrt{1-c^{2} \frac{k_{y}^{2}}{\omega^{2}}}$, com $k_{y} \approx 0$. Primeiramente, adotamos a seguinte aproximação para a raiz quadrada, decorrente da consideração feita sobre $k_{y}$, que resulta em $\frac{k_{y}^{2}}{\omega^{2}} \approx 0$ :

$$
\sqrt{1-c^{2} \frac{k_{y}^{2}}{\omega^{2}}}=1+\mathcal{O}\left(c^{2} \frac{k_{y}^{2}}{\omega^{2}}\right)
$$

A transformada de Fourier nos permite observar que a multiplicação por $-\frac{1}{c} \imath \omega$ corresponde, 
nesse caso, à aplicação de $\frac{1}{c} \frac{\partial}{\partial t}$. Levando isso em consideração, obtemos a condição de contorno resultante da primeira aproximação, conhecida como A1:

$$
\left(\frac{\partial}{\partial t}+c \frac{\partial}{\partial x}\right) u=0
$$

Outra possibilidade é a aplicação da primeira aproximação de Taylor ou Padé $\sqrt{1-c^{2} \frac{k_{y}^{2}}{\omega^{2}}}=$ $1-c^{2} \frac{1}{2} \frac{k_{y}^{2}}{\omega^{2}}+\mathcal{O}\left(c^{4} \frac{k_{y}^{4}}{\omega^{4}}\right)$. A aplicação dessa aproximação a (2.9) resulta em

$$
\begin{aligned}
& {\left[\frac{\partial}{\partial x}-\imath \frac{\omega}{c}\left(1-c^{2} \frac{1}{2} \frac{k_{y}^{2}}{\omega^{2}}\right)\right] u=0 \Rightarrow\left[\frac{\partial}{\partial x}-\imath \frac{\omega}{c}+\imath c \frac{1}{2} \frac{k_{y}^{2}}{\omega}\right]=0 } \\
\Rightarrow & \left(-\frac{\partial}{\partial x} \cdot \imath \omega+\frac{(-\imath \omega)^{2}}{c}-\imath^{2} c \frac{1}{2} k_{y}^{2}\right) u=0 .
\end{aligned}
$$

Substituindo novamente $-\imath \omega$ por $\frac{\partial}{\partial t}$ e $-\imath k_{y}$ por $\frac{\partial}{\partial y}$, obtemos a condição de contorno A2:

$$
\left(\frac{\partial^{2}}{\partial t^{2}}+c \frac{\partial^{2}}{\partial x \partial t}-c^{2} \frac{1}{2} \frac{\partial^{2}}{\partial y^{2}}\right) u=0 .
$$

É possível ainda obter aproximações de ordem mais alta, desde que se observe que a condição de contorno deve gerar um problema bem-posto. Um estudo mais detalhado, apresentado em [EM77], mostra que a condição de contorno decorrente da segunda aproximação de Taylor para a raiz quadrada resulta em um problema mal-posto, enquanto a aproximação de Padé se mostra adequada neste sentido. Também de acordo com o trabalho de Engquist e Majda, as condições apresentadas anteriormente geram problemas bem-postos.

Discretização: Para a discretização das condições dadas, Engquist e Majda sugerem em [EM77] o seguinte operador para a condição (2.10) no contorno $x_{N}=x_{F}$ :

$$
c\left(\frac{u_{N, j}^{n}-u_{N-1, j}^{n}}{\Delta x}+\frac{u_{N, j}^{n+1}-u_{N-1, j}^{n+1}}{\Delta x}\right)+\left(\frac{u_{N, j}^{n+1}-u_{N, j}^{n}}{\Delta t}+\frac{u_{N-1, j}^{n+1}-u_{N-1, j}^{n}}{\Delta t}\right)=0 .
$$

Já para a condição A2, temos

$$
\begin{array}{r}
\frac{u_{N, j}^{n+1}-u_{N, j}^{n-1}-u_{N-1, j}^{n+1}+u_{N-1, j}^{n-1}}{\Delta t \Delta x}+\frac{c}{2}\left(\frac{u_{N, j}^{n+1}-2 u_{N, j}^{n}+u_{N, j}^{n+1}+u_{N-1, j}^{n+1}-2 u_{N, j}^{n}+u_{N, j}^{n+1}}{\Delta t^{2}}\right) \\
-\frac{c^{2}}{4}\left(\frac{u_{N, j+1}^{n-1}-2 u_{N, j}^{n-1}+u_{N, j-1}^{n-1}+u_{N-1, j+1}^{n+1}-2 u_{N-1, j}^{n+1}+u_{N-1, j-1}^{n+1}}{\Delta y^{2}}\right)=0
\end{array}
$$

Outras considerações sobre o processo de discretização das condições de contorno podem ser encontradas no mesmo trabalho. Um estudo mais detalhado, apresentado em [EM79], mostra que o tratamento nos cantos do modelo pode ser realizado por meio da aplicação, ao ponto do canto e aos pontos do contorno mais próximos a este, uma condição que envolve apenas derivadas de primeira ordem. Para o canto $\left(x_{F}, y_{F}\right)$, por exemplo, é sugerida a condição: 


$$
\left[\sqrt{2} \frac{\partial}{\partial t}+c\left(\frac{\partial}{\partial x}+\frac{\partial}{\partial y}\right)\right] u=0
$$

com a discretização

$$
\begin{aligned}
\frac{u_{i, j}^{n}-u_{i-1, j}^{n}+u_{i, j}^{n+1}-u_{i-1, j}^{n+1}}{\Delta x}+\frac{u_{i, j}^{n}-u_{i, j-1}^{n}+u_{i, j}^{n+1}-u_{i, j-1}^{n+1}}{\Delta y} & + \\
c \frac{2}{3} \sqrt{2}\left(\frac{u_{i, j-1}^{n+1}-u_{i, j-1}^{n}+u_{j-1, j}^{n+1}-u_{j-1, j}^{n}+u_{i, j}^{n+1}-u_{i, j}^{n}}{\Delta t}\right) & =0 .
\end{aligned}
$$

Podemos verificar a capacidade de absorção da condição (2.9). Consideramos $u$ como a superposição de duas ondas

$$
u=u^{+}+R u^{-},
$$

onde $u^{+}=e^{\imath\left(k_{x} x+k_{y} y-\omega t\right)}, u^{-}=e^{\imath\left(-k_{x} x+k_{y} y-\omega t\right)}$ e $R$ é um coeficiente de reflexão. O vetor de número de onda é dado por $k=\left(\begin{array}{c}\cos \phi \\ \operatorname{sen} \phi\end{array}\right)$, com $\phi$ sendo o ângulo de incidência entre o eixo $x$ e o vetor $k$. Aplicando o operador referente à condição (2.9) à função (2.14), temos

$$
\begin{aligned}
\left(\frac{\partial}{\partial x}+\imath \frac{\omega}{c} \sqrt{1-c^{2} \frac{k_{y}^{2}}{\omega^{2}}}\right)\left(u^{+}+R u^{-}\right) & =\left(\frac{\partial}{\partial x}+\imath \frac{\omega}{c} \sqrt{1-c^{2} \frac{k_{y}^{2}}{\omega^{2}}}\right) u^{+} \\
& +R\left(\frac{\partial}{\partial x}+\imath \frac{\omega}{c} \sqrt{1-c^{2} \frac{k_{y}^{2}}{\omega^{2}}}\right) u^{-}=0 .
\end{aligned}
$$

Utilizando as definições de $u^{+}$e $u^{-}$, temos

$$
\begin{aligned}
\Rightarrow\left(\frac{\partial}{\partial x}+\imath \frac{\omega}{c} \sqrt{1-c^{2} \frac{k_{y}^{2}}{\omega^{2}}}\right)\left(u^{+}+R u^{-}\right) & =e^{\imath\left(k_{x} x+k_{y} y-\omega t\right)}\left(\imath k_{x}+\imath \sqrt{\frac{\omega^{2}}{c^{2}}-k_{y}^{2}}\right) \\
& +\operatorname{Re}^{\imath\left(-k_{x} x+k_{y} y-\omega t\right)}\left(-\imath k_{x}+\imath \sqrt{\frac{\omega^{2}}{c^{2}}-k_{y}^{2}}\right)=0 .
\end{aligned}
$$

Com a equação acima, podemos encontrar o valor de $R$ :

$$
R=-e^{2 \imath k_{x} x} \frac{\sqrt{\frac{\omega^{2}}{c^{2}}-k_{y}^{2}}+k_{x}}{\sqrt{\frac{\omega^{2}}{c^{2}}-k_{y}^{2}}-k_{x}}=-e^{2 \imath k_{x} x} \frac{\frac{\omega^{2}}{c^{2}}-k_{y}^{2}-k_{x}^{2}}{\left(\sqrt{\frac{\omega^{2}}{c^{2}}-k_{y}^{2}}-k_{x}\right)^{2}}=0,
$$

pois $\frac{\omega^{2}}{c^{2}}=k_{x}^{2}+k_{y}^{2}$. Isso significa que a condição (2.9) absorve perfeitamente ondas que se deslocam para oeste, denotadas por $u^{-}$.

Para a condição $(2.10)$, quando $\omega=1$, observamos que $k_{x}=\frac{\cos \phi}{c}$ e $k_{y}=\frac{\operatorname{sen} \phi}{c}$. Essa consideração nos permite obter um valor para $|R|$ :

$$
\begin{gathered}
\left(\frac{\partial}{\partial t}+c \frac{\partial}{\partial x}\right)\left(u^{+}+R u^{-}\right)=e^{\imath\left(k_{x} x+k_{y} y-\omega t\right)}\left(-\imath \omega+\imath c k_{x}\right)+R e^{\imath\left(-k_{x} x+k_{y} y-\omega t\right)}\left(-\imath \omega-\imath c k_{x}\right)=0 \\
\Rightarrow|R|=\left|e^{2 \imath k_{x} x}\right|\left|\frac{\cos \phi-\omega}{\cos \phi+\omega}\right|=\left|\frac{\cos \phi-1}{\cos \phi+1}\right| .
\end{gathered}
$$


O mesmo procedimento aplicado à condição (2.11) resulta em

$$
\begin{aligned}
&\left(\frac{\partial^{2}}{\partial t^{2}}+c \frac{\partial^{2}}{\partial x \partial t}-c^{2} \frac{1}{2} \frac{\partial^{2}}{\partial y^{2}}\right)\left(u^{+}+R u^{-}\right)=0 \\
& \Rightarrow|R|=\left|e^{2 \imath k_{x} x}\right|\left|\frac{\omega^{2}-\omega \cos \phi-\frac{1}{2} \operatorname{sen}^{2} \phi}{\omega^{2}+\omega \cos \phi-\frac{1}{2} \operatorname{sen}^{2} \phi}\right| \\
& \Rightarrow|R|=\left|\frac{1-\cos \phi-\frac{1}{2} \operatorname{sen}^{2} \phi}{1+\cos \phi-\frac{1}{2} \operatorname{sen}^{2} \phi}\right| \\
& \Rightarrow|R|=\left|\frac{\frac{1}{2}+\frac{\cos ^{2} \phi}{2}-\cos \phi}{\frac{1}{2}+\frac{\cos ^{2} \phi}{2}+\cos \phi}\right|=\left|\frac{\cos \phi-1}{\cos \phi+1}\right|^{2}
\end{aligned}
$$

A análise acima sugere que o método proposto funcionará bem quando $\phi=0$, ou seja, quando as ondas se propagam na direção normal do contorno. Para casos mais gerais, podemos considerar as condições propostas por Higdon, abordadas na próxima seção.

\subsubsection{Condição de Higdon}

Em seus trabalhos [Hig87], [Hig90] e [Hig91], Robert L. Higdon desenvolveu uma classe de condições de contorno supondo uma onda se propagando para fora do domínio com um ângulo de incidência $\alpha$ e velocidade $c$. Em duas dimensões, essa onda é escrita como

$$
u=f(x \cos \alpha+y \operatorname{sen} \alpha+c t)
$$

e o sentido de propagação nesse caso depende de $\alpha$, o que possibilita realizar uma abordagem que seja válida independentemente do contorno considerado. A obtenção da condição proposta por Higdon leva em consideração o operador

$$
L=\cos \alpha \frac{\partial}{\partial t}-c \frac{\partial}{\partial x} .
$$

Aplicando-o a (2.15), obtemos

$$
L u=c \cos \alpha \cdot f^{\prime}(x \cos \alpha+y \operatorname{sen} \alpha+c t)-c \cos \alpha \cdot f^{\prime}(x \cos \alpha+y \operatorname{sen} \alpha+c t)=0 .
$$

Isso significa que ondas da forma (2.15) se anulam ao atingir o contorno no qual é definida essa condição. A forma geral da condição de contorno de Higdon é a seguinte:

$$
\left[\prod_{j=1}^{p}\left(\cos \alpha_{k} \frac{\partial}{\partial t}-c \frac{\partial}{\partial x}\right)\right] u=0, \quad\left|\alpha_{k}\right|<\frac{\pi}{2} .
$$

Discretização: Em seu trabalho [Hig90], Higdon sugere que a discretização dos operadores de (2.16) seja feita por meio de aproximações do tipo

$$
\left(\cos \alpha_{k} \frac{\partial}{\partial t}-c \frac{\partial}{\partial x}\right) \approx \cos \alpha_{k}\left(\frac{I-S_{t}^{-}}{\Delta t}\right)\left[(1-a) I+a S_{x}\right]-c\left(\frac{S_{x}-I}{\Delta x}\right)\left[(1-b) I+b S_{t}^{-}\right],
$$

onde $S_{x} u_{i}=u_{i-1}$ ou $S_{x} u_{i}=u_{i+1}$, tomado para cada contorno de modo a utilizar pontos do interior do domínio, e $S_{t}^{-} u^{n}=u^{n-1}$.

A condição (2.16) é satisfeita por qualquer combinação linear de ondas planas se propagando para fora do domínio com ângulos de incidência $\pm \alpha_{k}, k=1, \ldots, p$ com velocidade $c$, de modo que não ocorre reflexão para tais ondas.

É possível utilizar os parâmetros $\alpha_{k}$ para otimizar a condição de contorno, aumentando sua 
capacidade de absorção e tornando-a mais eficiente, se houver informações prévias sobre o ângulo de incidência da onda no contorno. Além disso, esse método se destaca por não ser instável para valores altos de $\frac{v_{p}}{v_{s}}$, no caso das ondas elásticas, e pode ser estendido ao caso tridimensional a partir do raciocínio aplicado ao bidimensional.

É importante observar que a condição de primeira ordem para a equação bidimensional proposta por Engquist e Majda abordada anteriormente corresponde à de Higdon com $p=1$ e $\alpha_{1}=0$, bem como a condição para a equação unidimensional.

Para o estudo das propriedades de reflexão da condição de Higdon, consideremos novamente uma solução da forma (2.14), com o vetor $\mathbf{k}=\frac{\omega}{c}\left(\begin{array}{c}\cos \phi \\ \operatorname{sen} \phi\end{array}\right)$, onde $\phi$ é o ângulo entre $\mathbf{k}$ e o eixo $x$. Aplicando a condição de contorno (2.16) a essa solução, temos

$$
\begin{aligned}
{\left[\prod_{j=1}^{p}\left(\cos \alpha_{k} \frac{\partial}{\partial t}-c \frac{\partial}{\partial x}\right)\right] u } & =\left[\prod_{j=1}^{p}\left(\cos \alpha_{k} \frac{\partial}{\partial t}-c \frac{\partial}{\partial x}\right)\right]\left(u^{+}+R u^{-}\right) \\
\Rightarrow\left[\prod_{j=1}^{p}\left(\cos \alpha_{k} \frac{\partial}{\partial t}-c \frac{\partial}{\partial x}\right)\right] u & =\prod_{j=1}^{p}\left[e^{\imath\left(k_{x} x+k_{y} y-\omega t\right)}\left(\omega \cos \alpha_{k}-c k_{x}\right)\right] \\
& +R \prod_{j=1}^{p}\left[e^{\imath\left(-k_{x} x+k_{y} y-\omega t\right)}\left(\omega \cos \alpha_{k}+c k_{x}\right)\right] \\
\Rightarrow\left[\prod_{j=1}^{p}\left(\cos \alpha_{k} \frac{\partial}{\partial t}-c \frac{\partial}{\partial x}\right)\right] u & =\prod_{j=1}^{p}\left[e^{\imath\left(k_{x} x+k_{y} y-\omega t\right)}\left(\omega \cos \alpha_{k}-\omega \cos \phi\right)\right] \\
& +R \prod_{j=1}^{p}\left[e^{\imath\left(-k_{x} x+k_{y} y-\omega t\right)}\left(\omega \cos \alpha_{k}+\omega \cos \phi\right)\right] \\
\Rightarrow R= & \prod_{j=1}^{p}\left(\frac{\cos \alpha_{k}-\cos \phi}{\cos \alpha_{k}+\cos \phi}\right)
\end{aligned}
$$

Aqui, os fatores do tipo $e^{z}$ foram omitidos por não influenciarem no valor absoluto de $R$. Como vemos, para $p=1,2$, se $\alpha_{k}=0, k=1, \ldots, p$, então os coeficientes de reflexão obtidos são iguais aos referentes às condições de Engquist e Majda.

\subsection{Camadas de absorção}

O uso de camadas de absorção consiste, em linhas gerais, em expandir o domínio no qual se deseja calcular a solução da equação e, ao longo da região acrescentada, amortecer as ondas de modo a reduzir sua reflexão de volta ao domínio original. Dessa forma, é possível a aplicação das condições de Dirichlet e Neumann, que não possuem efeito de amortecimento ou absorção das ondas incidentes, sem prejuízos para a simulação do fenômeno desejado. Neste trabalho, abordamos quatro propostas de condições desse tipo: a condição de Cerjan, a de Sochacki, duas formas para a Perfectly Matched Layer (PML) e duas propostas de Hybrid Absorbing Boundary Conditions (HABCs).

As condições de Cerjan, Sochacki e PML não estabelecem condições especificamente para os contornos do domínio, de modo que é necessária a aplicação de condições de contorno

$$
\begin{aligned}
& B_{1} u\left(x, y_{I}-L_{y}, t\right)=0 \\
& B_{2} u\left(x, y_{F}+L_{y}, t\right)=0 \\
& B_{3} u\left(x_{I}-L_{x}, y, t\right)=0 \\
& B_{4} u\left(x_{F}+L_{x}, y, t\right)=0,
\end{aligned}
$$

para a equação da onda acústica. As condições de contorno $B_{1}, B_{2}, B_{3}$ e $B_{4}$ podem ser, conforme 
a sugestão de Sochacki et al., as condições de Dirichlet, Neumann ou uma condição de absorção. Já as HABCs incluem condições para o contorno do domínio, como veremos a seguir.

\subsubsection{Condição de Cerjan}

A condição proposta por Cerjan é baseada na redução gradativa da amplitude das ondas ao longo de uma região de amortecimento em torno do domínio original. Essa condição possui a vantagem de ter sua efetividade mantida com o aumento do ângulo de incidência da onda, além de ser consideravelmente simples por não demandar alterações na equação e poder ser aplicada diretamente na equação discretizada. O desenvolvimento apresentado a seguir está de acordo com o trabalho de Cerjan et al. [CKKR85].

Para aplicar o método de Cerjan, realizamos uma expansão do domínio $\Omega_{2}=\left[x_{I}, x_{F}\right] \times\left[y_{I}, y_{F}\right]$ acrescentando faixas de largura $L_{x}$ e $L_{y}$ em torno do mesmo e, desse modo, passamos a considerar o problema no domínio $\left[x_{I}-L_{x}, x_{F}+L_{x}\right] \times\left[y_{I}-L_{y}, y_{F}+L_{y}\right]$.

A aplicação da condição de contorno proposta por Cerjan consiste em calcular a solução no domínio original de acordo com o método de discretização escolhido (no nosso caso, utilizamos a discretização padrão para a equação na forma de segunda ordem (2.3)) e, para $x \in\left[x_{I}-L_{x}, x_{I}\right]$, $x \in\left[x_{F}, x_{F}+L_{x}\right], y \in\left[y_{I}-L_{y}, y_{I}\right]$ ou $y \in\left[y_{F}, y_{F}+L_{y}\right]$, calcular a solução da seguinte forma:

$$
u_{i, j}^{n+1}=G\left(x_{i}, y_{j}\right)\left[2 u_{i, j}^{n}-u_{i, j}^{n-1}+\Delta t^{2} c^{2}\left(\frac{u_{i+1, j}^{n}-2 u_{i, j}^{n}+u_{i-1, j}^{n}}{\Delta x^{2}}+\frac{u_{i, j-1}^{n}-2 u_{i, j}^{n}+u_{i, j-1}^{n}}{\Delta y^{2}}\right)\right] .
$$

com $x_{i}=x_{I}-L_{x}+i \Delta x$ ou $x_{i}=x_{F}+i \Delta x, y_{j}=y_{I}-L_{y}+j \Delta y$ ou $y_{j}=y_{F}+j \Delta y$ e $G(x, y)$ sendo uma função de amortecimento, responsável por reduzir a amplitude das ondas que se propagam na região acrescentada ao domínio. Aqui, o termo $F(x, y, t)$ é omitido por considerarmos que o mesmo seja nulo fora do domínio de interesse. Um exemplo de função apresentado por Cerjan et al. para amortecimento na direção $x$ é $G\left(x_{i}, y_{j}\right)=e^{-[0.015(20-i)]^{2}}$, para $1 \leq i \leq 20$, considerandose que a região acrescentada possui 20 pontos da grade na direção $x$. De modo mais geral, o amortecimento da solução nessa condição de contorno pode ser realizado por meio de uma função do tipo $G(i)=e^{-\tilde{\lambda}^{2}\left(i_{0}-i\right)^{2}}, i=0, \ldots, i_{0}$, com $\tilde{\lambda}$ sendo um coeficiente de atenuação, $i$ o índice do ponto considerado na malha e $i_{0}$ o índice do ponto em que se inicia a região de amortecimento na direção considerada.

Este tipo de condição de contorno não leva em consideração a estrutura da equação, afetando diretamente a resolução numérica, o que torna simples a sua aplicação, independentemente da forma da equação. Além disso, não demanda considerações particulares em relação à discretização, ou seja, pode-se adotar os mesmos operadores aplicados à resolução da equação original. No entanto, uma possível dificuldade em relação ao uso dessa condição de contorno é o caráter empírico do fator de amortecimento, cuja escolha afeta diretamente a eficácia do método.

\subsubsection{Condição de Sochacki}

A condição de contorno proposta por Sochacki tem em vista o uso dos fundamentos matemáticos por trás das equações a serem resolvidas numericamente; trata-se, portanto, de uma abordagem diferente da adotada por Cerjan em seu método. O objetivo é o desenvolvimento de uma condição de contorno que possa ser aplicada de forma independente do processo de discretização, por meio de alterações na estrutura da equação. Esse método também pode ser utilizado em equações parabólicas, conforme indicado no trabalho de Sochacki et al. [SKG $\left.{ }^{+} 87\right]$, e seu desenvolvimento tem como base o uso de um efeito de absorção de choque para gerar o decaimento da onda em uma região em torno do modelo.

Consideremos, a princípio, a equação da onda em um domínio restrito, como fizemos na seção anterior, e expandimos o domínio da mesma forma. O método proposto por Sochacki consiste em reformular a equação da onda, inserindo um termo cuja função será gerar atenuação das ondas 
no decorrer de sua propagação ao longo da faixa acrescentada. O termo inserido será da forma $2 A(x, y) \frac{\partial u}{\partial t}$, com $A(x, y)=0$ para $(x, y) \in \Omega_{2}$. Assim, a equação que trataremos numericamente será a seguinte:

$$
\frac{\partial^{2} u}{\partial t^{2}}+2 A(x, y) \frac{\partial u}{\partial t}=c^{2}\left(\frac{\partial^{2} u}{\partial x^{2}}+\frac{\partial^{2} u}{\partial y^{2}}\right)+F(x, y, t) .
$$

Discretização: A discretização da equação anterior pode ser feita com os operadores convencionais, levando em consideração as ordens das derivadas que se deseja aproximar. Para os termos de $2^{\mathrm{a}}$ ordem no tempo, realizamos a discretização conforme (2.3), enquanto para o termo de amortecimento, que é de $1^{\mathrm{a}}$ ordem no tempo, utilizamos um operador de diferenças centradas. Aplicando os operadores em $u_{i, j}^{n}$, é possível encontrar uma expressão para $u_{i, j}^{n+1}$ dependente da solução nos níveis $n$ e $n-1$. Por exemplo, para a condição (2.18) associada à equação (1.12), obtemos

$$
\begin{aligned}
u_{i, j}^{n+1}= & \left(\frac{\Delta t^{2}}{1+\Delta t A\left(x_{i}, y_{j}\right)}\right)\left[\frac{2 u_{i, j}^{n}}{\Delta t^{2}}-\left(\frac{1-\Delta t A\left(x_{i}, y_{j}\right)}{\Delta t^{2}}\right) u_{i, j}^{n-1}\right]+ \\
& \left(\frac{\Delta t^{2}}{1+\Delta t A\left(x_{i}, y_{j}\right)}\right)\left[c^{2} \frac{u_{i+1, j}^{n}-2 u_{i, j}^{n}+u_{i-1, j}^{n}}{\Delta x^{2}}+c^{2} \frac{u_{i, j+1}^{n}-2 u_{i, j}^{n}+u_{i, j-1}^{n}}{\Delta y^{2}}+F_{i, j}^{n}\right] .
\end{aligned}
$$

É importante observar que essa condição de contorno também é aplicável a problemas com coeficientes variáveis e pode-se ainda utilizar o método somente nos cantos do modelo, aplicando nas faixas ao longo das bordas outras condições de contorno.

As propriedades da função $A(x, y)$ estão relacionadas ao objetivo da condição de contorno, ou seja, à capacidade de amortecimento das ondas incidentes. Para a equação da onda acústica, por exemplo, buscamos soluções da forma

$$
u(x, y, t)=e^{-A(x, y) t} u_{1}
$$

onde $u_{1}$ é uma solução do problema sem o termo de amortecimento. Podemos então concluir que $A(x, y)$ deve ser uma função positiva. Se $A(x, y)$ for limitada, duas vezes continuamente diferenciável e se suas derivadas em relação a $x$ e $y$ forem suficientemente pequenas em quaisquer pontos (de modo que as soluções obtidas sejam próximas de (2.19), mesmo que $A(x, y)$ não seja constante), então as ondas decaem suficientemente nos contornos.

No trabalho de Sochacki et al., são sugeridas algumas formas para $A(x, y)$. A seguinte forma é denominada Amortecedor linear:

$$
\begin{array}{ll}
A(x, y)=\alpha\left(x-\frac{x_{1}+x_{F}}{2}\right)\left(y-\frac{y_{1}+y_{F}}{2}\right) & \text { (canto superior direito) } \\
A(x, y)=\alpha\left(\frac{x_{1}+x_{I}}{2}-x\right)\left(y-\frac{y_{1}+y_{F}}{2}\right) & \text { (canto superior esquerdo) } \\
A(x, y)=\alpha\left(x-\frac{x_{1}+x_{F}}{2}\right)\left(\frac{y_{1}+y_{I}}{2}-y\right) & \text { (canto inferior direito) } \\
A(x, y)=\alpha\left(\frac{x_{1}+x_{I}}{2}-x\right)\left(\frac{y_{1}+y_{I}}{2}-y\right) & \text { (canto inferior esquerdo) }
\end{array}
$$




$$
\begin{array}{ll}
A(x, y)=\alpha\left(y-\frac{y_{1}+y_{F}}{2}\right) & \text { (borda superior) } \\
A(x, y)=\alpha\left(\frac{y_{1}+y_{I}}{2}-y\right) & \text { (borda inferior) } \\
A(x, y)=\alpha\left(x-\frac{x_{1}+x_{F}}{2}\right) & \text { (borda direita) } \\
A(x, y)=\alpha\left(\frac{x_{1}+x_{I}}{2}-x\right) & \text { (borda esquerda) }
\end{array}
$$

$\operatorname{com} S=\|A\|_{\infty}, \alpha=\frac{S}{L_{x} L_{y}}, L_{x}$ e $L_{y}$ sendo a espessura das camadas acrescentadas ao domínio nas direções $x$ e $y$, respectivamente, $x_{1}=x_{I}-\Delta x$ (para os cantos ou a borda esquerdos), $x_{1}=x_{F}+\Delta x$ (cantos ou borda direitos), $y_{1}=y_{I}-\Delta y$ (cantos e borda inferiores), $y_{1}=y_{F}+\Delta y$ (cantos e borda superiores). Para o caso unidimensional, também é acrescentado o termo $2 A(x) \frac{\partial u}{\partial t}$ à equação a ser resolvida, e o tratamento é realizado conforme as formulações apresentadas anteriormente para as bordas do domínio bidimensional.

São sugeridos outros 4 tipos de funções $A(x, y)$ que podem ser utilizadas conforme a formulação apresentada para o amortecedor linear. Para o canto superior direito, por exemplo, tais funções são formuladas como segue:

Amortecedor expoente: $A(x, y)=\alpha\left[\left(x-x_{F}\right)\left(y-y_{F}\right)\right]^{\beta}, \beta=\frac{\ln (S)}{\ln \left(L_{x} L_{y}\right)-\ln (\Delta x \Delta y)}$, $\alpha=(\Delta x \Delta y)^{-\beta}$

Amortecedor cúbico: $A(x, y)=\alpha\left(x-x_{F}\right)^{3}\left(y-y_{F}\right)^{3}, \alpha=\frac{S}{L_{x}^{3} L_{y}^{3}}$;

Amortecedor exponencial: $A(x, y)=\alpha e^{\beta\left(x-x_{F}\right)\left(y-y_{F}\right)}, \beta=\frac{\ln (S)}{\left(L_{x}-\Delta x\right)\left(L_{y}-\Delta y\right)}$, $\alpha=e^{-\beta \Delta x \Delta y} ;$

Amortecedor Gaussiano: $A(x, y)=\alpha e^{\frac{-\beta}{\left(x-x_{F}\right)+\left(y-y_{F}\right)}}, \beta=\frac{(\Delta x+\Delta y)\left(L_{x}+L_{y}\right)}{\left(L_{x}+L_{y}\right)-(\Delta x+\Delta y)} \ln (S)$, $\alpha=e^{\frac{\beta}{\Delta x+\Delta y}}$.

No caso do amortecedor exponencial, podemos observar que, embora não assuma valor nulo em nenhum ponto, é possível obter $A(x, y)$ suficientemente pequeno com a escolha adequada de $\alpha$. Outra observação importante é a de que, no caso do amortecedor linear, $A(x, y)$ e suas derivadas não se anulam nas bordas de $\Omega_{2}$. Apesar disso, a efetividade desse amortecedor pode ser observada em situações práticas. O amortecedor Gaussiano, por sua vez, não assume valor nulo, mas é tal que $\lim _{(x, y) \rightarrow\left(x^{*}, y^{*}\right)} A(x, y)=0, \operatorname{com}\left(x^{*}, y^{*}\right)$ nas bordas do domínio $\Omega_{2}$. Essas observações indicam que, embora as propriedades mencionadas anteriormente possam ser consideradas de modo a assegurar a eficácia do método, é possível obter resultados satisfatórios sem que as mesmas sejam verificadas. Assim como a condição apresentada por Cerjan, o método de Sochacki também depende da escolha de parâmetros que afetam diretamente seu desempenho.

\subsubsection{Perfectly Matched Layer (PML)}

A Perfectly Matched Layer (PML) é uma condição de contorno que, assim como as condições de Cerjan e Sochacki, tem como base a extensão do domínio e a modificação da equação na região acrescentada, de modo a gerar o decaimento da solução e reduzir a reflexão das ondas propagadas. O desenvolvimento aqui apresentado tem como base os trabalhos de Johnson [Joh08], Komatitsch e Tromp [KT03], Fichtner [Fic10] e Grote [GS10]. Para ambas as abordagens apresentadas a seguir, são utilizadas funções de amortecimento $d_{x}(x)$ e $d_{y}(y)$, dependentes da localização dos pontos na 
camada de amortecimento, que são frequentemente tomadas como funções quadráticas ou cúbicas da menor distância entre o ponto considerado e o domínio no qual a solução é observada. Para o interior do domínio de interesse, tais funções são identicamente nulas.

\section{PML para o sistema de primeira ordem na variável temporal}

Consideremos a equação (1.12), definida em um domínio limitado de modo que $F$ tenha suporte contido neste domínio. Assim, fora do domínio, podemos considerar $F=0$. Para formular a PML, escrevemos (1.12) como segue:

$$
\nabla \cdot(c \nabla u)=\frac{1}{c} \frac{\partial^{2} u}{\partial t^{2}}
$$

ou ainda, em forma de sistema:

$$
\left\{\begin{array}{l}
\frac{\partial u}{\partial t}=c \nabla \cdot v \\
\frac{\partial v}{\partial t}=c \nabla u
\end{array}\right.
$$

O procedimento que realizamos a seguir é chamado de complex coordinate stretching. Primeiramente, realizamos uma extensão analítica da solução a um contorno de $x$ complexo, de modo que ondas oscilantes se tornam ondas de decaimento exponencial fora do domínio limitado sem reflexões. Ainda no espaço infinito, é realizada uma mudança de coordenadas para expressar a coordenada $x$ complexa em função da coordenada real. Neste espaço, as soluções devem assumir a forma

$$
w(\mathbf{x}, t)=\sum_{\mathbf{k}, \omega} W_{\mathbf{k}, \omega} e^{\imath(\mathbf{k} \cdot \mathbf{x}-\omega t)}
$$

Cada componente $W_{\mathbf{k}, \omega} e^{\imath(\mathbf{k} \cdot \mathbf{x}-\omega t)}$ é uma função analítica, de modo que podemos realizar extensões analíticas destas e assim, calcular a solução para valores complexos de $x$. Podemos então observar o que ocorre com a solução quando, para $x$ tal que $R e x \geq \tilde{k}_{x}$, com $\tilde{k}_{x}$ constante, adicionamos uma parte imaginária linearmente crescente. Nesse caso, como $e^{\imath k_{x}(\operatorname{Re} x+\imath \operatorname{Im} x)}=e^{\imath k_{x} \operatorname{Re} x} e^{-k_{x} \operatorname{Im} x}$, a solução é exponencialmente descrescente devido ao fator $e^{-k_{x} \operatorname{Im} x}$, supondo $k_{x}>0$, ou seja, ondas se deslocando no sentido de $x>0$.

Seja $\tilde{x}$ a coordenada complexa e $x$ sua parte real. Assim, $\tilde{x}=x+\imath g(x)$ determina o comportamento da coordenada complexa. Queremos que a equação (2.20) seja válida para a coordenada $\tilde{x}$ e, dessa forma, escrevemos o sistema (2.21) substituindo a diferenciação em relação à coordenada $x$ pela diferenciação em relação à coordenada $\tilde{x}$. Para retornar para a variável real, é suficiente observar que $\partial_{\tilde{x}}=\left(1+\imath \frac{d g}{d x}\right) \partial x$. Logo, o processo de aplicação da PML pode ser sintetizado na mudança

$$
\frac{\partial}{\partial x} \rightarrow \frac{1}{1+\imath \frac{d_{x}(x)}{\omega}} \frac{\partial}{\partial x}
$$

onde $\frac{d_{x}}{\omega}=\frac{d f}{d x}$.

Aplicando a transformação (2.22) à equação cuja solução se deseja simular, obtemos a equação que deve ser resolvida em uma camada acrescentada em torno do domínio de interesse, na qual a onda decairá de modo a não existir reflexão causada pelo truncamento do domínio infinito. A princípio, poderíamos tomar $d_{x}$ tão grande quanto fosse necessário para atingir o amortecimento desejado e, dessa forma, seria possível utilizar uma camada mais fina. No entanto, esse procedimento pode gerar reflexões numéricas causadas pelo processo de discretização. Assim, é usual tomar $d_{x}(x)$ como uma função quadrática ou cúbica partindo de 0 .

Para as ondas se propagando no sentido $x<0$, para as quais $k_{x}<0$, podemos também aplicar a transformação (2.22). Nesse caso, as soluções $e^{\imath k_{x} x} e^{-\frac{k_{x}}{\omega}\left(-\int_{x}^{0} d_{x}\left(x^{\prime}\right) d x^{\prime}\right)}$ decaem quando $x$ decresce, uma vez que $d_{x}>0, \forall x \in \mathbb{R}$ e 


$$
-\frac{k_{x}}{\omega}\left(-\int_{x}^{0} d_{x}\left(x^{\prime}\right) d x^{\prime}\right)=\frac{k_{x}}{\omega} \int_{x}^{0} d_{x}\left(x^{\prime}\right) d x^{\prime}<0 .
$$

Para a dimensão $y$, o procedimento é análogo e nas regiões em que deve ser realizado amortecimento em ambas as dimensões, como nos cantos do modelo, a equação pode ser transformada nas duas variáveis. A transformação (2.22) resulta em uma equação cuja solução tem decaimento exponencial no domínio da frequência. Para utilizar a PML no domínio do tempo, devemos realizar uma transformação para a variável temporal. Consideremos, primeiramente, o sistema referente à equação da onda unidimensional:

$$
\left\{\begin{array}{l}
\frac{\partial u}{\partial t}=c \frac{\partial v}{\partial x}=-\imath \omega u \\
\frac{\partial v}{\partial t}=c \frac{\partial u}{\partial x}=-\imath \omega v
\end{array}\right.
$$

Aplicando a substituição (2.22) ao sistema acima e multiplicando ambos os membros por $1+\frac{d_{x}}{\omega}$, obtemos o seguinte sistema para a PML, que pode ser transformado para o domínio do tempo diretamente, sem considerações adicionais:

$$
\left\{\begin{array} { l } 
{ c \frac { \partial v } { \partial x } = - \imath \omega u + d _ { x } u } \\
{ c \frac { \partial u } { \partial x } = - \imath \omega v + d _ { x } v }
\end{array} \Rightarrow \left\{\begin{array}{l}
\frac{\partial u}{\partial t}=c \frac{\partial v}{\partial x}-d_{x} u \\
\frac{\partial v}{\partial t}=c \frac{\partial u}{\partial x}-d_{x} v
\end{array} .\right.\right.
$$

Para a equação bidimensional, o sistema (2.21) assume a forma

$$
\left\{\begin{array}{l}
\frac{\partial u}{\partial t}=c \frac{\partial v}{\partial x}+c \frac{\partial w}{\partial y}=-i \omega u \\
\frac{\partial v}{\partial t}=c \frac{\partial u}{\partial x} \\
\frac{\partial w}{\partial t}=c \frac{\partial u}{\partial y}
\end{array} .\right.
$$

Aplicando a transformação (2.22) às duas primeiras equações do sistema acima e multiplicando por $1+d_{x} \frac{\imath}{\omega}$, obtemos

$$
\left\{\begin{array}{l}
c \frac{\partial v}{\partial x}+c\left(1+d_{x} \frac{\imath}{\omega}\right) \frac{\partial w}{\partial y}=-\imath \omega u+d_{x} u \\
c \frac{\partial u}{\partial x}=-\imath \omega v+d_{x} v
\end{array} .\right.
$$

A segunda equação pode ser expressa no domínio do tempo diretamente. Na primeira, temos um termo dependente diretamente de $\frac{\imath}{\omega}$. Para transformá-lo para o domínio do tempo, observamos que, uma vez que a multiplicação por - $\imath \omega$ representa diferenciação no tempo, a divisão por esse valor pode ser compreendida como integração no tempo. Dessa forma, o termo $d_{x} \frac{\imath}{\omega} \frac{\partial w}{\partial y}$ é a integral no domínio do tempo de uma função auxiliar $\Psi$. Escrevemos então, adicionalmente ao sistema dado, a seguinte equação:

$$
-\imath \omega \Psi=c \cdot d_{x} \frac{\partial w}{\partial y}
$$

Substituindo na primeira equação, obtemos o sistema para a PML para a equação bidimensional:

$$
\left\{\begin{array}{l}
\frac{\partial u}{\partial t}=c \frac{\partial v}{\partial x}+c \frac{\partial w}{\partial y}+\Psi-d_{x} u \\
\frac{\partial v}{\partial t}=c \frac{\partial u}{\partial x}-d_{x} v \\
\frac{\partial w}{\partial t}=c \frac{\partial u}{\partial y} \\
\frac{\partial \Psi}{\partial t}=c \cdot d_{x} \frac{\partial w}{\partial y}
\end{array}\right.
$$

Para realizar o amortecimento simultaneamente nas direções $x$ e $y$, podemos aplicar as transformações para as duas direções de forma concomitante. Assim, o sistema (2.23) é escrito da seguinte 
forma:

$$
\left\{\begin{array}{l}
\frac{\partial u}{\partial t}=c \frac{1}{1+\imath \frac{d_{x}(x)}{\omega}} \frac{\partial v}{\partial x}+c \frac{1}{1+\imath \frac{d_{y}(y)}{\omega}} \frac{\partial w}{\partial y}=-i \omega u \\
\frac{\partial v}{\partial t}=c \frac{1}{1+\imath \frac{d_{x}(x)}{\omega}} \frac{\partial u}{\partial x} \\
\frac{\partial w}{\partial t}=c \frac{1}{1+\imath \frac{d_{y}(y)}{\omega}} \frac{\partial u}{\partial y}
\end{array} .\right.
$$

Multiplicando a primeira equação do sistema acima por $\left(1+d_{x} \frac{\imath}{\omega}\right)\left(1+d_{y} \frac{\imath}{\omega}\right)$, obtemos

$$
c\left(1+d_{y} \frac{\imath}{\omega}\right) \frac{\partial v}{\partial x}+c\left(1+d_{x} \frac{\imath}{\omega}\right) \frac{\partial w}{\partial y}=-\left(1+d_{x} \frac{\imath}{\omega}\right)\left(1+d_{y} \frac{\imath}{\omega}\right) \imath \omega u,
$$

ou ainda,

$$
b \frac{\partial v}{\partial x}+b \cdot d_{y} \frac{\imath}{\omega} \frac{\partial v}{\partial x}+b \frac{\partial w}{\partial y}+b \cdot d_{x} \frac{\imath}{\omega} \frac{\partial w}{\partial y}=-\imath \omega u+\left(d_{x}+d_{y}\right) u+\frac{\imath}{\omega} d_{x} d_{y} u
$$

Nesse caso, temos três termos explicitamente dependentes de $\frac{\imath}{\omega}$, de modo que, novamente, acrescentamos funções auxiliares $\Psi_{1}, \Psi_{2}, \Psi_{3}$ tais que

$$
\begin{aligned}
& -\imath \omega \Psi_{1}=d_{x} d_{y} u \\
& -\imath \omega \Psi_{2}=c \cdot d_{y} \frac{\partial v}{\partial x} \\
& -\imath \omega \Psi_{3}=c \cdot d_{x} \frac{\partial w}{\partial y} .
\end{aligned}
$$

As demais equações podem ser transformadas diretamente para o domínio do tempo sem a inserção de funções auxiliares, de modo que a PML para o sistema de primeira ordem em duas dimensões pode ser escrito como

$$
\left\{\begin{array}{l}
\frac{\partial u}{\partial t}=c \frac{\partial v}{\partial x}+c \frac{\partial w}{\partial y}+\Psi_{1}+\Psi_{2}+\Psi_{3}-\left(d_{x}+d_{y}\right) u \\
\frac{\partial v}{\partial t}=c \frac{\partial u}{\partial x}-d_{x} v \\
\frac{\partial w}{\partial t}=c \frac{\partial u}{\partial y}-d_{y} w \\
\frac{\partial \Psi_{1}}{\partial t}=u \\
\frac{\partial \Psi_{2}}{\partial t}=c \cdot d_{y} \frac{\partial v}{\partial x} \\
\frac{\partial \Psi_{3}}{\partial t}=c \cdot d_{x} \frac{\partial w}{\partial y}
\end{array}\right.
$$

Para a obtenção do sistema dado, assumimos $F \equiv 0$, uma vez que isso ocorrerá, em geral, na camada de amortecimento. Caso o termo de fonte $F$ não seja nulo para todos os pontos do domínio no qual se deseja calcular a solução, podemos acrescentar ao segundo membro da primeira equação do sistema (2.26) a parcela $G(x, y, t)=\int_{0}^{t} F(x, y, t)$.

Discretização: Para resolver numericamente tais equações em um domínio $\Omega_{2}$, definimos uma malha staggered, na qual as funções $u, \Psi_{1}, \Psi_{2}, \Psi_{3}$ são definidas em pontos $\left(x_{i}, y_{j}\right)$ tais que $x_{i}:=$ $x_{I}+i \Delta x$ e $y_{j}:=y_{I}+j \Delta y$ e as funções $v$ e $w$ são definidas em pontos $\left(x_{i+\frac{1}{2}}, y_{j}\right)$ e $\left(x_{i}, y_{j+\frac{1}{2}}\right)$ respectivamente, de modo que $x_{i+\frac{1}{2}}:=x_{I}+\left(i+\frac{1}{2}\right) \Delta x$ e $y_{j+\frac{1}{2}}:=y_{I}+\left(j+\frac{1}{2}\right) \Delta y$.

Denotando por $u^{n+\frac{1}{2}}:=u^{\left(n+\frac{1}{2}\right) \Delta t}$, a versão discreta adotada neste trabalho para a condição 
(2.26) é dada por

$$
\left\{\begin{array}{l}
\frac{\Psi_{1_{i, j}}^{n+\frac{1}{2}}-\Psi_{i_{i, j}}^{n-\frac{1}{2}}}{\Delta t}=u_{i, j}^{n} \\
\frac{\Psi_{2_{i, j}}^{n+1}-\Psi_{2_{i, j}}^{n}}{\Delta t}=c \cdot d_{y} \frac{v_{i+\frac{1}{2}, j}^{n+\frac{1}{2}}-v_{i-\frac{1}{2}, j}^{n+\frac{1}{2}}}{\Delta x} \\
\frac{\Psi_{3_{i, j}}^{n+1}-\Psi_{3_{i, j}}^{n}}{\Delta t}=c \cdot d_{x} \frac{w_{i, j+\frac{1}{2}}^{n+\frac{1}{2}}-w_{i, j-\frac{1}{2}}^{n+\frac{1}{2}}}{\Delta y} \\
\frac{v_{i+\frac{1}{2}, j}^{n+\frac{1}{2}}-v_{i+\frac{1}{2}, j}^{n-\frac{1}{2}}}{\Delta t}=c \frac{u_{i+1, j}^{n}-u_{i, j}^{n}}{\Delta x}-d_{x} v_{i+\frac{1}{2}, j}^{n} \\
\frac{w_{i, j+\frac{1}{2}}^{n+\frac{1}{2}}-w_{i, j+\frac{1}{2}}^{n-\frac{1}{2}}}{\Delta t}=c \frac{u_{i, j+1}^{n}-u_{i, j}^{n}}{\Delta y}-d_{y} w_{i, j+\frac{1}{2}}^{n+\frac{1}{2}} \\
\frac{u_{i, j}^{n+1}-u_{i, j}^{n}}{\Delta t}=c \frac{v_{i+\frac{1}{2}, j}^{n+\frac{1}{2}}-v_{i-\frac{1}{2}, j}^{n+\frac{1}{2}}}{\Delta x}+c \frac{w_{i, j+\frac{1}{2}}^{n+\frac{1}{2}}-w_{i, j-\frac{1}{2}}^{n+\frac{1}{2}}}{\Delta y}+\Psi_{1_{i, j}}^{n+\frac{1}{2}}+\Psi_{2_{i, j}}^{n+\frac{1}{2}}+\Psi_{i_{i, j}}^{n+\frac{1}{2}}-\left(d_{x}+d_{y}\right) u_{i, j}^{n+\frac{1}{2}}
\end{array}\right.
$$

onde $\Psi_{k}^{n+\frac{1}{2}}=\frac{\Psi_{k}^{n+1}+\Psi_{k}^{n}}{2}, k=2,3, u_{i, j}^{n+\frac{1}{2}}=\frac{u_{i, j}^{n+1}+u_{i, j}^{n}}{2}, v_{i, j}^{n}=\frac{v_{i, j}^{n+\frac{1}{2}}+v_{i, j}^{n-\frac{1}{2}}}{2}, w_{i, j}^{n}=\frac{w_{i, j}^{n+\frac{1}{2}}+w_{i, j}^{n-\frac{1}{2}}}{2}$.

Para o caso unidimensional, podemos fazer

$$
\begin{aligned}
\frac{u_{i}^{n+1}-u_{i}^{n}}{\Delta t} & =c \frac{v_{i+\frac{1}{2}}^{n+\frac{1}{2}}-v_{i-\frac{1}{2}}^{n+\frac{1}{2}}}{\Delta x}-d_{x} u_{i}^{n+\frac{1}{2}} \\
\frac{v_{i+\frac{1}{2}}^{n+\frac{1}{2}}-v_{i+\frac{1}{2}}^{n-\frac{1}{2}}}{\Delta t} & =c \frac{u_{i+1}^{n}-u_{i}^{n}}{\Delta x}-d_{x} v_{i+\frac{1}{2}}^{n}
\end{aligned} .
$$

Até aqui, foram consideradas somente as ondas com $k_{x} \in \mathbb{R}$. Podemos considerar ainda o caso em que $k_{x} \in \mathbb{C}$. Como vimos, dada uma solução da forma $e^{\imath(\mathbf{k} \cdot \mathbf{x}-\omega t)}, k_{x}, k_{y}$ e $\omega$ devem satisfazer $k_{x}=\sqrt{\frac{\omega^{2}}{c^{2}}-k_{y}^{2}}$. Para $k_{y}$ suficientemente grande, $k_{x}$ é puramente imaginário e, para $\operatorname{Im} k_{x}>0$, o termo $e^{\imath k_{x} x}$ decai exponencialmente. Para acelerar esse decaimento, podemos tomar $d_{x} \in \mathbb{C}$, realizando o alongamento da coordenada real e aumentando, dessa forma, a taxa de decaimento da solução.

A função $d_{x}(x)$ é frequentemente adotada da seguinte forma:

$$
d_{x}(x)=\frac{3 c}{2 \delta} \log \left(\frac{1}{R}\right)\left(\frac{x}{\delta}\right)^{2},
$$

onde $\delta$ é a espessura da PML e R é o coeficiente de reflexão.

\section{PML para a equação de segunda ordem na variável temporal}

Outra formulação possível para a PML envolve a aplicação de camadas de amortecimento diretamente à equação de segunda ordem. Isso pode ser feito conforme o desenvolvimento realizado por [GS10], que apresentamos a seguir.

Consideremos novamente a equação

$$
\begin{array}{r}
\frac{\partial^{2} u}{\partial t^{2}}=c^{2}\left(\frac{\partial^{2} u}{\partial x^{2}}+\frac{\partial^{2} u}{\partial y^{2}}\right)+F(x, y, t) \\
u(x, y, 0)=u_{0} \\
\frac{\partial u}{\partial t}(x, y, 0)=v_{0}
\end{array}
$$


definida em um domínio $\Omega_{2}=\left[x_{I}, x_{F}\right] \times\left[y_{I}, y_{F}\right]$. Seja $\hat{u}$ a Transformada de Laplace de $u$, dada por $\hat{u}=\int_{0}^{\infty} e^{s t} u(x, y, t) d t, s \in \mathbb{C}$. Da equação $(2.28)$, temos que, fora de $\Omega_{2}$, $\hat{u}$ satisfaz a equação de Helmholtz

$$
s^{2} \hat{u}=\frac{\partial}{\partial x}\left(c^{2} \frac{\partial \hat{u}}{\partial x}\right)+\frac{\partial}{\partial y}\left(c^{2} \frac{\partial \hat{u}}{\partial y}\right) .
$$

Definimos a mudança de variáveis

$$
\begin{aligned}
& x \rightarrow \tilde{x}:=x+\frac{1}{s} \int_{0}^{x} d_{x}(s) d s \\
& y \rightarrow \tilde{y}:=y+\frac{1}{s} \int_{0}^{y} d_{y}(s) d s
\end{aligned}
$$

de modo que $d_{x}$ e $d_{y}$ sejam, assim como antes, perfis de amortecimento tais que, para $(x, y) \in \Omega_{2}$, $d_{x}(x)=d_{y}(y)=0$. Fazendo com que $\hat{u}$ satisfaça a equação de Helmholtz também nas variáveis $\tilde{x}, \tilde{y}$, temos que a solução permanece inalterada em $\Omega_{2}$, mas decai exponencialmente na camada de amortecimento. Da mudança de variáveis apresentada em (2.30), temos

$$
\begin{aligned}
\frac{\partial}{\partial \tilde{x}} & =\frac{s}{s+d_{x}} \frac{\partial}{\partial x} \\
\frac{\partial}{\partial \tilde{y}} & =\frac{s}{s+d_{y}} \frac{\partial}{\partial y} .
\end{aligned}
$$

Definimos as variáveis $\gamma_{k}, k=x, y$, como $\gamma_{k}:=1+\frac{d_{k}}{s}$ e, assim, podemos reescrever a equação (2.28) nas variáveis $\tilde{x}, \tilde{y}$ como segue:

$$
s^{2} \hat{u}=\frac{\partial}{\partial \tilde{x}}\left(c^{2} \frac{\partial \hat{u}}{\partial \tilde{x}}\right)+\frac{\partial}{\partial \tilde{y}}\left(c^{2} \frac{\partial \hat{u}}{\partial \tilde{y}}\right),
$$

e então,

$$
s^{2} \hat{u}=\frac{1}{\gamma_{x}} \frac{\partial}{\partial x}\left(c^{2} \frac{1}{\gamma_{x}} \frac{\partial \hat{u}}{\partial x}\right)+\frac{1}{\gamma_{y}} \frac{\partial}{\partial y}\left(c^{2} \frac{1}{\gamma_{y}} \frac{\partial \hat{u}}{\partial y}\right) .
$$

Multiplicando ambos os membros de (2.31) por $\gamma_{x} \gamma_{y}$, temos

$$
s^{2} \gamma_{x} \gamma_{y} \hat{u}=\frac{\partial}{\partial x}\left(c^{2} \frac{\gamma_{y}}{\gamma_{x}} \frac{\partial \hat{u}}{\partial x}\right)+\frac{\partial}{\partial y}\left(c^{2} \frac{\gamma_{x}}{\gamma_{y}} \frac{\partial \hat{u}}{\partial y}\right) .
$$

Pelas definições de $\gamma_{x}$ e $\gamma_{y}$, podemos obter

$$
\begin{gathered}
\frac{\gamma_{x}}{\gamma_{y}}=\frac{s+d_{x}}{s+d_{y}}=1+\frac{d_{x}-d_{y}}{s+d_{y}} \\
\frac{\gamma_{y}}{\gamma_{x}}=\frac{s+d_{y}}{s+d_{x}}=1+\frac{d_{y}-d_{x}}{s+d_{x}} \\
\gamma_{x} \gamma_{y}=\frac{\left(s+d_{x}\right)\left(s+d_{y}\right)}{s^{2}}=\frac{s^{2}+s\left(d_{x}+d_{y}\right)+d_{x} d_{y}}{s^{2}} .
\end{gathered}
$$

Introduzindo essas relações na equação (2.32), obtemos 


$$
\begin{gathered}
{\left[s^{2}+s\left(d_{x}+d_{y}\right)+d_{x} d_{y}\right] \hat{u}=\frac{\partial}{\partial x}\left[c^{2}\left(1+\frac{d_{y}-d_{x}}{s+d_{x}}\right) \frac{\partial \hat{u}}{\partial x}\right]+\frac{\partial}{\partial y}\left[c^{2}\left(1+\frac{d_{x}-d_{y}}{s+d_{y}}\right) \frac{\partial \hat{u}}{\partial y}\right] .} \\
{\left[s^{2}+s\left(d_{x}+d_{y}\right)+d_{x} d_{y}\right] \hat{u}=\frac{\partial}{\partial x}\left(c^{2} \frac{\partial \hat{u}}{\partial x}\right)+\frac{\partial}{\partial y}\left(c^{2} \frac{\partial \hat{u}}{\partial y}\right)+\frac{\partial}{\partial x}\left(c^{2} \frac{d_{y}-d_{x}}{s+d_{x}} \frac{\partial \hat{u}}{\partial x}\right)+\frac{\partial}{\partial y}\left(c^{2} \frac{d_{x}-d_{y}}{s+d_{y}} \frac{\partial \hat{u}}{\partial x}\right) .}
\end{gathered}
$$

Definimos, ainda, duas funções auxiliares $\phi_{1}$ e $\phi_{2}$, dadas pelas seguintes relações:

$$
\begin{aligned}
& \hat{\phi}_{1}=c^{2} \frac{d_{y}-d_{x}}{s+d_{x}} \frac{\partial \hat{u}}{\partial x} \Rightarrow\left(s+d_{x}\right) \hat{\phi}_{1}=c^{2}\left(d_{y}-d_{x}\right) \frac{\partial \hat{u}}{\partial x} \\
& \hat{\phi}_{2}=c^{2} \frac{d_{x}-d_{y}}{s+d_{y}} \frac{\partial \hat{u}}{\partial y} \Rightarrow\left(s+d_{y}\right) \hat{\phi}_{2}=c^{2}\left(d_{x}-d_{y}\right) \frac{\partial \hat{u}}{\partial y} .
\end{aligned}
$$

Por fim, utilizando essas funções no sistema obtido, temos

$$
\begin{aligned}
& \frac{\partial^{2} u}{\partial t^{2}}+\left(d_{x}+d_{y}\right) \frac{\partial u}{\partial t}+d_{x} d_{y} u=c^{2}\left(\frac{\partial^{2} u}{\partial x^{2}}+\frac{\partial^{2} u}{\partial y^{2}}\right)+\frac{\partial \phi_{1}}{\partial x}+\frac{\partial \phi_{2}}{\partial y}+F(x, y, t) \\
& \frac{\partial \phi_{1}}{\partial t}=-d_{x} \phi_{1}+c^{2}\left(d_{y}-d_{x}\right) \frac{\partial u}{\partial x} \\
& \frac{\partial \phi_{2}}{\partial t}=-d_{y} \phi_{2}+c^{2}\left(d_{x}-d_{y}\right) \frac{\partial u}{\partial y} .
\end{aligned}
$$

Em uma dimensão, a PML sugerida assume a seguinte forma (obtida desconsiderando a segunda dimensão espacial na condição acima):

$$
\begin{array}{r}
\frac{\partial^{2} u}{\partial t^{2}}+d_{x} \frac{\partial u}{\partial t}=c^{2}\left(\frac{\partial^{2} u}{\partial x^{2}}\right)+\frac{\partial \phi_{1}}{\partial x}+F(x, t) \\
\frac{\partial \phi_{1}}{\partial t}=-d_{x} \phi_{1}+c^{2}\left(-d_{x}\right) \frac{\partial u}{\partial x} .
\end{array}
$$

Discretização: A discretização proposta por Grote em [GS10] para a PML desenvolvida anteriormente é a seguinte: 


$$
\begin{aligned}
& \frac{u_{i, j}^{n+1}-2 u_{i, j}^{n}+u_{i, j}^{n-1}}{\Delta t^{2}}+\left[d_{x}\left(x_{i}\right)+d_{y}\left(y_{j}\right)\right] \frac{u_{i, j}^{n+1}-u_{i, j}^{n-1}}{2 \Delta t}+d_{x}\left(x_{i}\right) d_{y}\left(y_{j}\right) u_{i, j}^{n}= \\
& c^{2}\left(\frac{u_{i+1, j}^{n}-2 u_{i, j}^{n}+u_{i-1, j}^{n}}{\Delta x^{2}}+\frac{u_{i, j+1}^{n}-2 u_{i, j}^{n}+u_{i, j-1}^{n}}{\Delta y^{2}}\right)+\frac{\widetilde{\Phi}_{1_{i+\frac{1}{2}, j}}^{n}-\widetilde{\Phi}_{1_{i-\frac{1}{2}, j}}^{n}}{\Delta x}+\frac{\widetilde{\Phi}_{2_{i, j+\frac{1}{2}}^{n}}-\widetilde{\Phi}_{2_{i, j-\frac{1}{2}}^{n}}}{\Delta y}+F_{i, j}^{n} \\
& \widetilde{\Phi}_{1_{i+\frac{1}{2}, j}^{n}}^{n}=\frac{1}{2}\left(\Phi_{1_{i+\frac{1}{2}, j-\frac{1}{2}}^{n}}^{n}+\Phi_{1_{i+\frac{1}{2}, j+\frac{1}{2}}^{n}}^{n}\right) \\
& \widetilde{\Phi}_{2_{i, j+\frac{1}{2}}^{n}}^{n}=\frac{1}{2}\left(\Phi_{2_{i+\frac{1}{2}, j+\frac{1}{2}}^{n}}+\Phi_{2_{i-\frac{1}{2}, j+\frac{1}{2}}^{n}}^{n}\right) \\
& \frac{\Phi_{1_{i+\frac{1}{2}, j+\frac{1}{2}}^{n+1}}^{n+1}-\Phi_{1_{i+\frac{1}{2}, j+\frac{1}{2}}^{n}}^{n}}{\Delta t}=-d_{x}\left(x_{i+\frac{1}{2}}\right) \frac{\Phi_{1_{i+\frac{1}{2}, j+\frac{1}{2}}^{n+1}}^{n+1}+\Phi_{1_{i+\frac{1}{2}, j+\frac{1}{2}}^{n}}^{2}}{2}+c^{2}\left[d_{y}\left(y_{j+\frac{1}{2}}\right)-d_{x}\left(x_{i+\frac{1}{2}}\right)\right] D_{x}^{h} u_{i+\frac{1}{2}, j+\frac{1}{2}}^{n+\frac{1}{2}} \\
& \frac{\Phi_{2_{i+\frac{1}{2}, j+\frac{1}{2}}^{n+1}}^{n+1} \Phi_{2_{i+\frac{1}{2}, j+\frac{1}{2}}^{n}}^{n}}{\Delta t}=-d_{y}\left(y_{j+\frac{1}{2}}\right) \frac{\Phi_{2_{i+\frac{1}{2}, j+\frac{1}{2}}^{n+1}}^{n+1}+\Phi_{2_{i+\frac{1}{2}, j+\frac{1}{2}}^{n}}^{2}}{2}+c^{2}\left[d_{x}\left(x_{i+\frac{1}{2}}\right)-d_{y}\left(y_{j+\frac{1}{2}}\right)\right] D_{y}^{h} u_{i+\frac{1}{2}, j+\frac{1}{2}}^{n+\frac{1}{2}} \\
& D_{x}^{h} u_{i+\frac{1}{2}, j+\frac{1}{2}}^{n+\frac{1}{2}}=\frac{1}{4}\left(\frac{u_{i+1, j}^{n+1}+u_{i+1, j+1}^{n+1}-u_{i, j}^{n+1}-u_{i, j+1}^{n+1}}{\Delta x}+\frac{u_{i+1, j}^{n}+u_{i+1, j+1}^{n}-u_{i, j}^{n}-u_{i, j+1}^{n}}{\Delta x}\right) \\
& D_{y}^{h} u_{i+\frac{1}{2}, j+\frac{1}{2}}^{n+\frac{1}{2}}=\frac{1}{4}\left(\frac{u_{i, j+1}^{n+1}+u_{i+1, j+1}^{n+1}-u_{i, j}^{n+1}-u_{i+1, j}^{n+1}}{\Delta y}+\frac{u_{i, j+1}^{n}+u_{i+1, j+1}^{n}-u_{i, j}^{n}-u_{i+1, j}^{n}}{\Delta y}\right) .
\end{aligned}
$$

Para a equação unidimensional, podemos obter uma versão análoga como segue:

$$
\begin{aligned}
& \frac{u_{i}^{n+1}-2 u_{i}^{n}+u_{i}^{n-1}}{\Delta t^{2}}+d_{x}\left(x_{i}\right) \frac{u_{i}^{n+1}-u_{i}^{n-1}}{2 \Delta t}=c^{2}\left(\frac{u_{i+1}^{n}-2 u_{i}^{n}+u_{i-1}^{n}}{\Delta x^{2}}\right)+\frac{\Phi_{1_{i+\frac{1}{2}}}^{n}-\Phi_{1_{i-\frac{1}{2}}}^{n}}{\Delta x}+F_{i}^{n} \\
& \frac{\Phi_{1_{i+\frac{1}{2}}^{n+1}}^{n}-\Phi_{1_{i+\frac{1}{2}}}^{n}}{\Delta t}=-d_{x}\left(x_{i+\frac{1}{2}}\right) \frac{\Phi_{1_{i+\frac{1}{2}}^{n+1}}^{n}+\Phi_{1_{i+\frac{1}{2}}}^{n}}{2}+c^{2}\left[-d_{x}\left(x_{i+\frac{1}{2}}\right)\right] D_{x}^{h} u_{i+\frac{1}{2}}^{n+\frac{1}{2}} \\
& D_{x}^{h} u_{i+\frac{1}{2}}^{n+\frac{1}{2}}=\frac{1}{2}\left(\frac{u_{i+1}^{n+1}-u_{i}^{n+1}}{\Delta x}+\frac{u_{i+1}^{n}-u_{i}^{n}}{\Delta x}\right) .
\end{aligned}
$$

\subsubsection{Condição de absorção híbrida}

Como vimos, as condições de contorno de absorção consistem em tratar os pontos no interior do domínio com a TWWE e, aos pontos do contorno, aplicar uma aproximação da OWWE. No entanto, a aplicação desse procedimento pode causar reflexões geradas pela mudança abrupta de tratamento entre o domínio e o contorno. Para solucionar esse problema, Liu e Sen [LS10] sugeriram a condição de contorno de absorção híbrida (HABC), que consiste em acrescentar em torno do domínio uma área de transição, na qual o campo é tratado como uma média ponderada da OWWE e da TWWE. O desenvolvimento apresentado a seguir baseia-se no trabalho de Ren e Liu [RL13], cuja abordagem considera a equação da onda elástica no domínio da frequência, trazendo uma discussão mais abrangente e incluindo apontamentos referentes às malhas escolhidas.

Consideremos a equação da onda bidimensional (2.2) definida em um domínio de interesse, que será denominado $A_{N+1}$. Em torno dessa região, acrescentamos uma faixa de espessura de $N$ pontos da grade e o conjunto de pontos acrescentados é composto por $N-1$ partes, cada qual denominada $A_{k}, k=N, \ldots, 2$, formando uma área de transição. Cada $A_{k}$ é composta pelos pontos $\left(x_{i}, y_{j}\right)$ tais que $y_{j} \in\left[y_{I}-k \Delta y, y_{F}+k \Delta y\right]$ e $x_{i}=x_{I}-k \Delta x$ ou $x_{i}=x_{F}+k \Delta x$, e também pelos pontos tais que $x_{i} \in\left[x_{I}-k \Delta x, x_{F}+k \Delta x\right]$ e $y_{j}=y_{I}-k \Delta y$ ou $y_{j}=y_{F}+k \Delta y$. Por fim, o contorno será denominado $A_{1}$. O domínio no qual realizaremos o tratamento pode ser ilustrado como segue: 


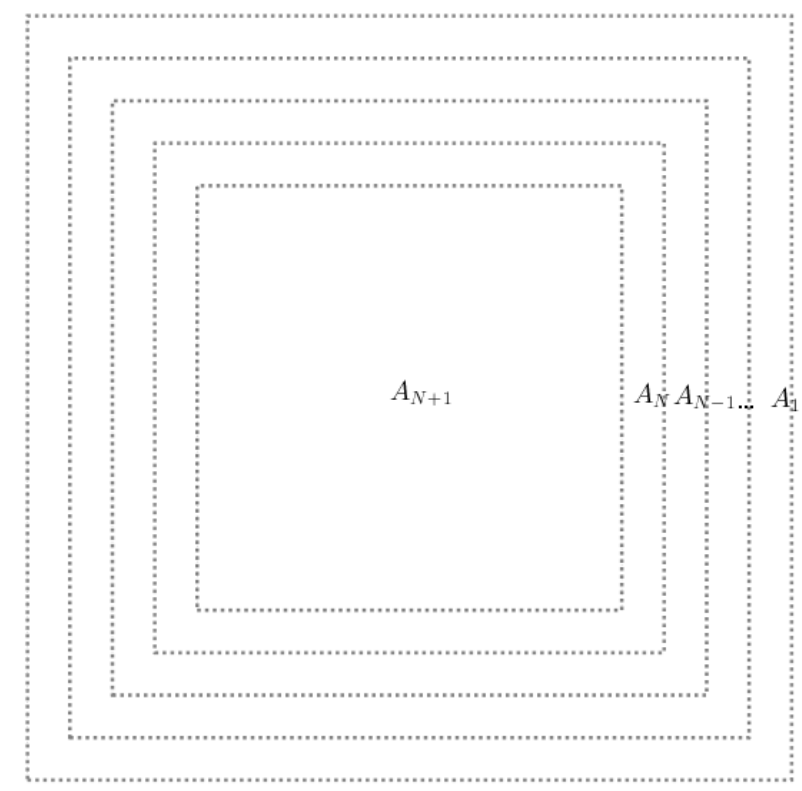

Figura 2.2: Domínio para aplicação da $H A B C$.

A aplicação da HABC é feita calculando a solução, em cada $A_{k}$, de acordo com a seguinte especificação:

$$
u_{i, j}^{n+1}=\left(1-\omega_{k}\right) u_{2_{i, j}}+\omega_{k} u_{1_{i, j}},
$$

onde $u_{2_{i, j}}$ e $u_{1_{i, j}}$ são as soluções calculadas em $\left(x_{i}, y_{j}\right)$ em $t_{n+1}$ aplicando a TWWE e a OWWE respectivamente, e os coeficientes $\omega_{k}$ são, na versão original da HABC, dados por $\omega_{k}=\frac{N+1-k}{N}$ para cada $A_{k}$. A partir dessa construção, podemos observar que para a região $N+1$, que é o domínio original, $\omega_{N+1}=0$, o que indica que nessa área a solução é calculada apenas com a TWWE. Já para $A_{1}, \omega_{1}=1$ e a solução é definida somente pela OWWE, que é aplicada de acordo com as formulações propostas por Engquist, Majda e Higdon.

A aplicação à equação da onda elástica pode ser realizada de forma análoga, qualquer que seja a formulação adotada. Porém, é importante observar a adequação das malhas à formulação escolhida. Mais detalhes acerca da escolha entre malhas regulares e staggered grids e sobre a aplicação da HABC à equação da onda elástica podem ser encontrados em [RL13].

Uma versão modificada para a HABC envolve a alteração dos coeficientes $\omega_{k}$. Em [LS18], é sugerida a seguinte forma para os coeficientes:

$$
\omega_{k}=\left\{\begin{array}{l}
1, \text { se } 1<k \leq M+1 \\
\left(\frac{N+1-k}{N-M}\right)^{\alpha}, \text { se } M+2 \leq k \leq N \\
0, \text { se } k=N+1
\end{array} .\right.
$$

Discretização: A discretização das condições depende da condição de absorção utilizada. A obtenção de $u_{2_{i, j}}$ pode ser feita com a discretização padrão para a equação da onda de segunda ordem. Já $u_{1_{i, j}}$ pode ser obtida utilizando, por exemplo, as discretizações (2.12), (2.13) e (2.17), sugeridas para as condições A1, A2 e para os operadores de Higdon. A aplicação da HABC para o caso bidimensional com a condição A1 para o contorno direito em cada região $A_{k}$, por exemplo, é feita da seguinte forma:

1. Para o domínio original $A_{N+1}$, calcula-se

$$
\frac{u_{i, j}^{n+1}}{\Delta t^{2}}=\frac{2 u_{i, j}^{n}-u_{i, j}^{n-1}}{\Delta t^{2}}+c^{2} \frac{u_{i+1, j}^{n}-2 u_{i, j}^{n}+u_{i-1, j}^{n}}{\Delta x^{2}}+c^{2} \frac{u_{i, j+1}^{n}-2 u_{i, j}^{n}+u_{i, j-1}^{n}}{\Delta y^{2}},
$$


2. Para as regiões $A_{k}, \operatorname{com} k=2, \ldots, N$,

- $\frac{u_{2_{i, j}+1}^{n}}{\Delta t^{2}}=\frac{2 u_{i, j}^{n}-u_{i, j}^{n-1}}{\Delta t^{2}}+c^{2} \frac{u_{i+1, j}^{n}-2 u_{i, j}^{n}+u_{i-1, j}^{n}}{\Delta x^{2}}+c^{2} \frac{u_{i, j+1}^{n}-2 u_{i, j}^{n}+u_{i, j-1}^{n}}{\Delta y^{2}}$,

- $c \frac{u_{1_{i, j}}^{n+1}}{\Delta x}+\frac{u_{1_{i, j}}^{n+1}}{\Delta t}=-c\left(\frac{u_{i, j}^{n}-u_{i-1, j}^{n}}{\Delta x}-\frac{u_{i-1, j}^{n+1}}{\Delta x}\right)-\left(-\frac{u_{i, j}^{n}}{\Delta t}+\frac{u_{i-1, j}^{n+1}-u_{i-1, j}^{n}}{\Delta t}\right)$,

- $u_{i, j}^{n+1}=\left(1-\omega_{k}\right) u_{2_{i, j}}^{n+1}+\omega_{k} u_{1_{i, j}}^{n+1}$,

3. Para a região $A_{1}$,

$$
c \frac{u_{i, j}^{n+1}}{\Delta x}+\frac{u_{i, j}^{n+1}}{\Delta t}=-c\left(\frac{u_{i, j}^{n}-u_{i-1, j}^{n}}{\Delta x}-\frac{u_{i-1, j}^{n+1}}{\Delta x}\right)-\left(-\frac{u_{i, j}^{n}}{\Delta t}+\frac{u_{i-1, j}^{n+1}-u_{i-1, j}^{n}}{\Delta t}\right) .
$$

Para o caso unidimensional, o procedimento é análogo e, para os demais contornos, são aplicados os operadores correspondentes para a condição de absorção adotada. 


\section{Capítulo 3}

\section{Resultados numéricos}

Neste capítulo, apresentamos resultados da aplicação das condições de contorno abordadas no capítulo anterior ao tratamento numérico das equações unidimensional e bidimensional da onda acústica. Realizamos também considerações em relação ao número de CFL, o refinamento da malha e outros aspectos relacionados à aplicação dos métodos, e verificamos sua influência na eficácia dos mesmos. Para cada equação, apresentamos dois testes distintos, sendo um com termo de fonte não nulo e um com condição inicial não nula. O objetivo destes testes é apresentar a aplicação dos métodos a situações distintas e permitir a verificação de aspectos diversos em relação aos mesmos. Apresentamos, ainda, um exemplo da equação da onda bidimensional em um meio com duas velocidades de propagação distintas.

Os resultados a seguir são referentes à aplicação do método de Cerjan, do método de Sochacki com os amortecedores linear, cúbico e expoente, dos métodos A1 e A2 de Majda e Engquist e da condição de Higdon de primeira e segunda ordem (a condição A2 é apresentada somente para o caso bidimensional), das PMLs formuladas para a equação escrita como sistema de primeira ordem e como equação de segunda ordem (denominadas, em alguns casos, PML 1 e PML 2, respectivamente) e da HABC em suas formas original e modificada (às quais nos referimos como HABC 1 e HABC 2, quando necessário) associadas às condições A1, A2 e à condição de Higdon de segunda ordem. Incluímos também a condição de Dirichlet que, embora não possua propriedades de absorção ou amortecimento, pode ser utilizada como parâmetro para avaliação das demais condições propostas.

Os resultados foram obtidos por meio de implementação em Python; detalhes de como essa linguagem pode ser utilizada de modo geral (e especificamente para os propósitos deste trabalho) podem ser encontradas em [LL11] e [LL17].

\subsection{Equação da onda unidimensional - Exemplo 1}

Neste exemplo, resolvemos a equação

$$
\begin{aligned}
u_{t t}-u_{x x} & =f(t) \delta(x-0.5), x \in[0,1] \\
u(x, 0) & =0 \\
u_{t}(x, 0) & =0
\end{aligned}
$$

onde

$$
f(t)=1-1800 \pi^{2} t^{2} e^{-900 \pi^{2} t^{2}}
$$

$\mathrm{e}$

$$
\delta(x)=\left\{\begin{array}{l}
1, \text { se } x=0, \\
0, \text { caso contrário }
\end{array}\right.
$$

Neste teste, as condições iniciais são nulas e o termo de fonte atua pontualmente no meio do domínio. O tempo de propagação da onda até a borda é igual a 0.5 , de modo que, para $t_{n}<0.5$, 
não são verificados efeitos do truncamento do domínio. Os valores adotados para esse teste são:

- Tempo total de simulação: $T=2$;

- Malha de 100 pontos no espaço;

- $\Delta x=0.01, \Delta t=0.005, \lambda=c \frac{\Delta t}{\Delta x}=0.5$.

Nas figuras abaixo, temos as representações gráficas de $f(t)$ e da solução tomada como referência no decorrer do tempo, simulada numericamente com os valores mencionados:

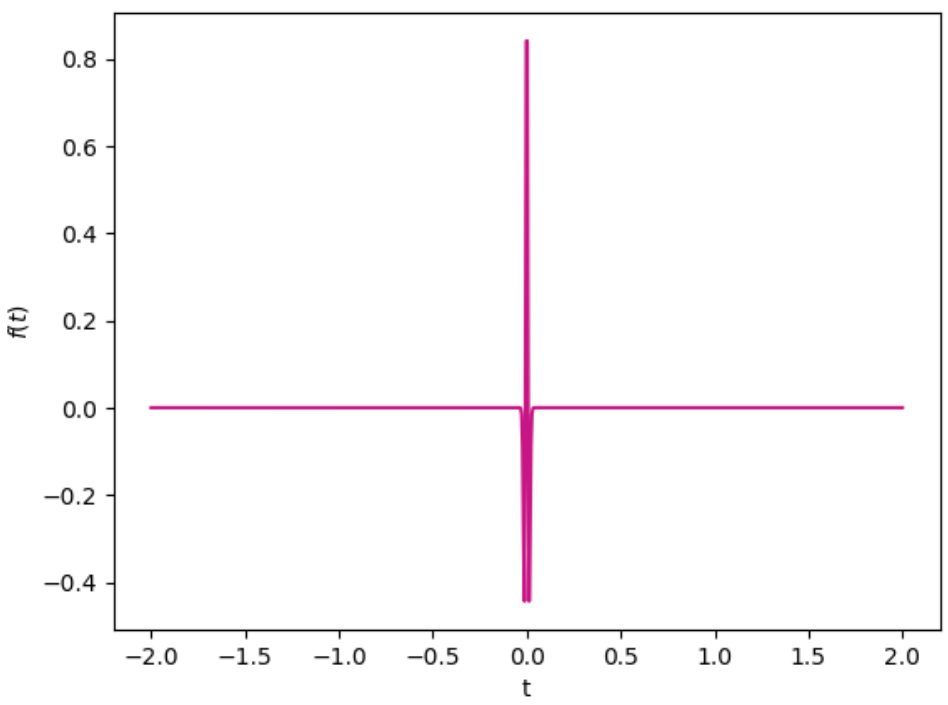

Figura 3.1: Gráfico de $f(t)$

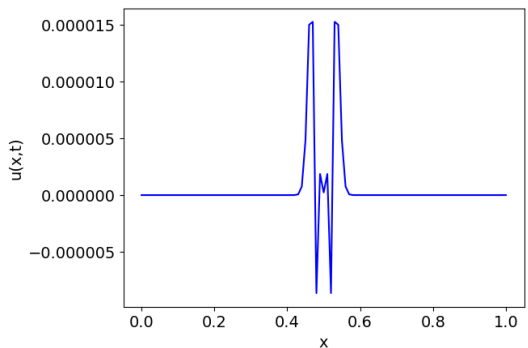

(a) $t=0.05$

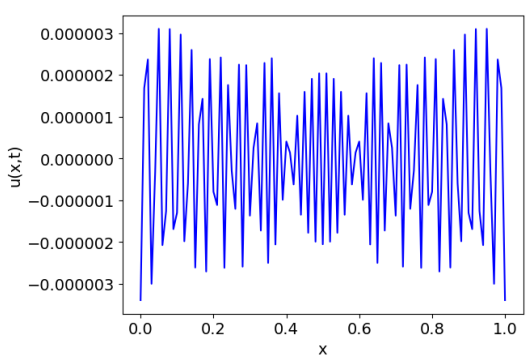

(d) $t=0.75$

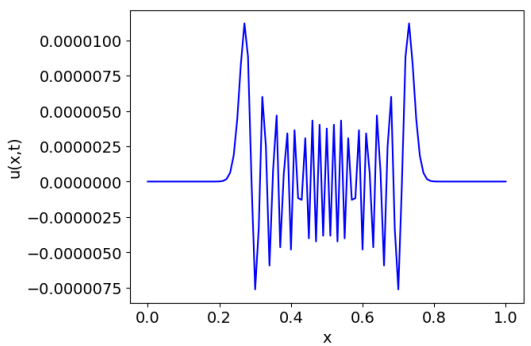

(b) $t=0.25$

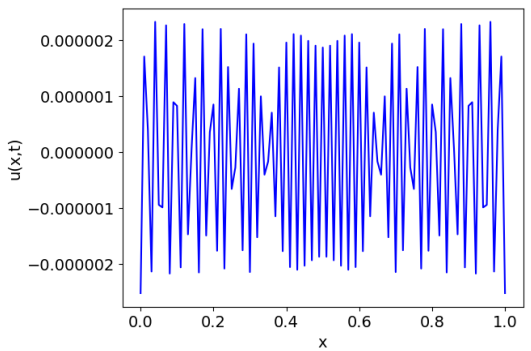

(e) $t=1$

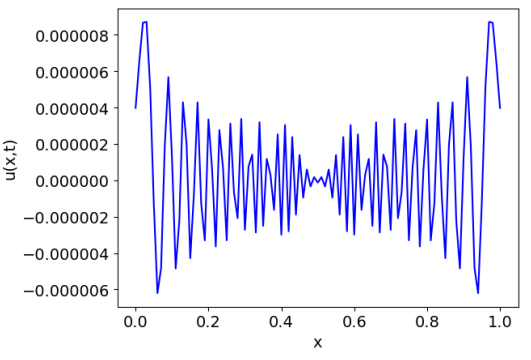

(c) $t=0.5$

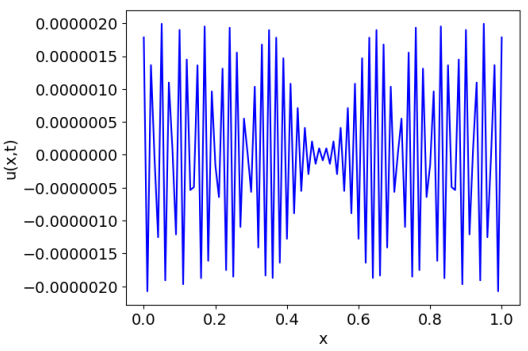

(f) $t=1.25$

Figura 3.2: Solução de referência no decorrer do tempo para o primeiro exemplo com a equação da onda unidimensional.

Os resultados são apresentados por meio de gráficos unidimensionais da solução obtida para cada condição de contorno, acompanhado pela solução de referência, conforme a figura 3.2, simulada a 
partir da resolução da equação em um domínio estendido de modo que as reflexões causadas pelo truncamento do domínio ainda não sejam visíveis no tempo de observação. Utilizamos ainda duas medidas de erro no instante $t_{n}$ dadas por

$$
\frac{\left\|e^{n}\right\|_{\infty}}{\left\|u^{n}\right\|_{\infty}}=\frac{\sup _{i}\left\{\left|u_{i}^{*^{n}}-u_{i}^{n}\right|\right\}}{\sup _{i}\left\{\left|u_{i}^{*^{n}}\right|\right\}},\left\|e^{n}\right\|_{2}=\left(\frac{\sum_{i=0}^{N}\left|u_{i}^{*^{n}}-u_{i}^{n}\right|^{2}}{N}\right)^{\frac{1}{2}},
$$

onde $u_{i}^{*^{n}}$ é o valor da solução tomada como referência e $N$ é o número de pontos do domínio computacional.

Na próxima seção são apresentados os resultados obtidos com $\lambda=0.5 \mathrm{e}$, posteriormente, incluímos os erros obtidos para outros valores de $\lambda$. Por simplicidade, denotaremos por $L$ o número de pontos da malha acrescentados a cada extremidade do domínio considerado nas camadas de amortecimento, e o mesmo valor será usado nas duas extremidades.

Os parâmetros utilizados para a aplicação das condições ao teste foram os seguintes:

- Para todos os casos, $L=20$, e a espessura da camada de amortecimento é $\delta=20 \cdot \Delta x=0.2$;

- Condição de Cerjan: $\tilde{\lambda}=0.0125$;

- HABC 2: $M=2, \alpha=1.5+0.07(L-M)$;

- Condição de Higdon de primeira ordem: $\alpha=\frac{\pi}{8}, a=b=0.5$;

- Condição de Higdon de segunda ordem: $\alpha_{1}=\frac{\pi}{4}, \alpha_{2}=\frac{\pi}{3}, a_{1}=a_{2}=b_{1}=b_{2}=0.5$;

- PML para o sistema de primeira ordem: $R=10^{-3}$,

$$
d(x)=\left\{\begin{array}{l}
\frac{3}{40 \Delta x} \ln \left(\frac{1}{0.001}\right)\left(\frac{x}{20 \Delta x}\right)^{2}, \text { se } x<0 \\
\frac{3}{40 \Delta x} \ln \left(\frac{1}{0.001}\right)\left(\frac{x-1}{20 \Delta x}\right)^{2}, \text { se } x>1
\end{array}\right.
$$

- PML para a equação de segunda ordem: $R=10^{-7}$,

$$
d(x)=\left\{\begin{array}{l}
\frac{3}{40 \Delta x} \ln \left(\frac{1}{10^{-7}}\right)\left(\frac{x}{20 \Delta x}\right)^{2}, \text { se } x<0 \\
\frac{3}{40 \Delta x} \ln \left(\frac{1}{10^{-7}}\right)\left(\frac{x-1}{20 \Delta x}\right)^{2}, \text { se } x>1
\end{array}\right.
$$

- Condição de Sochacki com amortecedor cúbico: $S=10^{7.5}$;

- Condição de Sochacki com amortecedor expoente: $S=10$;

- Condição de Sochacki com amortecedor linear: $S=10^{3.25}$. 


\subsubsection{Resultados para $\lambda=0.5$}

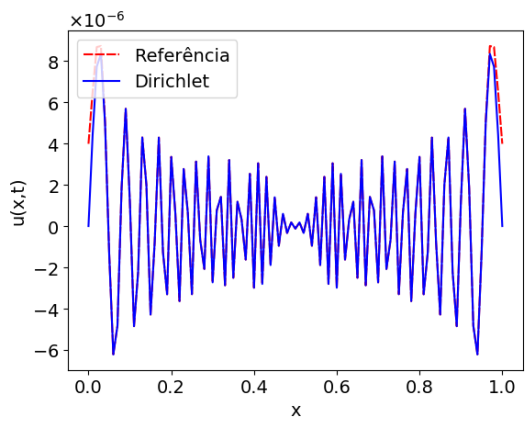

(a) $t=0.5$

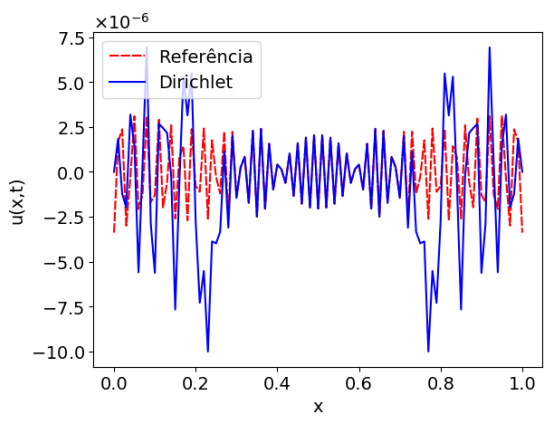

(b) $t=0.75$

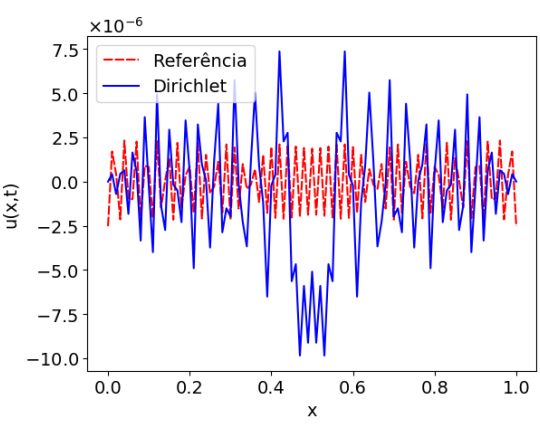

(c) $t=1$

Figura 3.3: Condição de Dirichlet $u=0$ aplicada ao primeiro exemplo unidimensional.

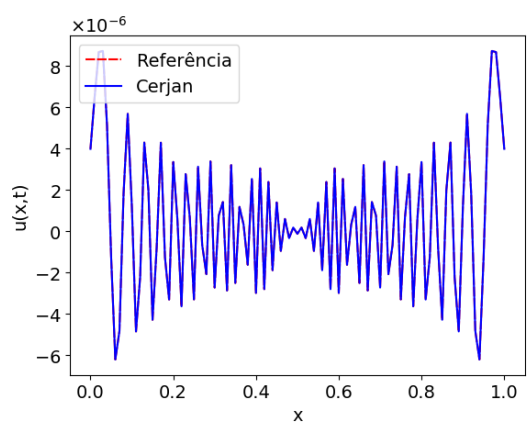

(a) $t=0.5$

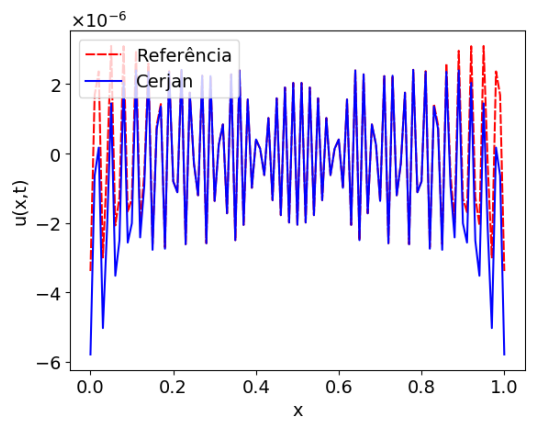

(b) $t=0.75$

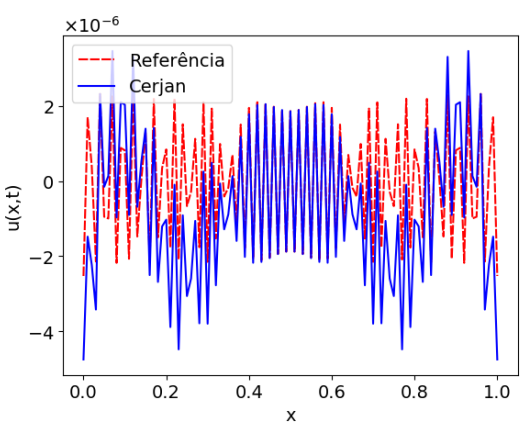

(c) $t=1$

Figura 3.4: Condição de Cerjan, com $\lambda=0.0125$, aplicada ao primeiro exemplo unidimensional.

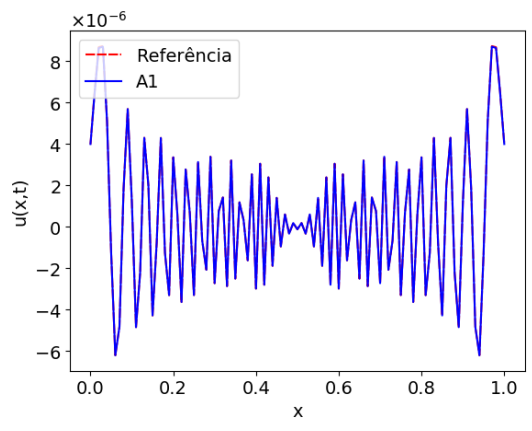

(a) $t=0.5$

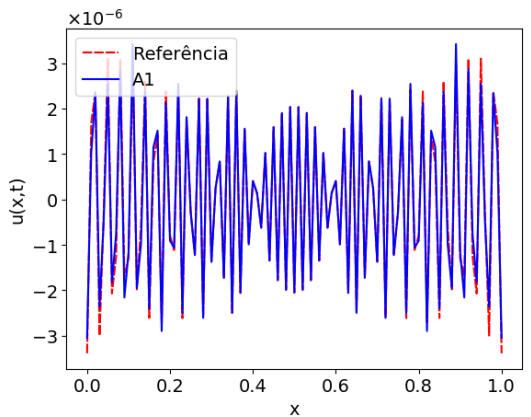

(b) $t=0.75$

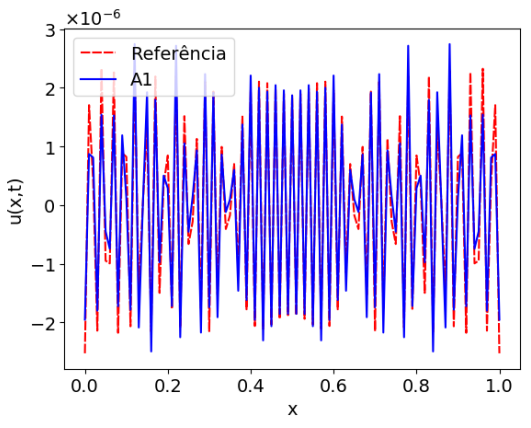

(c) $t=1$

Figura 3.5: Condição A1 aplicada ao primeiro exemplo unidimensional. 


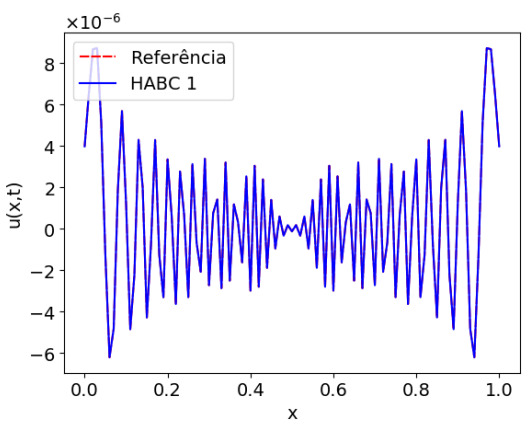

(a) $t=0.5$

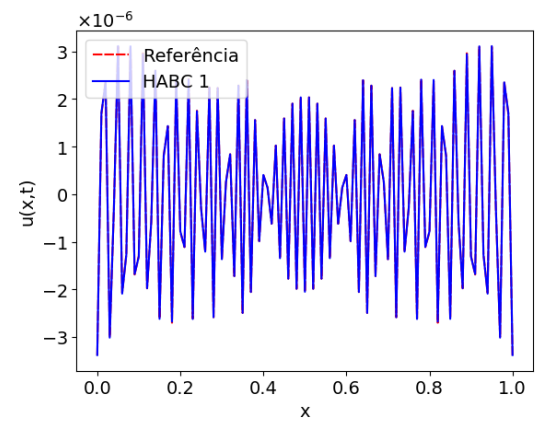

(b) $t=0.75$

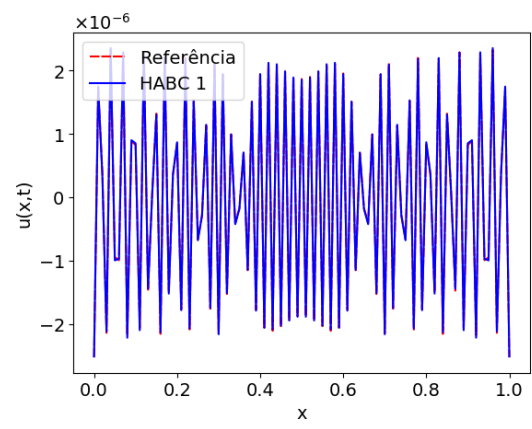

(c) $t=1$

Figura 3.6: Condição $H A B C 1$ aplicada ao primeiro exemplo unidimensional.

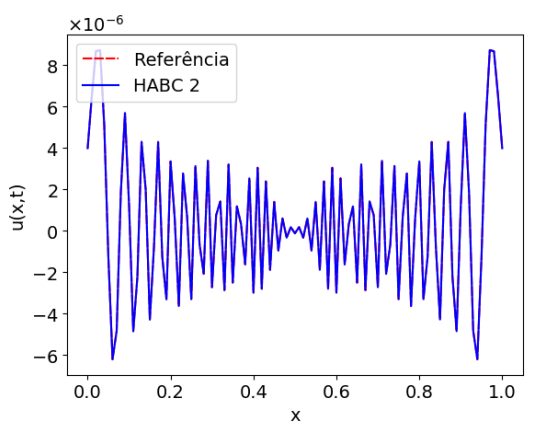

(a) $t=0.5$

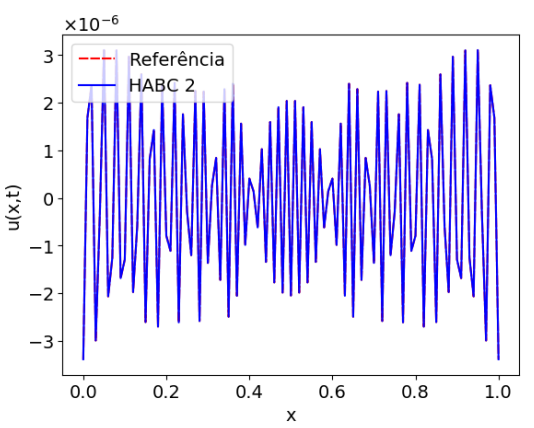

(b) $t=0.75$

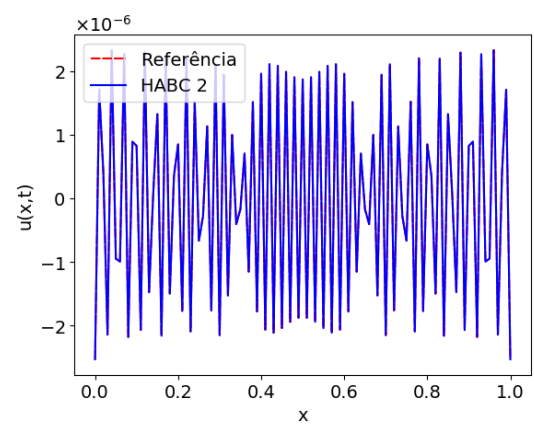

(c) $t=1$

Figura 3.7: Condição HABC 2, com $M=2$ e $\alpha=1.5+0.07(L-M)$, aplicada ao primeiro exemplo unidimensional.

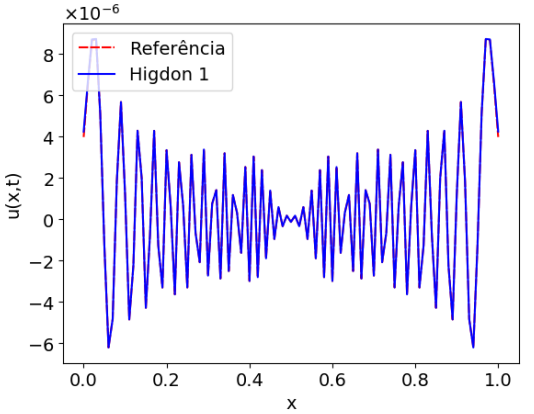

(a) $t=0.5$

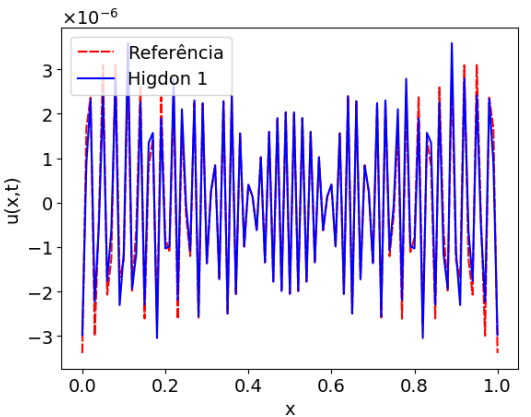

(b) $t=0.75$

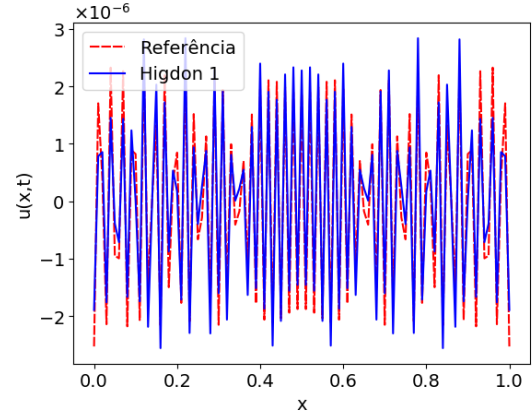

(c) $t=1$

Figura 3.8: Condição de Higdon de primeira ordem, com $\alpha=\frac{\pi}{8}, a=b=0.5$, aplicada ao primeiro exemplo unidimensional. 


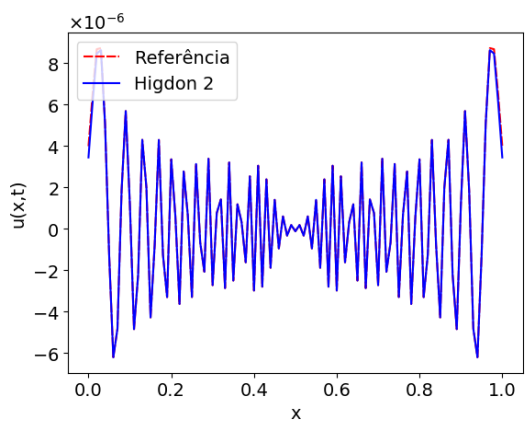

(a) $t=0.5$

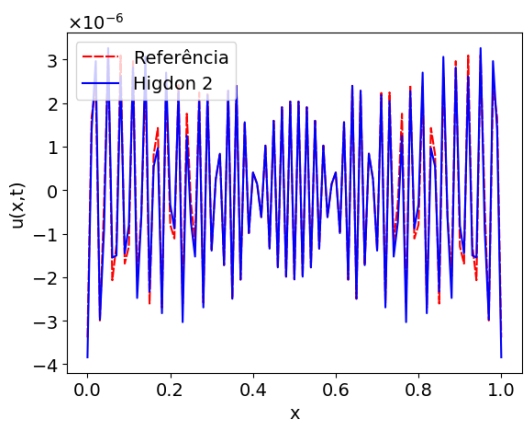

(b) $t=0.75$

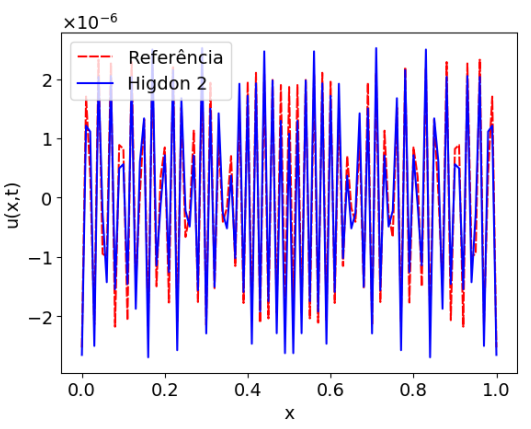

(c) $t=1$

Figura 3.9: Condição de Higdon de segunda ordem, com $\alpha_{1}=\frac{\pi}{4}, \alpha_{2}=\frac{\pi}{3}, a_{1}=a_{2}=b_{1}=b_{2}=0.5$, aplicada ao primeiro exemplo unidimensional.

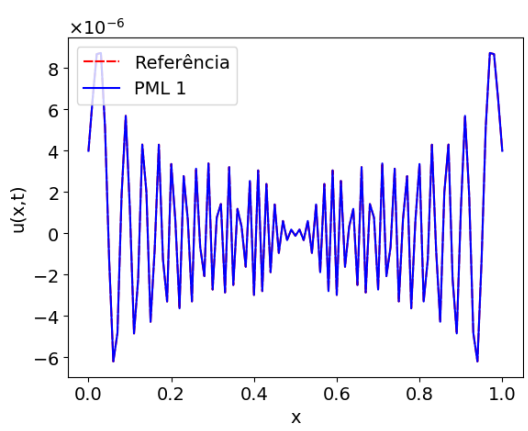

(a) $t=0.5$

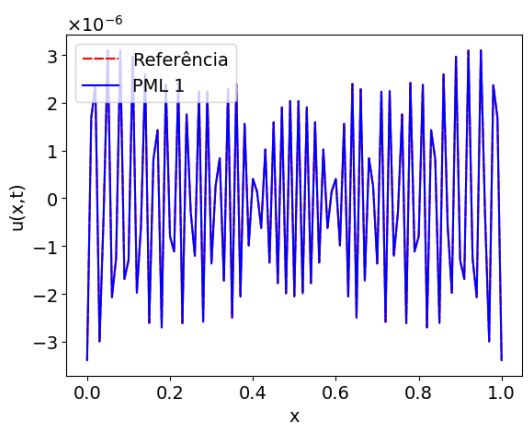

(b) $t=0.75$

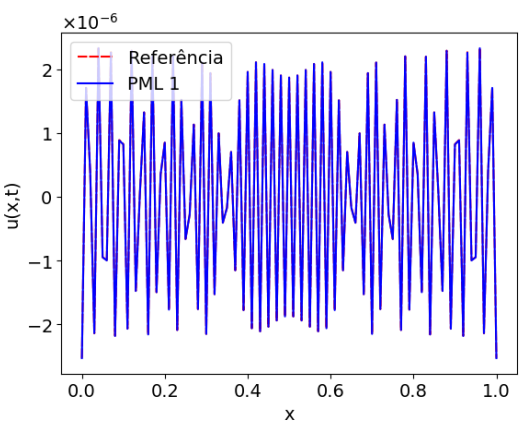

(c) $t=1$

Figura 3.10: Condição PML para o sistema de primeira ordem, com $R=10^{-3}$, aplicada ao primeiro exemplo unidimensional.

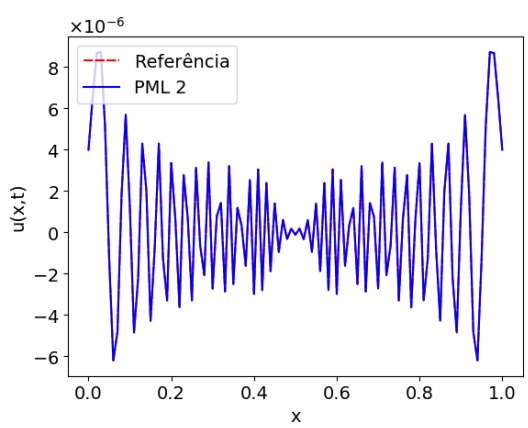

(a) $t=0.5$

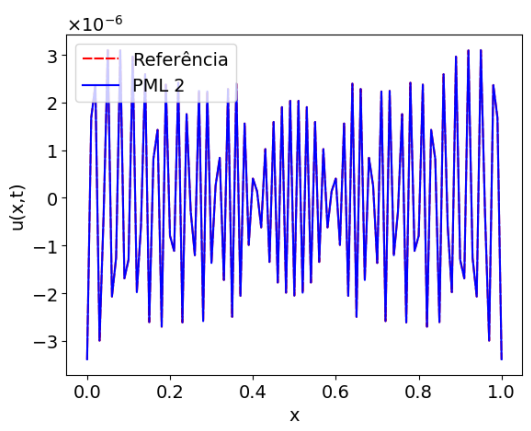

(b) $t=0.75$

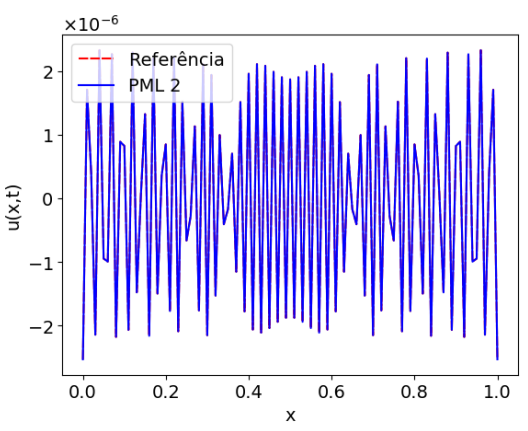

(c) $t=1$

Figura 3.11: Condição PML para a equação de segunda ordem, com $R=10^{-7}$, aplicada ao primeiro exemplo unidimensional. 


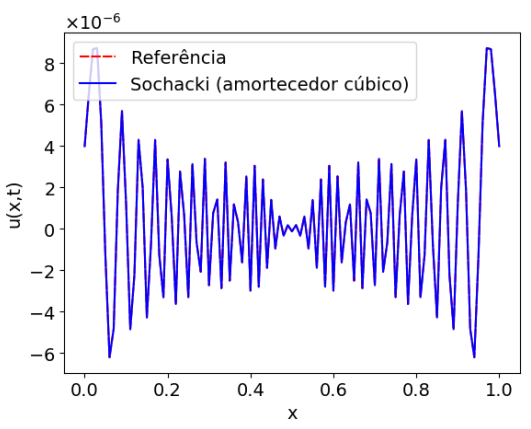

(a) $t=0.5$

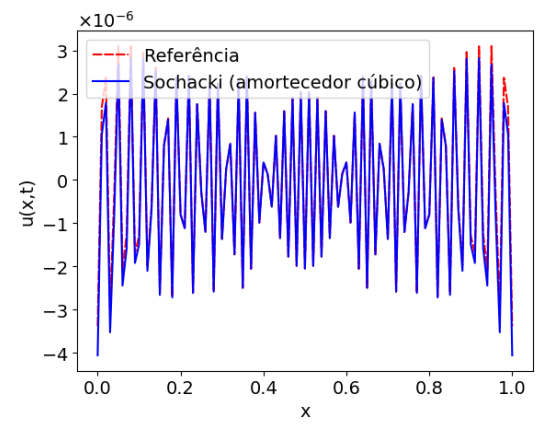

(b) $t=0.75$

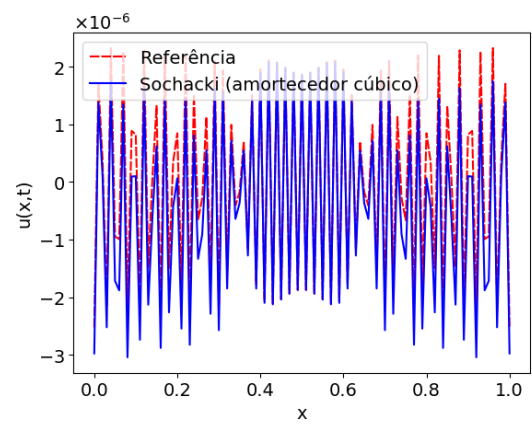

(c) $t=1$

Figura 3.12: Condição de Sochacki com amortecedor cúbico, com $S=10^{7.5}$, aplicada ao primeiro exemplo unidimensional.

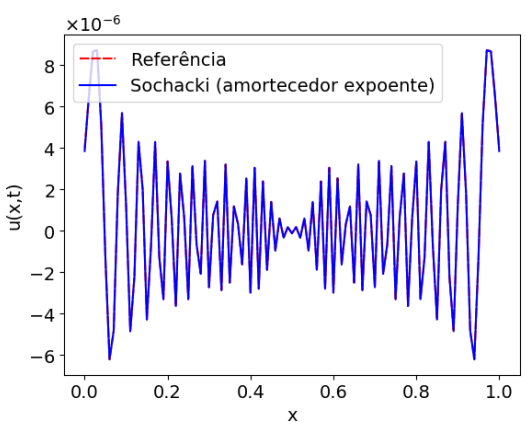

(a) $t=0.5$

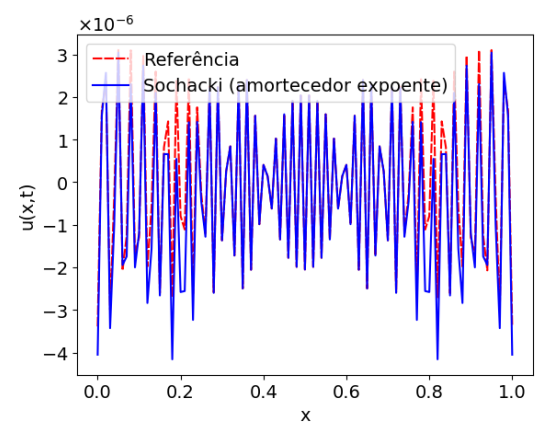

(b) $t=0.75$

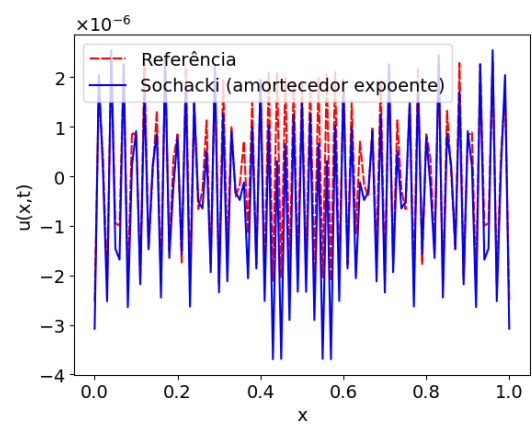

(c) $t=1$

Figura 3.13: Condição de Sochacki com amortecedor expoente, com $S=10$, aplicada ao primeiro exemplo unidimensional.

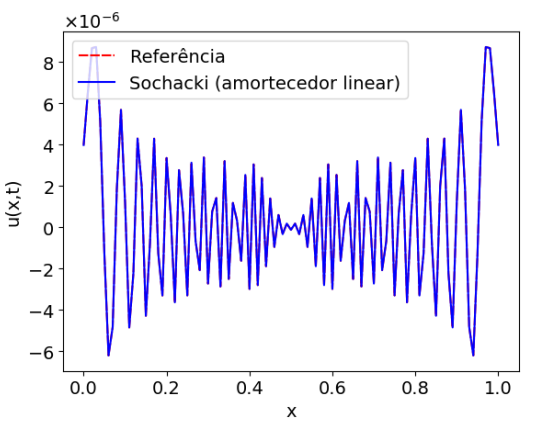

(a) $t=0.5$

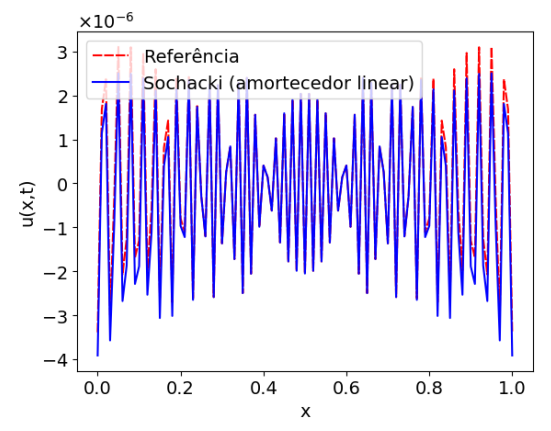

(b) $t=0.75$

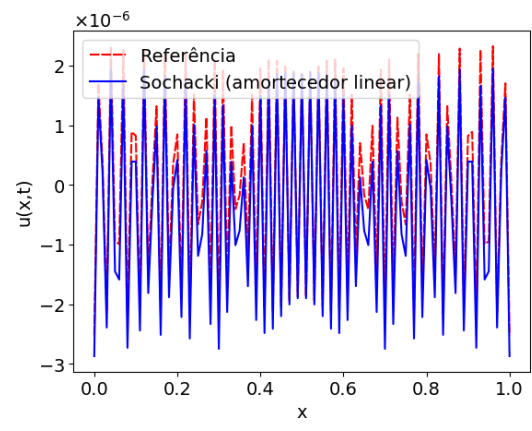

(c) $t=1$

Figura 3.14: Condição de Sochacki com amortecedor linear, com $S=10^{3.25}$, aplicada ao primeiro exemplo unidimensional.

Em termos de compatibilidade com a propagação da onda acústica em meios ilimitados, os resultados apresentados em 3.3a, 3.3b e 3.3c, obtidos a partir da aplicação da condição de Dirichlet, são inferiores aos fornecidos pelo uso das demais condições de contorno, devido ao fato de que essa condição não possui capacidade de absorção ou amortecimento das ondas incidentes.

Para a condição de Cerjan, conforme a figura 3.4a, as reflexões causadas pelo truncamento do domínio ainda não são visíveis para $t=0.5$, tendo em vista a extensão realizada no domínio a ser observado, necessária para a aplicação da condição de amortecimento. Já em $3.4 \mathrm{~b}$ e $3.4 \mathrm{c}$, a reflexão causada pelo truncamento do domínio pode ser percebida e, assim, vemos que a solução fornecida pela aplicação da condição de Cerjan preserva a forma da solução original para os valores de $t$ observados, no sentido de que os pontos de máximos e mínimos locais são mantidos, mas os 
valores obtidos para $u(x, t)$ não correspondem à solução esperada, tornando evidentes as limitações do método.

Como vimos, a condição A1 não conta com a extensão do domínio; no entanto, a solução obtida através da aplicação dessa condição ainda coincide com a solução original para $t=0.5$, conforme a figura 3.5a, ao contrário da condição de Dirichlet. Além disso, embora não preserve totalmente a forma da solução esperada, as reflexões causadas pelo truncamento do domínio nesse caso são menores em valor absoluto do que as observadas para a condição de Cerjan. Porém, é importante observar que, embora a condição A1 seja desenvolvida de modo a proporcionar absorção completa das ondas incidentes para o problema unidimensional contínuo, sua aplicação ao caso discreto pode não apresentar a mesma eficácia.

Para a HABC 1, utilizamos uma região de amortecimento na qual a solução é calculada como uma média ponderada entre o valor de $u\left(x_{i}, t_{n}\right)$ dado pela equação original e o valor fornecido pela condição de contorno A1, baseada na OWWE. É possível observar nas imagens 3.6a, 3.6b e 3.6c que o desempenho dessa condição de contorno é superior ao das condições analisadas anteriormente, com redução significativa da amplitude das reflexões em comparação com a condição A1 e o método de Cerjan e preservação da forma original da solução. A condição HABC 2, aplicada com o uso da condição A1, também apresenta eficácia considerável, com baixa reflexão das ondas incidentes no contorno.

Para a condição de Higdon de primeira ordem, podemos ver que as escolhas feitas para aplicação do método não resultaram em reflexões menores do que as resultantes da condição A1, e o mesmo vale para a condição de segunda ordem. Esses resultados refletem o fato de que a condição A1 para a equação unidimensional da onda acústica é desenvolvida visando a absorção completa das ondas incidentes, de modo que as condições de Higdon com $\alpha \neq 0$ não apresentam a mesma eficácia.

Conforme os resultados apresentados nas figuras 3.10a, 3.10b e 3.10c, a aplicação da PML para o sistema de primeira ordem ocasionou reflexões consideravelmente baixas, indicando que essa forma da PML forneceu absorção quase completa das ondas incidentes neste caso. Os resultados para a PML para a equação de segunda ordem são semelhantes aos verificados para o sistema de primeira ordem, de modo que ambos os métodos podem ser considerados dois dos mais favoráveis a este exemplo.

O uso da condição de Sochacki com amortecedor cúbico permitiu a preservação da forma da solução esperada, embora as amplitudes sejam alteradas. Apesar de apresentar desempenho inferior às condições HABC, PML e à condição A1, esse método fornece resultados superiores aos decorrentes do uso da condição de Cerjan, sendo ambos métodos de amortecimento. O mesmo ocorre com os amortecedores expoente e linear, que também são consideravelmente superiores à condição de Cerjan, mesmo apresentando discrepâncias em relação ao resultado esperado.

A seguir, apresentamos a tabela de erros obtidos com cada condição de contorno em relação ao tempo. 


\begin{tabular}{c|cccc|cccc}
\multirow{2}{*}{$t$} & \multicolumn{3}{|c|}{ Erro relativo na norma infinito } & \multicolumn{4}{c}{ Erro absoluto na norma 2} \\
& $t=0.5$ & $t=1$ & $t=1.5$ & $t=2$ & $t=0.5$ & $t=1$ & $t=1.5$ & $t=2$ \\
\hline Dirichlet & 0.238 & 3.399 & 3.356 & 6.106 & $3.28 \cdot 10^{-7}$ & $3.30 \cdot 10^{-6}$ & $3.19 \cdot 10^{-6}$ & $3.70 \cdot 10^{-6}$ \\
Cerjan & $8.92 \cdot 10^{-6}$ & 1.37 & 2.691 & 2.921 & $8.47 \cdot 10^{-12}$ & $1.37 \cdot 10^{-6}$ & $1.68 \cdot 10^{-6}$ & $1.43 \cdot 10^{-6}$ \\
A1 & 0.004 & 0.358 & 0.533 & 0.776 & $8.58 \cdot 10^{-9}$ & $3.76 \cdot 10^{-7}$ & $5.28 \cdot 10^{-7}$ & $6.09 \cdot 10^{-7}$ \\
HABC 1 & $8.81 \cdot 10^{-4}$ & 0.024 & 0.072 & 0.141 & $1.02 \cdot 10^{-9}$ & $2.07 \cdot 10^{-8}$ & $5.25 \cdot 10^{-8}$ & $9.13 \cdot 10^{-8}$ \\
HABC 2 & $1.71 \cdot 10^{-5}$ & 0.002 & 0.003 & 0.011 & $1.81 \cdot 10^{-11}$ & $1.75 \cdot 10^{-9}$ & $1.96 \cdot 10^{-9}$ & $4.45 \cdot 10^{-9}$ \\
Higdon 1 & 0.030 & 0.396 & 0.651 & 0.856 & $3.24 \cdot 10^{-8}$ & $4.69 \cdot 10^{-7}$ & $6.06 \cdot 10^{-7}$ & $6.63 \cdot 10^{-7}$ \\
Higdon 2 & 0.062 & 0.339 & 0.607 & 0.676 & $8.28 \cdot 10^{-8}$ & $3.95 \cdot 10^{-7}$ & $5.28 \cdot 10^{-7}$ & $5.42 \cdot 10^{-7}$ \\
PML 1 & $5.58 \cdot 10^{-6}$ & 0.003 & 0.004 & 0.014 & $6.63 \cdot 10^{-12}$ & $2.05 \cdot 10^{-9}$ & $2.79 \cdot 10^{-9}$ & $6.71 \cdot 10^{-9}$ \\
PML 2 & $1.30 \cdot 10^{-5}$ & 0.002 & 0.009 & 0.050 & $1.55 \cdot 10^{-11}$ & $1.19 \cdot 10^{-9}$ & $5.26 \cdot 10^{-9}$ & $2.39 \cdot 10^{-8}$ \\
S. Cúbico & $1.03 \cdot 10^{-5}$ & 0.393 & 0.622 & 0.174 & $1.00 \cdot 10^{-11}$ & $5.41 \cdot 10^{-7}$ & $6.93 \cdot 10^{-7}$ & $1.11 \cdot 10^{-7}$ \\
S. Expoente & 0.016 & 0.762 & 1.064 & 0.532 & $1.60 \cdot 10^{-8}$ & $6.31 \cdot 10^{-7}$ & $7.46 \cdot 10^{-7}$ & $3.95 \cdot 10^{-7}$ \\
S. Linear & $6.74 \cdot 10^{-4}$ & 0.260 & 0.437 & 0.126 & $6.71 \cdot 10^{-10}$ & $4.47 \cdot 10^{-7}$ & $5.88 \cdot 10^{-7}$ & $7.83 \cdot 10^{-8}$
\end{tabular}

Tabela 3.1: Erros para o primeiro teste unidimensional.

Como podemos ver, as HABCs e as PMLs apresentaram o melhor desempenho neste exemplo, enquanto a condição de Dirichlet forneceu o resultado menos satisfatório, seguida da condição de Cerjan. Observamos também que a condição de Higdon de segunda ordem resultou em erros maiores do que os obtidos para a condição de primeira ordem e que a PML para o sistema de primeira ordem forneceu resultados superiores aos verificados para a versão para a equação de segunda ordem. De modo geral, a HABC 2 forneceu resultados ligeiramente superiores aos obtidos para a PML 1, sendo ambos os melhores métodos para este exemplo. Na próxima seção, apresentamos alguns resultados acerca da sensibilidade de cada condição de contorno em relação ao número de CFL.

\subsubsection{Efetividade das condições de contorno em relação a $\lambda$}

A seguir, apresentamos a tabela de erros obtidos com cada condição de contorno para diferentes valores de $\lambda$, obtidos por meio de alteração no valor de $\Delta t$. 


\begin{tabular}{|c|c|c|c|c|c|c|c|c|c|}
\hline \multirow{2}{*}{ Método } & & \multicolumn{4}{|c|}{ Erro relativo na norma infinito } & \multicolumn{4}{|c|}{ Erro absoluto na norma 2} \\
\hline & & $\begin{array}{l}\lambda= \\
0.25\end{array}$ & $\lambda=0.5$ & $\lambda=0.8$ & $\lambda=1$ & $\lambda=0.25$ & $\lambda=0.5$ & $\lambda=0.8$ & $\lambda=1$ \\
\hline \multirow{3}{*}{ Dirichlet } & 1.2 & 4.08 & 4.05 & 4.63 & 2.54 & $2.85 \cdot 10^{-6}$ & $3.34 \cdot 10^{-6}$ & $3.99 \cdot 10^{-6}$ & $4.84 \cdot 10^{-5}$ \\
\hline & 1.6 & 5.25 & 6.06 & 6.66 & 2 & $2.93 \cdot 10^{-6}$ & $3.44 \cdot 10^{-6}$ & $3.43 \cdot 10^{-6}$ & $6 \cdot 10^{-5}$ \\
\hline & 2 & 4.95 & 6.11 & 6.58 & 2 & $3.24 \cdot 10^{-6}$ & $3.70 \cdot 10^{-6}$ & $4.36 \cdot 10^{-6}$ & $3.27 \cdot 10^{-5}$ \\
\hline \multirow{3}{*}{ Cerjan } & 1.2 & 3.15 & 1.57 & 2.66 & 1.85 & $1.87 \cdot 10^{-6}$ & $1.77 \cdot 10^{-6}$ & $2.23 \cdot 10^{-6}$ & $2.94 \cdot 10^{-5}$ \\
\hline & 1.6 & 2.04 & 2.21 & 3.27 & 2.85 & $1.52 \cdot 10^{-6}$ & $1.69 \cdot 10^{-6}$ & $2.37 \cdot 10^{-6}$ & $5.53 \cdot 10^{-5}$ \\
\hline & 2 & 2.09 & 2.92 & 1.84 & 2 & $1.26 \cdot 10^{-6}$ & $1.43 \cdot 10^{-6}$ & $1.70 \cdot 10^{-6}$ & $6.41 \cdot 10^{-5}$ \\
\hline \multirow{3}{*}{ A1 } & 1.2 & 0.41 & 0.41 & 0.42 & 0 & $4.53 \cdot 10^{-7}$ & $4.49 \cdot 10^{-7}$ & $5.02 \cdot 10^{-7}$ & 0 \\
\hline & 1.6 & 0.59 & 0.61 & 0.62 & 0 & $5.85 \cdot 10^{-7}$ & $5.97 \cdot 10^{-7}$ & $6.52 \cdot 10^{-7}$ & 0 \\
\hline & 2 & 0.78 & 0.78 & 0.79 & 0 & $5.69 \cdot 10^{-7}$ & $6.09 \cdot 10^{-7}$ & $7.02 \cdot 10^{-7}$ & 0 \\
\hline \multirow{3}{*}{ HABC 1} & 1.2 & 0.07 & 0.04 & 0.04 & 0 & $6.20 \cdot 10^{-8}$ & $3.21 \cdot 10^{-8}$ & $3.14 \cdot 10^{-8}$ & 0 \\
\hline & 1.6 & 0.14 & 0.09 & & 0 & $9.97 \cdot 10^{-8}$ & $6.28 \cdot 10^{-8}$ & $7.62 \cdot 10^{-8}$ & 0 \\
\hline & 2 & 0.20 & 0.14 & 0.16 & 0 & $1.43 \cdot 10^{-7}$ & $9.13 \cdot 10^{-8}$ & $1.16 \cdot 10^{-7}$ & 0 \\
\hline \multirow{3}{*}{ HABC 2} & 1.2 & $4 \cdot 10^{-3}$ & $3 \cdot 10^{-3}$ & $1 \cdot 10^{-3}$ & 0 & $3.02 \cdot 10^{-9}$ & $1.74 \cdot 10^{-9}$ & $1.00 \cdot 10^{-9}$ & 0 \\
\hline & 1.6 & $9 \cdot 10^{-3}$ & $4 \cdot 10^{-3}$ & $6 \cdot 10^{-3}$ & 0 & $4.11 \cdot 10^{-9}$ & $2.13 \cdot 10^{-9}$ & $3.32 \cdot 10^{-9}$ & 0 \\
\hline & 2 & 0.02 & 0.01 & 0.02 & 0 & $8.34 \cdot 10^{-9}$ & $4.45 \cdot 10^{-9}$ & $1.07 \cdot 10^{-8}$ & 0 \\
\hline \multirow{3}{*}{ Higdon 1} & 1.2 & 0.44 & 0.44 & 0.45 & 0.10 & $5.42 \cdot 10^{-7}$ & $5.27 \cdot 10^{-7}$ & $5.93 \cdot 10^{-7}$ & $1.94 \cdot 10^{-6}$ \\
\hline & 1.6 & 0.70 & 0.73 & 0.73 & 0.08 & $6.62 \cdot 10^{-7}$ & $6.78 \cdot 10^{-7}$ & $7.40 \cdot 10^{-7}$ & $2.56 \cdot 10^{-6}$ \\
\hline & 2 & 0.88 & 0.86 & 0.87 & 0.08 & $6.20 \cdot 10^{-8}$ & $6.63 \cdot 10^{-7}$ & $7.64 \cdot 10^{-7}$ & $2.52 \cdot 10^{-6}$ \\
\hline \multirow{3}{*}{ Higdon 2} & 1.2 & 0.35 & & 0.42 & 0.14 & $4.10 \cdot 10^{-7}$ & $4.64 \cdot 10^{-7}$ & $5.23 \cdot 10^{-7}$ & $2.84 \cdot 10^{-6}$ \\
\hline & 1.6 & 0.55 & 0.58 & 0.59 & 0.11 & $4.83 \cdot 10^{-7}$ & $4.96 \cdot 10^{-7}$ & $6.06 \cdot 10^{-7}$ & $3.69 \cdot 10^{-6}$ \\
\hline & 2 & 0.75 & 0.68 & 0.66 & 0.11 & $5.48 \cdot 10^{-7}$ & $5.42 \cdot 10^{-7}$ & $5.71 \cdot 10^{-7}$ & $3.60 \cdot 10^{-6}$ \\
\hline \multirow{3}{*}{ PML 1} & 1.2 & $3 \cdot 10^{-3}$ & $3 \cdot 10^{-3}$ & $4 \cdot 10^{-3}$ & 0.85 & $2.01 \cdot 10^{-9}$ & $2.28 \cdot 10^{-9}$ & $3.84 \cdot 10^{-9}$ & $2.30 \cdot 10^{-5}$ \\
\hline & 1.6 & $3 \cdot 10^{-3}$ & $5 \cdot 10^{-3}$ & 0.02 & 1.61 & $2.73 \cdot 10^{-9}$ & $3.54 \cdot 10^{-9}$ & $1.13 \cdot 10^{-8}$ & $4.69 \cdot 10^{-5}$ \\
\hline & 2 & 0.01 & 0.01 & 0.05 & 1.87 & $4.41 \cdot 10^{-9}$ & $6.71 \cdot 10^{-9}$ & $2.91 \cdot 10^{-8}$ & $5.72 \cdot 10^{-5}$ \\
\hline \multirow{3}{*}{ PML 2} & 1.2 & $3 \cdot 10^{-3}$ & $4 \cdot 10^{-3}$ & 0.01 & 0.93 & $1.65 \cdot 10^{-9}$ & $2.35 \cdot 10^{-9}$ & $7.09 \cdot 10^{-9}$ & $2.62 \cdot 10^{-5}$ \\
\hline & 1.6 & $9 \cdot 10^{-3}$ & 0.02 & 0.06 & 1.65 & $4.88 \cdot 10^{-9}$ & $8.18 \cdot 10^{-9}$ & $3.68 \cdot 10^{-8}$ & $4.93 \cdot 10^{-5}$ \\
\hline & 2 & 0.04 & 005 & 0.14 & 1.8 & $1.55 \cdot 10^{-7}$ & $2.39 \cdot 10^{-8}$ & $8.33 \cdot 10^{-8}$ & $5.35 \cdot 10^{-5}$ \\
\hline \multirow{3}{*}{ S. Cúbico } & 1.2 & 0.40 & & & 0.9 & -7 & $6.13 \cdot 10^{-7}$ & $6.62 \cdot 10^{-7}$ & $2.19 \cdot 10^{-5}$ \\
\hline & 1.6 & 0.55 & & & 1.77 & $6.08 \cdot 10^{-7}$ & $6.26 \cdot 10^{-7}$ & $6.76 \cdot 10^{-7}$ & $4.64 \cdot 10^{-5}$ \\
\hline & 2 & 0.17 & 0.17 & 0.20 & 1.98 & $1.06 \cdot 10^{-7}$ & $1.11 \cdot 10^{-7}$ & $1.28 \cdot 10^{-7}$ & $6.22 \cdot 10^{-5}$ \\
\hline \multirow{3}{*}{ S. Expoente } & 1 & 0.91 & 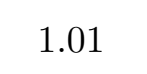 & 1.08 & 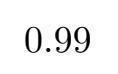 & $6.99 \cdot 10^{-7}$ & $7.24 \cdot 10^{-7}$ & $8.16 \cdot 10^{-7}$ & $2.56 \cdot 10^{-5}$ \\
\hline & 1.6 & 0.91 & & & 1 & $4.68 \cdot 10^{-7}$ & $5.12 \cdot 10^{-7}$ & $6.04 \cdot 10^{-7}$ & $4.66 \cdot 10^{-5}$ \\
\hline & 2 & 0.53 & 0.53 & 0.64 & 1.80 & $3.69 \cdot 10^{-7}$ & $3.90 \cdot 10^{-7}$ & $4.83 \cdot 10^{-7}$ & $5.06 \cdot 10^{-5}$ \\
\hline \multirow{3}{*}{ S. Linear } & 1.2 & 0.56 & 0.57 & 0.56 & 0.87 & $5.92 \cdot 10^{-7}$ & $6.10 \cdot 10^{-7}$ & $6.56 \cdot 10^{-7}$ & $2.22 \cdot 10^{-5}$ \\
\hline & 1.6 & 0.40 & 0.39 & 0.41 & 1.63 & $5.22 \cdot 10^{-7}$ & $5.36 \cdot 10^{-7}$ & $5.78 \cdot 10^{-7}$ & $4.62 \cdot 10^{-5}$ \\
\hline & 2 & 0.10 & 0.13 & 0.12 & 1.91 & $7.48 \cdot 10^{-8}$ & $7.83 \cdot 10^{-8}$ & $9.75 \cdot 10^{-8}$ & $5.81 \cdot 10^{-5}$ \\
\hline
\end{tabular}

Tabela 3.2: Erro para as condições de contorno com diversos valores de $\lambda$.

Os valores referentes à condição de contorno proposta por Cerjan mostram que esse método é ligeiramente sensível ao valor de $\lambda$ e os erros obtidos para $\lambda=0.8$ são mais altos do que para 0.25 e 0.5 , embora mais baixos do que para $\lambda=1$. A condição A1, por sua vez, resulta em erros bastante próximos para $\lambda=0.25,0.5$ e 0.8 . Observamos, ainda, que a reflexão é nula para $\lambda=1$. Um comportamento semelhante é verificado para as HABCs, que também apresentam reflexão nula para $\lambda=1$, embora as discrepâncias sejam mais significativas entre os demais valores de $\lambda$. Isso indica que as reflexões verificadas para as outras constantes de CFL se devem principalmente à diferença entre as velocidades de propagação original e numérica.

A aplicação da condição de Dirichlet resulta em erros bastante próximos para valores distintos 
de $\lambda$, assim como as condições de Higdon de primeira e segunda ordens. Para os métodos de Higdon, é importante destacar que os melhores resultados se verificam com $\lambda=1$, acompanhando o comportamento da condição A1 e das HABCs.

As duas PMLs apresentaram comportamentos semelhantes entre si, com erros consideravelmente distintos para os valores de $\lambda$ diversos. Para $\lambda=1$, a eficácia dos métodos é comprometida, tornandoos inferiores a condição A1, às HABCs e às condições de Higdon, por exemplo. As condições de Sochacki com os amortecedores cúbico, expoente e linear não sofreram grandes impactos com a alteração da constante de CFL, apresentando diferenças significativas de eficácia apenas para $\lambda=1$.

Tendo em vista os resultados obtidos, concluímos que a constante de CFL afetou significativamente o uso de algumas condições de contorno, de modo que a escolha do valor de $\lambda$ pode ser utilizada para aumentar a eficácia de tais métodos.

\subsection{Equação da onda unidimensional - Exemplo 2}

Neste exemplo, avaliamos os efeitos da aplicação das condições de contorno abordadas anteriormente à resolução numérica da seguinte equação:

$$
\begin{aligned}
u_{t t}-u_{x x} & =0, x \in[0,1] \\
u(x, 0) & =e^{-0.5\left(\frac{x-0.5}{0.125}\right)^{2}} \\
u_{t}(x, 0) & =0 .
\end{aligned}
$$

A representação gráfica da condição inicial $u(x, 0)$ é a seguinte:

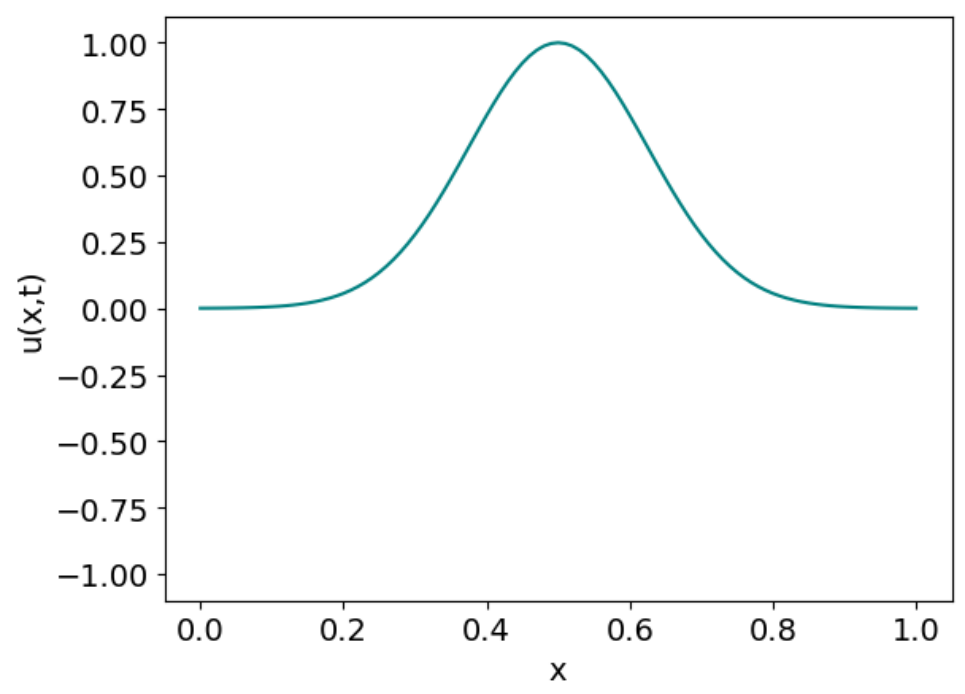

Figura 3.15: Condição inicial para o segundo exemplo unidimensional.

Nesse teste, a condição inicial $u(x, 0)$ é não nula, o termo de fonte é $f(x, t) \equiv 0$ e os efeitos do truncamento do domínio podem ser percebidos desde o início. As soluções apresentadas a seguir são obtidas com os seguintes valores:

- Tempo total de simulação: $T=1.5$;

- Malha de 100 pontos no espaço;

- $\Delta x=0.01, \Delta t=0.005, \lambda=c \frac{\Delta t}{\Delta x}=0.5$.

Nas figuras a seguir, apresentamos o comportamento da solução de referência: 


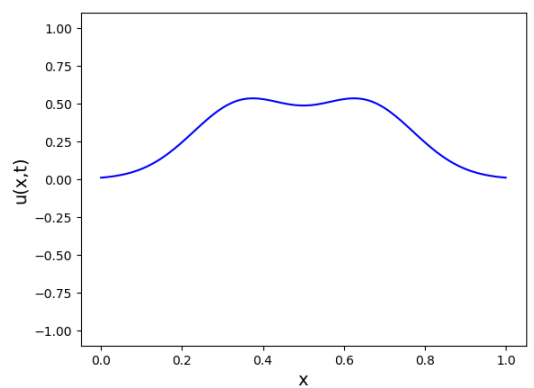

(a) $t=0.15$

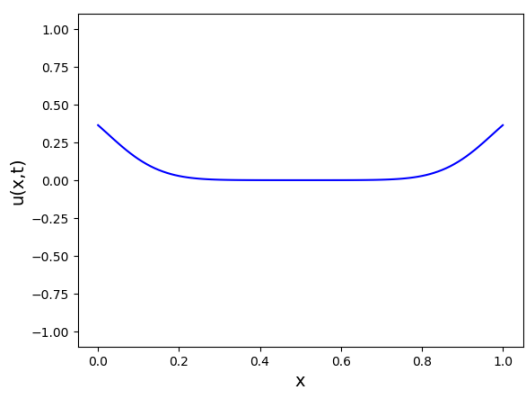

(d) $t=0.6$

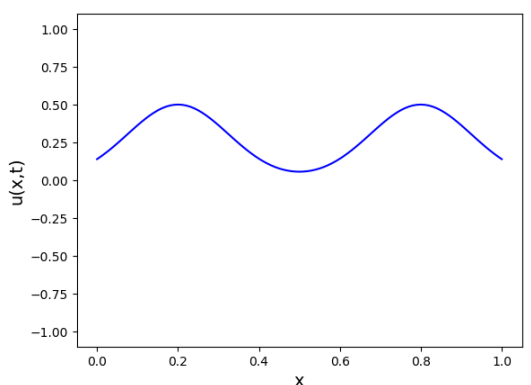

(b) $t=0.3$

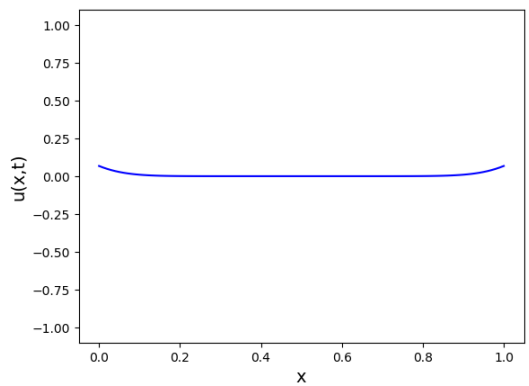

(e) $t=0.75$

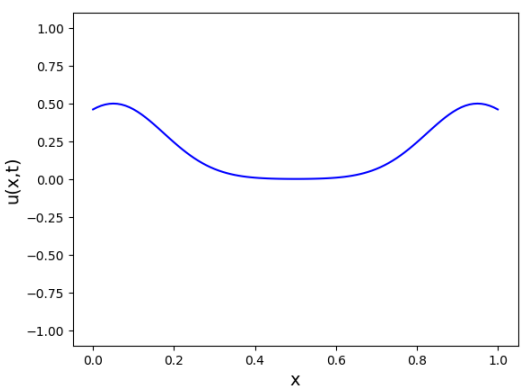

(c) $t=0.45$

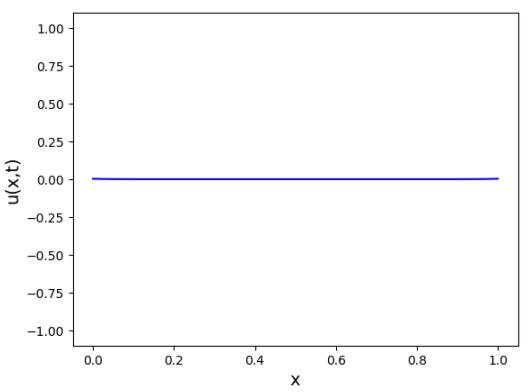

(f) $t=0.9$

Figura 3.16: Solução de referência no decorrer do tempo para o segundo exemplo com a equação da onda unidimensional.

Os resultados apresentados na primeira seção são obtidos com $\Delta x=0.01 \mathrm{e}$, na segunda seção, verificamos a eficácia de cada método em relação ao refinamento da malha, mantendo fixo o valor de $\lambda$ e utilizando $\Delta x=0.005$ e $\Delta x=0.0025$. A solução esperada, tomada como referência para as análises, é novamente obtida por meio da resolução da equação em um domínio suficientemente estendido, de modo que a solução no domínio de interesse não seja afetada pelas reflexões resultantes do truncamento do domínio. As medidas de erro adotadas nesse teste são

$$
\left\|e^{n}\right\|_{\infty}=\sup _{i}\left\{\left|u_{i}^{*^{n}}-u_{i}^{n}\right|\right\},\left\|e^{n}\right\|_{2}=\left(\frac{\sum_{i=0}^{N}\left|u_{i}^{*^{n}}-u_{i}^{n}\right|^{2}}{N}\right)^{\frac{1}{2}} .
$$

Os parâmetros aplicados a esse exemplo são:

- $L=20$, de modo que as camadas de amortecimento têm espessura $\delta=0.2$, para $\Delta x=0.01$, $\delta=0.1$, para $\Delta x=0.005$ e $\delta=0.05$ para $\Delta x=0.0025$

- Cerjan: $\tilde{\lambda}=0.0001$;

- HABC 2: $M=2, \alpha=1.5+0.07(L-M)$;

- Condição de Higdon de primeira ordem: $\alpha=\frac{\pi}{8}, a=b=0.5$;

- Condição de Higdon de segunda ordem: $\alpha_{1}=\frac{\pi}{4}, \alpha_{2}=\frac{\pi}{3}, a_{1}=a_{2}=b_{1}=b_{2}=0.5$;

- PML para o sistema de primeira ordem: $R=10^{-4}$,

$$
d(x)=\left\{\begin{array}{l}
\frac{3}{40 \Delta x} \ln \left(\frac{1}{0.0001}\right)\left(\frac{x}{20 \Delta x}\right)^{2}, \text { se } x<0 \\
\frac{3}{40 \Delta x} \ln \left(\frac{1}{0.0001}\right)\left(\frac{x-1}{20 \Delta x}\right)^{2}, \text { se } x>1
\end{array}\right.
$$


- PML para a equação de segunda ordem: $R=10^{-7}$,

$$
d(x)=\left\{\begin{array}{l}
\frac{3}{40 \Delta x} \ln \left(\frac{1}{10^{-7}}\right)\left(\frac{x}{20 \Delta x}\right)^{2}, \text { se } x<0 \\
\frac{3}{40 \Delta x} \ln \left(\frac{1}{10^{-7}}\right)\left(\frac{x-1}{20 \Delta x}\right)^{2}, \text { se } x>1
\end{array}\right.
$$

- Condição de Sochacki com amortecedor cúbico: $S=10^{7.75}$;

- Condição de Sochacki com amortecedor expoente: $S=10^{0.75}$;

- Condição de Sochacki com amortecedor linear: $S=10^{3.4}$.

\subsubsection{Resultados para $\Delta x=0.01$ e $\Delta t=0.005$}

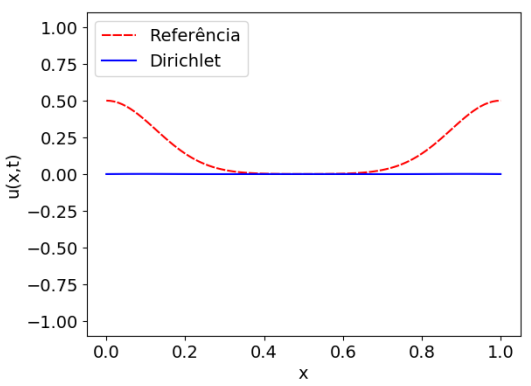

(a) $t=0.5$

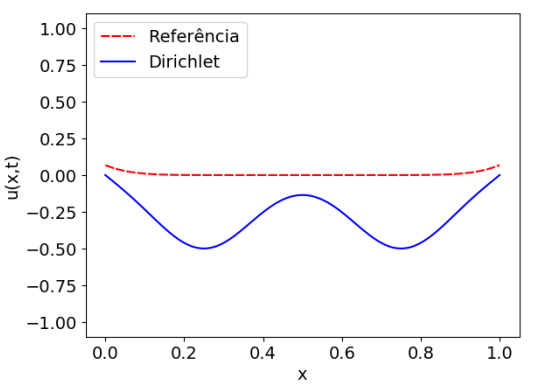

(b) $t=0.75$

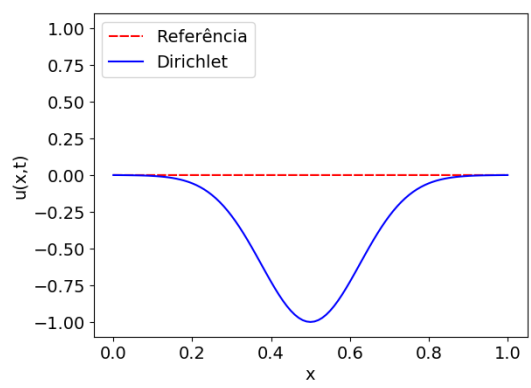

(c) $t=1$

Figura 3.17: Condição de Dirichlet $u=0$ aplicada ao segundo exemplo unidimensional.

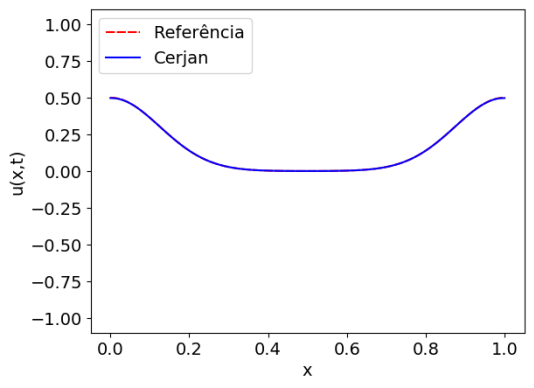

(a) $t=0.5$

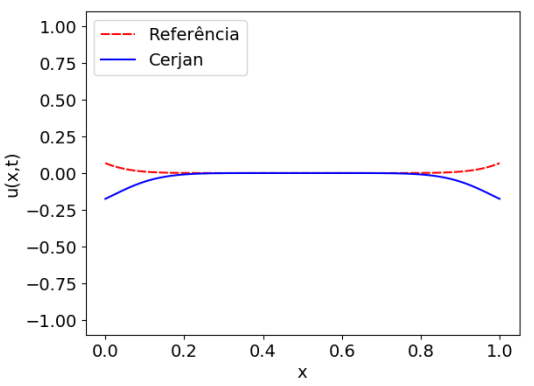

(b) $t=0.75$

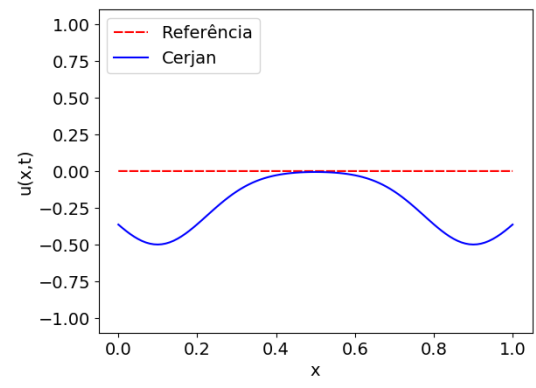

(c) $t=1$

Figura 3.18: Condição de Cerjan, com $\tilde{\lambda}=0.0001$, aplicada ao segundo exemplo unidimensional.

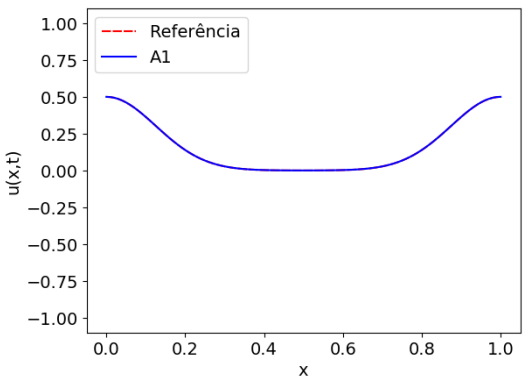

(a) $t=0.5$

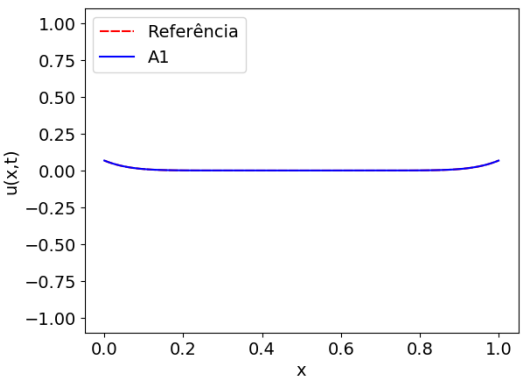

(b) $t=0.75$

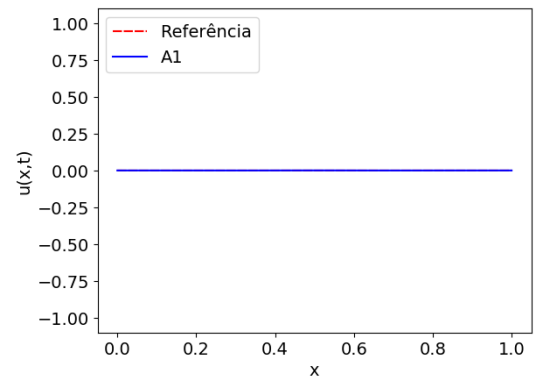

(c) $t=1$

Figura 3.19: Condição A1 aplicada ao segundo exemplo unidimensional. 


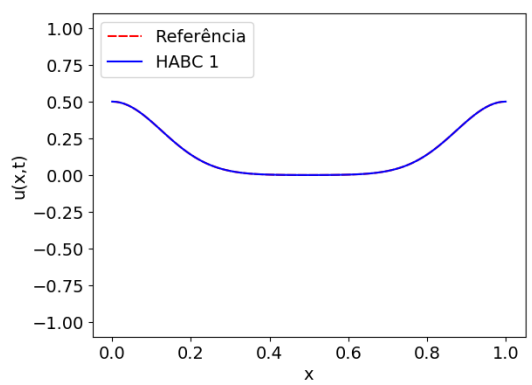

(a) $t=0.5$

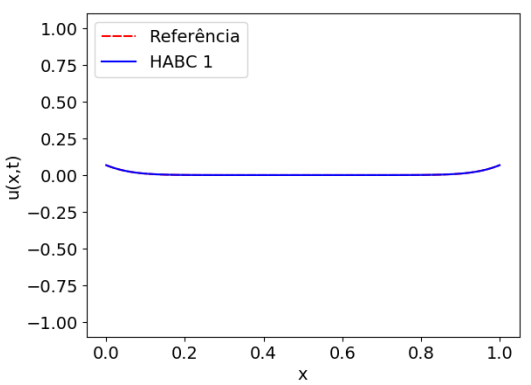

(b) $t=0.75$

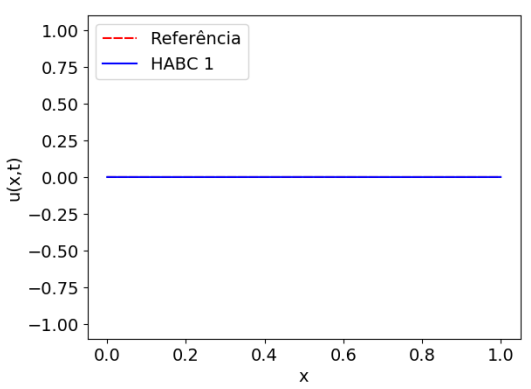

(c) $t=1$

Figura 3.20: Condição HABC 1 aplicada ao segundo exemplo unidimensional.

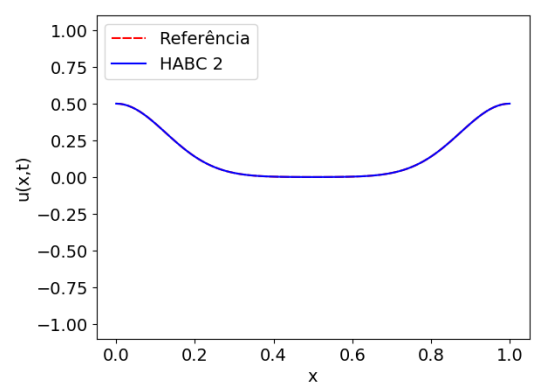

(a) $t=0.5$

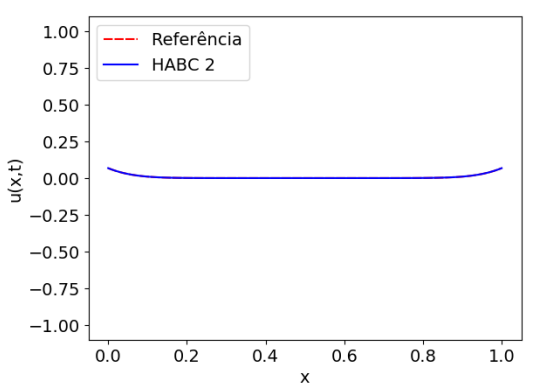

(b) $t=0.75$

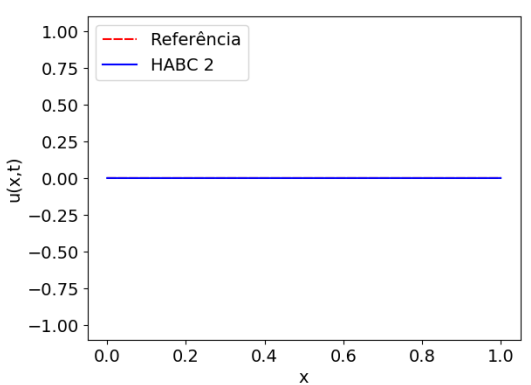

(c) $t=1$

Figura 3.21: Condição $H A B C$ 2, com $M=2$ e $\alpha=1.5+0.07(L-M)$, aplicada ao segundo exemplo unidimensional.

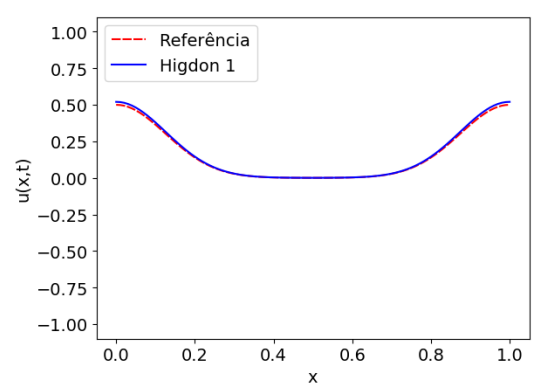

(a) $t=0.5$

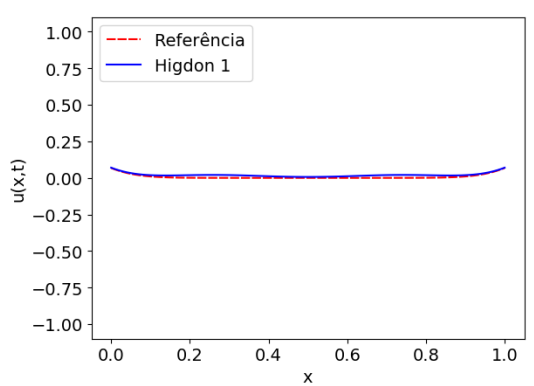

(b) $t=0.75$

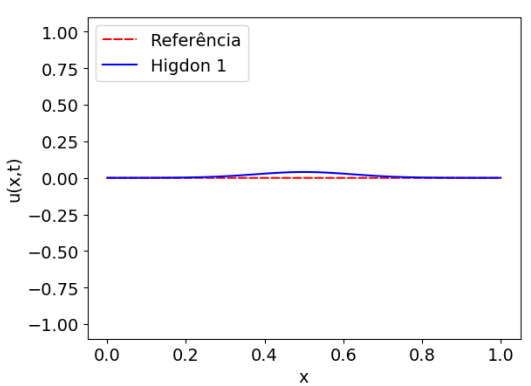

(c) $t=1$

Figura 3.22: Condição de Higdon de primeira ordem, com $\alpha=\frac{\pi}{8}, a=b=0.5$, aplicada ao segundo exemplo unidimensional.

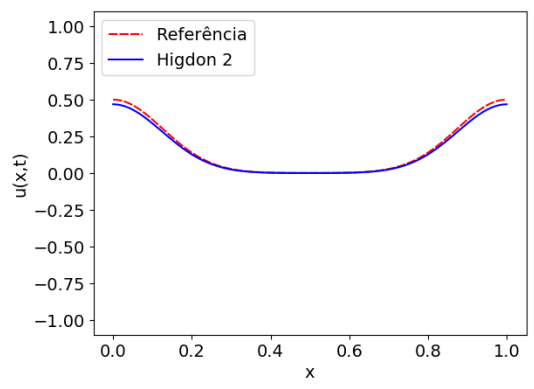

(a) $t=0.5$

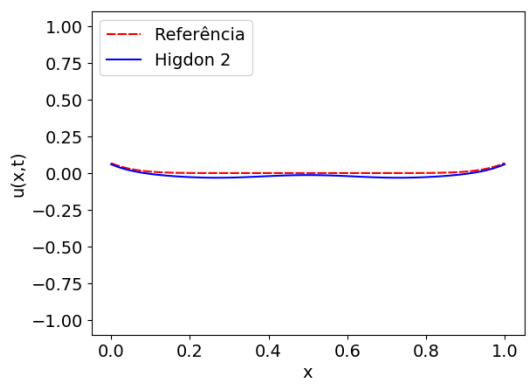

(b) $t=0.75$

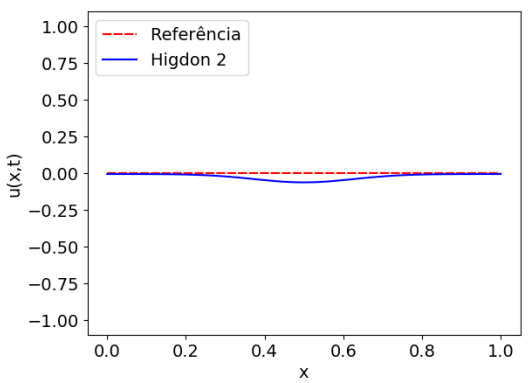

(c) $t=1$

Figura 3.23: Condição de Higdon de segunda ordem, com $\alpha_{1}=\frac{\pi}{4}, \alpha_{2}=\frac{\pi}{3}, a_{1}=a_{2}=b_{1}=b_{2}=0.5$, aplicada ao segundo exemplo unidimensional. 


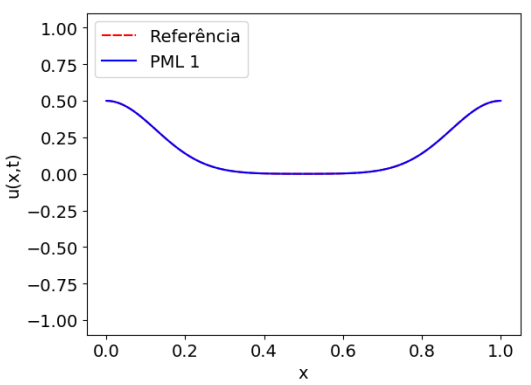

(a) $t=0.5$

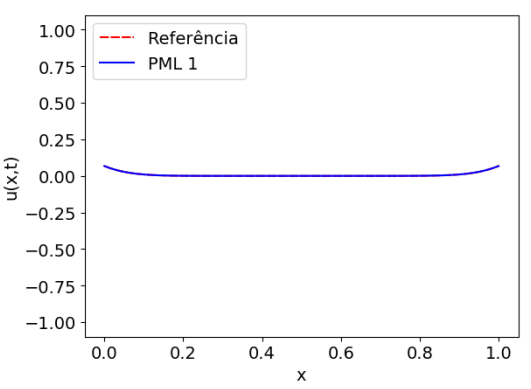

(b) $t=0.75$

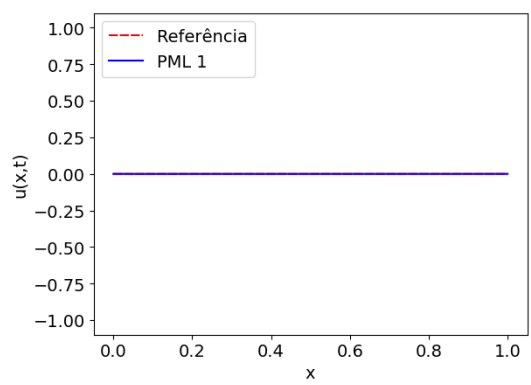

(c) $t=1$

Figura 3.24: Condição PML para o sistema de primeira ordem, com $R=10^{-4}$, aplicada ao segundo exemplo unidimensional.

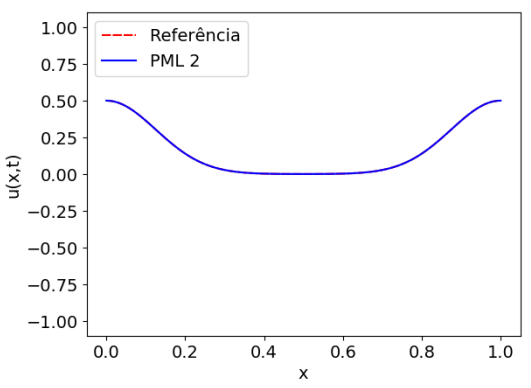

(a) $t=0.5$

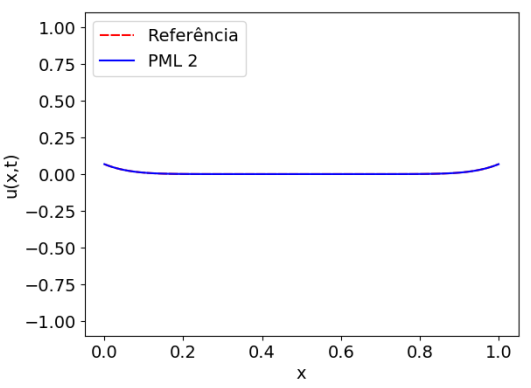

(b) $t=0.75$

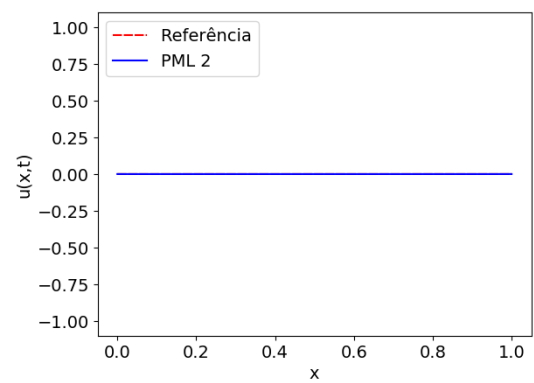

(c) $t=1$

Figura 3.25: Condição PML para a equação de segunda ordem, com $R=10^{-7}$, aplicada ao segundo exemplo unidimensional.

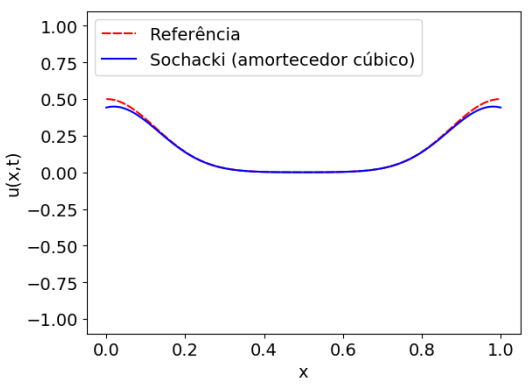

(a) $t=0.5$

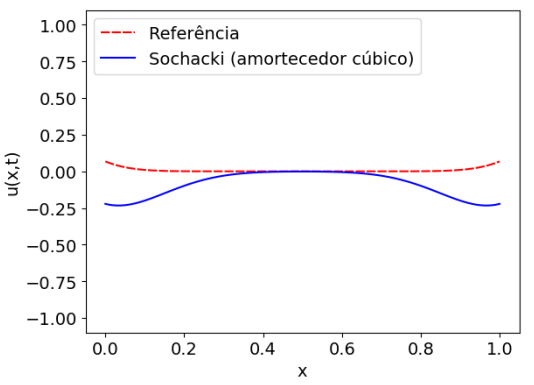

(b) $t=0.75$

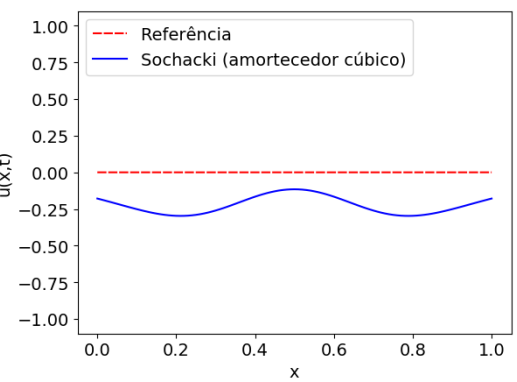

(c) $t=1$

Figura 3.26: Condição de Sochacki com amortecedor cúbico, com $S=10^{7.75}$, aplicada ao segundo exemplo unidimensional.

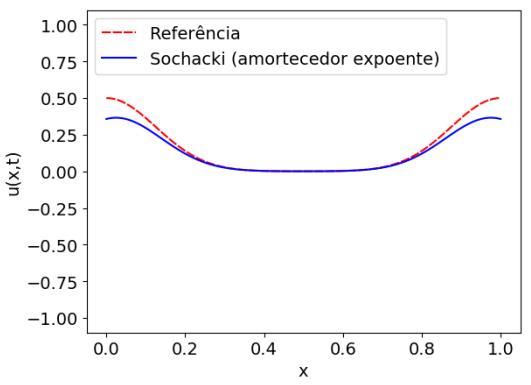

(a) $t=0.5$

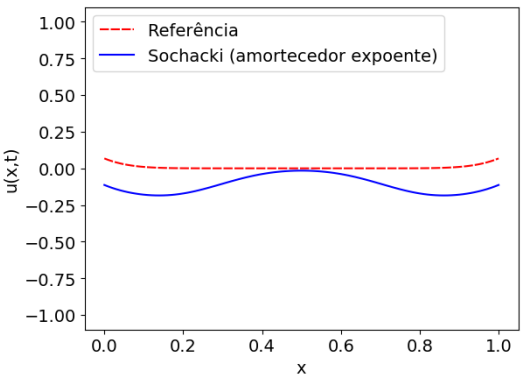

(b) $t=0.75$

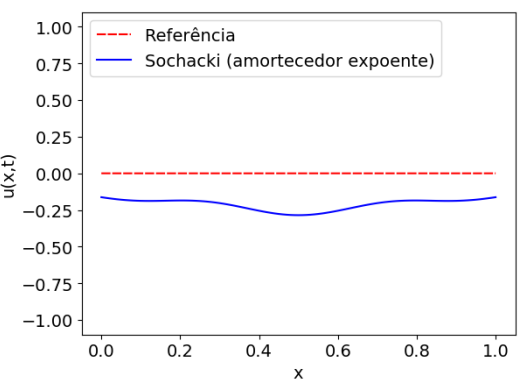

(c) $t=1$

Figura 3.27: Condição de Sochacki com amortecedor expoente, com $S=10^{0.75}$, aplicada ao segundo exemplo unidimensional. 


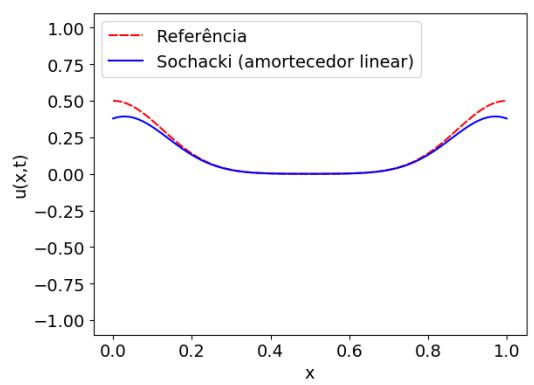

(a) $t=0.5$

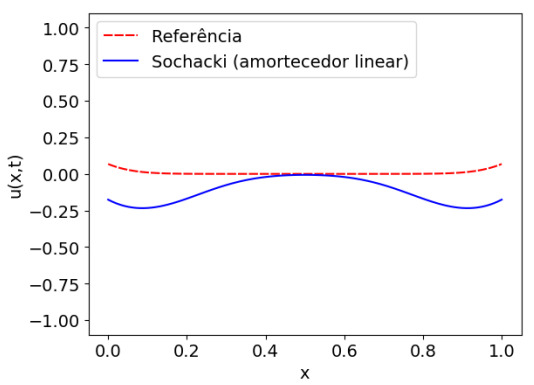

(b) $t=0.75$

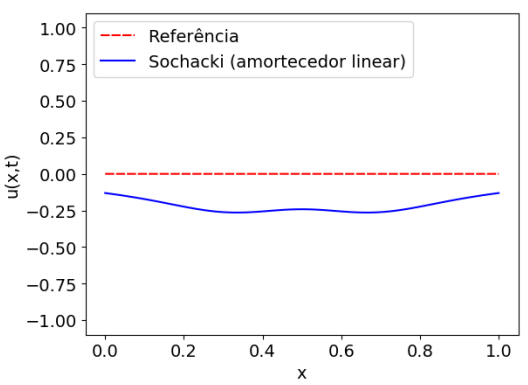

(c) $t=1$

Figura 3.28: Condição de Sochacki com amortecedor linear, com $S=10^{3.4}$, aplicada ao segundo exemplo unidimensional.

Neste exemplo, é possível ver de forma mais clara os efeitos da aplicação da condição de Dirichlet à resolução numérica da equação da onda. Tendo fixas as duas extremidades da solução, a onda se propaga somente no interior do domínio analisado, de modo que a mesma é completamente refletida, conforme os resultados teóricos apresentados no capítulo anterior.

Para o método proposto por Cerjan, podemos ver que as reflexões resultantes da aplicação dessa condição de contorno são menores que as apresentadas para a condição de Dirichlet em $t=0.6$. Esse comportamento se deve, de certa forma, à extensão do domínio necessária para a aplicação da condição de Cerjan, que não é realizada na condição de Dirichlet. Para $t=0.75$, as reflexões são visivelmente maiores e a forma da solução não é preservada. O mesmo ocorre para $t=1$, de modo que a condição de Cerjan não proporciona os melhores resultados entre os métodos abordados.

A aplicação da condição A1, por sua vez, apresenta resultados próximos à solução esperada para todos os valores de $t$ observados, e o mesmo ocorre para as HABCs e as PMLs. Já para as condições de Higdon obtemos novamente resultados inferiores aos obtidos através da aplicação da condição A1 (e, consequentemente, das HABCs e das PMLs). É possível observar, inclusive, que a condição de segunda ordem apresenta resultado inferior ao fornecido pela condição de primeira ordem.

A aplicação da condição de Sochacki, por sua vez, apresenta resultados semelhantes para os três amortecedores utilizados. O uso dessa condição de contorno resulta em reflexões perceptivelmente maiores do que as verificadas para as HABCs, as PMLs, as condições de Higdon e a A1, e a solução obtida difere consideravelmente do resultado da aplicação da condição de Cerjan, embora ambas sejam condições de amortecimento.

A comparação entre as figuras referentes à aplicação de cada condição não é suficiente para que se possa inferir qual método possui o melhor desempenho, de modo que é necessária uma verificação mais detalhada nesse caso. A seguinte tabela de erros apresenta dados que possibilitam tal análise:

\begin{tabular}{c|ccc|ccc}
\multirow{2}{*}{ Método } & \multicolumn{3}{|c|}{ Erro absoluto na norma infinito } & \multicolumn{3}{c}{ Erro absoluto na norma 2} \\
& $t=0.5$ & $t=0.75$ & $t=1$ & $t=0.5$ & $t=0.75$ & $t=1$ \\
\hline Dirichlet & 0.5 & 0.5 & 1 & 0.2351 & 0.3355 & 0.4706 \\
Cerjan & 0.0030 & 0.2429 & 0.5 & 0.0006 & 0.0706 & 0.3105 \\
A1 & 0.0003 & 0.0005 & 0.0007 & 0.0002 & 0.0003 & 0.0004 \\
HABC 1 & 0.0002 & 0.0002 & 0.0003 & $6.3326 \cdot 10^{-5}$ & 0.0001 & 0.0001 \\
HABC 2 & $3.739 \cdot 10^{-5}$ & 0.0001 & 0.0001 & $1.8720 \cdot 10^{-5}$ & $7.3037 \cdot 10^{-5}$ & $6.3905 \cdot 10^{-5}$ \\
Higdon 1 & 0.0201 & 0.0202 & 0.0401 & 0.0099 & 0.0136 & 0.0189 \\
Higdon 2 & 0.0318 & 0.0318 & 0.0632 & 0.0164 & 0.0226 & 0.0316 \\
PML 1 & $3.1161 \cdot 10^{-6}$ & $6.3157 \cdot 10^{-5}$ & 0.0001 & $1.1581 \cdot 10^{-6}$ & $1.6438 \cdot 10^{-5}$ & $5.3302 \cdot 10^{-5}$ \\
PML 2 & $6.8821 \cdot 10^{-6}$ & $1.2520 \cdot 10^{-5}$ & $1.1478 \cdot 10^{-5}$ & $5.4974 \cdot 10^{-6}$ & $7.9195 \cdot 10^{-6}$ & $1.0318 \cdot 10^{-5}$ \\
S. Cúbico & 0.0578 & 0.2895 & 0.2972 & 0.0157 & 0.1376 & 0.2330 \\
S. Expoente & 0.1428 & 0.1899 & 0.2854 & 0.0519 & 0.1376 & 0.2150 \\
S. Linear & 0.1211 & 0.2525 & 0.2644 & 0.0382 & 0.1584 & 0.2248
\end{tabular}

Tabela 3.3: Erros para o segundo teste unidimensional. 
Como podemos ver, os menores erros são obtidos para as condições A1, HABC 1, HABC 2 e as PMLs, sendo que o erro decorrente da aplicação da PML para a equação de segunda ordem é menor do que os erros verificados para as demais condições de contorno.

\subsubsection{Desempenho das condições de contorno em relação ao refinamento da malha}

Nesta seção, analisamos os erros obtidos no decorrer do tempo para diferentes espaçamentos da malha para as condições abordadas na seção anterior.

\begin{tabular}{|c|c|c|c|c|c|c|c|}
\hline \multirow{2}{*}{ Método } & \multirow{2}{*}{$t$} & \multicolumn{3}{|c|}{ Erro absoluto na norma infinito } & \multicolumn{3}{|c|}{ Erro absoluto na norma 2} \\
\hline & & $\Delta x=0.01$ & $\begin{array}{l}\Delta x= \\
0.005\end{array}$ & $\begin{array}{c}\Delta x= \\
0.0025\end{array}$ & $\Delta x=0.01$ & $\begin{array}{l}\Delta x= \\
0.005\end{array}$ & $\begin{array}{c}\Delta x= \\
0.0025\end{array}$ \\
\hline \multirow{3}{*}{ Dirichlet } & 0.5 & 0.5 & 0.5 & 0.5 & 0.2351 & 0.2353 & 0.2353 \\
\hline & 1 & 1 & 1 & 1 & 0.4707 & 0.4707 & 0.4707 \\
\hline & 1.5 & 0.0033 & 0.0008 & 0.0002 & 0.0015 & 0.0004 & 0.0001 \\
\hline \multirow{3}{*}{ Cerjan } & 0.5 & 0.0030 & 0.1390 & 0.3631 & 0.0006 & 0.0362 & 0.1195 \\
\hline & 1 & 0.5 & 0.5030 & 0.7261 & 0.3104 & 0.3454 & 0.4113 \\
\hline & 1.5 & 0.7292 & 0.5 & 0.4755 & 0.4115 & 0.3312 & 0.2877 \\
\hline \multirow{3}{*}{ A1 } & 0.5 & $3.493 \cdot 10^{-4}$ & $2.197 \cdot 10^{-4}$ & $1.840 \cdot 10^{-4}$ & $2.049 \cdot 10^{-4}$ & $1.756 \cdot 10^{-4}$ & $1.672 \cdot 10^{-4}$ \\
\hline & 1 & $6.952 \cdot 10^{-4}$ & $4.395 \cdot 10^{-4}$ & $3.684 \cdot 10^{-4}$ & $4.073 \cdot 10^{-4}$ & $3.567 \cdot 10^{-4}$ & $3.406 \cdot 10^{-4}$ \\
\hline & 1.5 & $5.459 \cdot 10^{-4}$ & $4.023 \cdot 10^{-4}$ & $3.590 \cdot 10^{-4}$ & $3.995 \cdot 10^{-4}$ & $3.643 \cdot 10^{-4}$ & $3.494 \cdot 10^{-4}$ \\
\hline \multirow{3}{*}{ HABC 1} & 0.5 & $1.708 \cdot 10^{-4}$ & $1.413 \cdot 10^{-4}$ & $1.424 \cdot 10^{-4}$ & $6.333 \cdot 10^{-5}$ & $9.997 \cdot 10^{-5}$ & $1.261 \cdot 10^{-4}$ \\
\hline & 1 & $3.407 \cdot 10^{-4}$ & $2.825 \cdot 10^{-4}$ & $2.848 \cdot 10^{-4}$ & $1.664 \cdot 10^{-4}$ & $2.097 \cdot 10^{-4}$ & $2.584 \cdot 10^{-4}$ \\
\hline & 1.5 & $2.834 \cdot 10^{-4}$ & $2.520 \cdot 10^{-4}$ & $2.759 \cdot 10^{-4}$ & $1.764 \cdot 10^{-4}$ & $2.182 \cdot 10^{-4}$ & $2.669 \cdot 10^{-4}$ \\
\hline \multirow{3}{*}{ HABC 2} & 0.5 & $3.739 \cdot 10^{-5}$ & $6.887 \cdot 10^{-5}$ & $9.887 \cdot 10^{-5}$ & $1.872 \cdot 10^{-5}$ & $4.196 \cdot 10^{-5}$ & $8.446 \cdot 10^{-5}$ \\
\hline & 1 & $1.242 \cdot 10^{-4}$ & $1.377 \cdot 10^{-4}$ & $1.977 \cdot 10^{-4}$ & $6.390 \cdot 10^{-5}$ & $9.767 \cdot 10^{-5}$ & $1.748 \cdot 10^{-4}$ \\
\hline & 1.5 & $1.570 \cdot 10^{-4}$ & $1.364 \cdot 10^{-4}$ & $1.910 \cdot 10^{-4}$ & $8.554 \cdot 10^{-5}$ & $1.069 \cdot 10^{-4}$ & $1.825 \cdot 10^{-4}$ \\
\hline \multirow{3}{*}{ Higdon 1} & 0.5 & 0.0201 & 0.02 & 0.02 & 0.0099 & 0.0096 & 0.0095 \\
\hline & 1 & 0.0401 & 0.04 & 0.04 & 0.0189 & 0.0189 & 0.0188 \\
\hline & 1.5 & 0.0210 & 0.0209 & 0.0209 & 0.0096 & 0.0097 & 0.0098 \\
\hline \multirow{3}{*}{ Higdon 2} & 0.5 & 0.0318 & 0.0313 & 0.0311 & 0.0164 & 0.0155 & 0.0151 \\
\hline & 1 & 0.0632 & 0.0626 & 0.0623 & 0.0316 & 0.0309 & 0.0305 \\
\hline & 1.5 & 0.0401 & 0.0382 & 0.0374 & 0.0224 & 0.0212 & 0.0208 \\
\hline \multirow{3}{*}{ PML 1} & 0.5 & $6.065 \cdot 10^{-3}$ & $3.052 \cdot 10^{-3}$ & $1.626 \cdot 10^{-3}$ & $3.331 \cdot 10^{-3}$ & $1.682 \cdot 10^{-3}$ & $9.130 \cdot 10^{-4}$ \\
\hline & 1 & $5.184 \cdot 10^{-4}$ & $5.217 \cdot 10^{-4}$ & $7.589 \cdot 10^{-4}$ & $3.249 \cdot 10^{-4}$ & $3.595 \cdot 10^{-4}$ & $4.279 \cdot 10^{-4}$ \\
\hline & 1.5 & $7.308 \cdot 10^{-4}$ & $5.181 \cdot 10^{-4}$ & $5.184 \cdot 10^{-4}$ & $4.216 \cdot 10^{-4}$ & $3.434 \cdot 10^{-4}$ & $3.216 \cdot 10^{-4}$ \\
\hline \multirow{3}{*}{ PML 2} & 0.5 & $6.882 \cdot 10^{-6}$ & $2.056 \cdot 10^{-5}$ & $4.035 \cdot 10^{-5}$ & $5.497 \cdot 10^{-6}$ & $1.813 \cdot 10^{-5}$ & $3.682 \cdot 10^{-5}$ \\
\hline & 1 & $1.148 \cdot 10^{-5}$ & $3.992 \cdot 10^{-5}$ & $8.013 \cdot 10^{-5}$ & $1.031 \cdot 10^{-5}$ & $3.688 \cdot 10^{-5}$ & $7.515 \cdot 10^{-5}$ \\
\hline & 1.5 & $1.148 \cdot 10^{-5}$ & $3.998 \cdot 10^{-5}$ & $8.017 \cdot 10^{-5}$ & $1.096 \cdot 10^{-5}$ & $3.970 \cdot 10^{-5}$ & $8.001 \cdot 10^{-5}$ \\
\hline \multirow{3}{*}{ S. Cúbico } & 0.5 & 0.0578 & 0.1444 & 0.3630 & 0.0157 & 0.0386 & 0.1195 \\
\hline & 1 & 0.2972 & 0.4914 & 0.7259 & 0.2330 & 0.3425 & 0.4113 \\
\hline & 1.5 & 0.3798 & 0.4872 & 0.4752 & 0.3096 & 0.3265 & 0.2876 \\
\hline \multirow{3}{*}{ S. Expoente } & 0.5 & 0.1428 & 0.1982 & 0.3562 & 0.0519 & 0.0671 & 0.1233 \\
\hline & 1 & 0.2854 & 0.4067 & 0.7123 & 0.2150 & 0.3181 & 0.4039 \\
\hline & 1.5 & 0.3261 & 0.3687 & 0.4284 & 0.2608 & 0.2771 & 0.2646 \\
\hline \multirow{3}{*}{ S. Linear } & 0.5 & 0.1211 & 0.1736 & 0.3612 & 0.0382 & 0.0523 & 0.1202 \\
\hline & 1 & 0.2644 & 0.4366 & 0.7224 & 0.2248 & 0.3284 & 0.4097 \\
\hline & 1.5 & 0.3105 & 0.4233 & 0.4653 & 0.2717 & 0.3015 & 0.2828 \\
\hline
\end{tabular}

Tabela 3.4: Erro para as condições de contorno com diversos espaçamentos de malha.

Como vemos nas tabelas anteriores, as condições de absorção não foram consideravelmente 
afetadas pelo refinamento da malha, resultando em erros próximos para todos os espaçamentos adotados. Já algumas camadas de amortecimento, como as de Sochacki e a PML 2, tiveram sua eficácia alterada conforme o espaçamento foi reduzido (e, consequentemente, também foi diminuída a espessura total das camadas de amortecimento). Isso indica que a espessura de tais camadas pode alterar o desempenho do método e requer atenção na aplicação das condições.

\subsection{Equação da onda bidimensional - Exemplo 1}

O teste realizado nesta seção é uma adaptação do primeiro exemplo apresentado em [GSZY17], trabalho que se destina à comparação entre condições de contorno para a equação da onda acústica. Neste exemplo, é apresentada a resolução numérica da seguinte equação:

$$
\begin{aligned}
u_{t t}-2500\left(u_{x x}+u_{y y}\right) & =f(t) \delta\left(\mathbf{x}-\mathbf{x}_{0}\right),(x, y) \in[0,1500] \times[0,1500] \\
u(x, y, 0) & =0 \\
u_{t}(x, y, 0) & =0
\end{aligned}
$$

onde

$$
f(t)=\left(1-800 \pi^{2} t^{2}\right) e^{-400 \pi^{2} t^{2}}
$$

e

$$
\delta(\mathbf{x})=\left\{\begin{array}{l}
1, \text { se } \mathbf{x}=0, \\
0, \text { caso contrário }
\end{array}\right.
$$

$\mathrm{e} \mathbf{x}_{0}=(750,750)$

O gráfico de $f(t)$ é o seguinte:

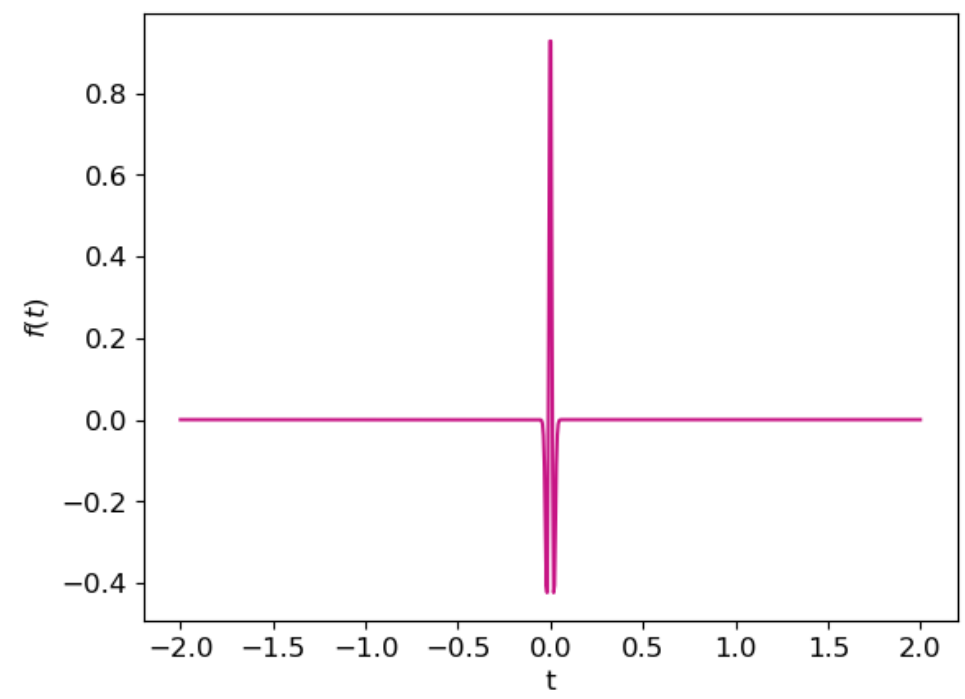

Figura 3.29: Gráfico de $f(t)$

Para este exemplo, utilizamos uma fonte pontual atuando no meio do domínio, com condições iniciais nulas. Nesse caso, os efeitos do truncamento do domínio serão perceptíveis apenas após $t=0.3$. Os valores adotados no teste são

- Tempo total de simulação: $T=0.5$;

- Malha de $251 \times 251$ pontos no espaço;

- $\Delta x=6, \Delta y=6, \Delta t=0.001, \lambda=c \frac{\Delta t}{\Delta x} \approx 0.4167$. 
Na primeira sequência de figuras a seguir, temos a representação gráfica da solução de referência no domínio de interesse $[0,1500] \times[0,1500]$. Nas imagens seguintes, apresentamos a solução no domínio estendido $[-750,2250] \times[-750,2250]$ :

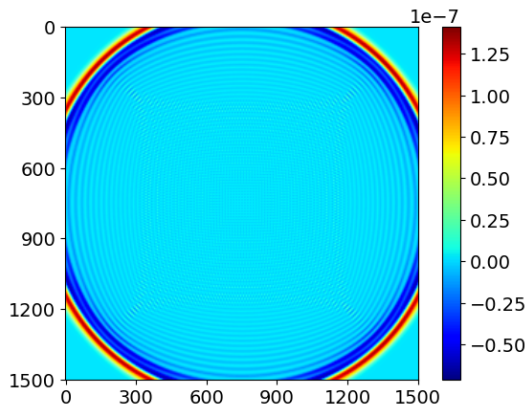

(a) $t=0.35$

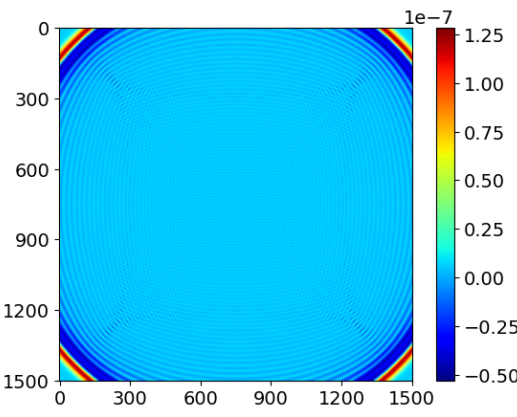

(b) $t=0.4$

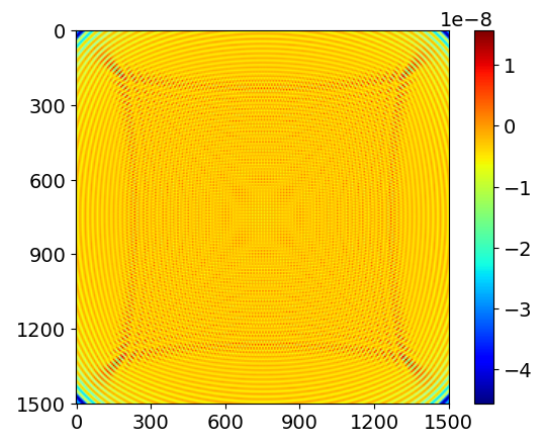

(c) $t=0.45$

Figura 3.30: Solução tomada como referência para o primeiro exemplo bidimensional no domínio de interesse.

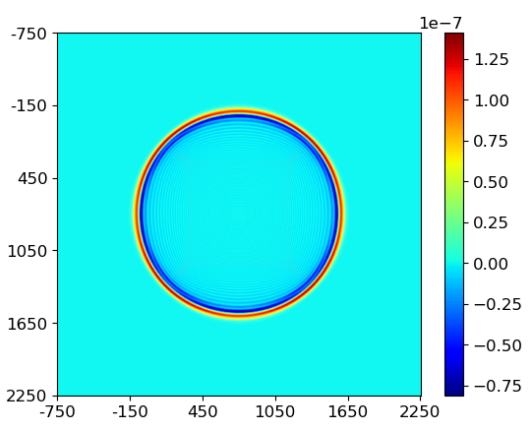

(a) $t=0.35$

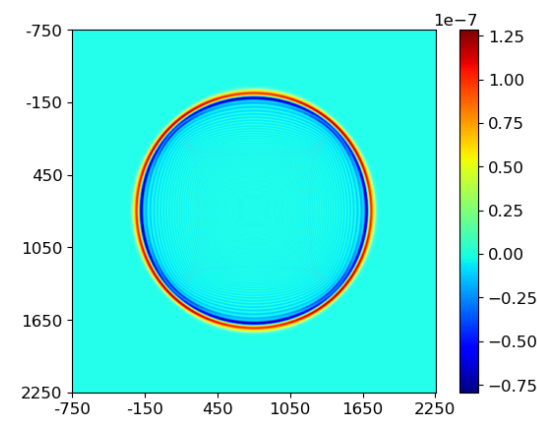

(b) $t=0.4$

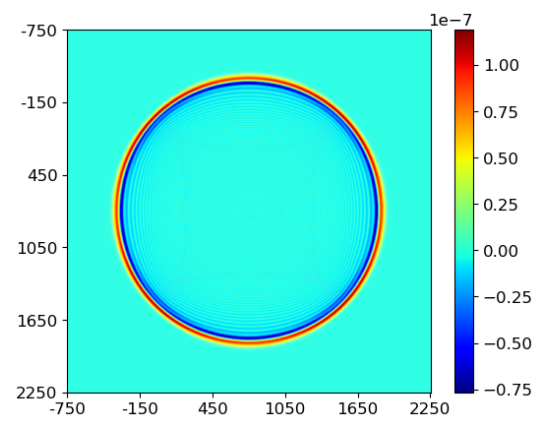

(c) $t=0.45$

Figura 3.31: Solução tomada como referência para o primeiro exemplo bidimensional no domínio estendido.

Na primeira seção, apresentamos os resultados obtidos para cada condição de contorno abordada anteriormente adotando, para as camadas de amortecimento, camadas de espessura igual a 20 pontos da malha, e, na segunda seção, utilizamos outros valores para a espessura das camadas. Novamente, tomamos as seguintes medidas de erro:

$$
\frac{\left\|e^{n}\right\|_{\infty}}{\left\|u^{n}\right\|_{\infty}}=\frac{\sup _{i, j}\left\{\left|u_{i, j}^{*^{n}}-u_{i, j}^{n}\right|\right\}}{\sup _{i, j}\left\{\left|u_{i, j}^{*^{n}}\right|\right\}},\left\|e^{n}\right\|_{2}=\left(\frac{\sum_{i=0}^{N} \sum_{j=0}^{N}\left|u_{i, j}^{*^{n}}-u_{i, j}^{n}\right|^{2}}{M}\right)^{\frac{1}{2}},
$$

com $M$ sendo o número de pontos da malha.

Os parâmetros adotados para a realização deste teste são:

- Para a primeira seção, $L=20$ e a espessura da camada de amortecimento é $\delta=120$. Para a segunda seção, incluímos os casos $L=10, \operatorname{com} \delta=60$ e $L=40$, com $\delta=240$;

- Cerjan: $\tilde{\lambda}=0.015$;

- HABC 2: $M=2, \alpha=1.5+0.07(L-M)$;

- Condição de Higdon de primeira ordem: $\alpha=\frac{\pi}{4}, a=b=0.5$;

- Condição de Higdon de segunda ordem: $\alpha_{1}=0, \alpha_{2}=\frac{\pi}{4}, a_{1}=a_{2}=b_{1}=b_{2}=0.5$; 
- PML para o sistema de primeira ordem: $R=10^{-4}$,

$$
d(x)=\left\{\begin{array}{l}
\frac{3}{40 \Delta x} \ln \left(\frac{1}{0.0001}\right)\left(\frac{x}{20 \Delta x}\right)^{2}, \text { se } x<0 \\
\frac{3}{40 \Delta x} \ln \left(\frac{1}{0.0001}\right)\left(\frac{x-1500}{20 \Delta x}\right)^{2}, \text { se } x>1500
\end{array}\right.
$$

- PML para a equação de segunda ordem: $R=10^{-7}$,

$$
d(x)=\left\{\begin{array}{l}
\frac{3}{40 \Delta x} \ln \left(\frac{1}{10^{-7}}\right)\left(\frac{x}{20 \Delta x}\right)^{2}, \text { se } x<0 \\
\frac{3}{40 \Delta x} \ln \left(\frac{1}{10^{-7}}\right)\left(\frac{x-1500}{20 \Delta x}\right)^{2}, \text { se } x>1500
\end{array}\right.
$$

- Condição de Sochacki com amortecedor cúbico: $S=1$;

- Condição de Sochacki com amortecedor expoente: $S=0.5$;

- Condição de Sochacki com amortecedor linear: $S=10$.

\subsubsection{Resultados gerais}

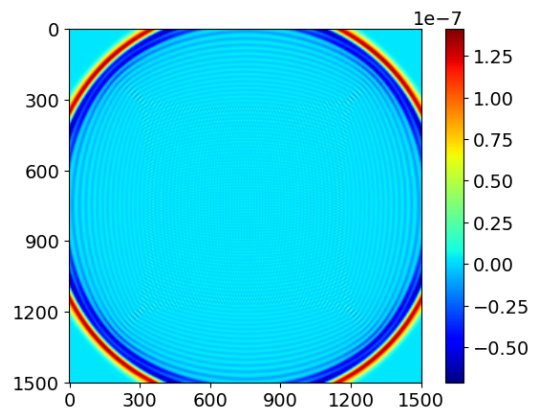

(a) $t=0.35$

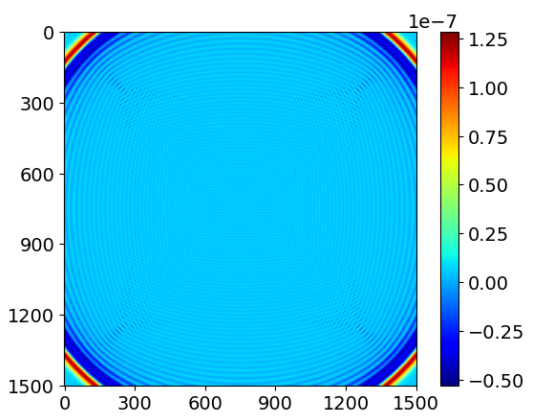

(b) $t=0.4$

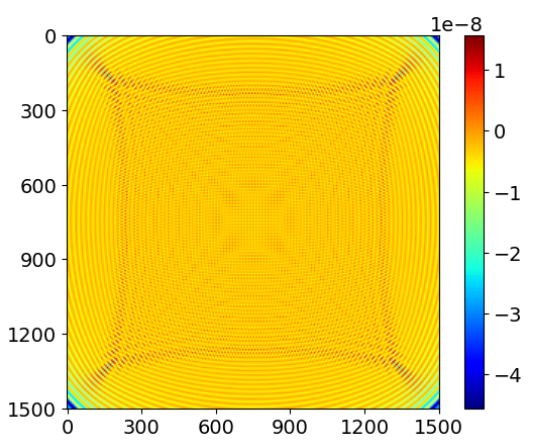

(c) $t=0.45$

Figura 3.32: Solução tomada como referência para o primeiro exemplo bidimensional.

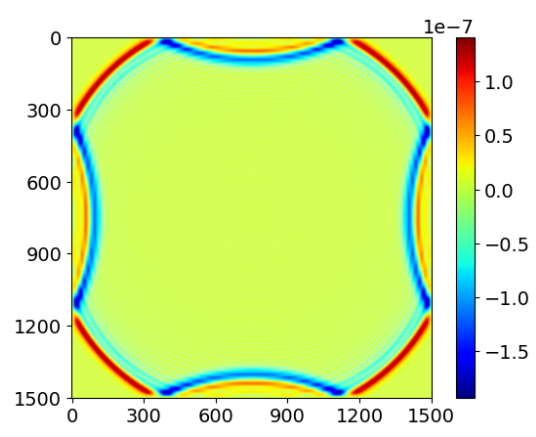

(a) $t=0.35$

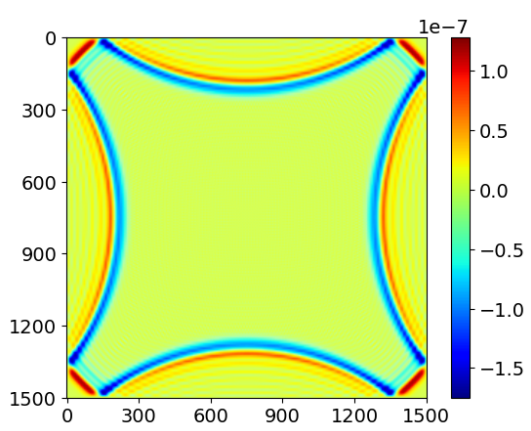

(b) $t=0.4$

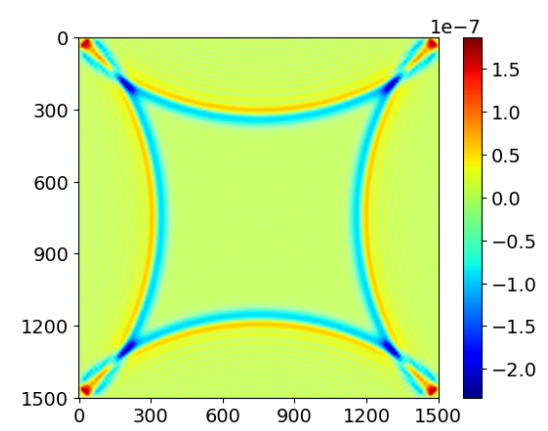

(c) $t=0.45$

Figura 3.33: Condição de Dirichlet $u=0$ aplicada ao primeiro exemplo bidimensional. 


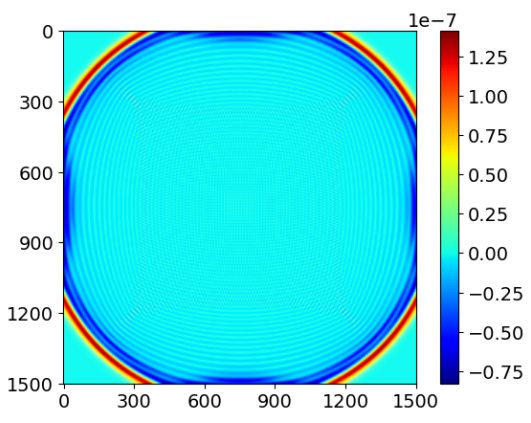

(a) $t=0.35$

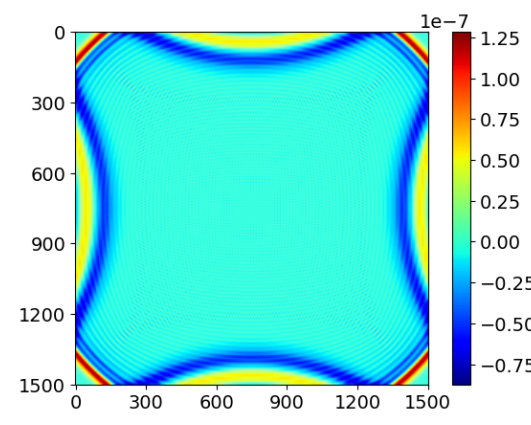

(b) $t=0.4$

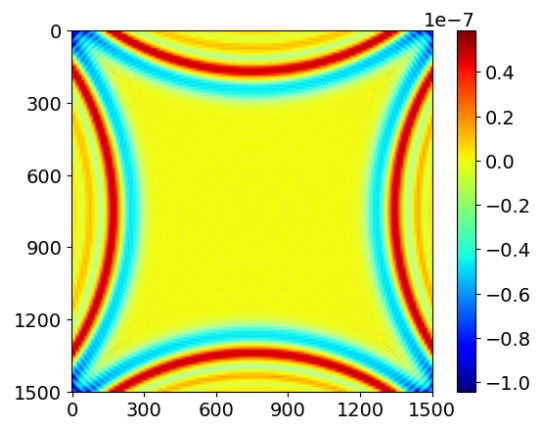

(c) $t=0.45$

Figura 3.34: Condição de Cerjan, com $\tilde{\lambda}=0.015$, aplicada ao primeiro exemplo bidimensional.

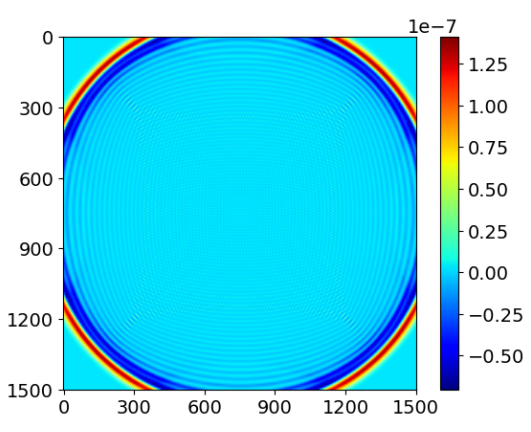

(a) $t=0.35$

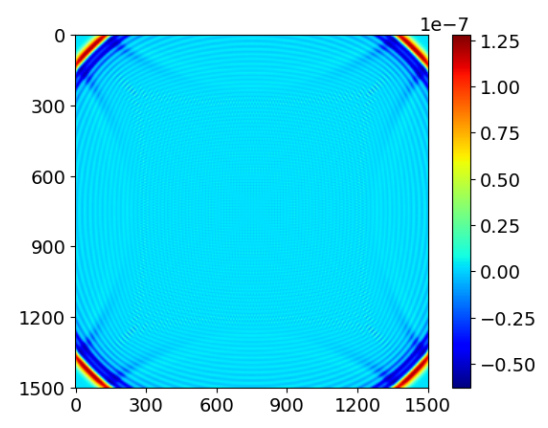

(b) $t=0.4$

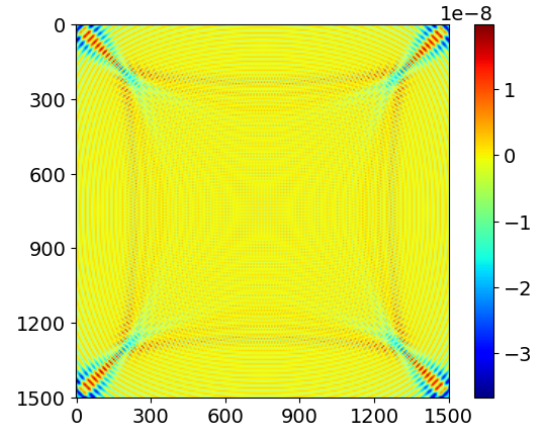

(c) $t=0.45$

Figura 3.35: Condição A1 aplicada ao primeiro exemplo bidimensional.

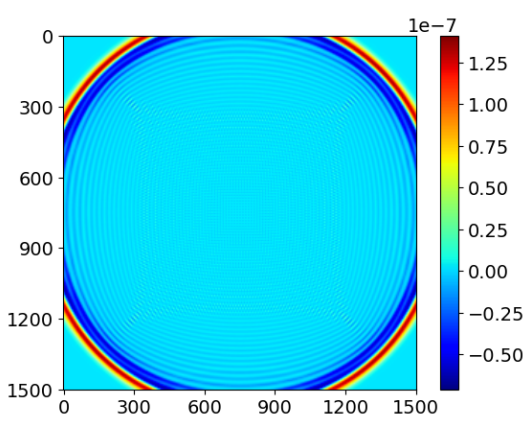

(a) $t=0.35$

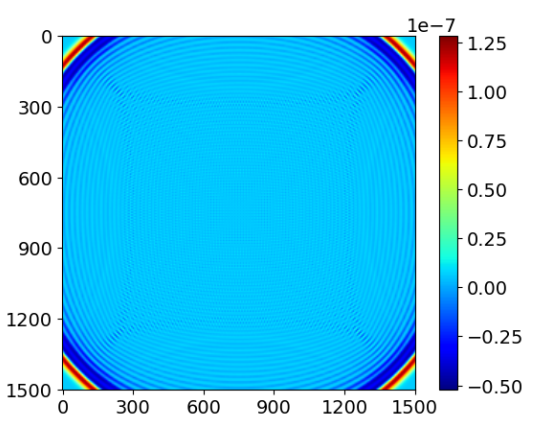

(b) $t=0.4$

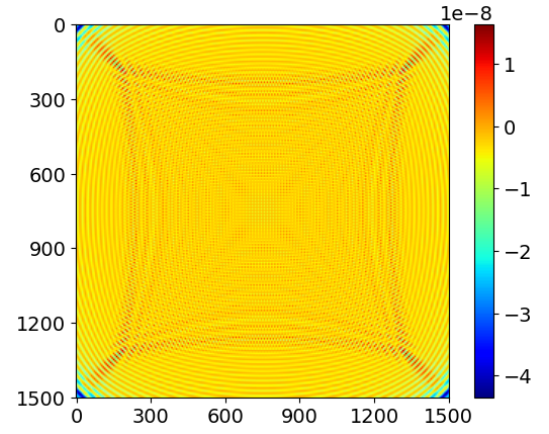

(c) $t=0.45$

Figura 3.36: Condição A2 aplicada ao primeiro exemplo bidimensional. 


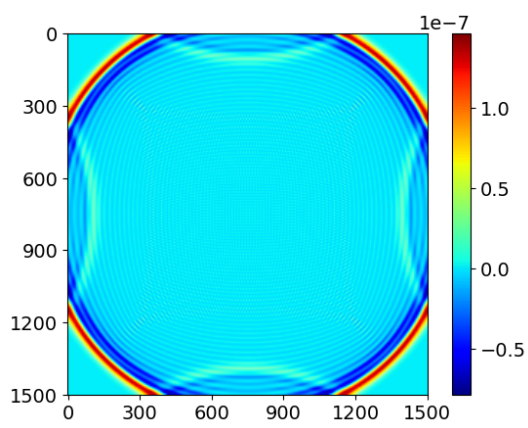

(a) $t=0.35$

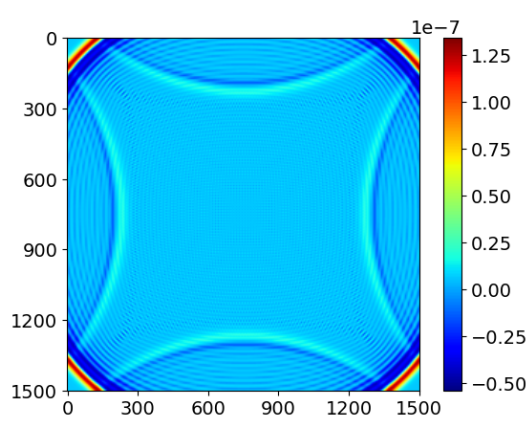

(b) $t=0.4$

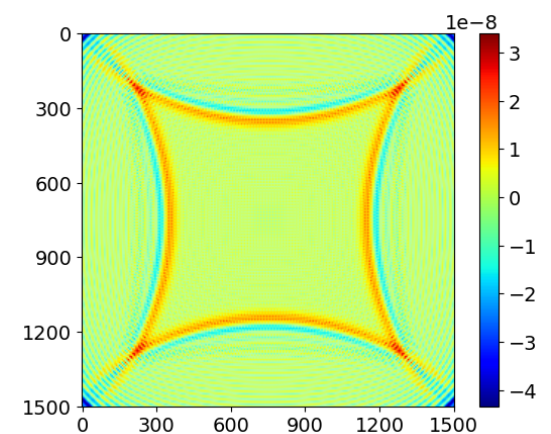

(c) $t=0.45$

Figura 3.37: Condição de Higdon de primeira ordem, com $\alpha=\frac{\pi}{4}, a=b=0.5$, aplicada ao primeiro exemplo bidimensional.

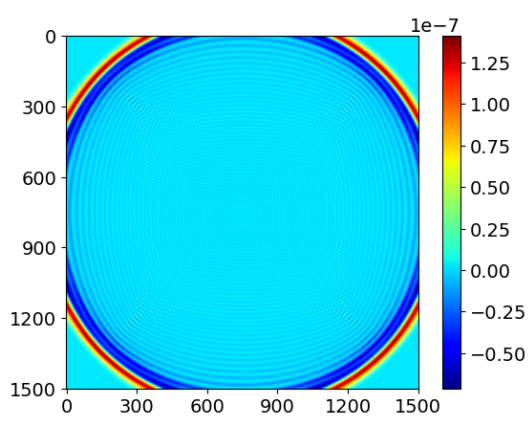

(a) $t=0.35$

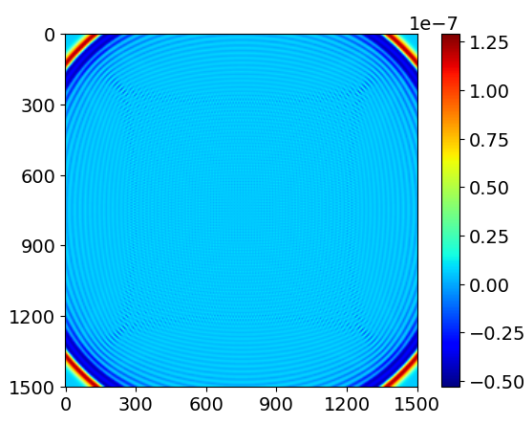

(b) $t=0.4$

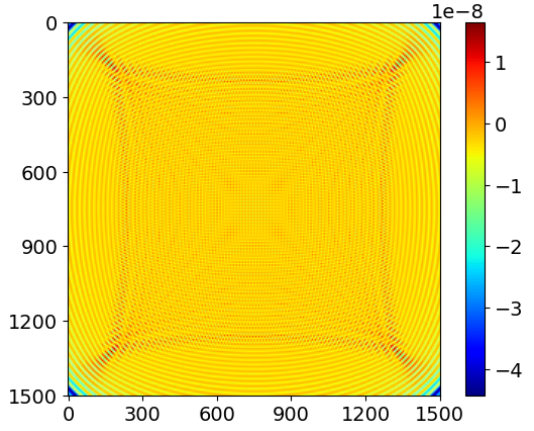

(c) $t=0.45$

Figura 3.38: Condição de Higdon de segunda ordem, com $\alpha_{1}=0, \alpha_{2}=\frac{\pi}{4}, a_{1}=a_{2}=b_{1}=b_{2}=0.5$, aplicada ao primeiro exemplo bidimensional.

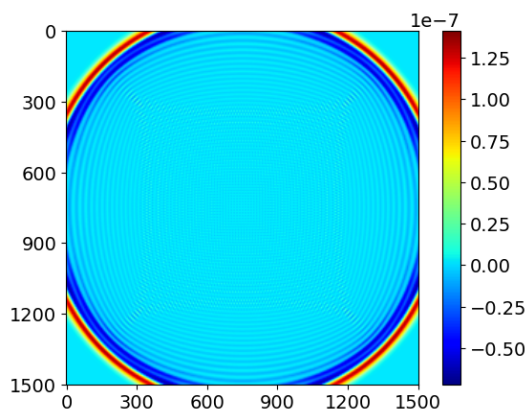

(a) $t=0.35$

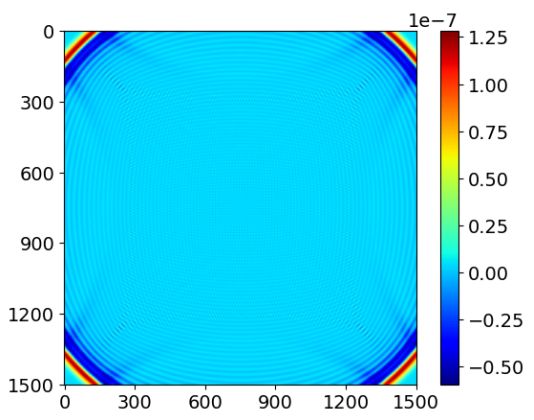

(b) $t=0.4$

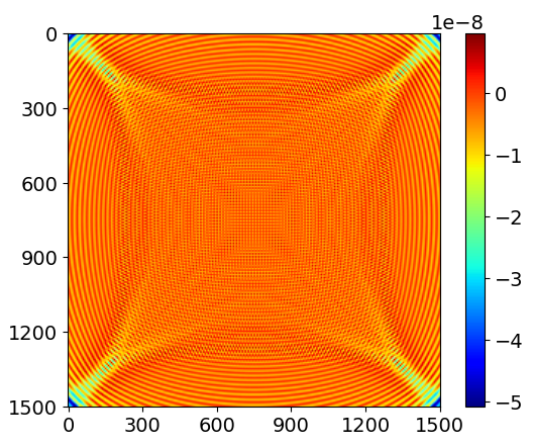

(c) $t=0.45$

Figura 3.39: HABC original com a condição A1 aplicada ao primeiro exemplo bidimensional. 


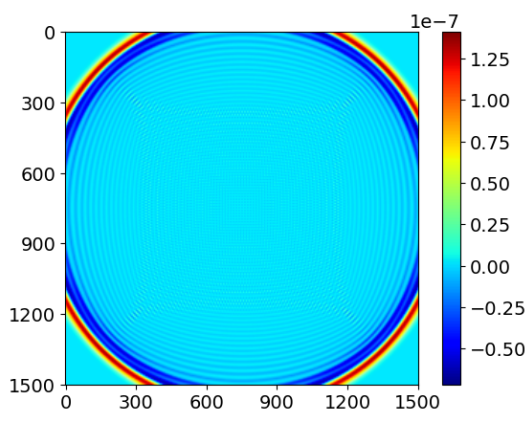

(a) $t=0.35$

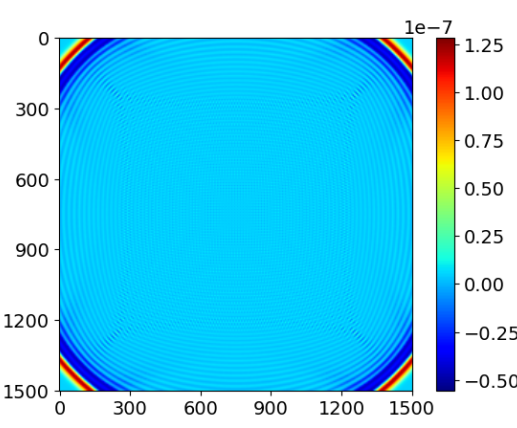

(b) $t=0.4$

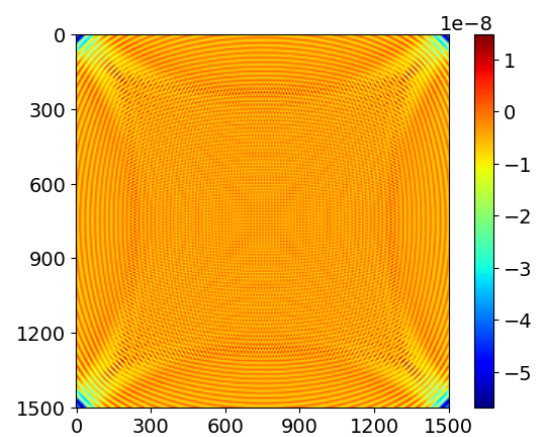

(c) $t=0.45$

Figura 3.40: $H A B C$ modificada com a condição A1, com $M=2, \alpha=1.5+0.07(L-M)$, aplicada ao primeiro exemplo bidimensional.

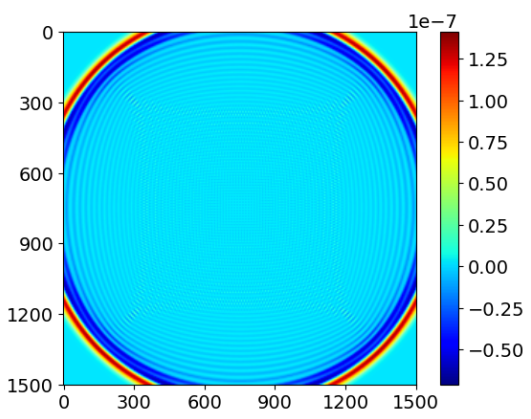

(a) $t=0.35$

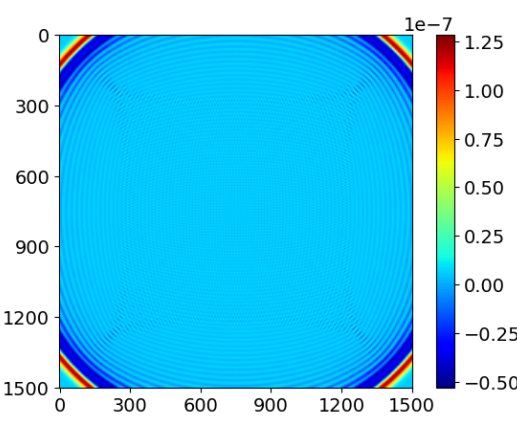

(b) $t=0.4$

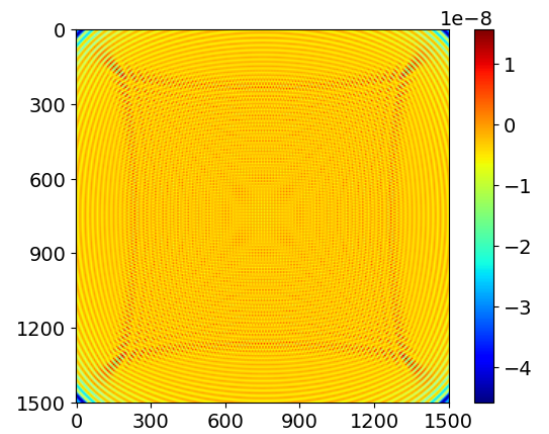

(c) $t=0.45$

Figura 3.41: HABC original com a condição A2 aplicada ao primeiro exemplo bidimensional.

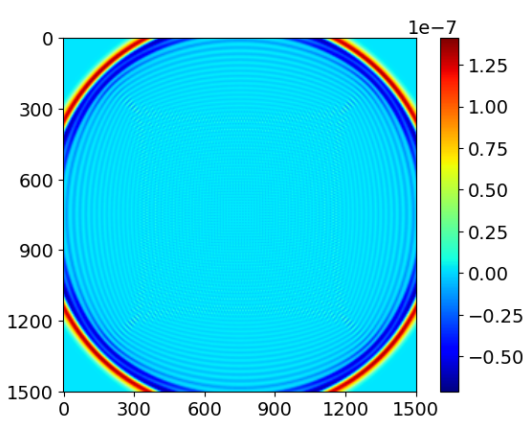

(a) $t=0.35$

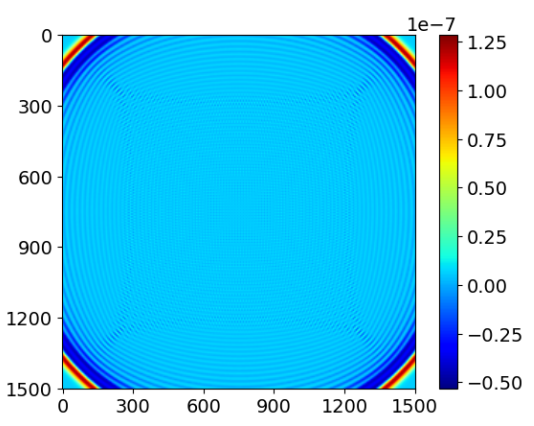

(b) $t=0.4$

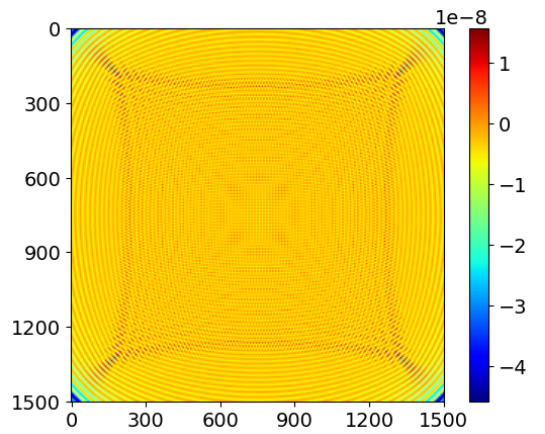

(c) $t=0.45$

Figura 3.42: $H A B C$ modificada com a condição A2, com $M=2$ e $\alpha=1.5+0.07(L-M)$, aplicada ao primeiro exemplo bidimensional. 


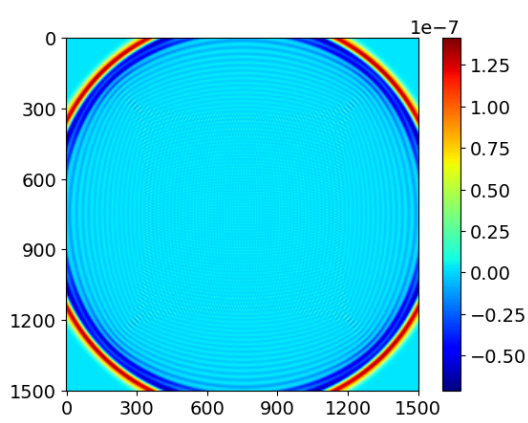

(a) $t=0.35$

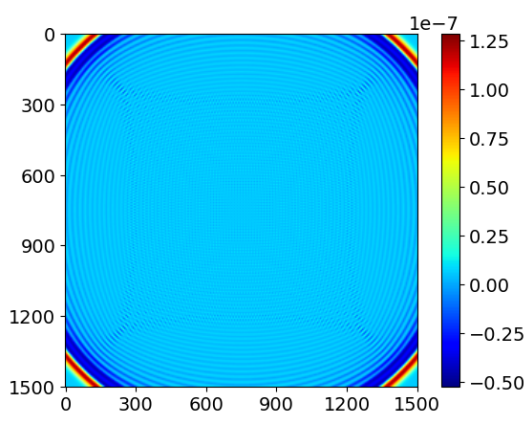

(b) $t=0.4$

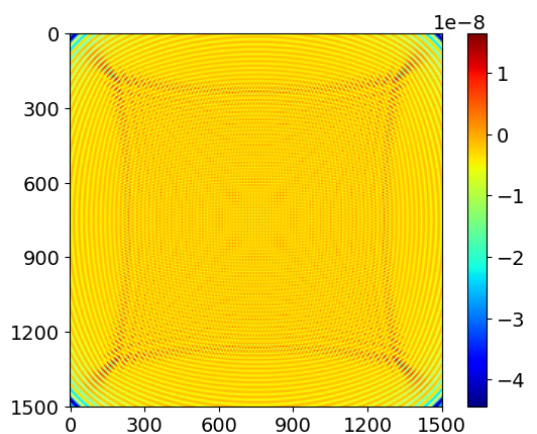

(c) $t=0.45$

Figura 3.43: $H A B C$ original com a condição de Higdon de segunda ordem, $\alpha_{1}=0, \alpha_{2}=\frac{\pi}{4}$ e $a_{1}=a_{2}=$ $b_{1}=b_{2}=0.5$, aplicada ao primeiro exemplo bidimensional.

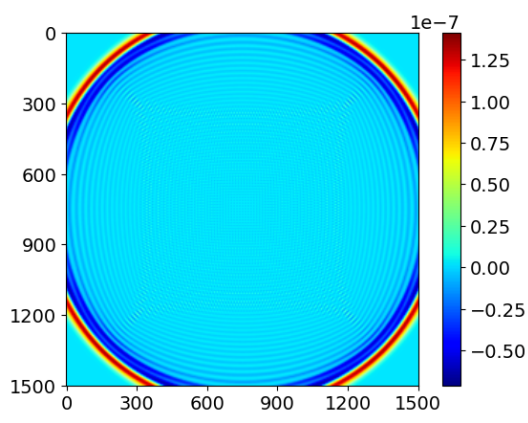

(a) $t=0.35$

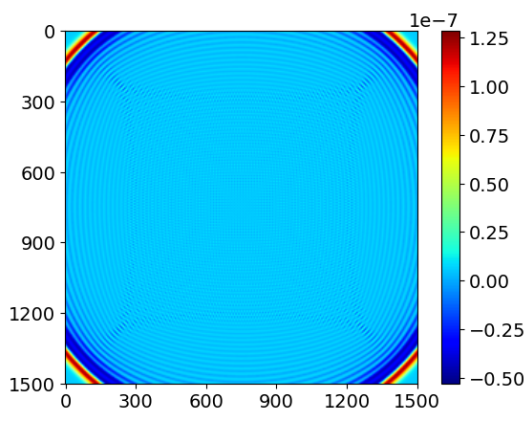

(b) $t=0.4$

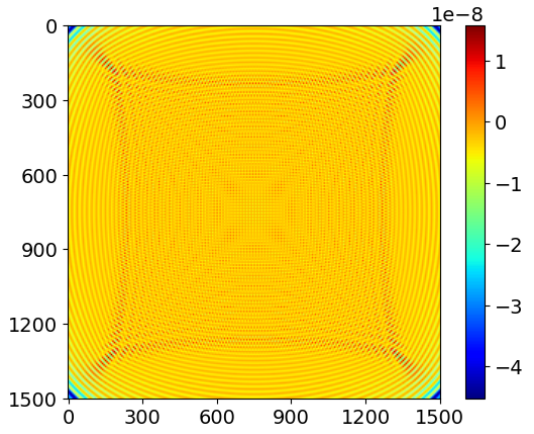

(c) $t=0.45$

Figura 3.44: HABC modificada com a condição de Higdon de segunda ordem, com $M=2, \alpha=1.5+$ $0.07(L-M), \alpha_{1}=0, \alpha_{2}=\frac{\pi}{4}$ e $a_{1}=a_{2}=b_{1}=b_{2}=0.5$, aplicada ao primeiro exemplo bidimensional.

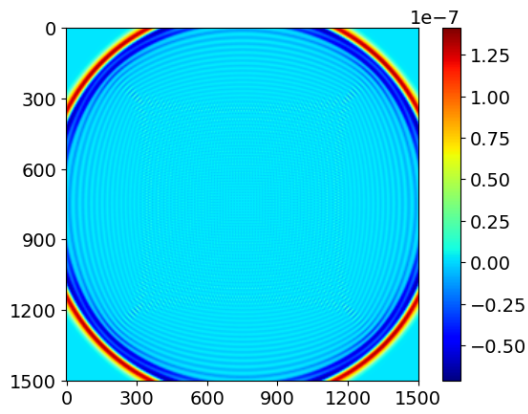

(a) $t=0.35$

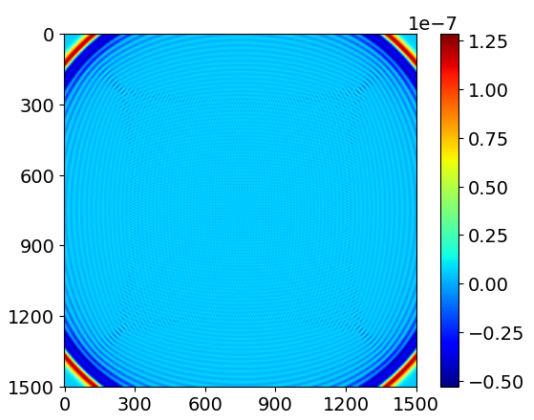

(b) $t=0.4$

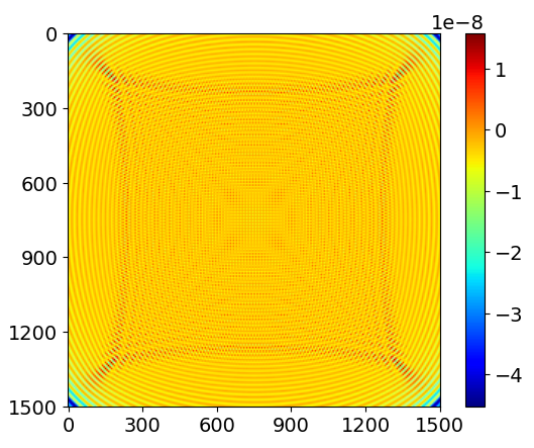

(c) $t=0.45$

Figura 3.45: Condição PML para o sistema de primeira ordem, com $R=10^{-4}$, aplicada ao primeiro exemplo bidimensional. 


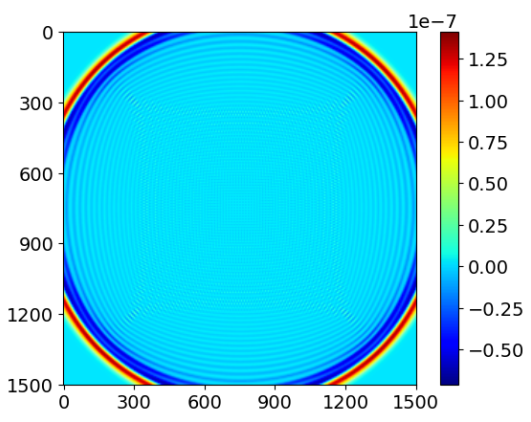

(a) $t=0.35$

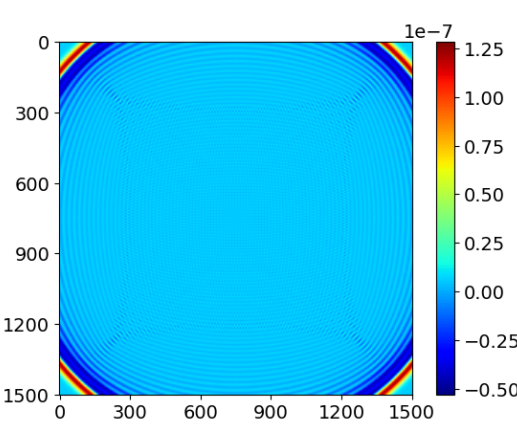

(b) $t=0.4$

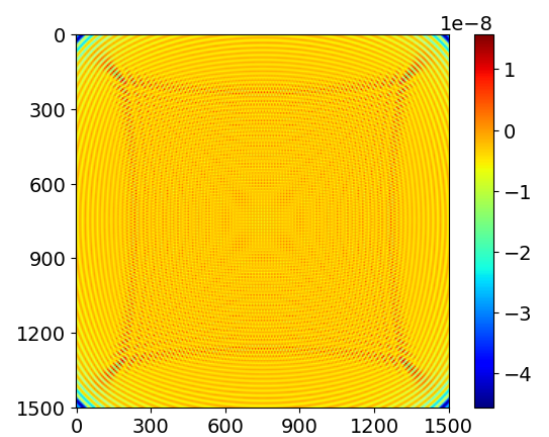

(c) $t=0.45$

Figura 3.46: Condição PML para a equação de segunda ordem, com $R=10^{-7}$, aplicada ao primeiro exemplo bidimensional.

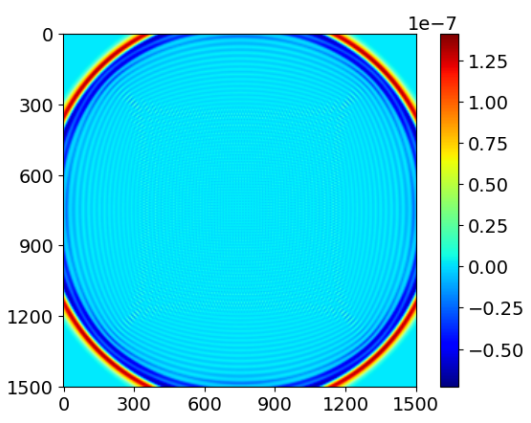

(a) $t=0.35$

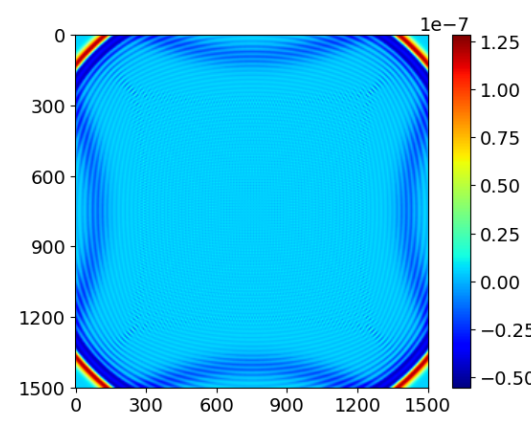

(b) $t=0.4$

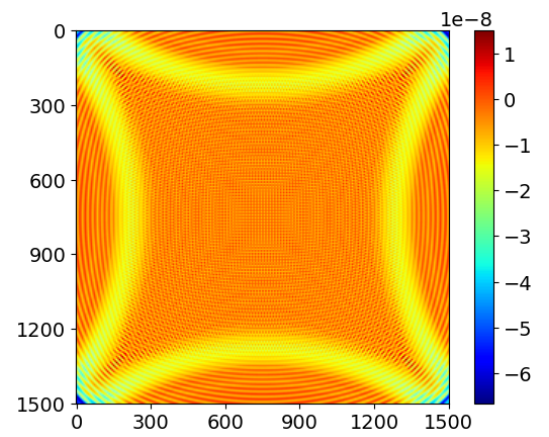

(c) $t=0.45$

Figura 3.47: Condição de Sochacki com amortecedor cúbico, com $S=1$, aplicada ao primeiro exemplo bidimensional.

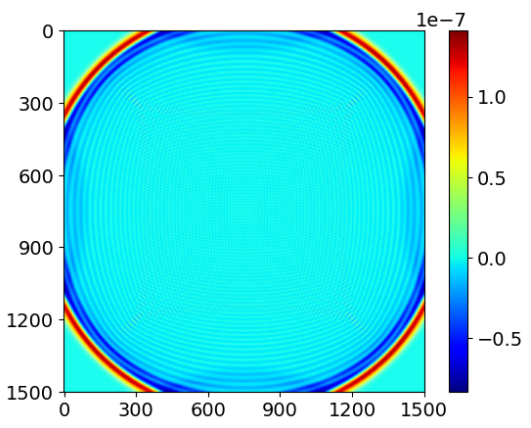

(a) $t=0.35$

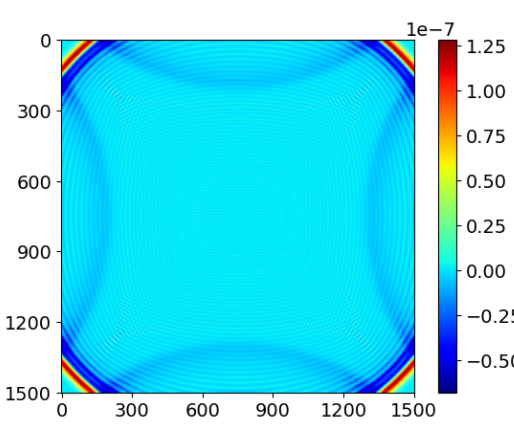

(b) $t=0.4$

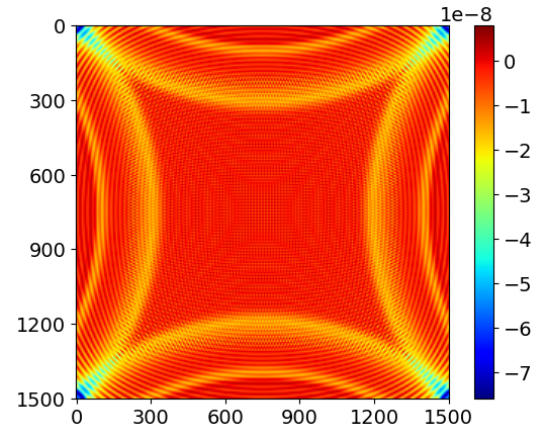

(c) $t=0.45$

Figura 3.48: Condição de Sochacki com amortecedor expoente, com $S=0.5$, aplicada ao primeiro exemplo bidimensional. 


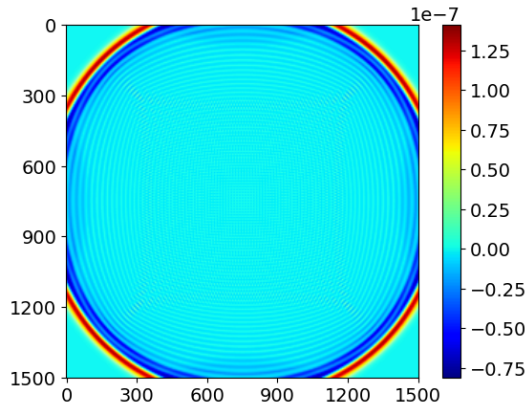

(a) $t=0.35$

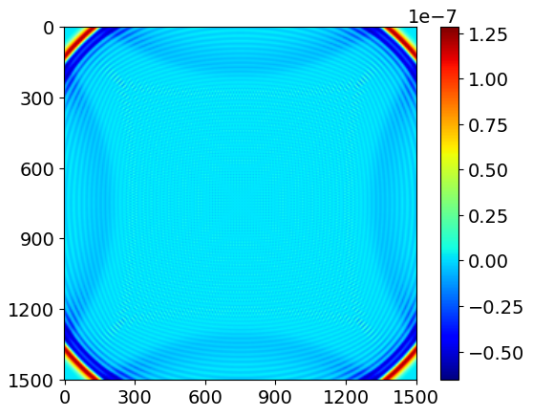

(b) $t=0.4$

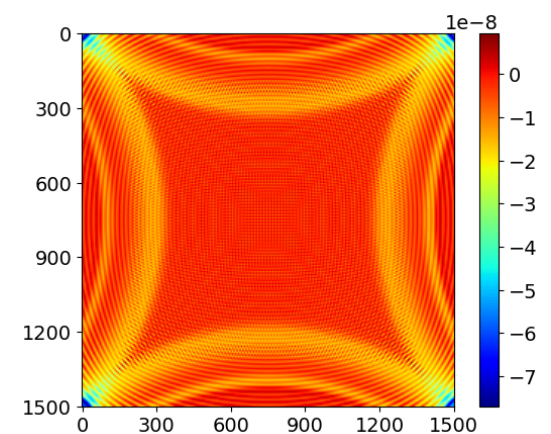

(c) $t=0.45$

Figura 3.49: Condição de Sochacki com amortecedor linear, com $S=10$, aplicada ao primeiro exemplo bidimensional.

\begin{tabular}{c|ccc|ccc}
\multirow{2}{*}{ Método } & \multicolumn{3}{|c|}{ Erro relativo na norma infinito } & \multicolumn{3}{c}{ Erro absoluto na norma 2 } \\
& $t=0.35$ & $t=0.4$ & $t=0.45$ & $t=0.35$ & $t=0.4$ & $t=0.45$ \\
\hline Dirichlet & 0.9202 & 0.9890 & 4.8575 & $2.647 \cdot 10^{-8}$ & $3.226 \cdot 10^{-8}$ & $3.377 \cdot 10^{-8}$ \\
Cerjan & 0.3771 & 0.4532 & 1.3945 & $6.289 \cdot 10^{-9}$ & $1.874 \cdot 10^{-8}$ & $2.406 \cdot 10^{-8}$ \\
A1 & 0.0493 & 0.1202 & 0.4114 & $7.124 \cdot 10^{-10}$ & $1.785 \cdot 10^{-9}$ & $2.074 \cdot 10^{-9}$ \\
A2 & 0.0109 & 0.0144 & 0.1406 & $2.634 \cdot 10^{-10}$ & $3.884 \cdot 10^{-10}$ & $5.838 \cdot 10^{-10}$ \\
Higdon 1 & 0.0108 & 0.1493 & 0.5830 & $4.400 \cdot 10^{-9}$ & $4.686 \cdot 10^{-9}$ & $4.782 \cdot 10^{-9}$ \\
Higdon 2 & 0.0076 & 0.0103 & 0.0376 & $1.240 \cdot 10^{-10}$ & $2.143 \cdot 10^{-10}$ & $2.609 \cdot 10^{-10}$ \\
HABC 1 com A1 & 0.0217 & 0.0673 & 0.2732 & $3.354 \cdot 10^{-10}$ & $1.061 \cdot 10^{-9}$ & $1.493 \cdot 10^{-9}$ \\
HABC 2 com A1 & 0.0062 & 0.0285 & 0.2645 & $9.919 \cdot 10^{-11}$ & $5.266 \cdot 10^{-10}$ & $1.242 \cdot 10^{-9}$ \\
HABC 1 com A2 & $3.979 \cdot 10^{-4}$ & 0.0013 & 0.0147 & $1.005 \cdot 10^{-11}$ & $2.279 \cdot 10^{-11}$ & $4.235 \cdot 10^{-11}$ \\
HABC 2 com A2 & $3.393 \cdot 10^{-5}$ & $3.164 \cdot 10^{-4}$ & 0.0067 & $6.191 \cdot 10^{-12}$ & $7.018 \cdot 10^{-12}$ & $2.923 \cdot 10^{-11}$ \\
HABC 1 com & 0.0021 & 0.0043 & 0.0269 & $3.995 \cdot 10^{-11}$ & $1.008 \cdot 10^{-10}$ & $1.762 \cdot 10^{-10}$ \\
Higdon 2 & & & & & & \\
HABC 2 com & $5.488 \cdot 10^{-4}$ & 0.0021 & 0.0097 & $7.656 \cdot 10^{-12}$ & $5.384 \cdot 10^{-11}$ & $1.039 \cdot 10^{-10}$ \\
Higdon 2 & & & & & & \\
PML 1 & $1.602 \cdot 10^{-4}$ & $7.097 \cdot 10^{-4}$ & 0.0071 & $2.670 \cdot 10^{-12}$ & $1.400 \cdot 10^{-11}$ & $3.618 \cdot 10^{-11}$ \\
PML 2 & $1.449 \cdot 10^{-4}$ & $4.334 \cdot 10^{-4}$ & 0.0016 & $2.334 \cdot 10^{-12}$ & $8.472 \cdot 10^{-12}$ & $1.187 \cdot 10^{-11}$ \\
S. Cúbico & 0.0771 & 0.1287 & 0.6402 & $1.210 \cdot 10^{-9}$ & $4.924 \cdot 10^{-9}$ & $6.296 \cdot 10^{-9}$ \\
S. Expoente & 0.0891 & 0.1162 & 0.6778 & $1.343 \cdot 10^{-9}$ & $4.112 \cdot 10^{-9}$ & $5.776 \cdot 10^{-9}$ \\
S. Linear & 0.0743 & 0.0996 & 0.7192 & $2.336 \cdot 10^{-9}$ & $3.906 \cdot 10^{-9}$ & $5.377 \cdot 10^{-9}$
\end{tabular}

Tabela 3.5: Erros para o primeiro teste bidimensional.

Nas figuras 3.32a, 3.32b e 3.32c, temos a representação gráfica da solução tomada como referência para a verificação da eficácia dos métodos, calculada em um domínio suficientemente estendido. As figuras seguintes apresentam os resultados obtidos por meio da aplicação da condição de Dirichlet e mostram que, assim como no caso unidimensional, as reflexões causadas pelo truncamento do domínio são incompatíveis com a simulação do fenômeno desejado, isto é, a propagação da onda em um domínio ilimitado.

A condição de Cerjan fornece resultados melhores, com certa atenuação das ondas refletidas. Para $t=0.35$, as reflexões são visíveis, embora atenuadas, e o contraste entre a solução de referência e a resultante da aplicação do método é menor para a condição de Cerjan do que para a condição de Dirichlet. O mesmo ocorre com as soluções para $t=0.4$ e $t=0.45$, embora ainda não seja possível considerar o resultado obtido semelhante ao esperado.

A condição A1 resulta em reflexões menores do que as obtidas para os métodos anteriores em todos os valores de $t$ observados, embora seus efeitos ainda sejam perceptíveis; por sua vez, a aplicação da condição A2, cuja implementação é mais complexa por contar com derivadas temporais 
de ordem 2, resulta em um comportamento mais próximo do esperado. Os resultados apresentados nas figuras 3.39a, 3.39b e 3.39c e 3.40a, 3.40b e 3.40c se referem à aplicação das HABCs original e modificada nas quais utilizamos a condição A1 para aproximação da OWWE. Os resultados para a versão modificada são melhores do que os obtidos para a original que, por sua vez, apresenta desempenho superior à condição A1.

A condição A2, bem como as HABCs associadas, apresenta resultados superiores às condições avaliadas anteriormente, e as diferenças entre esses métodos não podem ser verificadas com facilidade por meio da representação gráfica das soluções. O mesmo ocorre com a condição de Higdon de segunda ordem e as HABCs associadas. Já a versão de primeira ordem da condição de Higdon apresentou resultados inferiores às demais condições mencionadas, superando apenas as condições de Cerjan e Dirichlet.

Como podemos ver nas figuras $3.45 \mathrm{a}, 3.45 \mathrm{~b}$ e $3.45 \mathrm{c}$, o resultado referente à aplicação da PML para o sistema de primeira ordem é superior aos decorrentes da aplicação das condições de Cerjan e de Higdon de primeira ordem, se assemelhando à condição de Higdon de segunda ordem, à A2 e às HABCs associadas a essas. A versão para a equação na forma de segunda ordem, cujos resultados se encontram nas imagens 3.46a, 3.46b e 3.46c, também apresenta desempenho satisfatório. A tabela de erros 3.5 permite verificar que a HABC modificada com a condição A2 e a PML para a equação na forma de segunda ordem apresentam os resultados mais favoráveis, seguidas da versão para o sistema de primeira ordem, a HABC original com a condição A2 e a HABC modificada com a condição de Higdon de segunda ordem.

Para a condição de Sochacki, temos resultados semelhantes para ambos os amortecedores apresentados. O resultado para o amortecedor cúbico para $t=0.3$ é superior aos demais, uma vez que as reflexões são menos visíveis nesse caso. Para $t=0.45$, a aplicação do amortecedor expoente resultou em reflexões mais acentuadas em relação aos demais amortecedores; de modo geral, no entanto, os três casos apresentaram resultados similares.

É importante observar que, em todos os casos apresentados, não foram observados problemas em relação aos cantos do domínio. Em particular, a condição A2 e as HABCs correspondentes apresentaram bons resultados mediante a aplicação da condição de compatibilidade sugerida.

\subsubsection{Desempenho das condições de contorno em relação à espessura da camada de amortecimento}

Nesta seção, apresentamos o desempenho das condições de Cerjan e Sochacki, das PMLs e das HABCs com o uso das condições A1, A2 e a condição de Higdon de segunda ordem em relação ao número de pontos da malha acrescentados a cada borda do domínio. Por simplicidade, a extensão do domínio será feita da mesma forma em todas as bordas e a espessura das camadas em pontos da malha será denotada por $L$. Para analisar os resultados, verificamo a tabela de erro referente a cada valor de $L$ para valores distintos de $t$, obtidas em comparação com a solução tomada como referência. 


\begin{tabular}{|c|c|c|c|c|c|c|c|}
\hline \multirow{2}{*}{ Método } & \multirow{2}{*}{$t$} & \multicolumn{3}{|c|}{ Erro relativo na norma infinito } & \multicolumn{3}{|c|}{ Erro absoluto na norma 2} \\
\hline & & $L=10$ & $L=20$ & $L=40$ & $L=10$ & $L=20$ & $L=40$ \\
\hline \multirow{3}{*}{ Cerjan } & 0.35 & 0.642 & 0.377 & 0.377 & $8.18 \cdot 10^{-9}$ & $6.29 \cdot 10^{-9}$ & $6.29 \cdot 10^{-9}$ \\
\hline & 0.4 & 0.785 & 0.453 & 0.453 & $2.49 \cdot 10^{-8}$ & $1.87 \cdot 10^{-8}$ & $1.87 \cdot 10^{-8}$ \\
\hline & 0.45 & 2.233 & 1.394 & 1.394 & $2.98 \cdot 10^{-8}$ & $2.41 \cdot 10^{-8}$ & $2.40 \cdot 10^{-8}$ \\
\hline \multirow{3}{*}{$\begin{array}{l}\text { HABC } 1 \\
\text { com A1 }\end{array}$} & 0.35 & 0.029 & 0.022 & 0.015 & $4.23 \cdot 10^{-10}$ & $3.35 \cdot 10^{-10}$ & $2.47 \cdot 10^{-10}$ \\
\hline & 0.4 & 0.084 & 0.067 & 0.049 & $1.27 \cdot 10^{-9}$ & $1.06 \cdot 10^{-9}$ & $8.20 \cdot 10^{-10}$ \\
\hline & 0.45 & 0.296 & 0.273 & 0.243 & $1.47 \cdot 10^{-9}$ & $1.49 \cdot 10^{-9}$ & $1.47 \cdot 10^{-9}$ \\
\hline \multirow{3}{*}{$\begin{array}{l}\text { HABC } 2 \\
\text { com A1 }\end{array}$} & 0.35 & 0.020 & 0.006 & $1.12 \cdot 10^{-4}$ & $3.14 \cdot 10^{-10}$ & $9.92 \cdot 10^{-11}$ & $1.41 \cdot 10^{-12}$ \\
\hline & 0.4 & 0.068 & 0.028 & 0.004 & $1.08 \cdot 10^{-9}$ & $5.26 \cdot 10^{-10}$ & $6.78 \cdot 10^{-11}$ \\
\hline & 0.45 & 0.341 & 0.265 & 0.030 & $1.80 \cdot 10^{-9}$ & $1.24 \cdot 10^{-9}$ & $2.84 \cdot 10^{-10}$ \\
\hline \multirow{3}{*}{$\begin{array}{l}\text { HABC } 1 \\
\text { com A2 }\end{array}$} & 0.35 & $8.20 \cdot 10^{-4}$ & $3.98 \cdot 10^{-4}$ & $1.98 \cdot 10^{-4}$ & $2.05 \cdot 10^{-11}$ & $1.00 \cdot 10^{-11}$ & $5.02 \cdot 10^{-12}$ \\
\hline & 0.4 & 0.003 & 0.001 & $6.75 \cdot 10^{-4}$ & $4.63 \cdot 10^{-11}$ & $2.28 \cdot 10^{-11}$ & $1.12 \cdot 10^{-11}$ \\
\hline & 0.45 & 0.024 & 0.015 & 0.008 & $8.02 \cdot 10^{-11}$ & $4.23 \cdot 10^{-11}$ & $2.09 \cdot 10^{-11}$ \\
\hline \multirow{3}{*}{$\begin{array}{l}\text { HABC } 2 \\
\text { com A2 }\end{array}$} & 0.35 & 0.001 & $3.39 \cdot 10^{-5}$ & $2.08 \cdot 10^{-6}$ & $1.95 \cdot 10^{-11}$ & $9.37 \cdot 10^{-13}$ & $4.27 \cdot 10^{-14}$ \\
\hline & 0.4 & 0.002 & $3.16 \cdot 10^{-4}$ & $1.33 \cdot 10^{-5}$ & $5.85 \cdot 10^{-11}$ & $6.19 \cdot 10^{-12}$ & $2.13 \cdot 10^{-13}$ \\
\hline & 0.45 & 0.036 & 0.007 & $1.50 \cdot 10^{-4}$ & $1.41 \cdot 10^{-10}$ & $2.92 \cdot 10^{-11}$ & $1.36 \cdot 10^{-12}$ \\
\hline \multirow{3}{*}{$\begin{array}{l}\text { HABC } 1 \\
\text { com } \\
\text { Higdon } 2\end{array}$} & 0.35 & 0.004 & 0.002 & 0.001 & $6.38 \cdot 10^{-11}$ & $3.99 \cdot 10^{-11}$ & $2.07 \cdot 10^{-11}$ \\
\hline & 0.4 & 0.007 & 0.004 & 0.002 & $1.42 \cdot 10^{-10}$ & $1.01 \cdot 10^{-10}$ & $6.00 \cdot 10^{-11}$ \\
\hline & 0.45 & 0.034 & 0.027 & 0.017 & $2.24 \cdot 10^{-10}$ & $1.76 \cdot 10^{-10}$ & $1.14 \cdot 10^{-10}$ \\
\hline \multirow{3}{*}{$\begin{array}{l}\text { HABC } 2 \\
\text { com } \\
\text { Higdon } 2\end{array}$} & 0.35 & 0.003 & $5.49 \cdot 10^{-4}$ & $8.83 \cdot 10^{-6}$ & $4.80 \cdot 10^{-11}$ & $7.66 \cdot 10^{-12}$ & $1.05 \cdot 10^{-13}$ \\
\hline & 0.4 & 0.006 & 0.002 & $1.74 \cdot 10^{-4}$ & $1.28 \cdot 10^{-10}$ & $5.38 \cdot 10^{-11}$ & $2.97 \cdot 10^{-12}$ \\
\hline & 0.45 & 0.035 & 0.010 & 0.002 & $2.15 \cdot 10^{-10}$ & $1.04 \cdot 10^{-10}$ & $1.81 \cdot 10^{-11}$ \\
\hline \multirow{3}{*}{ PML 1} & 0.35 & $7.15 \cdot 10^{-4}$ & $1.60 \cdot 10^{-4}$ & $2.37 \cdot 10^{-5}$ & $1.12 \cdot 10^{-11}$ & $2.67 \cdot 10^{-12}$ & $4.29 \cdot 10^{-13}$ \\
\hline & 0.4 & 0.040 & $7.10 \cdot 10^{-4}$ & $1.13 \cdot 10^{-4}$ & $3.82 \cdot 10^{-11}$ & $1.40 \cdot 10^{-11}$ & $2.27 \cdot 10^{-12}$ \\
\hline & 0.45 & 0.015 & 0.007 & 0.001 & $7.60 \cdot 10^{-11}$ & $3.69 \cdot 10^{-11}$ & $6.59 \cdot 10^{-12}$ \\
\hline \multirow{3}{*}{ PML 2} & 0.35 & $8.45 \cdot 10^{-4}$ & $1.45 \cdot 10^{-4}$ & $1.94 \cdot 10^{-5}$ & $1.45 \cdot 10^{-11}$ & $2.33 \cdot 10^{-12}$ & $3.30 \cdot 10^{-13}$ \\
\hline & 0.4 & 0.003 & $4.33 \cdot 10^{-4}$ & $6.05 \cdot 10^{-5}$ & $4.67 \cdot 10^{-11}$ & $8.47 \cdot 10^{-12}$ & $1.07 \cdot 10^{-12}$ \\
\hline & 0.45 & 0.028 & 0.002 & $3.12 \cdot 10^{-4}$ & $7.28 \cdot 10^{-11}$ & $1.19 \cdot 10^{-11}$ & $2.18 \cdot 10^{-12}$ \\
\hline S. & 0.35 & 0.221 & 0.077 & 0.012 & $5.20 \cdot 10^{-9}$ & $1.21 \cdot 10^{-9}$ & $1.72 \cdot 10^{-10}$ \\
\hline \multirow[t]{2}{*}{ Cúbico } & 0.4 & 0.269 & 0.129 & 0.053 & $1.05 \cdot 10^{-8}$ & $4.92 \cdot 10^{-9}$ & $1.78 \cdot 10^{-9}$ \\
\hline & 0.45 & 1.623 & 0.640 & 0.175 & $1.31 \cdot 10^{-8}$ & $6.30 \cdot 10^{-9}$ & $3.23 \cdot 10^{-9}$ \\
\hline \multirow{3}{*}{$\begin{array}{l}\text { S. } \\
\text { Expoente }\end{array}$} & 0.35 & 0.521 & 0.089 & 0.036 & $1.39 \cdot 10^{-8}$ & $2.82 \cdot 10^{-9}$ & $1.16 \cdot 10^{-9}$ \\
\hline & 0.4 & 0.596 & 0.116 & 0.048 & $1.80 \cdot 10^{-8}$ & $4.11 \cdot 10^{-9}$ & $1.81 \cdot 10^{-9}$ \\
\hline & 0.45 & 3.013 & 0.678 & 0.284 & $2.02 \cdot 10^{-8}$ & $5.78 \cdot 10^{-9}$ & $2.46 \cdot 10^{-9}$ \\
\hline \multirow{3}{*}{ S. Linear } & 0.35 & 0.164 & 0.074 & 0.043 & $4.07 \cdot 10^{-9}$ & $2.34 \cdot 10^{-9}$ & $1.33 \cdot 10^{-9}$ \\
\hline & 0.4 & 0.287 & 0.100 & 0.059 & $9.88 \cdot 10^{-9}$ & $3.91 \cdot 10^{-9}$ & $2.60 \cdot 10^{-9}$ \\
\hline & 0.45 & 1.067 & 0.719 & 0.440 & $1.24 \cdot 10^{-8}$ & $5.38 \cdot 10^{-9}$ & $3.44 \cdot 10^{-9}$ \\
\hline
\end{tabular}

Tabela 3.6: Erro para as camadas de amortecimento com diferentes valores de L.

Para a condição de Cerjan, podemos ver que o aumento no valor de $L$ de 10 para 20 resultou em uma melhora considerável nos resultados obtidos, mas os erros para $L=20$ e $L=40$ são bastante próximos. $\mathrm{O}$ valor de $\tilde{\lambda}$ utilizado foi determinado experimentalmente tendo como base testes para $L=20$, de modo que é possível considerar a busca por constantes que sejam mais adequadas para os demais valores de $L$. Já as HABCs e as PMLs apresentaram resultados superiores para camadas de amortecimento com mais pontos, assim como a condição de Sochacki com os amortecedores cúbico, expoente e linear. Esses resultados indicam que o aumento da camada de amortecimento, embora resulte em acréscimo no custo computacional da aplicação dos métodos, pode ser utilizada para redução das reflexões causadas pelo truncamento do domínio. 


\subsection{Equação da onda bidimensional - Exemplo 2}

No segundo exemplo, resolvemos a equação:

$$
\begin{aligned}
u_{t t}-\left(u_{x x}+u_{y y}\right) & =0,(x, y) \in[0,1] \times[0,1] \\
u(x, y, 0) & =e^{-0.5\left[(8 x-4)^{2}+(8 y-4)^{2}\right]}, \\
u_{t}(x, y, 0) & =0 .
\end{aligned}
$$

Neste exemplo, tomamos novamente condições iniciais não nulas e $f(x, y, t) \equiv 0$. Aqui, os efeitos do truncamento do domínio podem ser percebidos desde o início da simulação. Assim como nos exemplos anteriores, a primeira seção é dedicada à apresentação dos resultados gerais da aplicação de cada condição de contorno à resolução numérica da equação dada. Na segunda seção deste exemplo, abordamos os efeitos dos parâmetros escolhidos na eficácia das condições de Cerjan, Sochacki e da condição de Higdon de primeira ordem. As medidas de erro adotadas são:

$$
\left\|e^{n}\right\|_{\infty}=\sup _{i, j}\left\{\left|u_{i, j}^{*^{n}}-u_{i, j}^{n}\right|\right\},\left\|e^{n}\right\|_{2}=\left(\frac{\sum_{i=0}^{N} \sum_{j=0}^{N}\left|u_{i, j}^{*^{n}}-u_{i, j}^{n}\right|^{2}}{M}\right)^{\frac{1}{2}} .
$$

Para a obtenção dos resultados, foram utilizados:

- Tempo total de simulação: $T=1$;

- Malha de $201 \times 201$ pontos no espaço;

- $\Delta x=0.005, \Delta y=0.005, \Delta t=0.002, \lambda=c \frac{\Delta t}{\Delta x}=0.4$

Os parâmetros adotados para a aplicação dos métodos a esse exemplo são

- $L=20$, ou seja, $\delta=20 \cdot 0.005=0.1$;

- Cerjan: $\tilde{\lambda}=0.0001$;

- HABC 2: $M=2, \alpha=1.5+0.07(L-M)$;

- Condição de Higdon de primeira ordem: $\alpha=\frac{\pi}{8}, a=b=0.5$;

- Condição de Higdon de segunda ordem: $\alpha_{1}=0, \alpha_{2}=\frac{\pi}{4}, a_{1}=a_{2}=b_{1}=b_{2}=0.5$;

- PML para o sistema de primeira ordem: $R=10^{-4}$,

$$
d(x)=\left\{\begin{array}{l}
\frac{3}{40 \Delta x} \ln \left(\frac{1}{0.0001}\right)\left(\frac{x}{20 \Delta x}\right)^{2}, \text { se } x<0 \\
\frac{3}{40 \Delta x} \ln \left(\frac{1}{0.0001}\right)\left(\frac{x-1}{20 \Delta x}\right)^{2}, \text { se } x>1
\end{array}\right.
$$

- PML para a equação de segunda ordem: $R=10^{-2}$,

$$
d(x)=\left\{\begin{array}{l}
\frac{3}{40 \Delta x} \ln \left(\frac{1}{0.01}\right)\left(\frac{x}{20 \Delta x}\right)^{2}, \text { se } x<0 \\
\frac{3}{40 \Delta x} \ln \left(\frac{1}{0.01}\right)\left(\frac{x-1}{20 \Delta x}\right)^{2}, \text { se } x>1 ;
\end{array}\right.
$$

- Condição de Sochacki com amortecedor cúbico: $S=10^{9}$;

- Condição de Sochacki com amortecedor expoente: $S=10^{0.75}$;

- Condição de Sochacki com amortecedor linear: $S=10^{3.5}$. 


\subsubsection{Resultados gerais}

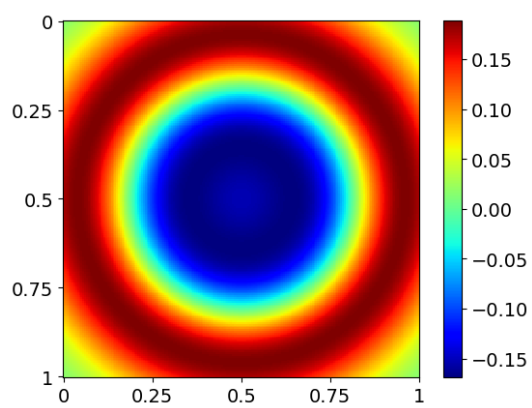

(a) $t=0.4$

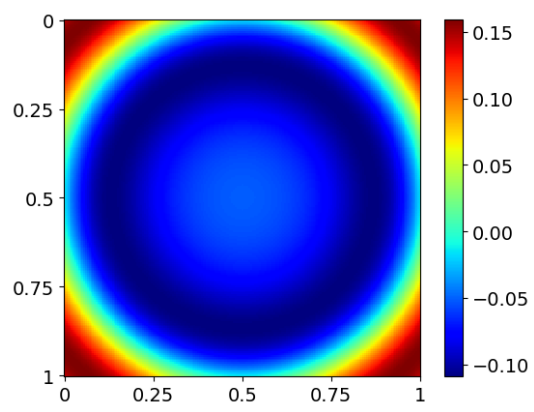

(b) $t=0.6$

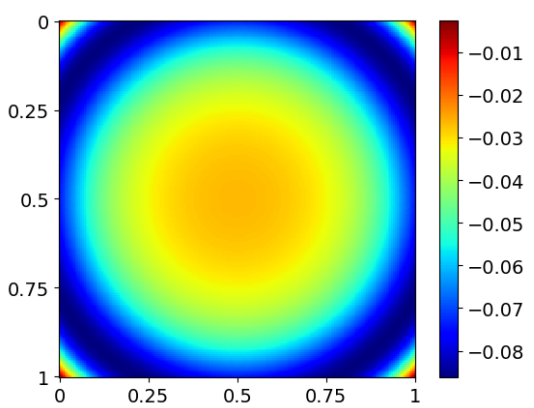

(c) $t=0.8$

Figura 3.50: Solução tomada como referência para o segundo exemplo bidimensional.

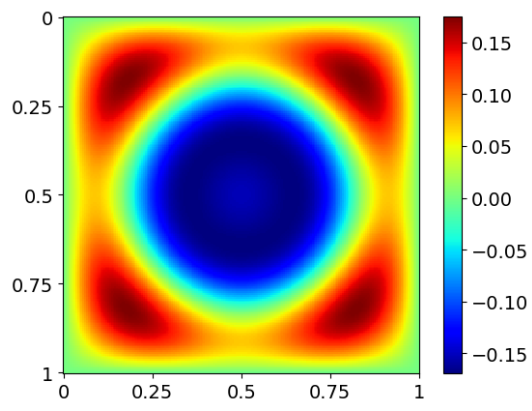

(a) $t=0.4$

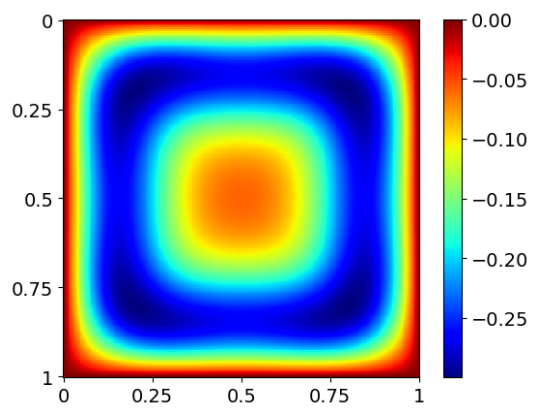

(b) $t=0.6$

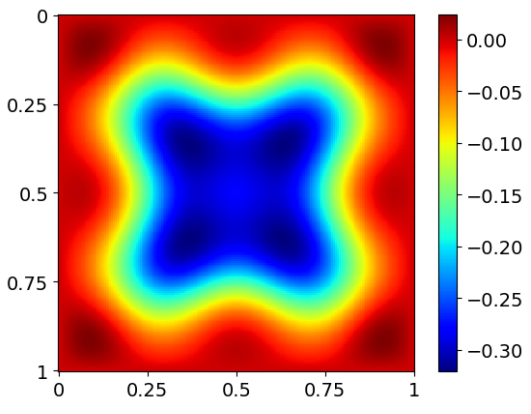

(c) $t=0.8$

Figura 3.51: Condição de Dirichlet $u=0$ aplicada ao segundo exemplo bidimensional.

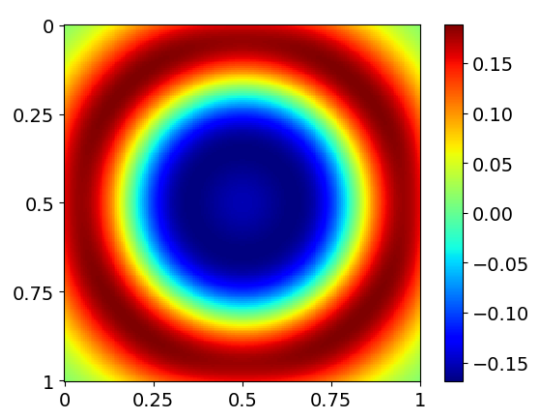

(a) $t=0.4$

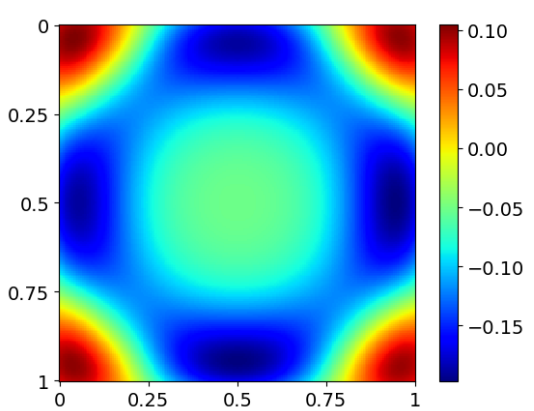

(b) $t=0.6$

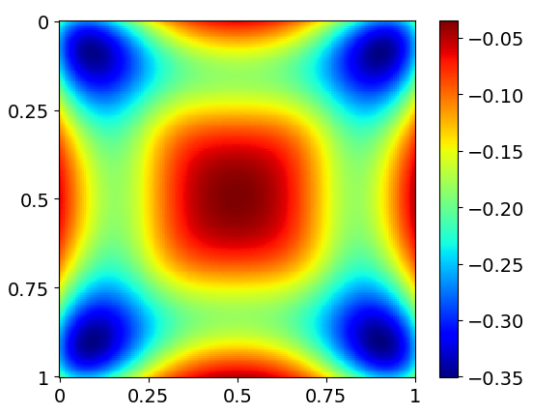

(c) $t=0.8$

Figura 3.52: Condição de Cerjan, com $\tilde{\lambda}=0.0001$, aplicada ao segundo exemplo bidimensional. 


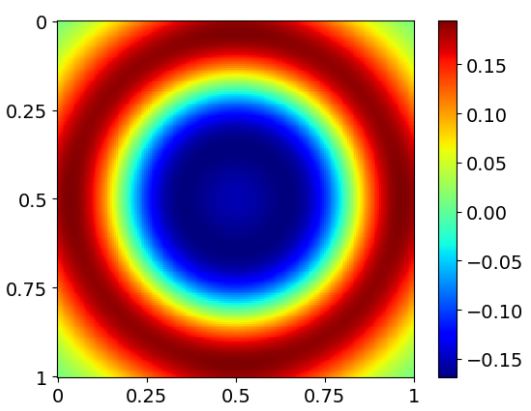

(a) $t=0.4$

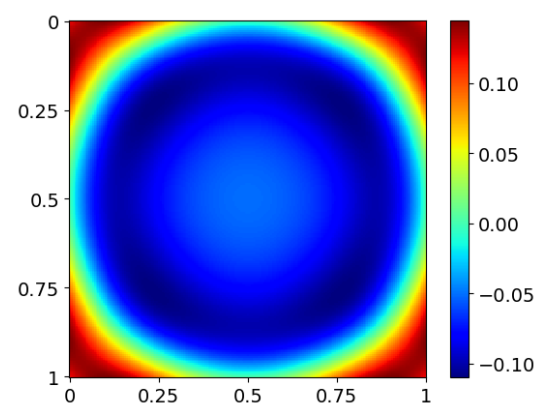

(b) $t=0.6$

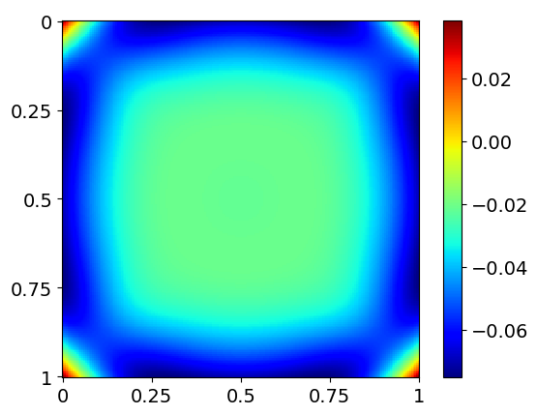

(c) $t=0.8$

Figura 3.53: Condição A1 aplicada ao segundo exemplo bidimensional.

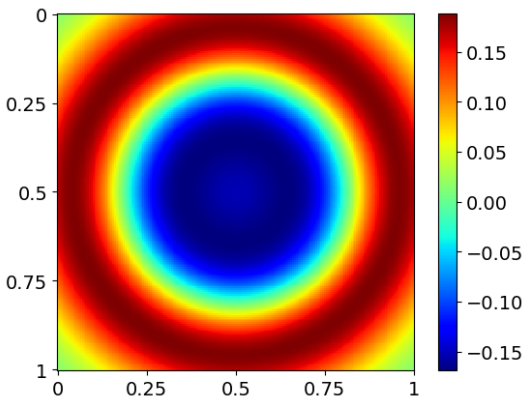

(a) $t=0.4$

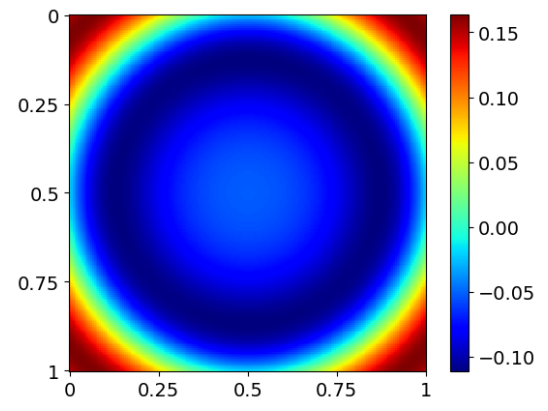

(b) $t=0.6$

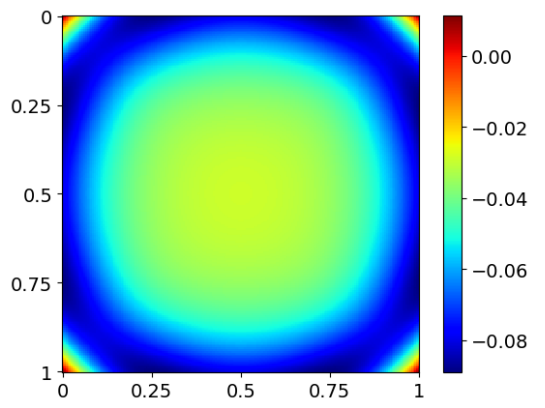

(c) $t=0.8$

Figura 3.54: Condição A2 aplicada ao segundo exemplo bidimensional.

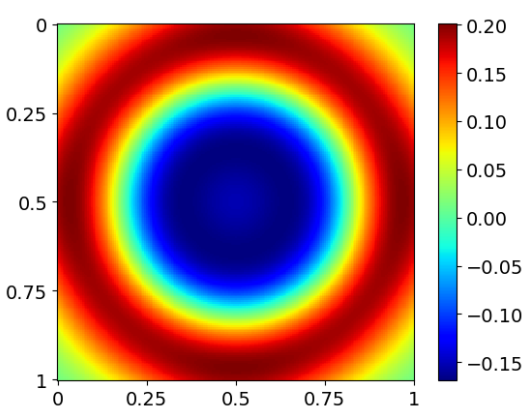

(a) $t=0.4$

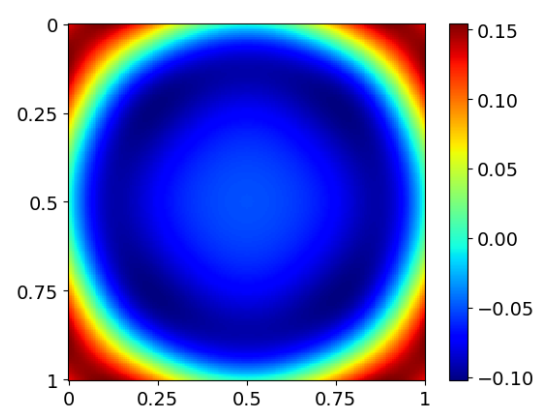

(b) $t=0.6$

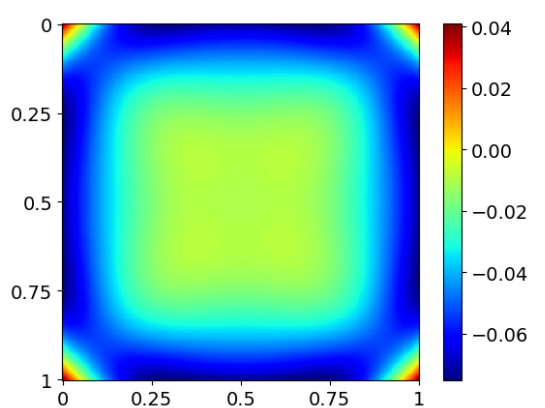

(c) $t=0.8$

Figura 3.55: Condição de Higdon de primeira ordem, com $\alpha=\frac{\pi}{8}, a=b=0.5$, aplicada ao segundo exemplo bidimensional. 


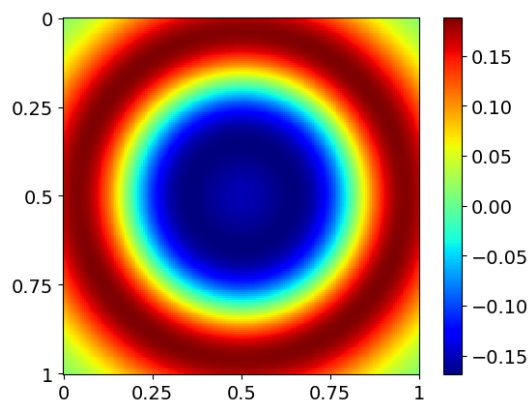

(a) $t=0.4$

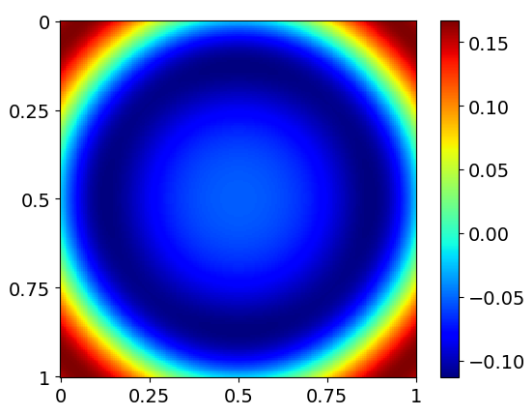

(b) $t=0.6$

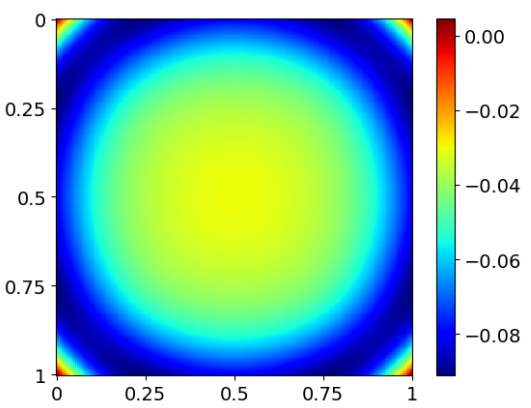

(c) $t=0.8$

Figura 3.56: Condição de Higdon de segunda ordem, com $\alpha_{1}=0, \alpha_{2}=\frac{\pi}{4}, a_{1}=a_{2}=b_{1}=b_{2}=0.5$, aplicada ao segundo exemplo bidimensional

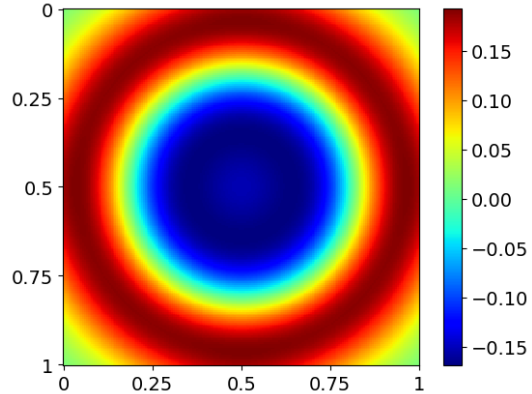

(a) $t=0.4$

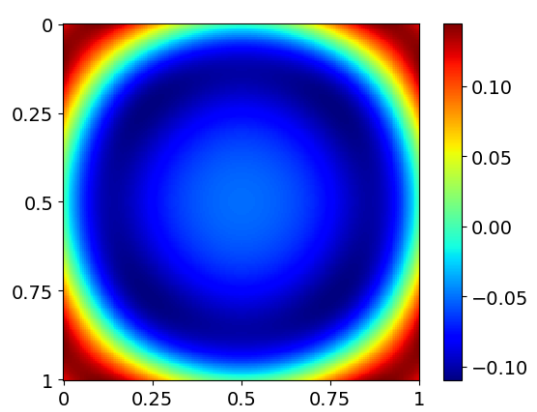

(b) $t=0.6$

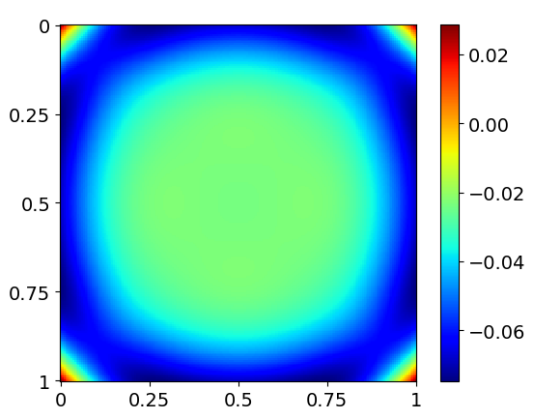

(c) $t=0.8$

Figura 3.57: HABC original com a condição A1 aplicada ao segundo exemplo bidimensional.

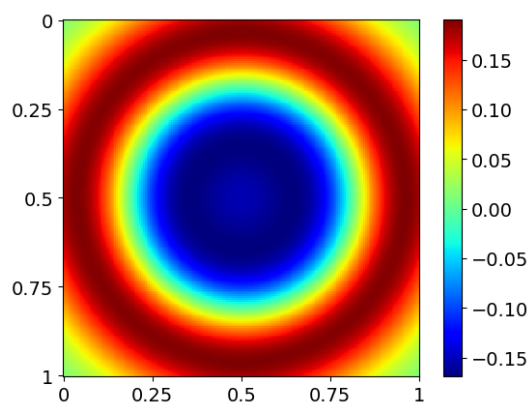

(a) $t=0.4$

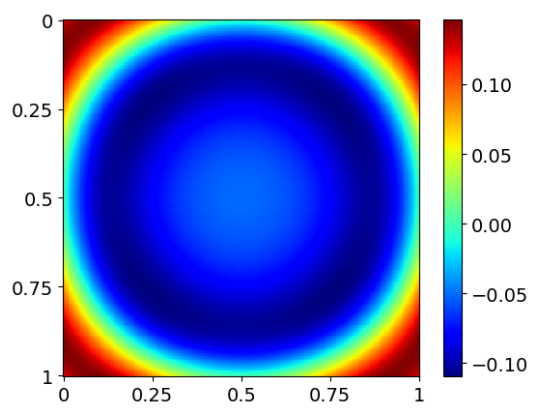

(b) $t=0.6$

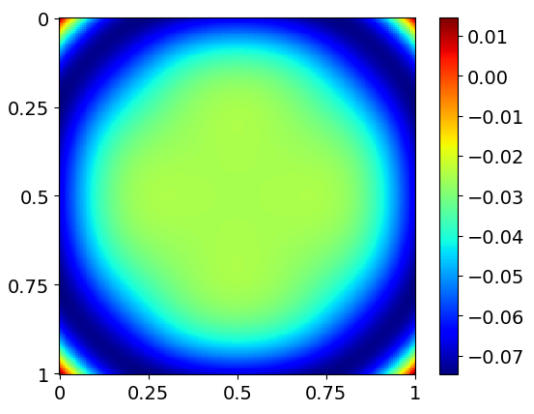

(c) $t=0.8$

Figura 3.58: $H A B C$ modificada com a condição A1, com $M=2$ e $\alpha=1.5+0.07(L-M)$, aplicada ao segundo exemplo bidimensional. 


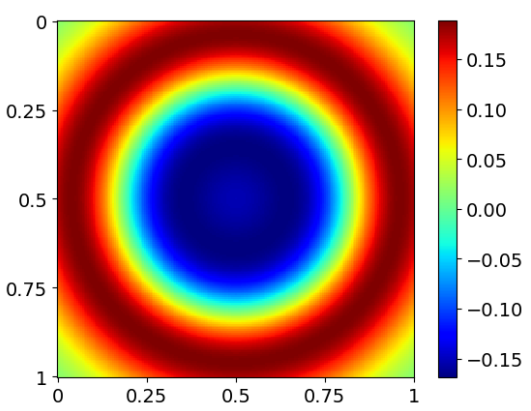

(a) $t=0.4$

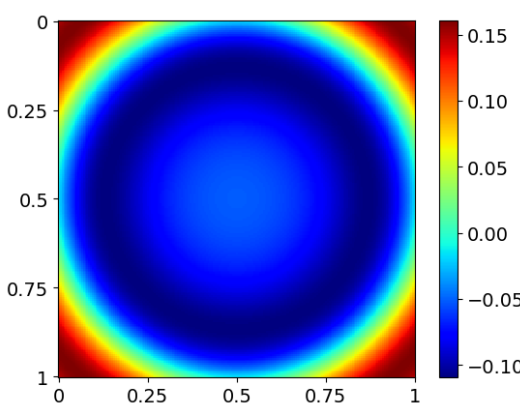

(b) $t=0.6$

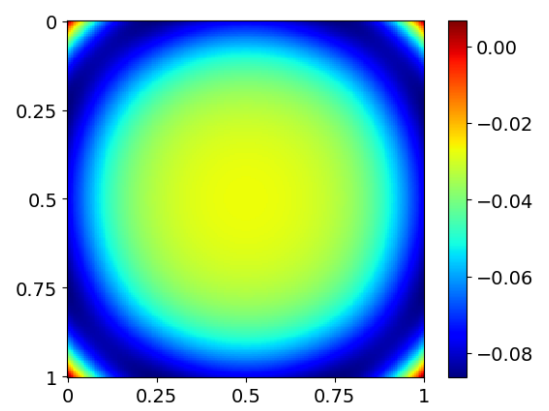

(c) $t=0.8$

Figura 3.59: HABC original com a condição A2 aplicada ao segundo exemplo bidimensional.

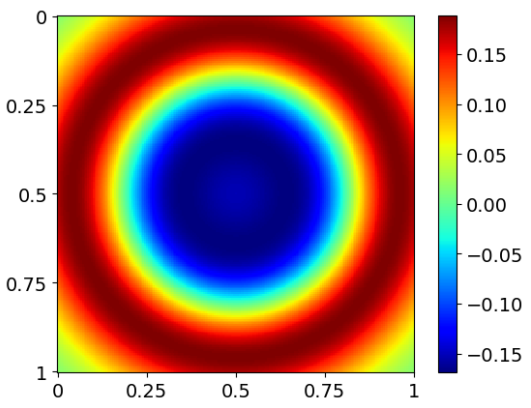

(a) $t=0.4$

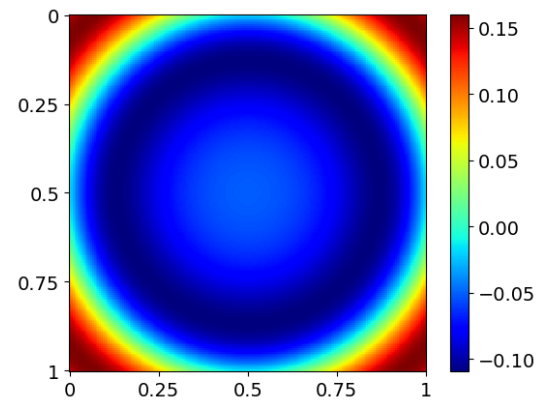

(b) $t=0.6$

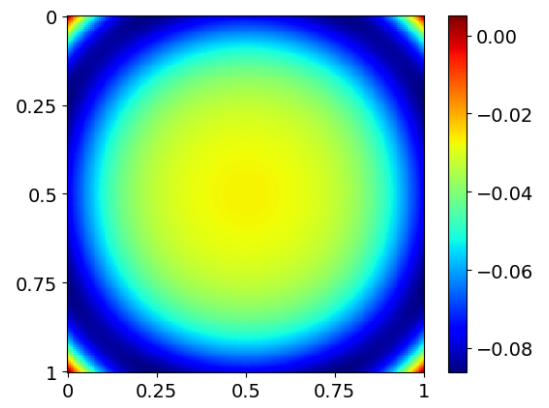

(c) $t=0.8$

Figura 3.60: $H A B C$ modificada com a condição A2, com $M=2$ e $\alpha=1.5+0.07(L-M)$, aplicada ao segundo exemplo bidimensional.

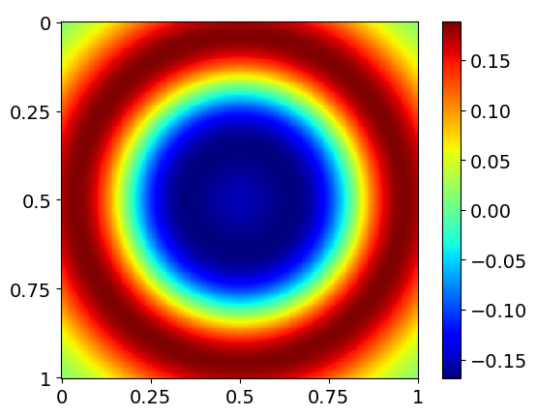

(a) $t=0.4$

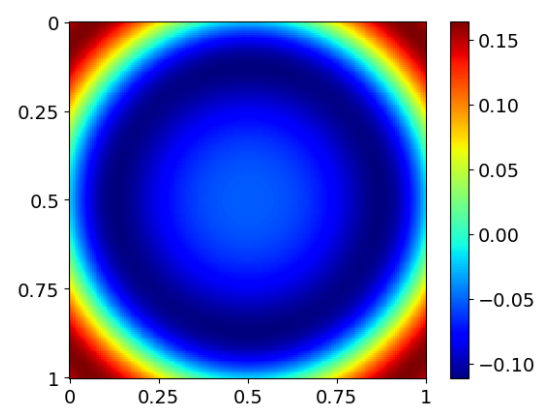

(b) $t=0.6$

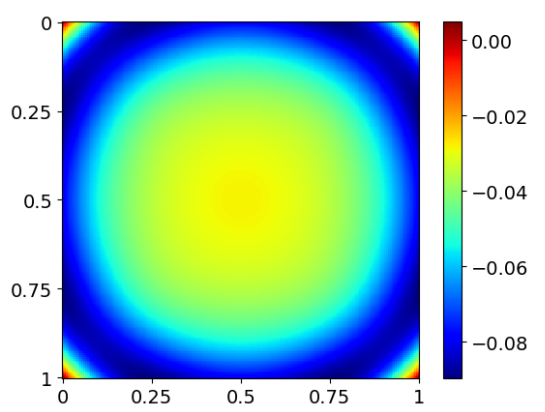

(c) $t=0.8$

Figura 3.61: HABC original com a condição de Higdon de segunda ordem, com $\alpha_{1}=0, \alpha_{2}=\frac{\pi}{4}$ e $a_{1}=$ $a_{2}=b_{1}=b_{2}=0.5$, aplicada ao segundo exemplo bidimensional. 


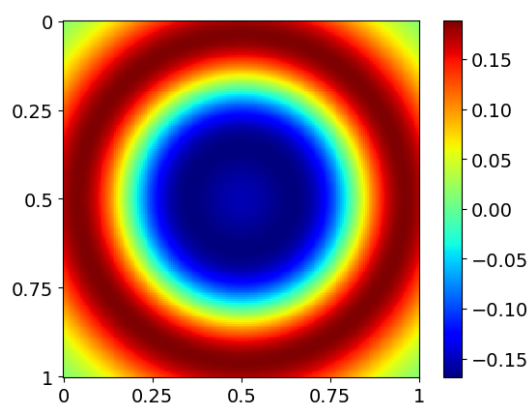

(a) $t=0.4$

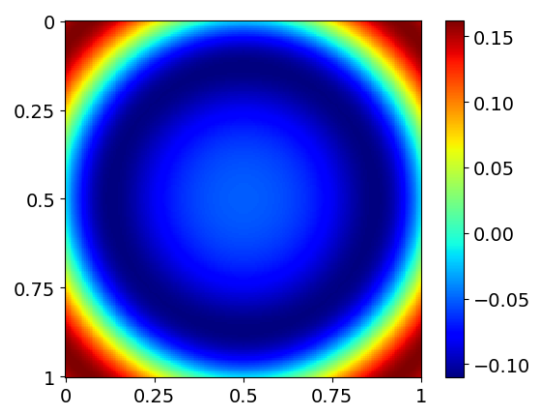

(b) $t=0.6$

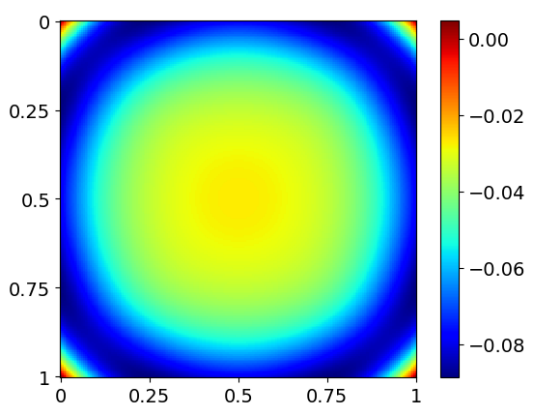

(c) $t=0.8$

Figura 3.62: $H A B C$ modificada com a condição de Higdon de segunda ordem, $M=2, \alpha=1.5+0.07(L-M)$, $\alpha_{1}=0, \alpha_{2}=\frac{\pi}{4}$ e $a_{1}=a_{2}=b_{1}=b_{2}=0.5$, aplicada ao segundo exemplo bidimensional.

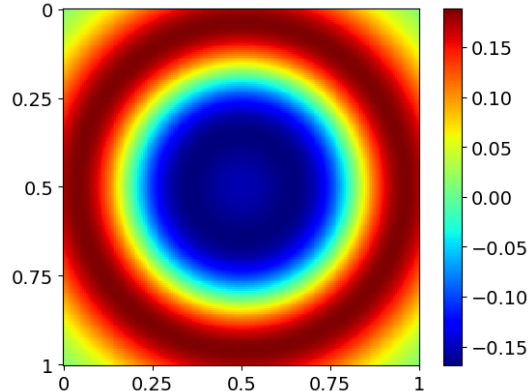

(a) $t=0.4$

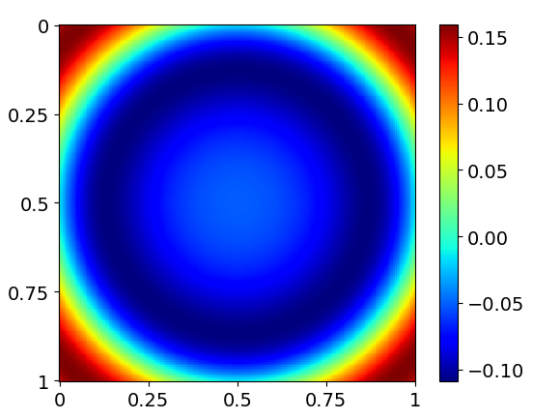

(b) $t=0.6$

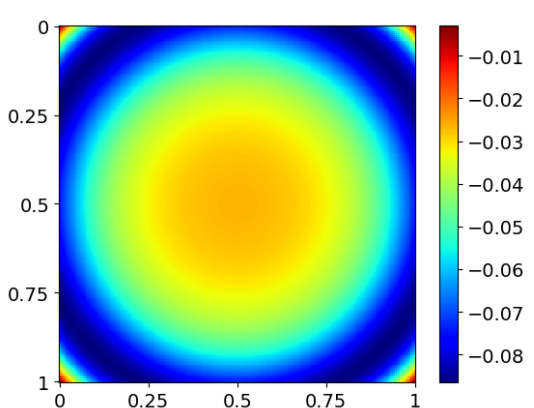

(c) $t=0.8$

Figura 3.63: Condição PML para o sistema de primeira ordem, com $R=10^{-4}$, aplicada ao segundo exemplo bidimensional.

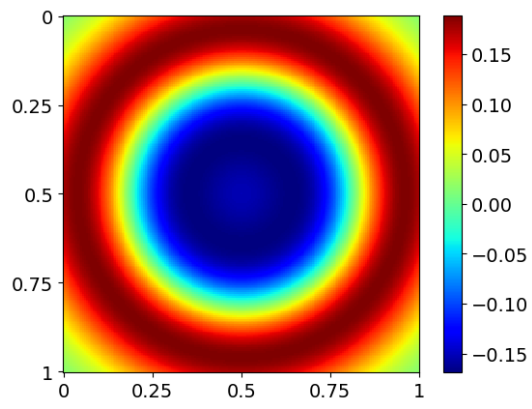

(a) $t=0.4$

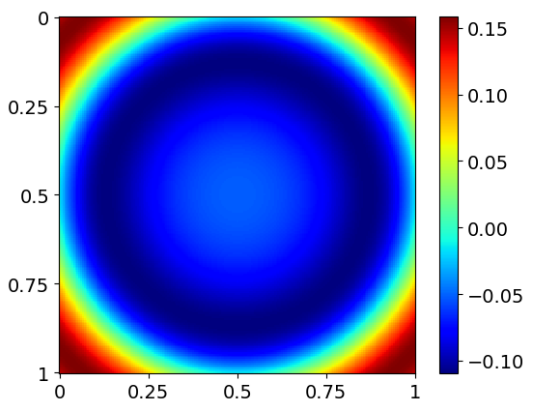

(b) $t=0.6$

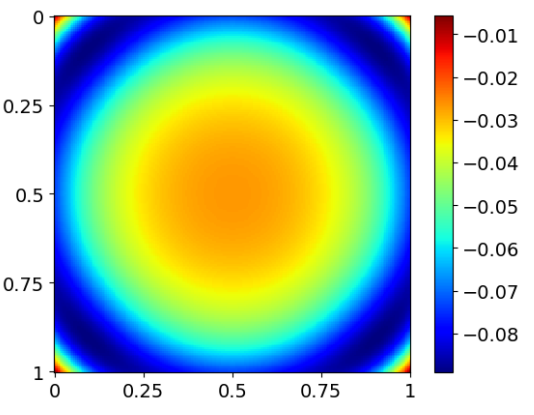

(c) $t=0.8$

Figura 3.64: Condição PML para a equação de segunda ordem, com $R=10^{-2}$, aplicada ao segundo exemplo bidimensional. 


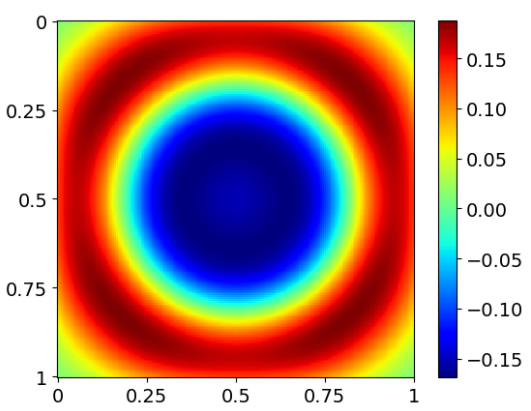

(a) $t=0.4$

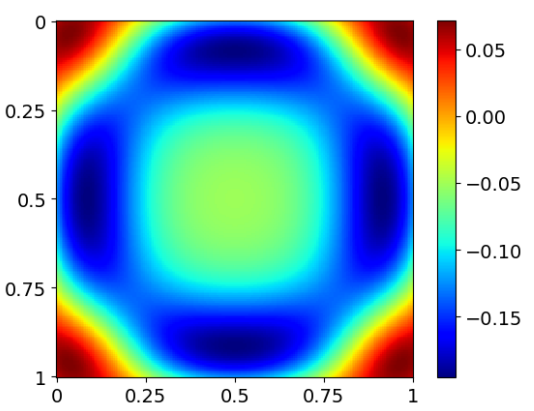

(b) $t=0.6$

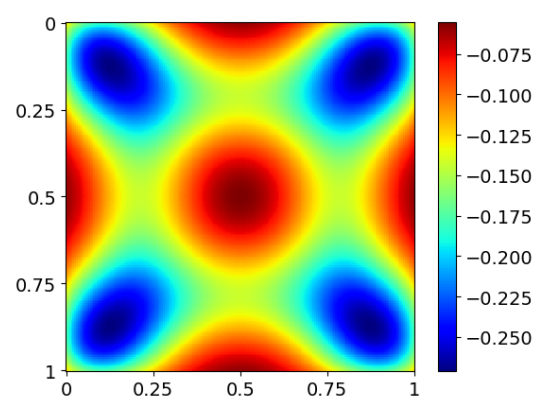

(c) $t=0.8$

Figura 3.65: Condição de Sochacki com amortecedor cúbico, com $S=10^{9}$, aplicada ao segundo exemplo bidimensional.

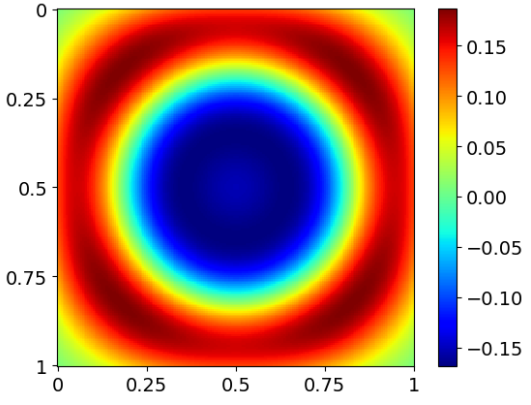

(a) $t=0.4$

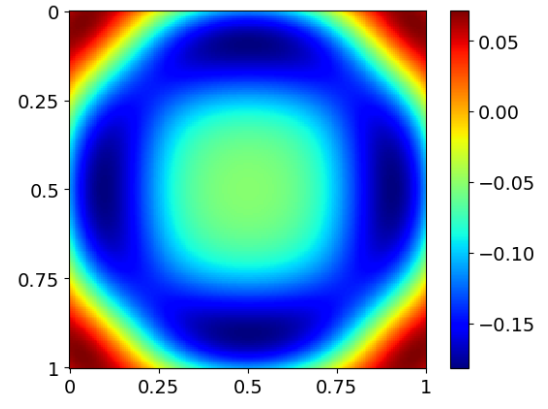

(b) $t=0.6$

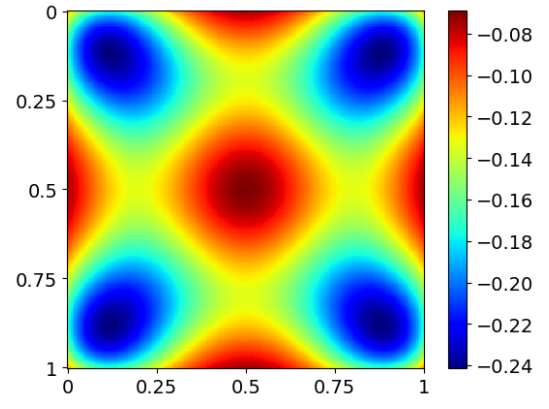

(c) $t=0.8$

Figura 3.66: Condição de Sochacki com amortecedor expoente, com $S=10^{0.75}$, aplicada ao segundo exemplo bidimensional.

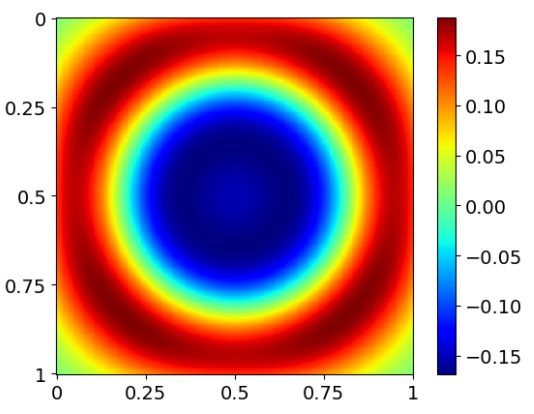

(a) $t=0.4$

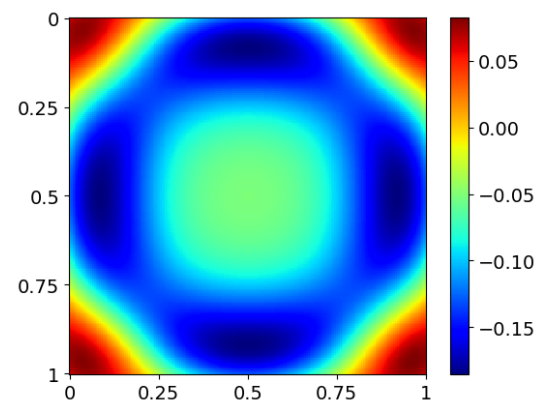

(b) $t=0.6$

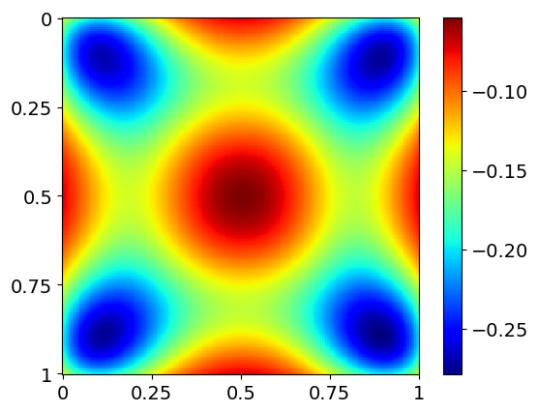

(c) $t=0.8$

Figura 3.67: Condição de Sochacki com amortecedor linear, com $S=10^{3.5}$, aplicada ao segundo exemplo bidimensional. 


\begin{tabular}{c|ccc|ccc} 
Método & \multicolumn{2}{|c|}{ Erro absoluto na norma infinito } & \multicolumn{3}{c}{ Erro absoluto na norma 2 } \\
& $t=0.4$ & $t=0.6$ & $t=0.8$ & $t=0.4$ & $t=0.6$ & $t=0.8$ \\
\hline Dirichlet & 0.1759 & 0.2404 & 0.2918 & 0.0611 & 0.1430 & 0.1430 \\
Cerjan & 0.0223 & 0.1542 & 0.2648 & 0.0040 & 0.0600 & 0.1421 \\
A1 & 0.0092 & 0.0268 & 0.0410 & 0.0021 & 0.0081 & 0.0154 \\
A2 & 0.0022 & 0.0056 & 0.0140 & $6.28 \cdot 10^{-4}$ & 0.0018 & 0.0039 \\
Higdon 1 & 0.0168 & 0.0170 & 0.0436 & 0.0041 & 0.0106 & 0.0191 \\
Higdon 2 & 0.0040 & 0.0092 & 0.0071 & $9.41 \cdot 10^{-4}$ & 0.0032 & 0.0046 \\
HABC 1 com A1 & 0.0064 & 0.0240 & 0.0312 & 0.0014 & 0.0070 & 0.0125 \\
HABC 2 com A1 & 0.0035 & 0.0187 & 0.0171 & $6.77 \cdot 10^{-4}$ & 0.0052 & 0.0089 \\
HABC 1 com A2 & $3.02 \cdot 10^{-4}$ & 0.0019 & 0.0092 & $8.07 \cdot 10^{-5}$ & $4.10 \cdot 10^{-4}$ & 0.0018 \\
HABC 2 com A2 & $1.54 \cdot 10^{-4}$ & $9.10 \cdot 10^{-4}$ & 0.0077 & $4.08 \cdot 10^{-5}$ & $2.19 \cdot 10^{-4}$ & 0.0014 \\
HABC 1 com Higdon 2 & 0.0019 & 0.0058 & 0.0075 & $4.17 \cdot 10^{-4}$ & 0.0019 & 0.0031 \\
HABC 2 com Higdon 2 & $7.70 \cdot 10^{-4}$ & 0.0036 & 0.0073 & $1.53 \cdot 10^{-4}$ & 0.0011 & 0.0023 \\
PML 1 & $3.48 \cdot 10^{-5}$ & $8.15 \cdot 10^{-5}$ & $4.14 \cdot 10^{-4}$ & $8.62 \cdot 10^{-6}$ & $3.16 \cdot 10^{-5}$ & $9.41 \cdot 10^{-5}$ \\
PML 2 & $1.76 \cdot 10^{-4}$ & 0.0012 & 0.0031 & $3.47 \cdot 10^{-5}$ & $5.49 \cdot 10^{-4}$ & 0.0013 \\
S. Cúbico & 0.0414 & 0.1152 & 0.1979 & 0.0098 & 0.0670 & 0.1150 \\
S. Expoente & 0.0442 & 0.0986 & 0.1663 & 0.0121 & 0.0620 & 0.1059 \\
S. Linear & 0.0375 & 0.1162 & 0.1966 & 0.0089 & 0.0610 & 0.1161
\end{tabular}

Tabela 3.7: Erros para o segundo teste bidimensional.

Os resultados apresentados para a condição de Cerjan mostram que a aplicação dessa condição de contorno não foi eficaz na simulação da propagação da onda em um domínio ilimitado, uma vez que as reflexões são perceptíveis. Comportamentos semelhantes são verificados para as condições de Sochacki e, a partir da tabela 3.7, vemos que os erros resultantes das aplicações de ambas as condições de contorno são consideravelmente próximos, embora a condição de Sochacki apresente desempenho ligeiramente superior.

Graficamente, os resultados das aplicações da condição A1 e da HABC original associada a essa são semelhantes, embora a tabela de erros indique superioridade da HABC neste caso. Ambos os resultados são mais favoráveis do que os referentes às condições de Cerjan e Sochacki; a solução decorrente da aplicação da HABC modificada associada à condição A1, por sua vez, se assemelha mais à solução de referência do que as obtidas a partir das condições mencionadas anteriormente.

Apesar de apresentar melhor atenuação das ondas incidentes do que as condições de Cerjan, Sochacki, a condição A1 e as HABCs associadas, a aplicação da condição A2 teve desempenho inferior às condições HABCs com a própria A2, sendo a HABC modificada a melhor opção entre as duas formas abordadas. O mesmo ocorre para a condição de Higdon de segunda ordem: sua aplicação como condição de absorção apresenta desempenho adequado, mas seu uso nas HABCs gera soluções mais próximas da solução de referência. A condição de Higdon de segunda ordem ainda é superior à condição de primeira ordem, cujos resultados, embora não superem os decorrentes das HABCs já mencionadas e da condição A2, se assemelha, em termos dos erros apresentados na tabela 3.7, à condição A1.

Por fim, a aplicação de ambas as PMLs resultou em amortecimento das ondas nos contornos, fornecendo as soluções graficamente mais próximas da solução de referência. A tabela de erros apresentada ainda indica que a PML para o sistema de primeira ordem foi o método cuja aplicação gerou os resultados mais favoráveis, seguida da PML para a equação na forma de segunda ordem.

Na próxima seção, apresentamos os erros obtidos para as condições de Cerjan, Sochacki e Higdon de primeira ordem, para diferentes escolhas de $\tilde{\lambda}, S$ e $\alpha$, respectivamente. 


\subsubsection{Desempenho das condições de Cerjan, Sochacki e Higdon de primeira ordem em relação aos parâmetros}

\begin{tabular}{c|cccc|cccc} 
& \multicolumn{4}{|c|}{ Erro absoluto na norma infinito } & \multicolumn{4}{|c}{ Erro absoluto na norma 2} \\
& $\tilde{\lambda}=10^{-4}$ & $\tilde{\lambda}=10^{-3}$ & $\tilde{\lambda}=10^{-2}$ & $\tilde{\lambda}=10^{-1}$ & $\tilde{\lambda}=10^{-4}$ & $\tilde{\lambda}=10^{-3}$ & $\tilde{\lambda}=10^{-2}$ & $\tilde{\lambda}=10^{-1}$ \\
\hline 0.4 & 0.0223 & 0.0238 & 0.0880 & 0.1522 & 0.0040 & 0.0044 & 0.0204 & 0.0441 \\
0.6 & 0.1542 & 0.1551 & 0.1656 & 0.2150 & 0.0600 & 0.0623 & 0.1083 & 0.1337 \\
0.8 & 0.2648 & 0.2652 & 0.2754 & 0.2805 & 0.1421 & 0.1428 & 0.1445 & 0.1406 \\
1 & 0.2703 & 0.2749 & 0.4784 & 0.4826 & 0.1377 & 0.1388 & 0.1670 & 0.1612
\end{tabular}

Tabela 3.8: Erro para a condição de Cerjan com diversos valores de $\tilde{\lambda}$.

\begin{tabular}{c|ccccc|ccccc} 
& \multicolumn{5}{|c|}{ Erro absoluto na norma infinito } & \multicolumn{4}{c}{ Erro absoluto na norma 2} \\
$t$ & $10^{7}$ & $10^{7.5}$ & $10^{8}$ & $10^{8.5}$ & $10^{9}$ & $10^{7}$ & $10^{7.5}$ & $10^{8}$ & $10^{8.5}$ & $10^{9}$ \\
\hline 0.4 & 0.0189 & 0.0197 & 0.0221 & 0.0284 & 0.0414 & 0.0037 & 0.0039 & 0.0045 & 0.0062 & 0.0098 \\
0.6 & 0.1488 & 0.1470 & 0.1417 & 0.1293 & 0.1153 & 0.0574 & 0.0575 & 0.0578 & 0.0597 & 0.0670 \\
0.8 & 0.2601 & 0.2563 & 0.2452 & 0.2204 & 0.1979 & 0.1405 & 0.1392 & 0.1354 & 0.1264 & 0.1150 \\
1 & 0.2648 & 0.2622 & 0.2555 & 0.2534 & 0.2906 & 0.1359 & 0.1347 & 0.1312 & 0.1230 & 0.1143
\end{tabular}

Tabela 3.9: Erro para a condição de Sochacki com amortecedor cúbico com diversos valores de $S$.

\begin{tabular}{c|ccccc|ccccc} 
& \multicolumn{5}{|c|}{ Erro absoluto na norma infinito } & \multicolumn{5}{c}{ Erro absoluto na norma 2} \\
$t$ & $10^{0.25}$ & $10^{0.5}$ & $10^{0.75}$ & 10 & $10^{1.25}$ & $10^{0.25}$ & $10^{0.5}$ & $10^{0.75}$ & 10 & $10^{1.25}$ \\
\hline 0.4 & 0.0270 & 0.0336 & 0.0442 & 0.0598 & 0.0796 & 0.0063 & 0.0085 & 0.0121 & 0.0175 & 0.0244 \\
0.6 & 0.1307 & 0.1171 & 0.0986 & 0.1065 & 0.1304 & 0.0576 & 0.0587 & 0.0620 & 0.0693 & 0.0811 \\
0.8 & 0.2222 & 0.1964 & 0.1663 & 0.1518 & 0.1648 & 0.1275 & 0.1182 & 0.1059 & 0.0946 & 0.0914 \\
1 & 0.2454 & 0.2431 & 0.2521 & 0.2689 & 0.2952 & 0.1243 & 0.1163 & 0.1061 & 0.0974 & 0.0968
\end{tabular}

Tabela 3.10: Erro para a condição de Sochacki com amortecedor expoente com diversos valores de $S$.

\begin{tabular}{c|ccccc|ccccc} 
& \multicolumn{5}{|c|}{ Erro absoluto na norma infinito } & \multicolumn{5}{c}{ Erro absoluto na norma 2} \\
$t$ & $10^{2.5}$ & $10^{2.75}$ & $10^{3}$ & $10^{3.25}$ & $10^{3.5}$ & $10^{2.5}$ & $10^{2.75}$ & $10^{3}$ & $10^{3.25}$ & $10^{3.5}$ \\
\hline 0.4 & 0.0209 & 0.0226 & 0.0255 & 0.0303 & 0.0375 & 0.0042 & 0.0047 & 0.0055 & 0.0068 & 0.0089 \\
0.6 & 0.1451 & 0.1418 & 0.1363 & 0.1279 & 0.1162 & 0.0575 & 0.0576 & 0.0579 & 0.0588 & 0.0610 \\
0.8 & 0.2524 & 0.2454 & 0.2341 & 0.2174 & 0.1966 & 0.1375 & 0.1350 & 0.1308 & 0.1245 & 0.1161 \\
1 & 0.2604 & 0.2567 & 0.2518 & 0.2493 & 0.2584 & 0.1332 & 0.1309 & 0.1272 & 0.1216 & 0.1145
\end{tabular}

Tabela 3.11: Erro para a condição de Sochacki com amortecedor linear com diversos valores de $S$.

\begin{tabular}{c|ccccc|ccccc} 
& \multicolumn{5}{|c|}{ Erro absoluto na norma infinito } & \multicolumn{5}{c}{ Erro absoluto na norma 2} \\
& $\alpha=\frac{\pi}{4}$ & $\alpha=\frac{\pi}{8}$ & $\alpha=\frac{\pi}{16}$ & $\alpha=\frac{\pi}{32}$ & $\alpha=\frac{\pi}{64}$ & $\alpha=\frac{\pi}{4}$ & $\alpha=\frac{\pi}{8}$ & $\alpha=\frac{\pi}{16}$ & $\alpha=\frac{\pi}{32}$ & $\alpha=\frac{\pi}{64}$ \\
\hline 0.4 & 0.0404 & 0.0168 & 0.0114 & 0.0101 & 0.0098 & 0.0125 & 0.0041 & 0.0026 & 0.0023 & 0.0023 \\
0.6 & 0.0383 & 0.0170 & 0.0244 & 0.0264 & 0.0269 & 0.0287 & 0.0106 & 0.0084 & 0.0082 & 0.0082 \\
0.8 & 0.0599 & 0.0436 & 0.0428 & 0.0426 & 0.0425 & 0.0351 & 0.0191 & 0.0165 & 0.0160 & 0.0159 \\
1 & 0.0863 & 0.0393 & 0.0338 & 0.0345 & 0.0347 & 0.0315 & 0.0204 & 0.0194 & 0.0193 & 0.0193
\end{tabular}

Tabela 3.12: Erro para a condição de Higdon de primeira ordem com diversos valores de $\alpha$.

A mudança nos valores de $\tilde{\lambda}$ gera diferenças consideráveis nos erros gerados pela aplicação da condição de Cerjan para este exemplo para alguns valores de $t$. Nesse caso, os valores mais altos de $\tilde{\lambda}$ resultaram em erros maiores, refletindo a sensibilidade do método à constante escolhida. 
A aplicação da condição de Sochacki com amortecedor cúbico com diversos valores de $S$, por sua vez, não gerou resultados tão distintos entre si quanto no caso da condição de Cerjan. É importante observar, no entanto, que existem diferenças consideráveis entre os erros obtidos com $S=10^{7}$ e $S=10^{9}$, de modo que, para este exemplo, a escolha de $S$ afetou o resultado obtido no uso da condição referida. As diferenças entre os erros obtidos para os valores de $S$ distintos também se verificam para os demais amortecedores, embora sejam menos significativas para o amortecedor linear.

Por fim, a condição de Higdon também apresentou sensibilidade em relação ao valor de $\alpha$. Como podemos ver, os erros para $\alpha=\frac{\pi}{4}$ são consideravelmente mais altos do que para os demais valores de $\alpha$ para este exemplo, que, por sua vez, não resultaram em erros tão discrepantes entre si. Assim, concluímos que o desempenho da condição de Higdon de primeira ordem, assim como das condições de Cerjan e Sochacki, foi influenciado pela escolha do parâmetro envolvido para este exemplo.

\subsection{Equação da onda bidimensional - Exemplo 3}

Neste exemplo, apresentamos simulações para a solução da seguinte equação:

$$
\begin{aligned}
u_{t t}-c^{2}(y)\left(u_{x x}+u_{y y}\right) & =f(t) \delta\left(\mathbf{x}-\mathbf{x}_{0}\right),(x, y) \in[0,1000] \times[0,1000] \\
u(x, y, 0) & =0, \\
u_{t}(x, y, 0) & =0,
\end{aligned}
$$

onde

$$
\begin{array}{r}
f(t)=\left[1-2 \cdot 0.010^{2} \pi^{2}\left(t-\frac{1}{0.010}\right)^{2}\right] e^{-0.010^{2} \pi^{2}\left(t-\frac{1}{0.010}\right)^{2}}, \\
\delta(\mathbf{x})=\left\{\begin{array}{l}
1, \text { se } \mathbf{x}=0, \\
0, \text { caso contrário }
\end{array} \quad, \mathbf{x}_{0}=(500,10)\right.
\end{array}
$$

e

$$
c(y)=\left\{\begin{array}{l}
1.5, \text { se } y<500, \\
2.5, \text { caso contrário. }
\end{array}\right.
$$

A representação gráfica da fonte $f(t)$ é a seguinte:

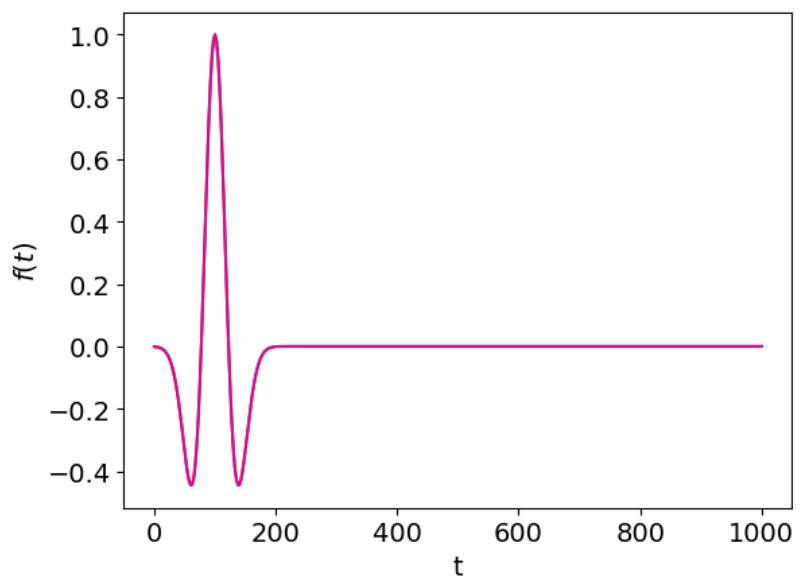

Figura 3.68: Gráfico de $f(t)$

Este exemplo representa a propagação de uma onda em meio bidimensional, decorrente da atuação de uma fonte no ponto $(500,10)$. Para esse teste, adotamos 
- Tempo total de simulação: $T=1000$

- Malha de $100 \times 100$ pontos no espaço

- $\Delta x=10, \Delta y=10, \Delta t=2.5$.

Nesse caso, avaliamos o desempenho apenas das condições de contorno que apresentaram os melhores resultados nos exemplos anteriores: A2, HABC modificada, condição de Higdon de segunda ordem e as PMLs. Tais condições são aplicadas apenas nos contornos laterais e no contorno inferior, sendo que, no superior, é utilizada uma condição de Dirichlet do tipo $u=0$. Os parâmetros adotados foram

- $L=20$, de modo que a espessura da camada de amortecimento é $\delta=200$;

- HABC modificada: $M=2, \alpha=1.5+0.07(L-M)$;

- Condição de Higdon de segunda ordem: $\alpha_{1}=0, \alpha_{2}=\frac{\pi}{4}, a_{1}=a_{2}=b_{1}=b_{2}=0.5$;

- PML para o sistema de primeira ordem: $R=10^{-4}$,

$$
d(x)=\left\{\begin{array}{l}
\frac{3}{40 \Delta x} \ln \left(\frac{1}{0.0001}\right)\left(\frac{x}{20 \Delta x}\right)^{2}, \text { se } x<0 \\
\frac{3}{40 \Delta x} \ln \left(\frac{1}{0.0001}\right)\left(\frac{x-1000}{20 \Delta x}\right)^{2}, \text { se } x>1000
\end{array}\right.
$$

- PML para a equação de segunda ordem: $R=10^{-7}$,

$$
d(x)=\left\{\begin{array}{l}
\frac{3}{40 \Delta x} \ln \left(\frac{1}{10^{-7}}\right)\left(\frac{x}{20 \Delta x}\right)^{2}, \text { se } x<0 \\
\frac{3}{40 \Delta x} \ln \left(\frac{1}{10^{-7}}\right)\left(\frac{x-1000}{20 \Delta x}\right)^{2}, \text { se } x>1000
\end{array}\right.
$$

Por fim, as medidas de erro adotadas foram:

$$
\frac{\left\|e^{n}\right\|_{\infty}}{\left\|u^{n}\right\|_{\infty}}=\frac{\sup _{i, j}\left\{\left|u_{i, j}^{* n}-u_{i, j}^{n}\right|\right\}}{\sup _{i, j}\left\{\left|u_{i, j}^{*^{n}}\right|\right\}},\left\|e^{n}\right\|_{2}=\left(\frac{\sum_{i=0}^{N} \sum_{j=0}^{N}\left|u_{i, j}^{*^{n}}-u_{i, j}^{n}\right|^{2}}{M}\right)^{\frac{1}{2}},
$$

com $M$ sendo o número de pontos da malha.

\subsubsection{Resultados}

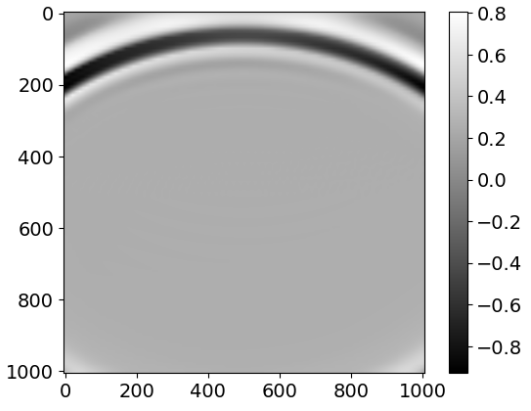

(a) $t=750$

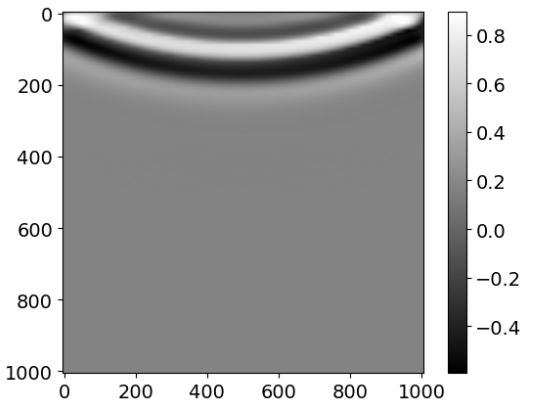

(b) $t=875$

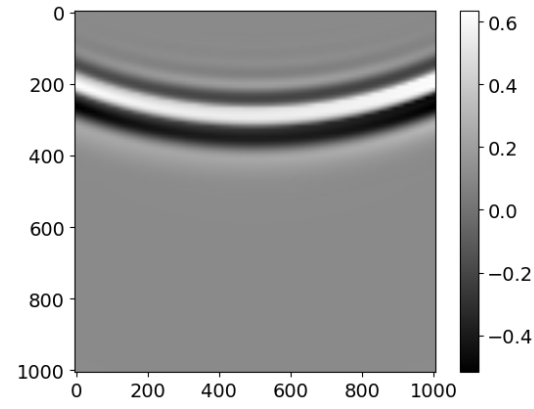

(c) $t=1000$

Figura 3.69: Solução tomada como referência para o terceiro exemplo bidimensional. 


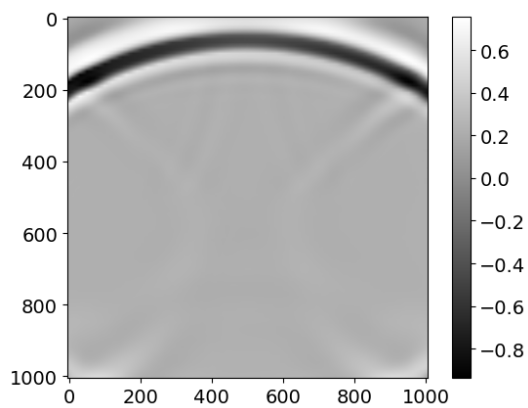

(a) $t=750$

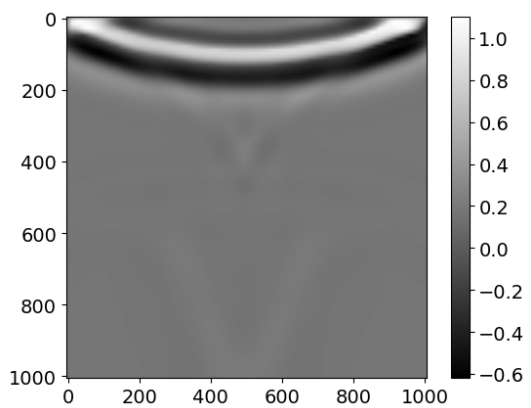

(b) $t=875$

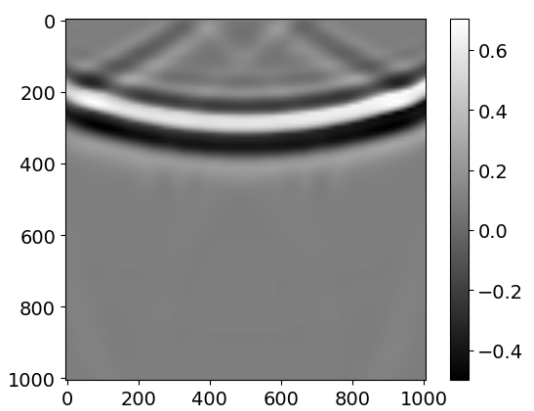

(c) $t=1000$

Figura 3.70: Condição A2 aplicada ao terceiro exemplo bidimensional.

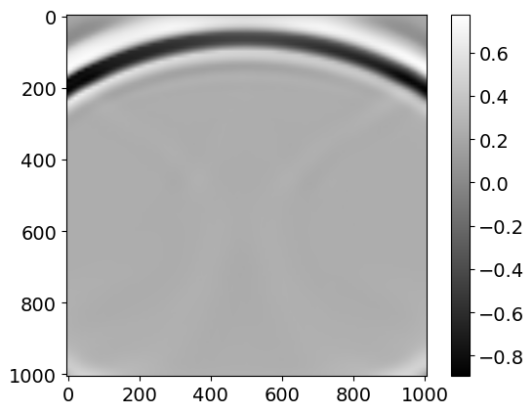

(a) $t=750$

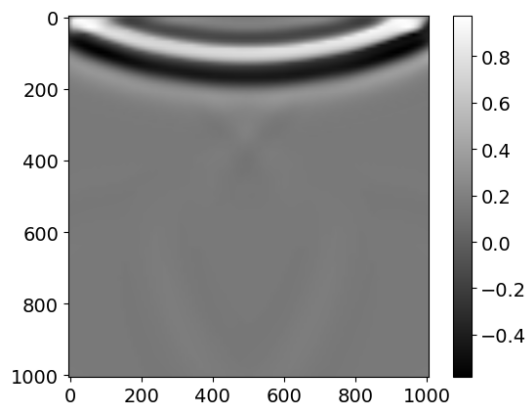

(b) $t=875$

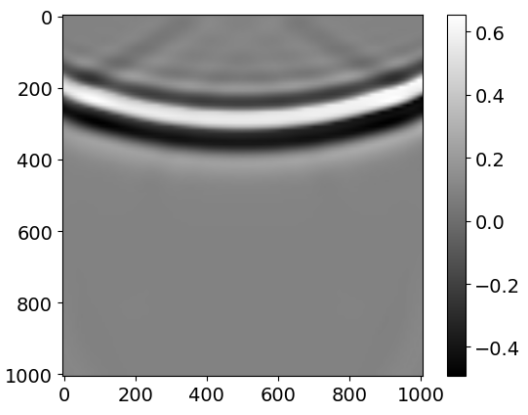

(c) $t=1000$

Figura 3.71: Condição de Higdon de segunda ordem, com $\alpha_{1}=0, \alpha_{2}=\frac{\pi}{4}, a_{1}=a_{2}=b_{1}=b_{2}=0.5$, aplicada ao terceiro exemplo bidimensional.

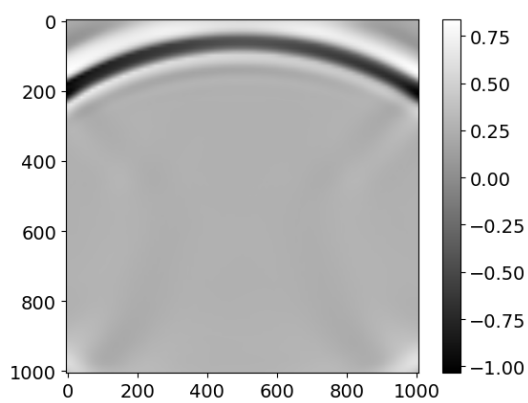

(a) $t=750$

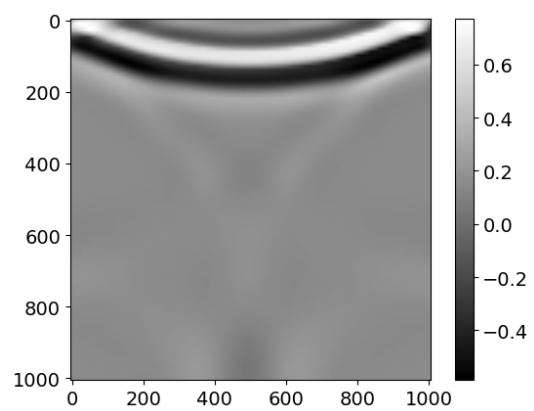

(b) $t=875$

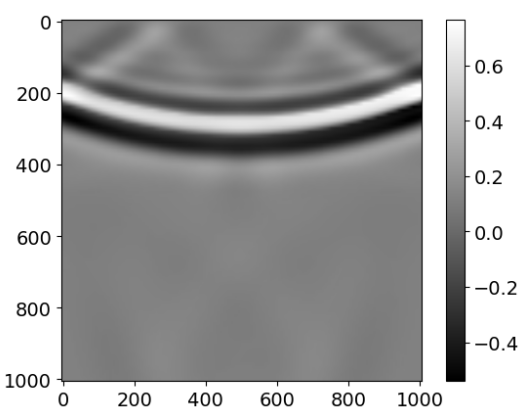

(c) $t=1000$

Figura 3.72: $H A B C$ modificada com a condição A1, com $M=2$ e $\alpha=1.5+0.07(L-M)$, aplicada ao terceiro exemplo bidimensional. 


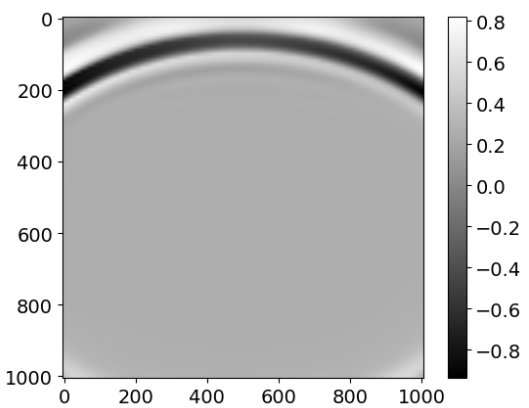

(a) $t=750$

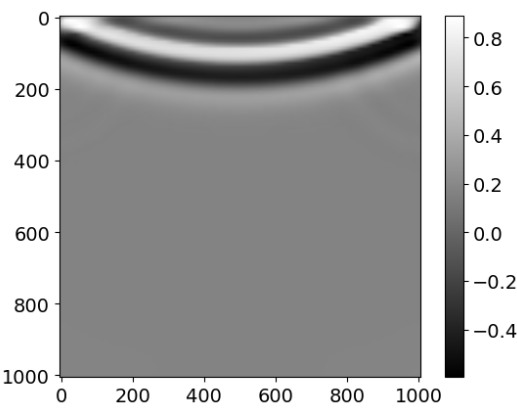

(b) $t=875$

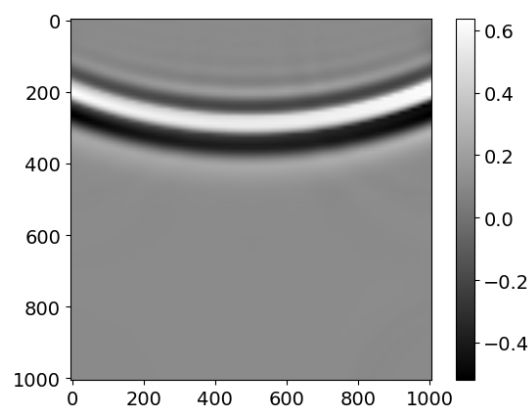

(c) $t=1000$

Figura 3.73: $H A B C$ modificada com a condição A2, com $M=2$ e $\alpha=1.5+0.07(L-M)$, aplicada ao terceiro exemplo bidimensional.

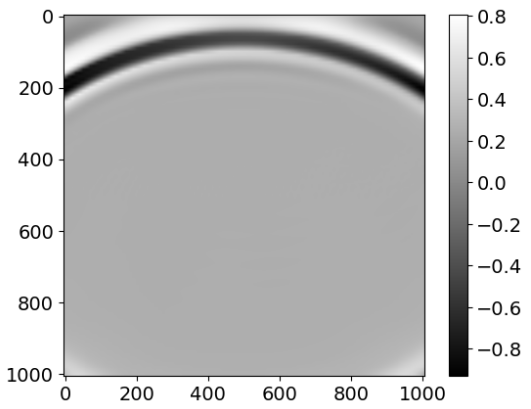

(a) $t=750$

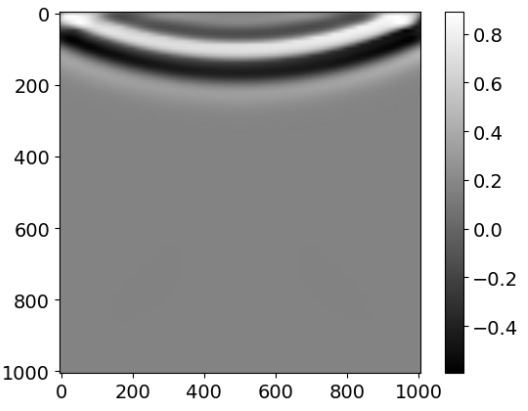

(b) $t=875$

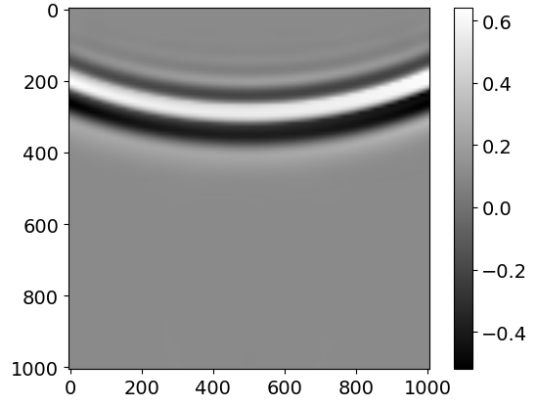

(c) $t=1000$

Figura 3.74: $H A B C$ modificada com a condição de Higdon de segunda ordem, com $M=2, \alpha=1.5+$ $0.07(L-M), \alpha_{1}=0, \alpha_{2}=\frac{\pi}{4}$ e $a_{1}=a_{2}=b_{1}=b_{2}=0.5$, aplicada ao terceiro exemplo bidimensional.

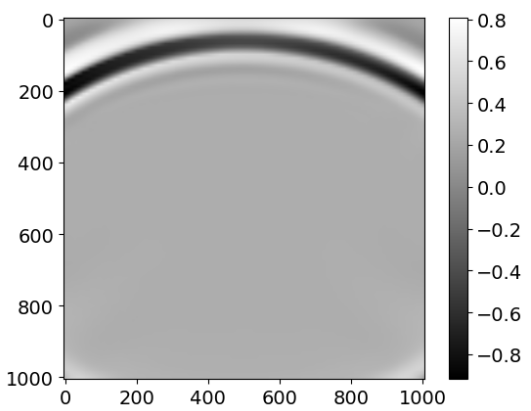

(a) $t=750$

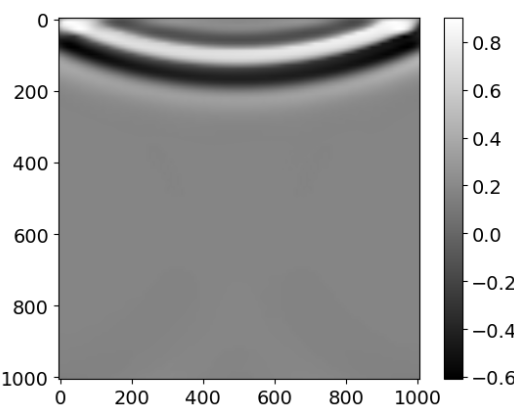

(b) $t=875$

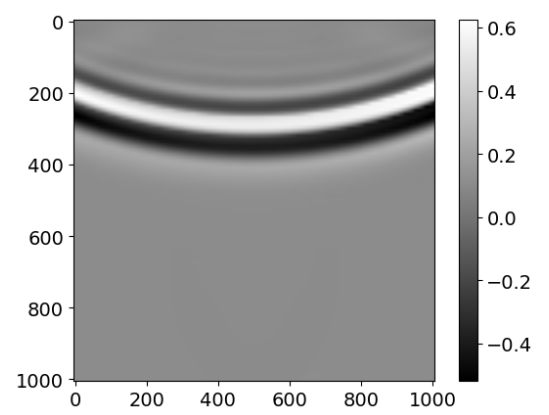

(c) $t=1000$

Figura 3.75: Condição PML para o sistema de primeira ordem, com $R=10^{-4}$, aplicada ao terceiro exemplo bidimensional. 


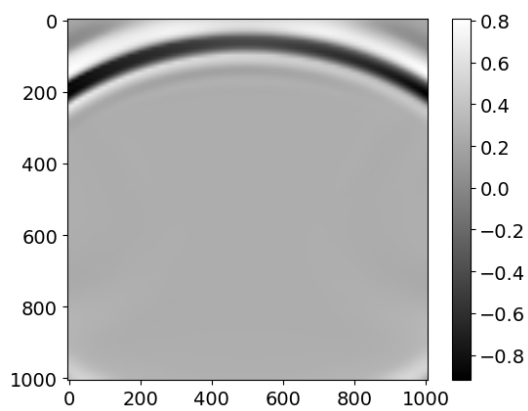

(a) $t=750$

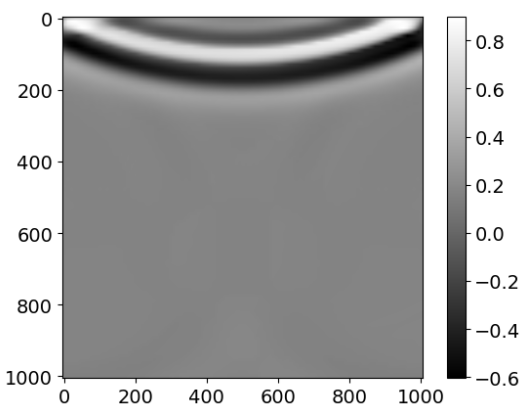

(b) $t=875$

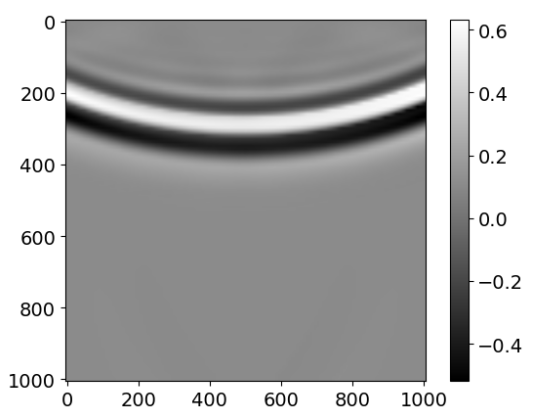

(c) $t=1000$

Figura 3.76: Condição PML para a equação de segunda ordem, com $R=10^{-7}$, aplicada ao terceiro exemplo bidimensional.

\begin{tabular}{c|ccc|ccc} 
& \multicolumn{3}{|c}{ Erro relativo na norma infinito } & \multicolumn{3}{c}{ Erro absoluto na norma 2 } \\
Método & $t=700$ & $t=875$ & $t=1000$ & $t=700$ & $t=875$ & $t=1000$ \\
\hline A2 & 0.1583 & 0.2454 & 0.2392 & 0.0221 & 0.0204 & 0.0242 \\
HABC com A1 & 0.1509 & 0.1579 & 0.2053 & 0.0227 & 0.0215 & 0.0256 \\
HABC com A2 & 0.0169 & 0.0124 & 0.0548 & 0.0012 & 0.0017 & 0.0028 \\
HABC com Higdon 2 & 0.0167 & 0.0042 & 0.0216 & 0.0017 & 0.0013 & 0.0014 \\
Higdon 2 & 0.0653 & 0.1102 & 0.1561 & 0.0098 & 0.0099 & 0.0127 \\
PML 1 & 0.0426 & 0.0282 & 0.0345 & 0.0062 & 0.0038 & 0.0033 \\
PML 2 & 0.0529 & 0.0280 & 0.0369 & 0.0075 & 0.0045 & 0.0037
\end{tabular}

Neste exemplo, é importante observar que a HABC modificada com a condição de Higdon de segunda ordem apresentou os melhores resultados, seguida pela versão com a condição A2 e pelas PMLs. Isso indica que a aplicação da HABC com condições de Higdon pode ser favorável a casos diversos, caracterizando-se como uma possível alternativa à aplicação da PML. As condições de Higdon de ordens mais altas são, portanto, métodos cujo estudo constitui uma possibilidade promissora de trabalhos futuros. 


\section{Capítulo 4}

\section{Conclusões}

No decorrer deste trabalho, abordamos diversas condições de contorno desenvolvidas com o objetivo de viabilizar a simulação numérica do fenômeno de propagação de ondas em domínios ilimitados. Os métodos utilizados foram as condições de absorção A1, A2 e as condições de Higdon de primeira e segunda ordem, além das camadas de amortecimento de Cerjan, Sochacki, as Perfectly Matched Layers propostas para a equação da onda acústica escrita como sistema de primeira ordem e na forma de segunda ordem, a Hybrid Absorbing Boundary Condition original e a versão modificada, além da condição de Dirichlet.

Como vimos no capítulo 2, o uso da condição de Dirichlet como condição de contorno para a equação da onda acústica gera um problema bem-posto, mas não permite a simulação do fenômeno desejado por fornecer reflexão completa das ondas incidentes nos contornos artificiais. Os testes realizados reafirmam os resultados verificados teoricamente.

A condição de Cerjan, que é uma camada de amortecimento, é desenvolvida com o objetivo de aplicar diretamente à equação discretizada um efeito de amortecimento ao longo da camada de absorção. No entanto, essa condição forneceu resultados inferiores aos demais métodos para os testes apresentados. Por ser uma condição de fácil implementação, é possível considerá-la como forma de obter alguma atenuação das ondas incidentes no contorno, embora sua aplicação demande boas escolhas para $\tilde{\lambda}$ e para a espessura da camada acrescentada em torno do domínio de interesse.

A condição de Sochacki, assim como a condição de Cerjan, é uma camada de amortecimento, obtida por meio de modificações realizadas diretamente na equação da onda acústica. Seu uso envolve funções de amortecimento e, neste trabalho, abordamos cinco funções deste tipo e apresentamos a aplicação de três delas a exemplos práticos. Os testes realizados com a condição de Sochacki apresentaram resultados inferiores a grande parte dos obtidos com as demais condições exploradas no presente trabalho, embora a reformulação da equação a ser resolvida possa ser considerada como forma de obter condições de contorno mais eficazes. A escolha do amortecedor não afetou os resultados de forma expressiva, mas a escolha dos parâmetros envolvidos pode alterar o desempenho e a capacidade de amortecimento das camadas acrescentadas em torno do domínio computacional.

As condições de absorção A1 e A2 são desenvolvidas com base em aproximações paraxiais da equação da onda acústica, com o objetivo de anular a propagação de ondas no sentido contrário ao esperado, e sua aplicação é feita diretamente nos contornos artificiais. A condição desenvolvida para a equação unidimensional resulta em absorção completa das ondas incidentes no caso contínuo, mas o caso bidimensional envolve aproximações que afetam significativamente a eficácia dos métodos resultantes. O uso da condição A1 apresentou resultados satisfatórios, especialmente com a velocidade de propagação numérica igual à da onda original, ou seja, com $\lambda=1$. Para o caso bidimensional, tanto a condição A1 quanto a A2 forneceram resultados satisfatórios, tendo a condição A2 apresentado eficácia superior. Apesar disso, é importante salientar que a implementação da condição A2 demanda considerações especiais em relação aos cantos do modelo e faz uso de derivadas de segunda ordem no tempo, envolvendo a solução calculada em três níveis temporais.

As condições de Higdon são condições de absorção mais gerais, cujo objetivo é possibilitar a atenuação de ondas incidentes a ângulos diversos no contorno. A forma geral das condições de Higdon 
permite a atenuação de ondas incidentes a um número arbitrário de ângulos, mas envolve composição de operadores e resulta em derivadas temporais de ordens altas, o que pode inviabilizar sua aplicação sem o uso de funções auxiliares, de modo as condições de primeira e segunda ordens são utilizadas com maior frequência. A aplicação da condição de Higdon de primeira ordem à equação da onda unidimensional teve resultados inferiores aos obtidos por meio da condição A1, embora ambas sejam equivalentes mediante certas condições, e a condição de segunda ordem também apresentou menor atenuação das ondas incidentes. A aplicação ao caso bidimensional, por sua vez, gerou resultados superiores a algumas condições com camadas de amortecimento, especialmente para a condição de segunda ordem, gerando ganho em custo computacional em relação a estas. Embora os resultados obtidos sejam inferiores aos fornecidos por condições como as PMLs e as HABCs, é importante considerar que, aqui, utilizamos $p=1,2$, de modo que é válida a investigação acerca da eficácia dos operadores de ordem mais alta, embora a utilização de funções auxiliares possa aumentar o custo computacional do método.

A PML é um método conhecido por sua eficácia na simulação da propagação de ondas em domínios ilimitados, de modo que diversas formulações desta condição foram elaboradas no decorrer do tempo. No presente trabalho, exploramos duas propostas de PML para a equação da onda acústica. A primeira delas, desenvolvida para o sistema de primeira ordem, gerou os resultados mais satisfatórios para as equações unidimensionais, sendo o melhor método para os dois exemplos apresentados. Sua eficácia também é verificada nos testes bidimensionais, especialmente para o segundo teste. A versão da PML para a equação de segunda ordem apresentou, assim como o método para o sistema, resultados favoráveis à simulação do fenômeno abordado, tanto para o caso unidimensional quanto para o bidimensional, fornecendo a melhor absorção das ondas incidentes para o primeiro teste bidimensional. Ambas as PMLs demandam extensão do domínio e esse fator, associado à inserção de funções auxiliares, pode aumentar consideravelmente seu custo computacional.

As HABCs são condições de contorno que visam atenuar os efeitos da mudança brusca de tratamento da solução entre os pontos do interior do domínio e dos contornos artificiais. Trata-se de uma camada de amortecimento na qual são realizadas combinações entre a solução dada pela equação da onda acústica e a fornecida por uma condição de absorção. Aqui, apresentamos duas formas para a HABC, que sugerem dois modos distintos de realizar a combinação entre as soluções calculadas. Os resultados obtidos a partir das HABCs foram superiores aos fornecidos por quase todas as demais condições nos testes unidimensionais, se assemelhando aos decorrentes do uso das PMLs. Para as equações bidimensionais, o desempenho das HABCs se deu de forma correspondente às condições de absorção associadas, de modo que o uso da A2 proporcionou resultados mais satisfatórios do que os obtidos por meio da aplicação da A1 e da condição de Higdon de segunda ordem. Por se tratar de uma camada de amortecimento, seu custo computacional é maior do que o das condições A1 e A2 e da condição de Higdon, o que deve ser levado em consideração na escolha de um desses métodos.

Os testes realizados, em conjunto com a abordagem teórica, fornecem um panorama geral da eficácia das condições de contorno exploradas na simulação da propagação de ondas em domínio ilimitado. Esse problema pode ser tratado de formas diversas, dada a infinidade de possibilidades relacionadas às condições aqui referidas e à existência de muitas outras ferramentas às quais não nos dedicamos nesta pesquisa. Embora o presente trabalho não se destine a versar sobre todos os aspectos do tema a que se refere, esperamos que os resultados apresentados possam fornecer parâmetros para a escolha de condições de contorno e motivar a realização de pesquisas futuras voltadas, por exemplo, para a implementação de condições de Higdon de ordem mais alta e de outras formulações para a PML, bem como a implementação das condições para a equação da onda elástica em meios isotrópicos conforme os resultados apresentados no apêndice a seguir, e a extensão de tais métodos para casos mais gerais. 


\section{Apêndice A}

\section{Condições de contorno para a equação da onda elástica}

\section{A.1 Ondas sísmicas}

De acordo com Bath [Bat13], caracterizam-se como ondas sísmicas quaisquer movimentos observados por um sismograma, exceto por perturbações dos instrumentos. As ondas sísmicas (ou elásticas) se propagam no interior da Terra ou ao longo de suas camadas de superfície, e são registradas por estações sismográficas em todo o mundo.

As ondas sísmicas são classificadas em dois tipos principais:

1. Ondas de corpo, que se propagam no interior da Terra e se dividem em ondas longitudinais, também denominadas ondas $\mathrm{P}$, e ondas transversais ou de cisalhamento, conhecidas como ondas S;

2. Ondas de superfície que, como o próprio nome diz, se propagam ao longo de uma superfície e são divididas em:

(a) Ondas de Love (ondas L) ou de Rayleigh (R), que seguem a superfície da Terra;

(b) Ondas de Stoneley, que se relacionam às ondas $\mathrm{R}$, mas seguem uma superfície de descontinuidade no interior da Terra;

(c) Ondas de canal, que se propagam em uma camada de velocidade inferior.

Uma partícula atingida por uma onda $\mathrm{P}$ se move para cima e para baixo, na mesma direção de propagação da onda (o efeito, nesse caso, é de compressão e dilatação). Já as ondas S provocam movimento em um plano perpendicular à direção de propagação da onda, movimento este que é dividido em uma componente horizontal (SH) e uma vertical (SV).

No caso das ondas de superfície, a onda L gera um movimento que coincide com SH, ou seja, é um movimento transversal horizontal. A onda $R$, por sua vez, gera um movimento elíptico e o plano da elipse é vertical, contido no plano de propagação.

Na descrição matemática da propagação das ondas, é comum admitir condições simplificadoras, tais como:

1. O deslocamento relativo entre duas partículas é infinitesimalmente pequeno;

2. O estresse é uma função homogênea linear da tensão e vice-versa (nesse caso, o material é dito perfeitamente elástico);

3. O material é isotrópico, isto é, os parâmetros elásticos independem da direção (caso contrário, o material é dito anisotrópico ou aleotrópico);

4. Forças externas podem ser desprezadas. 
Há casos em que é necessário eliminar algumas das hipóteses acima, mas isso não é comum. Sendo satisfeitas essas condições, as propriedades elásticas dos materiais podem ser caracterizadas por dois parâmetros:

$k=$ módulo de incompressibilidade ou módulo volumétrico,

$\mu=$ módulo de rigidez ou de cisalhamento.

O parâmetro $k$ é frequentemente relacionado a outro parâmetro, $\lambda$, da seguinte forma:

$$
k=\lambda+\frac{2}{3} \mu,
$$

e os parâmetros $\lambda$ e $\mu$ são conhecidos como coeficientes de Lamé. Esses valores são utilizados para descrever as velocidades $v_{p}$ e $v_{s}$ das ondas $\mathrm{P}$ e S, respectivamente. Se $\rho$ é a densidade do material, tais velocidades são as seguintes:

$$
v_{p}=\sqrt{\frac{k+\frac{4}{3} \mu}{\rho}}, v_{s}=\sqrt{\frac{\mu}{\rho}} .
$$

Das relações acima, é possível observar que $v_{p}>v_{s}$, ou seja, as ondas $\mathrm{P}$ sempre se propagam em velocidade maior do que as ondas S. Em sismologia, é comum que os estudos se concentrem nesses dois tipos de onda, e essa é a abordagem adotada para o desenvolvimento das próximas seções.

\section{A.2 Equação da onda sísmica no domínio contínuo}

Em um meio heterogêneo contínuo, as ondas sísmicas correspondem localmente às soluções de determinadas equações diferenciais parciais, bem como equações integrais. Para obter uma equação que represente sua propagação em um meio contínuo, é necessário compreender alguns conceitos intimamente relacionados a estes fenômenos. Apresentamos brevemente estes conceitos a seguir, de acordo com Shearer [She09]. Para tornar essa apresentação mais intuitiva, utilizamos a abordagem tridimensional, possibilitando a aplicação mais ampla dos conceitos físicos intrínsecos ao fenômeno analisado.

Consideremos um plano infinitesimal orientado de forma arbitrária por um vetor unitário $\hat{\boldsymbol{n}}$ em um meio elástico, homogêneo e em equilíbrio estático. O tensor de estresse em um sistema tridimensional de coordenadas cartesianas é dado por

$$
\boldsymbol{\sigma}=\left(\begin{array}{lll}
\sigma_{11} & \sigma_{12} & \sigma_{13} \\
\sigma_{21} & \sigma_{22} & \sigma_{23} \\
\sigma_{31} & \sigma_{32} & \sigma_{33}
\end{array}\right)=\left(\begin{array}{ccc}
t_{1}(\hat{x}) & t_{1}(\hat{y}) & t_{1}(\hat{z}) \\
t_{2}(\hat{x}) & t_{2}(\hat{y}) & t_{2}(\hat{z}) \\
t_{3}(\hat{x}) & t_{3}(\hat{y}) & t_{3}(\hat{z})
\end{array}\right),
$$

onde $t_{i}(\hat{j})$ é a i-ésima componente da força atuando na dimensão $j$ do meio. Devido ao equilíbrio estático, podemos afirmar que a matriz $\sigma$ é simétrica, ou seja

$$
\boldsymbol{\sigma}=\left(\begin{array}{lll}
\sigma_{11} & \sigma_{12} & \sigma_{13} \\
\sigma_{12} & \sigma_{22} & \sigma_{23} \\
\sigma_{13} & \sigma_{23} & \sigma_{33}
\end{array}\right)
$$

O tensor de estresse é o operador linear a partir do qual podemos obter o vetor de tração $\mathbf{t}(\hat{\boldsymbol{n}})$ através de um plano orientado pelo vetor normal $\hat{\boldsymbol{n}}$. Isso porque

$$
\mathbf{t}(\hat{\boldsymbol{n}})=\left(\begin{array}{c}
t_{x}(\hat{\boldsymbol{n}}) \\
t_{y}(\hat{\boldsymbol{n}}) \\
t_{z}(\hat{\boldsymbol{n}})
\end{array}\right)=\left(\begin{array}{ccc}
\sigma_{11} & \sigma_{12} & \sigma_{13} \\
\sigma_{12} & \sigma_{22} & \sigma_{23} \\
\sigma_{13} & \sigma_{23} & \sigma_{33}
\end{array}\right)\left(\begin{array}{c}
\hat{n}_{1} \\
\hat{n}_{2} \\
\hat{n}_{3}
\end{array}\right) .
$$

A localização de uma partícula no tempo $t$ relativamente à sua posição em um tempo de referência $t_{0}$ pode ser representada por um campo de vetores, ao qual nos referimos como campo de deslocamento e que pode ser escrito como 


$$
\mathbf{u}\left(\mathbf{r}_{0}, t\right)=\mathbf{r}-\mathbf{r}_{0},
$$

sendo $\mathbf{r}$ e $\mathbf{r}_{0}$ as localizações da partícula nos tempos $t$ e $t_{0}$, respectivamente. Essa descrição do movimento é conhecida como descrição Lagrangiana e, expandindo $\mathbf{u}$ em série de Taylor e usando a notação $\mathbf{d}=\left(d_{1}, d_{2}, d_{3}\right)=\mathbf{x}-\mathbf{x}_{0}$, obtemos

$$
\mathbf{u}(\mathbf{x})=\mathbf{u}\left(\mathbf{x}_{0}\right)+\left(\begin{array}{ccc}
\frac{\partial u_{1}}{\partial x} & \frac{\partial u_{1}}{\partial y} & \frac{\partial u_{1}}{\partial z} \\
\frac{\partial u_{2}}{\partial x} & \frac{\partial u_{2}}{\partial y} & \frac{\partial u_{2}}{\partial z} \\
\frac{\partial u_{3}}{\partial x} & \frac{\partial u_{3}}{\partial y} & \frac{\partial u_{3}}{\partial z}
\end{array}\right)\left(\begin{array}{c}
d_{1} \\
d_{2} \\
d_{3}
\end{array}\right)=\mathbf{J d} .
$$

A matriz $\mathbf{J}$ pode ser escrita como a soma de duas matrizes, uma simétrica e uma antissimétrica, da forma

$$
\begin{gathered}
\mathbf{e}=\left(\begin{array}{ccc}
\frac{\partial u_{1}}{\partial x} & \frac{1}{2}\left(\frac{\partial u_{1}}{\partial y}+\frac{\partial u_{2}}{\partial x}\right) & \frac{1}{2}\left(\frac{\partial u_{1}}{\partial z}+\frac{\partial u_{3}}{\partial x}\right) \\
\frac{1}{2}\left(\frac{\partial u_{2}}{\partial x}+\frac{\partial u_{1}}{\partial y}\right) & \frac{\partial u_{2}}{\partial y} & \frac{1}{2}\left(\frac{\partial u_{2}}{\partial z}+\frac{\partial u_{3}}{\partial y}\right) \\
\frac{1}{2}\left(\frac{\partial u_{3}}{\partial x}+\frac{\partial u_{1}}{\partial z}\right) & \frac{1}{2}\left(\frac{\partial u_{3}}{\partial y}+\frac{\partial u_{2}}{\partial z}\right) & \frac{\partial u_{3}}{\partial z}
\end{array}\right) \\
\Omega=\left(\begin{array}{ccc}
0 & \frac{1}{2}\left(\frac{\partial u_{1}}{\partial y}-\frac{\partial u_{2}}{\partial x}\right) & \frac{1}{2}\left(\frac{\partial u_{1}}{\partial z}-\frac{\partial u_{3}}{\partial x}\right) \\
-\frac{1}{2}\left(\frac{\partial u_{2}}{\partial x}-\frac{\partial u_{1}}{\partial y}\right) & 0 & \frac{1}{2}\left(\frac{\partial u_{2}}{\partial z}-\frac{\partial u_{3}}{\partial y}\right) \\
-\frac{1}{2}\left(\frac{\partial u_{3}}{\partial x}-\frac{\partial u_{1}}{\partial z}\right) & -\frac{1}{2}\left(\frac{\partial u_{3}}{\partial y}-\frac{\partial u_{2}}{\partial z}\right) & 0
\end{array}\right),
\end{gathered}
$$

onde o tensor e é o tensor de tensão, e $\Omega$ é o tensor de rotação.

Se a aplicação de $\mathbf{J}$ a um material causar mudança em seu volume, o aumento relativo de volume $\Delta V=\frac{\left(V-V_{0}\right)}{V_{0}}$ é dado por $\nabla \cdot u=\operatorname{tr}(\mathbf{e})$.

Os tensores de tensão e estresse estão linearmente relacionados a partir da seguinte equação:

$$
\sigma_{i j}=c_{i j k l} e_{k l}=\sum_{k=1}^{3} \sum_{l=1}^{3} c_{i j k l} e_{k l} .
$$

A equação (A.1) é frequentemente chamada de lei generalizada de Hooke e aqui assumimos elasticidade perfeita do material, de modo que não há perda de energia com sua deformação. O tensor elástico $c_{i j k l}$ é um tensor de quarta ordem com $81\left(3^{4}\right)$ componentes, das quais somente 21 devem ser independentes, devido a considerações termodinâmicas e simetrias. Para os meios isotrópicos, as componentes independentes podem ser representadas pelos coeficientes de Lamé $\lambda$ e $\mu$, com os quais cada $c_{i j k l}$ se relaciona por meio da equação

$$
c_{i j k l}=\lambda \delta_{i j} \delta_{k l}+\mu\left(\delta_{i k} \delta_{j l}+\delta_{i l} \delta_{j k}\right),
$$

$\operatorname{com} \delta_{m n}$ sendo o delta de Kronecker. Assim, temos a relação

$$
\boldsymbol{\sigma}_{i j}=\lambda \delta_{i j} \operatorname{tr}(\mathbf{e})+2 \mu \mathbf{e}_{i j} .
$$

Consideremos agora as forças que atuam sobre um cubo infinitesimal em um sistema de coordenadas $\left(x_{1}, x_{2}, x_{3}\right)$. As forças atuando em cada face do cubo são obtidas a partir do produto entre o vetor de tração e a área da face em questão. Por exemplo, a força em uma face normal a $\mathbf{x}_{1}$ é dada por

$$
F\left(\mathbf{x}_{1}\right)=\mathbf{t}\left(\mathbf{x}_{1}\right) d x_{2} d x_{3}=\boldsymbol{\sigma} \mathbf{x}_{1} d x_{2} d x_{3}=\left(\begin{array}{c}
\sigma_{11} \\
\sigma_{21} \\
\sigma_{31}
\end{array}\right) d x_{2} d x_{3} .
$$

As forças resultantes atuando no cubo só serão não-nulas se gradientes espaciais estiverem 
presentes no campo de estresse. Nesse caso, a força resultante no plano normal a $\mathbf{x}_{1}$ é

$$
\mathbf{F}\left(\mathbf{x}_{1}\right)=\frac{\partial}{\partial x_{1}}\left(\begin{array}{l}
\sigma_{11} \\
\sigma_{21} \\
\sigma_{31}
\end{array}\right) d x_{1} d x_{2} d x_{3} .
$$

Desse modo, cada componente $\mathbf{F}_{i}$ de $\mathbf{F}$ será dada por

$$
\mathbf{F}_{i}=\sum_{j=1}^{3} \frac{\partial \sigma_{i j}}{\partial x_{j}} d x_{1} d x_{2} d x_{3}
$$

Assumimos a possibilidade de existir uma força de corpo $\mathbf{F}^{C}$ atuando no cubo de forma proporcional ao seu volume, ou seja:

$$
\mathbf{F}_{i}^{C}=f_{i} d x_{1} d x_{2} d x_{3}
$$

A massa $m$ do cubo é dada por

$$
m=\rho d x_{1} d x_{2} d x_{3},
$$

onde $\rho$ é a densidade do material. A aceleração do cubo é obtida a partir da segunda derivada do deslocamento em relação ao tempo. Usando as equações (A.3), (A.4) e (A.5) em $\mathbf{F}=m \cdot a$, temos

$$
\begin{gathered}
\rho d x_{1} d x_{2} d x_{3} \cdot \frac{\partial^{2} u_{i}}{\partial t^{2}}=f_{i} d x_{1} d x_{2} d x_{3}+d x_{1} d x_{2} d x_{3} \sum_{j=1}^{3} \frac{\partial \sigma_{i j}}{\partial x_{j}} \\
\Rightarrow \rho \frac{\partial^{2} u_{i}}{\partial t^{2}}=\sum_{j=1}^{3} \frac{\partial \sigma_{i j}}{\partial x_{j}}+f_{i} .
\end{gathered}
$$

Lembrando da relação entre estresse e tensão (A.2) e de que $e_{i j}=\frac{1}{2}\left(\frac{\partial u_{i}}{\partial x_{j}}+\frac{\partial u_{j}}{\partial x_{i}}\right)$, substituímos essas relações em (A.6) e obtemos

$$
\begin{gathered}
\rho \frac{\partial^{2} u_{i}}{\partial t^{2}}=\sum_{k=1}^{3}\left\{\sum_{j=1}^{3} \frac{\partial}{\partial x_{j}}\left[\lambda \delta_{i j} \frac{\partial u_{k}}{\partial x_{k}}+\mu\left(\frac{\partial u_{j}}{\partial x_{i}}+\frac{\partial u_{i}}{\partial x_{j}}\right)\right]\right\}+f_{i} \\
\rho \frac{\partial^{2} u_{i}}{\partial t^{2}}=\sum_{k=1}^{3}\left(\frac{\partial}{\partial x_{i}} \lambda \frac{\partial u_{k}}{\partial x_{k}}+\lambda \frac{\partial^{2} u_{k}}{\partial x_{i} \partial x_{k}}\right)+\sum_{j=1}^{3}\left[\frac{\partial}{\partial x_{j}} \mu\left(\frac{\partial u_{j}}{\partial x_{i}}+\frac{\partial u_{i}}{\partial x_{j}}\right)\right]+\sum_{j=1}^{3}\left[\mu \frac{\partial}{\partial x_{j}}\left(\frac{\partial u_{j}}{\partial x_{i}}+\frac{\partial u_{i}}{\partial x_{j}}\right)\right]+f_{i} \\
\rho \frac{\partial^{2} u_{i}}{\partial t^{2}}=\sum_{k=1}^{3}\left(\frac{\partial}{\partial x_{i}} \lambda \frac{\partial u_{k}}{\partial x_{k}}\right)+\sum_{j=1}^{3}\left[\frac{\partial}{\partial x_{j}} \mu\left(\frac{\partial u_{j}}{\partial x_{i}}+\frac{\partial u_{i}}{\partial x_{j}}\right)\right]+\sum_{k=1}^{3}\left(\lambda \frac{\partial^{2} u_{k}}{\partial x_{i} \partial x_{k}}\right) \\
+\sum_{j=1}^{3}\left(\mu \frac{\partial^{2} u_{j}}{\partial x_{j} \partial x_{i}}\right)+\sum_{j=1}^{3}\left(\mu \frac{\partial^{2} u_{i}}{\partial x_{j}^{2}}\right)+f_{i} .
\end{gathered}
$$

Se $\ddot{\mathbf{u}}=\frac{\partial \mathbf{u}}{\partial t^{2}}$ e $\mathbf{f}=\left(f_{1}, f_{2}, f_{3}\right)$, temos

$$
\rho \ddot{\mathbf{u}}=\nabla \lambda \cdot(\nabla u)+\nabla \mu \cdot\left[\nabla u+(\nabla u)^{T}\right]+(\lambda+\mu) \nabla \nabla u+\mu \nabla^{2} u+\mathbf{f} .
$$

Podemos usar a identidade 


$$
\nabla \times \nabla \times u=\nabla \nabla u-\nabla^{2} u
$$

e assim, obtermos a equação

$$
\rho \ddot{\mathbf{u}}=\nabla \lambda \cdot(\nabla u)+\nabla \mu \cdot\left[\nabla u+(\nabla u)^{T}\right]+(\lambda+2 \mu) \nabla \nabla u-\mu \nabla \times \nabla \times u+\mathbf{f} .
$$

A equação acima depende dos gradientes de $\lambda$ e $\mu$ e essa questão pode ser abordada de duas formas. A primeira é considerando que, se a velocidade varia em função da profundidade, então em cada camada os parâmetros $\lambda$ e $\mu$ não variam e os gradientes serão nulos. A segunda possibilidade é observar que os gradientes em questão variam com $\frac{1}{\omega} \mathrm{e}$, em altas frequências, tais termos tendem a 0. Nesses casos, se pudermos assumir os gradientes como nulos, a equação resultante é

$$
\rho \ddot{\mathbf{u}}=(\lambda+2 \mu) \nabla \nabla u-\mu \nabla \times \nabla \times u+\mathbf{f} .
$$

Desenvolvendo a equação acima, podemos verificar que, em três dimensões (denotadas por $x, y$ e $z$ ), a mesma equivale a

$$
\begin{aligned}
\frac{\partial^{2} u_{1}}{\partial t^{2}} & =\frac{1}{\rho}\left[(\lambda+2 \mu) \frac{\partial^{2} u_{1}}{\partial x^{2}}+(\lambda+\mu)\left(\frac{\partial^{2} u_{2}}{\partial x \partial y}+\frac{\partial^{2} u_{3}}{\partial x \partial z}\right)+\mu\left(\frac{\partial^{2} u_{1}}{\partial y^{2}}+\frac{\partial^{2} u_{1}}{\partial z^{2}}\right)+f_{x}\right] \\
\frac{\partial^{2} u_{2}}{\partial t^{2}} & =\frac{1}{\rho}\left[(\lambda+2 \mu) \frac{\partial^{2} u_{2}}{\partial y^{2}}+(\lambda+\mu)\left(\frac{\partial^{2} u_{1}}{\partial x \partial y}+\frac{\partial^{2} u_{3}}{\partial y \partial z}\right)+\mu\left(\frac{\partial^{2} u_{2}}{\partial x^{2}}+\frac{\partial^{2} u_{2}}{\partial z^{2}}\right)+f_{y}\right] \\
\frac{\partial^{2} u_{3}}{\partial t^{2}} & =\frac{1}{\rho}\left[(\lambda+2 \mu) \frac{\partial^{2} u_{3}}{\partial z^{2}}+(\lambda+\mu)\left(\frac{\partial^{2} u_{1}}{\partial x \partial z}+\frac{\partial^{2} u_{2}}{\partial y \partial z}\right)+\mu\left(\frac{\partial^{2} u_{3}}{\partial x^{2}}+\frac{\partial^{2} u_{3}}{\partial y^{2}}\right)+f_{z}\right] .
\end{aligned}
$$

Para duas dimensões, as equações serão

$$
\begin{aligned}
\frac{\partial^{2} u_{1}}{\partial t^{2}} & =\frac{1}{\rho}\left[(\lambda+2 \mu) \frac{\partial^{2} u_{1}}{\partial x^{2}}+(\lambda+\mu) \frac{\partial^{2} u_{2}}{\partial x \partial y}+\mu \frac{\partial^{2} u_{1}}{\partial y^{2}}+f_{x}\right] \\
\frac{\partial^{2} u_{2}}{\partial t^{2}} & =\frac{1}{\rho}\left[(\lambda+2 \mu) \frac{\partial^{2} u_{2}}{\partial y^{2}}+(\lambda+\mu) \frac{\partial^{2} u_{2}}{\partial x \partial y}+\mu \frac{\partial^{2} u_{2}}{\partial x^{2}}+f_{y}\right] .
\end{aligned}
$$

Aqui, $\mathbf{u}=\left(u_{1}, u_{2}\right)$, ou seja, $u_{1}$ e $u_{2}$ são as componentes do vetor de deslocamento. A equação (A.8) é conhecida como formulação de deslocamento da equação da onda elástica. Por simplicidade, em alguns casos, utilizaremos a seguinte representação da equação acima:

$$
\begin{aligned}
& \frac{\partial^{2} u_{1}}{\partial t^{2}}=\alpha^{2} \frac{\partial^{2} u_{1}}{\partial x^{2}}+\beta^{2} \frac{\partial^{2} u_{1}}{\partial y^{2}}+\left(\alpha^{2}-\beta^{2}\right) \frac{\partial^{2} u_{2}}{\partial x \partial y}+f_{1}(x, y, t) \\
& \frac{\partial^{2} u_{2}}{\partial t^{2}}=\beta^{2} \frac{\partial^{2} u_{2}}{\partial x^{2}}+\alpha^{2} \frac{\partial^{2} u_{2}}{\partial y^{2}}+\left(\alpha^{2}-\beta^{2}\right) \frac{\partial^{2} u_{1}}{\partial x \partial y}+f_{2}(x, y, t) .
\end{aligned}
$$

Podemos ainda considerar a relação entre $\boldsymbol{\sigma}$ e $\mathbf{e}$

$$
\boldsymbol{\sigma}(x, t)=\int_{-\infty}^{\infty} \dot{\mathbf{C}}\left(\mathbf{x}, t-t^{\prime}\right): \mathbf{e}\left(\mathbf{x}, t^{\prime}\right) d t^{\prime}
$$

onde $\mathbf{C}$ é o tensor elástico definido por $c_{i j k l}$ e $(\mathbf{C}: \mathbf{e})_{k l}=\sum_{i, j=1}^{n} e_{i j} c_{i j k l}$.

As relações de simetria de $\mathbf{C}(x, t)$ nos permitem escrever

$$
\boldsymbol{\sigma}(x, t)=\int_{-\infty}^{\infty} \dot{\mathbf{C}}\left(\mathbf{x}, t-t^{\prime}\right): \nabla \mathbf{u}\left(\mathbf{x}, t^{\prime}\right) d t^{\prime}
$$


A dependência do tempo em $\mathbf{C}(\mathbf{x}, t)$ está relacionada à transformação de energia em calor e, no caso de meios não-dissipativos, temos

$$
\mathbf{C}(\mathbf{x}, t)=\mathbf{C}(\mathbf{x}) H(t)
$$

onde $H(t)$ é a função de Heaviside. Isso nos permite escrever a equação (A.11) da seguinte forma:

$$
\boldsymbol{\sigma}(\mathbf{x}, t)=\mathbf{C}(\mathbf{x}): \nabla \mathbf{u}(\mathbf{x}, t) .
$$

Por fim, derivando a equação (A.12) em relação a $t$ e substituindo $\dot{\mathbf{u}}$ por $\mathbf{v}$, obtemos a formulação velocidade-estresse da equação da onda elástica

$$
\begin{aligned}
& \rho(\mathbf{x}) \dot{\mathbf{v}}-\nabla \cdot \boldsymbol{\sigma}(\mathbf{x}, t)=f(\mathbf{x}, t) \\
& \dot{\boldsymbol{\sigma}}(\mathbf{x}, t)-\mathbf{C}(\mathbf{x}): \nabla \mathbf{v}(\mathbf{x}, t)=0 .
\end{aligned}
$$

Como $c_{i j k l}=\lambda \delta_{i j} \delta_{k l}+\mu\left(\delta_{i k} \delta_{j l}+\delta_{i l} \delta_{j k}\right)$, qualquer dificuldade relacionada a $\mathbf{C}$ é eliminada. Uma abordagem geral acerca da equação da onda elástica pode ser encontrada em [VECA $\left.{ }^{+} 12\right]$ e [Cha04].

\section{A.3 Condições de contorno para a equação da onda elástica}

A seguir, apresentamos as formulações das condições de contorno abordadas no capítulo 2 para a equação da onda elástica.

No caso da equação da onda elástica, na qual são utilizadas derivadas em mais de uma dimensão espacial simultaneamente, as discretizações de uma mesma função podem ser feitas para cada dimensão separadamente. Utilizando aproximações de segunda ordem para as derivadas, temos que a equação da onda elástica na formulação de deslocamento em meios isotrópicos, em sua versão discreta, será

$$
\begin{gathered}
\left(u_{1}\right)_{i, j}^{n+1}=2\left(u_{1}\right)_{i, j}^{n}-\left(u_{1}\right)_{i, j}^{n-1}+\frac{\Delta t^{2}}{\rho_{i, j}}\left[(\lambda+2 \mu)\left(\frac{\left(u_{1}\right)_{i+1, j}^{n}-2\left(u_{1}\right)_{i, j}^{n}+\left(u_{1}\right)_{i-1, j}^{n}}{\Delta x^{2}}\right)\right. \\
+(\lambda+\mu)\left(\frac{\left(u_{2}\right)_{i+1, j+1}^{n}-\left(u_{2}\right)_{i+1, j-1}^{n}-\left(u_{2}\right)_{i-1, j+1}^{n}+\left(u_{2}\right)_{i-1, j-1}^{n}}{4 \Delta x \Delta y}\right) \\
\left.+\mu\left(\frac{\left(u_{1}\right)_{i, j+1}^{n}-2\left(u_{1}\right)_{i, j}^{n}+\left(u_{1}\right)_{i, j-1}^{n}}{\Delta y^{2}}\right)\right] \\
\left(u_{2}\right)_{i, j}^{n+1}=2\left(u_{2}\right)_{i, j}^{n}-\left(u_{2}\right)_{i, j}^{n-1}+\frac{\Delta t^{2}}{\rho_{i, j}}\left[(\lambda+2 \mu)\left(\frac{\left(u_{2}\right)_{i, j+1}^{n}-2\left(u_{2}\right)_{i, j}^{n}+\left(u_{2}\right)_{i, j-1}^{n}}{\Delta y^{2}}\right)\right. \\
+(\lambda+\mu)\left(\frac{\left(u_{1}\right)_{i+1, j+1}^{n}-\left(u_{1}\right)_{i+1, j-1}^{n}-\left(u_{1}\right)_{i-1, j+1}^{n}+\left(u_{1}\right)_{i-1, j-1}^{n}}{4 \Delta x \Delta y}\right) \\
\left.+\mu\left(\frac{\left(u_{2}\right)_{i+1, j}^{n}-2\left(u_{2}\right)_{i, j}^{n}+\left(u_{2}\right)_{i-1, j}^{n}}{\Delta x^{2}}\right)\right] .
\end{gathered}
$$

Um estudo mais detalhado acerca da equação da onda elástica em suas diversas formulações e seu tratamento numérico por meio de discretizações pode ser encontrado em [ $\left.\mathrm{MKG}^{+} 07\right]$, trabalho no qual também são abordados Métodos de Elementos Finitos e staggered grids.

As condições de Dirichlet e Neumann apresentadas no capítulo 1 podem ser aplicadas separadamente a cada componente da solução da equação da onda elástica, ou seja, em um domínio $[-a, a] \times[-b, b]$, a condição de Dirichlet passa a ser 


$$
\begin{aligned}
& u_{1}( \pm a, y, t)=0, u_{1}(x, \pm b, t)=0 \\
& u_{2}( \pm a, y, t)=0, u_{2}(x, \pm b, t)=0
\end{aligned}
$$

e a condição de Neumann,

$$
\begin{aligned}
& \frac{\partial u_{1}}{\partial x}( \pm a, y, t)=0, \frac{\partial u_{1}}{\partial x}(x, \pm b, t)=0, \\
& \frac{\partial u_{2}}{\partial x}( \pm a, y, t)=0, \frac{\partial u_{2}}{\partial x}(x, \pm b, t)=0 .
\end{aligned}
$$

O mesmo vale para a condição proposta por Cerjan, apresentada na seção 2.3.1, de modo que tal condição pode ser aplicada à equação da onda elástica na formulação de deslocamento da seguinte forma, adotando-se a discretização (A.15)-(A.16):

$$
\begin{gathered}
\left(u_{1}\right)_{i, j}^{n+1}=G\left(x_{i}, y_{j}\right)\left\{2\left(u_{1}\right)_{i, j}^{n}-\left(u_{1}\right)_{i, j}^{n-1}+\frac{\Delta t^{2}}{\rho_{i, j}}\left[(\lambda+2 \mu)\left(\frac{\left(u_{1}\right)_{i+1, j}^{n}-2\left(u_{1}\right)_{i, j}^{n}+\left(u_{1}\right)_{i-1, j}^{n}}{\Delta x^{2}}\right)\right.\right. \\
\left.\left.+(\lambda+\mu)\left(\frac{\left.\left(u_{2}\right)_{i+1, j+1}^{n}-\left(u_{2}\right)_{i+1, j-1}^{n}-\left(u_{2}\right)_{i-1, j+1}^{n}+\left(u_{2}\right)_{i-1, j-1}^{n}\right)}{4 \Delta x \Delta y}\right)\right]\right\} \\
\left.\left.+\mu\left(\frac{\left(u_{1}\right)_{i, j+1}^{n}-2\left(u_{1}\right)_{i, j}^{n}+\left(u_{1}\right)_{i, j-1}^{n}}{\Delta y^{2}}\right)\right]\right\} \\
\left(u_{2}\right)_{i, j}^{n+1}=G\left(x_{i}, y_{j}\right)\left\{2\left(u_{2}\right)_{i, j}^{n}-\left(u_{2}\right)_{i, j}^{n-1}+\frac{\Delta t^{2}}{\rho_{i, j}}\left[(\lambda+2 \mu)\left(\frac{\left(u_{2}\right)_{i, j+1}^{n}-2\left(u_{2}\right)_{i, j}^{n}+\left(u_{2}\right)_{i, j-1}^{n}}{\Delta y^{2}}\right)\right.\right. \\
+(\lambda+\mu)\left(\frac{\left(u_{1}\right)_{i+1, j+1}^{n}-\left(u_{1}\right)_{i+1, j-1}^{n}-\left(u_{1}\right)_{i-1, j+1}^{n}+\left(u_{2}\right)_{i-1, j-1}^{n}}{4 \Delta x \Delta y}\right) \\
\left.\left.+\mu\left(\frac{\left(u_{2}\right)_{i+1, j}^{n}-2\left(u_{2}\right)_{i, j}^{n}+\left(u_{2}\right)_{i-1, j}^{n}}{\Delta x^{2}}\right)\right]\right\} .
\end{gathered}
$$

No caso do método proposto por Sochacki, ao qual se refere a seção 2.3.2, a aplicação à equação da onda elástica é dada pelo seguinte sistema de equações, no qual a função $A(x, y)$ possui as mesmas propriedades admitidas para o método desenvolvido para a equação da onda acústica:

$$
\begin{aligned}
& \frac{\partial^{2} u_{1}}{\partial t^{2}}+2 A(x, y) \frac{\partial u_{1}}{\partial t}=\alpha^{2} \frac{\partial^{2} u_{1}}{\partial x^{2}}+\beta^{2} \frac{\partial^{2} u_{1}}{\partial y^{2}}+\left(\alpha^{2}-\beta^{2}\right) \frac{\partial^{2} u_{2}}{\partial x \partial y}+f_{1}(x, y, t) \\
& \frac{\partial^{2} u_{2}}{\partial t^{2}}+2 A(x, y) \frac{\partial u_{2}}{\partial t}=\beta^{2} \frac{\partial^{2} u_{2}}{\partial x^{2}}+\alpha^{2} \frac{\partial^{2} u_{2}}{\partial y^{2}}+\left(\alpha^{2}-\beta^{2}\right) \frac{\partial^{2} u_{1}}{\partial x \partial y}+f_{2}(x, y, t),
\end{aligned}
$$

com as condições iniciais:

$$
\begin{aligned}
& \left(\begin{array}{l}
u_{1} \\
u_{2}
\end{array}\right)(x, y, 0)=\left(\begin{array}{l}
g_{1} \\
g_{2}
\end{array}\right)(x, y) \\
& \left(\begin{array}{c}
\frac{\partial u_{1}}{\partial t} \\
\frac{\partial u_{2}}{\partial t}
\end{array}\right)(x, y, 0)=\left(\begin{array}{l}
h_{1} \\
h_{2}
\end{array}\right)(x, y) .
\end{aligned}
$$


A discretização da condição de contorno obtida pode ser feita conforme os operadores já abordados para a equação da onda elástica e para a derivada temporal de primeira ordem. Assim, a forma discreta da condição de Sochacki nesse caso é

$$
\begin{aligned}
\left(u_{1}\right)_{i, j}^{n+1}= & \left(\frac{\Delta t^{2}}{1+\Delta t A\left(x_{i}, y_{j}\right)}\right)\left[\frac{2\left(u_{1}\right)_{i, j}^{n}}{\Delta t^{2}}-\left(\frac{1-\Delta t A\left(x_{i}, y_{j}\right)}{\Delta t^{2}}\right)\left(u_{1}\right)_{i, j}^{n-1}\right]+ \\
& \left(\frac{\Delta t^{2}}{1+\Delta t A\left(x_{i}, y_{j}\right)}\right) \frac{1}{\rho_{i, j}}\left[(\lambda+2 \mu)\left(\frac{\left(u_{1}\right)_{i+1, j}^{n}-2\left(u_{1}\right)_{i, j}^{n}+\left(u_{1}\right)_{i-1, j}^{n}}{\Delta x^{2}}\right)\right. \\
+(\lambda+\mu) & \left(\frac{\left(u_{2}\right)_{i+1, j+1}^{n}-\left(u_{2}\right)_{i+1, j-1}^{n}-\left(u_{2}\right)_{i-1, j+1}^{n}+\left(u_{2}\right)_{i-1, j-1}^{n}}{4 \Delta x \Delta y}\right) \\
+\mu & \left.\left(\frac{\left(u_{1}\right)_{i, j+1}^{n}-2\left(u_{1}\right)_{i, j}^{n}+\left(u_{1}\right)_{i, j-1}^{n}}{\Delta y^{2}}\right)\right] \\
\left(u_{2}\right)_{i, j}^{n+1}= & \left(\frac{\Delta t^{2}}{1+\Delta t A\left(x_{i}, y_{j}\right)}\right)\left[\frac{2\left(u_{2}\right)_{i, j}^{n}}{\Delta t^{2}}-\left(\frac{1-\Delta t A\left(x_{i}, y_{j}\right)}{\Delta t^{2}}\right)\left(u_{2}\right)_{i, j}^{n-1}\right]+ \\
& \left(\frac{\Delta t^{2}}{1+\Delta t A\left(x_{i}, y_{j}\right)}\right) \frac{1}{\rho_{i, j}}\left[(\lambda+2 \mu)\left(\frac{\left(u_{2}\right)_{i+1, j}^{n}-2\left(u_{2}\right)_{i, j}^{n}+\left(u_{2}\right)_{i-1, j}^{n}}{\Delta x^{2}}\right)\right. \\
+(\lambda+\mu) & \left(\frac{\left(u_{1}\right)_{i+1, j+1}^{n}-\left(u_{1}\right)_{i+1, j-1}^{n}-\left(u_{1}\right)_{i-1, j+1}^{n}+\left(u_{1}\right)_{i-1, j-1}^{n}}{4 \Delta x \Delta y}\right) \\
+\mu & \left(\frac{\left(u_{2}\right)_{i, j+1}^{n}-2\left(u_{2}\right)_{i, j}^{n}+\left(u_{2}\right)_{i, j-1}^{n}}{\Delta y^{2}}\right) .
\end{aligned}
$$

Assim como no caso da equação da onda acústica, a aplicação das condições de Cerjan e Sochacki demanda condições do tipo

$$
\begin{aligned}
& B_{1}\left(\begin{array}{l}
u_{1} \\
u_{2}
\end{array}\right)\left(x,-b-N_{y}, t\right)=0 \\
& B_{2}\left(\begin{array}{l}
u_{1} \\
u_{2}
\end{array}\right)\left(x, b+N_{y}, t\right)=0 \\
& B_{3}\left(\begin{array}{l}
u_{1} \\
u_{2}
\end{array}\right)\left(-M_{x}, y, t\right)=0 \\
& B_{4}\left(\begin{array}{l}
u_{1} \\
u_{2}
\end{array}\right)\left(a+M_{x}, y, t\right)=0
\end{aligned}
$$

para o caso da onda elástica e, novamente, tais condições podem ser de Dirichlet, Neumann ou de absorção.

De acordo com o desenvolvimento apresentado por Engquist e Majda em [EM79], as condições de contorno A1 e A2 para a equação da onda elástica na formulação de deslocamento é dada pelas seguintes equações, referentes respectivamente à primeira e à segunda aproximações descritas na seção 2.2.1, para a borda direita do domínio:

$$
\left(\begin{array}{l}
\frac{\partial u_{1}}{\partial x} \\
\frac{\partial u_{2}}{\partial x}
\end{array}\right)+\left(\begin{array}{cc}
\frac{1}{\beta} & 0 \\
0 & \frac{1}{\alpha}
\end{array}\right) \cdot\left(\begin{array}{l}
\frac{\partial u_{1}}{\partial t} \\
\frac{\partial u_{2}}{\partial t}
\end{array}\right)=0
$$


$\left(\begin{array}{l}\frac{\partial^{2} u_{1}}{\partial t \partial x} \\ \frac{\partial^{2} u_{2}}{\partial t \partial x}\end{array}\right)+\left(\begin{array}{cc}\frac{1}{\beta} & 0 \\ 0 & \frac{1}{\alpha}\end{array}\right) \cdot\left(\begin{array}{c}\frac{\partial^{2} u_{1}}{\partial t^{2}} \\ \frac{\partial^{2} u_{2}}{\partial t^{2}}\end{array}\right)+\left(\begin{array}{cc}0 & \frac{\beta-\alpha}{\beta} \\ \frac{\beta-\alpha}{\alpha} & 0\end{array}\right) \cdot\left(\begin{array}{l}\frac{\partial^{2} u_{1}}{\partial t \partial y} \\ \frac{\partial^{2} u_{2}}{\partial t \partial y}\end{array}\right)+\left(\begin{array}{cc}\frac{\beta-2 \alpha}{2} & 0 \\ 0 & \frac{\alpha-2 \beta}{2}\end{array}\right) \cdot\left(\begin{array}{l}\frac{\partial^{2} u_{1}}{\partial y^{2}} \\ \frac{\partial^{2} u_{2}}{\partial y^{2}}\end{array}\right)=0$.

Para tais condições, são sugeridos ainda, em [EM77] e [CE77], os operadores de discretização

$$
\begin{aligned}
\left(\begin{array}{c}
D_{-}^{x}\left[\left(u_{1}\right)_{N, k}^{n}+\left(u_{1}\right)_{N, k}^{n+1}\right] \\
D_{-}^{x}\left[\left(u_{2}\right)_{N, k}^{n}+\left(u_{2}\right)_{N, k}^{n+1}\right]
\end{array}\right)+\left(\begin{array}{cc}
\frac{1}{\beta} & 0 \\
0 & \frac{1}{\alpha}
\end{array}\right) \cdot\left(\begin{array}{c}
D_{+}^{t}\left[\left(u_{1}\right)_{N, k}^{n}+\left(u_{1}\right)_{N-1, k}^{n}\right] \\
D_{+}^{t}\left[\left(u_{2}\right)_{N, k}^{n}+\left(u_{2}\right)_{N-1, k}^{n}\right]
\end{array}\right)=0 \\
\left(\begin{array}{c}
D_{0}^{t} D_{-}^{x}\left(u_{1}\right)_{N, k}^{n} \\
D_{0}^{t} D_{-}^{x}\left(u_{2}\right)_{N, k}^{n}
\end{array}\right)+\frac{1}{2}\left(\begin{array}{cc}
\frac{1}{\beta} & 0 \\
0 & \frac{1}{\alpha}
\end{array}\right) \cdot\left(\begin{array}{c}
D_{+}^{t} D_{-}^{t}\left[\left(u_{1}\right)_{N, k}^{n}+\left(u_{1}\right)_{N-1, k}^{n}\right] \\
D_{+}^{t} D_{-}^{t}\left[\left(u_{2}\right)_{N, k}^{n}+\left(u_{2}\right)_{N-1, k}^{n}\right]
\end{array}\right) \\
+\frac{1}{2}\left(\begin{array}{cc}
0 & \frac{\beta-\alpha}{\beta} \\
\frac{\beta-\alpha}{\alpha} & 0
\end{array}\right) \cdot\left(\begin{array}{c}
\left(D_{+}^{t} D_{0}^{y}\right)\left[\left(u_{1}\right)_{N, k}^{n-1}+\left(u_{1}\right)_{N-1, k}^{n}\right] \\
\left(D_{+}^{t} D_{0}^{y}\right)\left[\left(u_{2}\right)_{N, k}^{n-1}+\left(u_{2}\right)_{N-1, k}^{n}\right]
\end{array}\right) \\
+\frac{1}{2}\left(\begin{array}{cc}
\frac{\beta-2 \alpha}{2} & 0 \\
0 & \frac{\alpha-2 \beta}{2}
\end{array}\right) \cdot\left(\begin{array}{c}
\left(D_{+}^{y} D_{-}^{y}\right)\left[\left(u_{1}\right)_{N, k}^{n-1}+\left(u_{1}\right)_{N-1, k}^{n+1}\right] \\
\left(D_{+}^{y} D_{-}^{y}\right)\left[\left(u_{2}\right)_{N, k}^{n-1}+\left(u_{2}\right)_{N-1, k}^{n+1}\right]
\end{array}\right)=0,
\end{aligned}
$$

onde $D_{-}, D_{+}$e $D_{0}$ denotam os operadores de diferenças progressivas, regressivas e centrais, respectivamente.

Para a condição de Higdon, desenvolvida em [Hig90] e [Hig91], observemos, primeiramente, a condição de contorno

$$
\begin{aligned}
& \left(\frac{\partial}{\partial t}-v_{p} \frac{\partial}{\partial x}\right) u_{1}=0 \\
& \left(\frac{\partial}{\partial t}-v_{s} \frac{\partial}{\partial x}\right) u_{2}=0
\end{aligned}
$$

Essa forma decorre do fato de que, para ondas planas se propagando a um ângulo normal em direção ao contorno $x=0$, o deslocamento $u_{1}$ transporta as ondas $\mathrm{P}$, enquanto $u_{2}$ transporta as ondas S. Como é possível observar, trata-se de uma modificação da condição de contorno proposta por Clayton e Engquist.

O desenvolvimento da condição de Higdon para a equação da onda elástica é semelhante ao realizado para a equação (1.12). Ao longo do contorno, aplica-se um operador a cada componente do deslocamento e é possível, inclusive, adotar operadores distintos. Deve-se considerar que, diferentemente do caso de (1.12), a equação da onda elástica conta com duas velocidades de onda, correspondentes às ondas $\mathrm{P}$ e S.

A condição de Clayton e Engquist não absorve totalmente as ondas que se propagam a ângulos diferentes do normal. Por esse motivo, sugere-se a aplicação do seguinte operador a cada componente do deslocamento:

$$
\left[\left(\cos \alpha_{p}\right) \frac{\partial}{\partial t}-v_{p} \frac{\partial}{\partial x}\right]\left[\left(\cos \alpha_{s}\right) \frac{\partial}{\partial t}-v_{s} \frac{\partial}{\partial x}\right] .
$$

Novamente, os parâmetros $\alpha_{p}$ e $\alpha_{s}$ podem ser ajustados de modo a reduzir a reflexão. Embora $\mathrm{o}$ primeiro e o segundo fator estejam associados às ondas $\mathrm{P}$ e $\mathrm{S}$ respectivamente, também ajudam a absorver o outro tipo de onda. Se o primeiro fator for multiplicado por $\frac{v_{s}}{v_{p}}$, temos

$$
\left[\frac{v_{s}}{v_{p}}\left(\cos \alpha_{p}\right) \frac{\partial}{\partial t}-v_{s} \frac{\partial}{\partial x}\right]\left[\left(\cos \alpha_{s}\right) \frac{\partial}{\partial t}-v_{s} \frac{\partial}{\partial x}\right] \text {. }
$$

Por construção, esse operador gera uma condição de contorno que deve ser capaz de fornecer 
absorção completa de ondas $\mathrm{S}$ incidindo a ângulos iguais a $\Theta= \pm \cos ^{-1}\left(\frac{v_{s}}{v_{p}} \cos \alpha_{p}\right)$. Para ondas $\mathrm{P}$, pode ser realizado um procedimento análogo no segundo fator de (A.21).

Uma condição mais geral é dada pela aplicação do seguinte operador a cada componente do deslocamento:

$$
\prod_{j=1}^{p}\left(\beta_{j} \frac{\partial}{\partial t}-v_{p} \frac{\partial}{\partial x}\right) .
$$

Se cada $\beta_{j}$ tiver valor absoluto menor que ou igual a 1 , a condição é mais eficiente para absorção de ondas P. Se $\beta_{j} \approx \frac{v_{p}}{v_{s}}$, a condição estará voltada para a absorção de ondas S.

Para a obtenção da PML para o problema elastodinâmico, tratado em [CT01], consideramos a formulação velocidade-estresse. Escrevendo-a em forma matricial, temos

$$
\begin{gathered}
\varrho \frac{\partial}{\partial t} \mathbf{v}=D^{\|} \frac{\partial}{\partial y} \boldsymbol{\sigma}+D^{\perp} \frac{\partial}{\partial x} \boldsymbol{\sigma} \\
\frac{\partial}{\partial t} \boldsymbol{\sigma}=E^{\| l} \frac{\partial}{\partial y} \mathbf{v}+E^{\perp} \frac{\partial}{\partial x} \mathbf{v}, \\
D^{\|}=\left(\begin{array}{ccc}
0 & 0 & 1 \\
0 & 1 & 0
\end{array}\right), D^{\perp}=\left(\begin{array}{ccc}
1 & 0 & 0 \\
0 & 0 & 1
\end{array}\right) \\
E^{\|}=\left(\begin{array}{cc}
0 & 0 \\
0 & 1 \\
\frac{1}{2} & 0
\end{array}\right), E^{\perp}=\left(\begin{array}{cc}
1 & 0 \\
0 & 0 \\
0 & \frac{1}{2}
\end{array}\right) .
\end{gathered}
$$

Por simplicidade, omitimos $f(\mathbf{x}, t)$, uma vez que, em geral, essa função será identicamente nula na extensão do domínio. Para o sistema dado, a PML é formulada da forma

$$
\begin{aligned}
& \mathbf{v}=\mathbf{v}^{\|}+\mathbf{v}^{\perp} \\
& \varrho \frac{\partial}{\partial t} \mathbf{v}^{\|}=D^{\|} \frac{\partial}{\partial y} \boldsymbol{\sigma} \\
& \varrho \frac{\partial}{\partial t} \mathbf{v}^{\perp}+d(x) \mathbf{v}^{\perp}=D^{\perp} \frac{\partial}{\partial x} \boldsymbol{\sigma} \\
& \boldsymbol{\sigma}=\boldsymbol{\sigma}^{\|}+\boldsymbol{\sigma}^{\perp} \\
& \frac{\partial}{\partial t} \boldsymbol{\sigma}^{\|}=E^{\|} \frac{\partial}{\partial y} \mathbf{v} \\
& \frac{\partial}{\partial t} \boldsymbol{\sigma}^{\perp}+d(x) \boldsymbol{\sigma}^{\perp}=E^{\perp} \frac{\partial}{\partial x} \mathbf{v} .
\end{aligned}
$$

Para o caso em que o meio é isotrópico, homogêneo e elástico, podemos formular o sistema de equações para a PML como segue: 


$$
\begin{aligned}
\mathbf{v}=\mathbf{v}^{\|}+\mathbf{v}^{\perp} ; & \boldsymbol{\sigma}=\boldsymbol{\sigma}^{\|}+\boldsymbol{\sigma}^{\perp} \\
\varrho\left(\frac{\partial}{\partial t}+d(x)\right) v_{x}^{\perp}=\frac{\partial}{\partial x} \sigma_{x x} ; & \varrho \frac{\partial}{\partial t} v_{x}^{\|}=\frac{\partial}{\partial y} \sigma_{x y} \\
\varrho\left(\frac{\partial}{\partial t}+d(x)\right) v_{y}^{\perp}=\frac{\partial}{\partial x} \sigma_{x y} ; & \varrho \frac{\partial}{\partial t} v_{y}^{\|}=\frac{\partial}{\partial y} \sigma_{y y} \\
\left(\frac{\partial}{\partial t}+d(x)\right) \sigma_{x x}^{\perp}=(\lambda+2 \mu) \frac{\partial}{\partial x} v_{x} ; & \frac{\partial}{\partial t} \sigma_{x x}^{\|}=\lambda \frac{\partial}{\partial y} v_{y} \\
\left(\frac{\partial}{\partial t}+d(x)\right) \sigma_{y y}^{\perp}=\lambda \frac{\partial}{\partial x} v_{x} ; & \frac{\partial}{\partial t} \sigma_{y y}^{\|}=(\lambda+2 \mu) \frac{\partial}{\partial y} v_{y} \\
\left(\frac{\partial}{\partial t}+d(x)\right) \sigma_{x y}^{\perp}=\mu \frac{\partial}{\partial x} v_{y} ; & \frac{\partial}{\partial t} \sigma_{x y}^{\|}=\mu \frac{\partial}{\partial y} v_{x} .
\end{aligned}
$$

A seguir, analisamos os coeficientes de reflexão para a PML correspondente à equação da onda elástica e verificamos a estabilidade do método para esse caso, cujo desenvolvimento é mais complexo por tratar da propagação de dois tipos de onda se deslocando a velocidades diferentes.

Para avaliar a eficácia deste método, consideremos as ondas planas que são soluções da equação da onda elástica em meios isotrópicos

$$
\begin{gathered}
U_{p}=A_{p} \overrightarrow{d_{p}} e^{i \omega v_{p}\left(t-\frac{x \cos \theta+y \operatorname{sen} \theta}{v_{p}}\right)} \\
U_{s}=A_{s} \overrightarrow{d_{s}} e^{i \omega v_{s}\left(t-\frac{x \cos \theta+y \operatorname{sen} \theta}{v_{s}}\right)},
\end{gathered}
$$

onde $v_{p}$ e $v_{s}$ são as velocidades das ondas $\mathrm{P}$ e $\mathrm{S}$, respectivamente, $\theta$ é o ângulo de propagação da onda, $\overrightarrow{d_{p}}$ e $\overrightarrow{d_{s}}$ são as direções do movimento de uma partícula e $A_{p}$ e $A_{s}$ são as amplitudes das ondas.

Introduzimos um contorno em $x=\delta$, com uma condição de Dirichlet $\vec{v}=0$ e consideramos uma onda plana incidente nesse contorno denotada por

$$
U_{p}^{i n c}=A_{i n c} \overrightarrow{d_{p}} e^{i \omega v_{p}\left(t-\frac{x \cos \theta+y \operatorname{sen} \theta}{v_{p}}\right)} .
$$

Ao atingir o contorno, essa onda é refletida em uma onda $\mathrm{P}$ e uma onda $\mathrm{S}$, dadas por

$$
\begin{aligned}
U_{p p}^{r} & =A_{p}^{r} \overrightarrow{d_{p}^{r}} e^{i \omega v_{p}\left(t-\frac{-x \cos \theta_{1}+y \operatorname{sen} \theta_{1}}{v_{p}}\right)}, \\
U_{p s}^{r} & =A_{s}^{r} \overrightarrow{d_{s}^{r}} e^{i \omega v_{s}\left(t-\frac{x \cos \theta_{2}-y \operatorname{sen} \theta_{2}}{v_{s}}\right)} .
\end{aligned}
$$

Aqui, $\overrightarrow{d_{p}^{r}}=\left(-\cos \theta_{1}, \operatorname{sen} \theta_{1}\right)$ e $\overrightarrow{d_{s}^{r}}=\left(-\operatorname{sen} \theta_{2}, \cos \theta_{2}\right)$.

Procedendo de modo análogo para as ondas $\mathrm{S}$, obtemos também ondas $\mathrm{P}$ e $\mathrm{S}$, resultantes da reflexão causada pelo encontro da onda com o contorno inserido. Assim, podemos considerar os coeficientes de reflexão das ondas P e S, dados por

$$
R_{p p}=\frac{\left\|U_{p}^{r}\right\|}{\left\|U_{p}^{i n c}\right\|}, R_{p s}=\frac{\left\|U_{s}^{r}\right\|}{\left\|U_{p}^{i n c}\right\|}, R_{s p}=\frac{\left\|U_{p}^{r}\right\|}{\left\|U_{s}^{i n c}\right\|}, R_{s s}=\frac{\left\|U_{s}^{r}\right\|}{\left\|U_{s}^{\text {inc }}\right\|} .
$$

Para as ondas $\mathrm{P}$, temos 


$$
R_{p p}=\frac{\cos \left(\theta-\theta_{2}\right)}{\cos \left(\theta+\theta_{2}\right)}, R_{p s}=\frac{\operatorname{sen} \theta_{2}}{\cos \left(\theta+\theta_{2}\right)}
$$

Já para as ondas $\mathrm{S}$,

$$
R_{s p}=\frac{\operatorname{sen} \theta_{2}}{\operatorname{sen}\left(\theta-\theta_{2}\right)}, R_{s s}=\frac{\operatorname{sen}\left(\theta+\theta_{2}\right)}{\operatorname{sen}\left(\theta-\theta_{2}\right)} .
$$

Por construção, a onda não é refletida ao atingir a PML, camada na qual realizamos a mudança de coordenadas $\bar{x}_{j}(x)=x-\frac{i}{\omega v_{j}} \int_{0}^{x} d(s) d s$, com $j=p, s$ de modo que, após se deslocar por um comprimento $\delta$, a solução tem o seguinte valor, dado por $\bar{U}_{p}$ e $\bar{U}_{s}$ :

$$
\begin{aligned}
& \bar{U}_{p}=A_{p} \overrightarrow{d_{p}} e^{i \omega v_{p}\left[t-\frac{\cos \theta \cdot\left(\delta-\frac{i}{\omega v_{p}} \int_{0}^{\delta} d(s) d s\right)+\operatorname{sen} \theta \cdot y}{v_{p}}\right]}=U_{p} e^{-\frac{\cos \theta}{v_{p}} \int_{0}^{\delta} d(s) d s} . \\
& \bar{U}_{s}=A_{s} \overrightarrow{d_{s}} e^{i \omega v_{s}\left[t-\frac{\cos \theta \cdot\left(\delta-\frac{i}{\omega v_{s}} \int_{0}^{\delta} d(s) d s\right)+\operatorname{sen} \theta \cdot y}{v_{s}}\right]}=U_{s} e^{-\frac{\cos \theta}{v_{s}} \int_{0}^{\delta} d(s) d s .}
\end{aligned}
$$

As ondas $\bar{U}_{p}$ e $\bar{U}_{s}$ serão refletidas ao atingirem o contorno $x=\delta$, gerando ondas $\bar{U}_{p p}, \bar{U}_{p s}$, $\bar{U}_{s p}$ e $\bar{U}_{s s}$. Assim, os coeficientes de reflexão serão dados pela razão entre o valor absoluto da onda amortecida refletida e o valor absoluto da onda original, ambos em $x=\delta$. Desse modo, obtemos:

$$
\begin{aligned}
& R_{p p}^{\delta}=\frac{\left\|\bar{U}_{p p}^{r}\right\|}{\left\|U_{p}\right\|}=R_{p p} e^{-2 \frac{\cos \theta}{v_{p}} \int_{0}^{\delta} d(s) d s} \\
& R_{p s}^{\delta}=\frac{\left\|\bar{U}_{p s}^{r}\right\|}{\left\|U_{p}\right\|}=R_{p s} e^{-2 \frac{\cos \theta}{v_{p}} \int_{0}^{\delta} d(s) d s} \\
& R_{s p}^{\delta}=\frac{\left\|\bar{U}_{s p}^{r}\right\|}{\left\|U_{s}\right\|}=R_{s p} e^{-2 \frac{\cos \theta}{v_{s}} \int_{0}^{\delta} d(s) d s} \\
& R_{s s}^{\delta}=\frac{\left\|\bar{U}_{s s}^{r}\right\|}{\left\|U_{s}\right\|}=R_{s s} e^{-2 \frac{\cos \theta}{v_{s}} \int_{0}^{\delta} d(s) d s} .
\end{aligned}
$$

Questões relacionadas à estabilidade da PML para a equação da onda elástica são abordadas por diversos autores, como Duru e Kreiss [DK14] e Bécache, Fauqueux e Joly [BFJ03], de acordo com o qual apresentamos os resultados a seguir.

A relação de dispersão da equação da onda elástica nos permite afirmar que, para os valores de $\omega, k_{x}$ e $k_{y}$ para os quais $u=e^{i\left(k_{x}+k_{y} y-\omega t\right)}$ é uma solução de (A.8), pelo menos uma das relações abaixo é satisfeita:

$$
\omega^{2}=v_{p}^{2}\left(k_{x}^{2}+k_{y}^{2}\right), \omega^{2}=v_{s}^{2}\left(k_{x}^{2}+k_{y}^{2}\right) .
$$

Definimos a função $F(\omega, k)$ como segue:

$$
F(\omega, k)=\omega^{2}-c_{i}\left(k_{x}^{2}+k_{y}^{2}\right)
$$

Sejam $\omega_{p}$ e $\omega_{s}$ os valores de $\omega$ para os quais a primeira e a segunda condições dadas são satisfeitas respectivamente. Se $\omega=\omega_{p}, \omega_{s}$ e $k=\left(k_{x}, k_{y}\right)$, o diagrama de lentidão é definido como o conjunto de $\vec{S}=(\omega, k)$ tal que $F\left(1, \frac{k}{\omega}\right)=0$. Definindo ainda a velocidade de grupo como $V_{g}(k)=\nabla_{k} \omega(k)$, temos o seguinte resultado: se o sistema original é estritamente e fortemente hiperbólico e tal que todos seus autovalores sejam diferentes de 0 para $k \neq 0$, o sistema da PML será estável em altas frequências se, e somente se, $\forall k$ tal que $|k|=1, S_{1}(k) \cdot V_{g_{1}}(k) \geq 0$. Este critério é conhecido como condição de estabilidade geométrica e pode ser aplicado inclusive para meios anisotrópicos. 
Para meios ortotrópicos, a matriz $C$ que relaciona os tensores de estresse e de tensão tem a forma

$$
C=\left[\begin{array}{ccc}
c_{11} & c_{12} & 0 \\
c_{12} & c_{22} & 0 \\
0 & 0 & c_{33}
\end{array}\right]
$$

Para esse caso, a condição de estabilidade geométrica equivale a

$$
\left\{\left(c_{12}+c_{33}\right)^{2}-c_{11}\left(c_{22}-c_{33}\right)\right\} \cdot\left\{\left(c_{12}+c_{33}\right)^{2}+c_{33}\left(c_{22}-c_{33}\right)\right\} \leq 0 .
$$

Como o caso isotrópico equivale ao ortotrópico com $c_{11}=c_{22}=\lambda+2 \mu, c_{12}=\lambda$ e $c_{33}=\mu$, podemos verificar que a condição acima é satisfeita. De fato,

$$
\left\{(\lambda+\mu)^{2}-(\lambda+2 \mu)(\lambda+2 \mu-\mu)\right\} \cdot\left\{(\lambda+\mu)^{2}+\mu(\lambda+2 \mu-\mu)\right\}=(\lambda+\mu)^{2}(-\mu)(\lambda+2 \mu)<0 .
$$

Portanto, a estabilidade em altas frequências é garantida para o meio isotrópico.

O desenvolvimento da PML para contornos mais gerais também tem como base a escrita da equação da onda elástica na formulação velocidade-estresse no domínio da frequência, da seguinte forma:

$$
\begin{aligned}
& i \omega \rho \mathbf{v}=\hat{n} \partial_{n} \cdot \boldsymbol{\sigma}+\nabla^{\|} \\
& i \omega \boldsymbol{\sigma}=\mathbf{C}: \hat{n} \partial_{n} \mathbf{v}+\mathbf{C}: \nabla^{\|} .
\end{aligned}
$$

Define-se, então, uma nova coordenada complexa $\tilde{n}$ tal que

$$
\tilde{n}(n)=n-\frac{i}{\omega} \int_{0}^{n} d(s) d s \Rightarrow \frac{\partial n}{\partial \tilde{n}}=\frac{i \omega}{i \omega+d(n)}
$$

e, a partir daí, o raciocínio aplicado é análogo ao apresentado anteriormente. O desenvolvimento completo pode ser encontrado no trabalho de Komatitsch e Tromp [KT03].

Por fim, a HABC para a equação da onda elástica pode ser aplicada separadamente a cada componente do deslocamento, uma vez que as condições de absorção aqui abordadas são utilizadas dessa forma. Assim, calcula-se a solução a partir da equação original (nesse caso, adota-se a formulação de deslocamento) e também a solução fornecida por uma condição de absorção como as apresentadas anteriormente, realizando, em seguida, a média ponderada entre as duas soluções obtidas conforme descrito na seção 2.3.4. 
APÊNDICE A 


\section{Referências Bibliográficas}

[AB19] Paula N de Araújo e Saulo R M Barros. Comparison of absorbing boundary conditions for acoustic wave equations. STMI 2019 - Workshop on Software Technology for Modeling and Inversion, 2019. 3

[Bat13] Markus Bath. Introduction to seismology, volume 27. Birkhäuser, 2013. 77

[Ber94] Jean-Pierre Berenger. A perfectly matched layer for the absorption of electromagnetic waves. Journal of computational physics, 114(2):185-200, 1994. 2

[Bér15] Jean-Pierre Bérenger. A historical review of the absorbing boundary conditions for electromagnetics. Em Forum for Electromagnetic Research Methods and Application Technologies, volume 9, 2015. 2

[BFJ03] Eliane Bécache, Sandrine Fauqueux e Patrick Joly. Stability of perfectly matched layers, group velocities and anisotropic waves. Journal of Computational Physics, 188(2):399-433, 2003. 88

[BWZ10] Moysey Brio, Gary M Webb e Aramais R Zakharian. Numerical time-dependent partial differential equations for scientists and engineers, volume 213. Academic Press, 2010. 7

[CE77] Robert Clayton e Björn Engquist. Absorbing boundary conditions for acoustic and elastic wave equations. Bulletin of the seismological society of America, 67(6):15291540, 1977. 85

[CFL28] Richard Courant, Kurt Friedrichs e Hans Lewy. Über die partiellen differenzengleichungen der mathematischen physik. Mathematische annalen, 100(1):32-74, 1928. 11

[Cha04] Chris Chapman. Fundamentals of seismic wave propagation. Cambridge university press, 2004. 82

[Che19] Albert Chern. A reflectionless discrete perfectly matched layer. Journal of Computational Physics, 381:91-109, 2019. 3

[CKKR85] Charles Cerjan, Dan Kosloff, Ronnie Kosloff e Moshe Reshef. A nonreflecting boundary condition for discrete acoustic and elastic wave equations. Geophysics, 50(4):705-708, 1985. 2,21

[CT01] Francis Collino e Chrysoula Tsogka. Application of the perfectly matched absorbing layer model to the linear elastodynamic problem in anisotropic heterogeneous media. Geophysics, 66(1):294-307, 2001. 86

[CW94] Weng Cho Chew e William H Weedon. A 3d perfectly matched medium from modified maxwell's equations with stretched coordinates. Microwave and optical technology letters, 7(13):599-604, 1994. 3 
[DK14] Kenneth Duru e Gunilla Kreiss. Boundary waves and stability of the perfectly matched layer for the two space dimensional elastic wave equation in second order form. SIAM Journal on Numerical Analysis, 52(6):2883-2904, 2014. 88

[DKK15] Kenneth Duru, Jeremy E Kozdon e Gunilla Kreiss. Boundary conditions and stability of a perfectly matched layer for the elastic wave equation in first order form. Journal of Computational Physics, 303:372-395, 2015. 3

[EM77] Björn Engquist e Andrew Majda. Absorbing boundary conditions for the numerical simulation of waves. Proceedings of the National Academy of Sciences, 74(5):1765-1766, 1977. $2,15,17,85$

[EM79] Bjorn Engquist e Andrew Majda. Radiation boundary conditions for acoustic and elastic wave calculations. Communications on pure and applied mathematics, 32(3):313357, 1979. 17, 84

[Eva10] L.C. Evans. Partial Differential Equations. Graduate studies in mathematics. American Mathematical Society, 2010. 7

[Fic10] Andreas Fichtner. Full seismic waveform modelling and inversion. Springer Science \& Business Media, 2010. 2, 3, 23

[FN03] Gaetano Festa e Stefan Nielsen. Pml absorbing boundaries. Bulletin of the Seismological Society of America, 93(2):891-903, 2003. 3

[GKO95] Bertil Gustafsson, Heinz-Otto Kreiss e Joseph Oliger. Time dependent problems and difference methods, volume 24. John Wiley \& Sons, 1995. 7, 8

[GS10] Marcus J Grote e Imbo Sim. Efficient pml for the wave equation. arXiv preprint arXiv:1001.0319, 2010. 23, 27, 29

[GSZY17] Yingjie Gao, Hanjie Song, Jinhai Zhang e Zhenxing Yao. Comparison of artificial absorbing boundaries for acoustic wave equation modelling. Exploration Geophysics, 48(1):76-93, 2017. 50

[Hig86] Robert L Higdon. Absorbing boundary conditions for difference approximations to the multidimensional wave equation. Mathematics of computation, 47(176):437-459, 1986. 2

[Hig87] Robert L Higdon. Numerical absorbing boundary conditions for the wave equation. Mathematics of computation, 49(179):65-90, 1987. 15, 19

[Hig90] Robert L Higdon. Radiation boundary conditions for elastic wave propagation. SIAM Journal on Numerical Analysis, 27(4):831-869, 1990. 19, 85

[Hig91] Robert L Higdon. Absorbing boundary conditions for elastic waves. Geophysics, 56(2):231-241, 1991. 19, 85

[Hol77] Richard Holland. Threde: A free-field emp coupling and scattering code. IEEE Transactions on Nuclear Science, 24(6):2416-2421, 1977. 2

[Joh82] Fritz John. Partial differential equations. Springer-Verlag New York, 1982. 7

[Joh08] Steven G Johnson. Notes on perfectly matched layers (pmls). Lecture notes, Massachusetts Institute of Technology, Massachusetts, 29, 2008. 23

[Key85] R Gh Keys. Absorbing boundary conditions for acoustic media. Geophysics, 50(6):892902, 1985. 2 
[Kim19] Dojin Kim. A modified pml acoustic wave equation. Symmetry, 11(2):177, 2019. 3

[KMG09] Jozef Kristek, Peter Moczo e Martin Galis. A brief summary of some pml formulations and discretizations for the velocity-stress equation of seismic motion. Studia Geophysica et Geodaetica, 53(4):459, 2009. 3

[KT03] Dimitri Komatitsch e Jeroen Tromp. A perfectly matched layer absorbing boundary condition for the second-order seismic wave equation. Geophysical Journal International, 154(1):146-153, 2003. 23, 89

[LHYY84] Zhen-Feng LIAO, Kong-liang Huang, Bai-po Yang e Yi-Fan YUAN. A transmitting boundary for transient wave analyses. Science in China Series A-Mathematics, Physics, Astronomy \& Technological Science, 27(10):1063-1076, 1984. 2

[LL11] Hans Petter Langtangen e Hans Petter Langtangen. A primer on scientific programming with Python, volume 6. Springer, 2011. 33

[LL17] Hans Petter Langtangen e Svein Linge. Finite Difference Computing with PDEs - A Modern Software Approach. Springer Open, 2017. 33

[LS10] Yang Liu e Mrinal K Sen. A hybrid scheme for absorbing edge reflections in numerical modeling of wave propagation. Geophysics, 75(2):A1-A6, 2010. 3, 30

[LS18] Yang Liu e Mrinal K Sen. An improved hybrid absorbing boundary condition for wave equation modeling. Journal of Geophysics and Engineering, 15(6):2602-2613, 2018. 3, 31

[Mer71] David E Merewether. Transient currents induced on a metallic body of revolution by an electromagnetic pulse. IEEE Transactions on Electromagnetic Compatibility, (2):41-44, 1971. 2

[MKG ${ }^{+}$07] Peter Moczo, Jozef Kristek, M Galis, P Pazak e M Balazovjech. The finite-difference and finite-element modeling of seismic wave propagation and earthquake motion. Acta Physica Slovaca. Reviews and Tutorials, 57(2):177-406, 2007. 82

[PM10] Damir Pasalic e Ray McGarry. Convolutional perfectly matched layer for isotropic and anisotropic acoustic wave equations. Em SEG Technical Program Expanded Abstracts 2010, páginas 2925-2929. Society of Exploration Geophysicists, 2010. 3

[Rey78] Albert C Reynolds. Boundary conditions for the numerical solution of wave propagation problems. Geophysics, 43(6):1099-1110, 1978. 2

[RL13] Zhiming Ren e Yang Liu. A hybrid absorbing boundary condition for frequency-domain finite-difference modelling. Journal of Geophysics and Engineering, 10(5):054003, 2013. 30,31

[She09] Peter M Shearer. Introduction to seismology. Cambridge University Press, 2009. 78

[SKG $\left.{ }^{+} 87\right]$ James Sochacki, Robert Kubichek, John George, WR Fletcher e Scott Smithson. Absorbing boundary conditions and surface waves. Geophysics, 52(1):60-71, 1987. 2, 21

[Ste12] Ian Stewart. In pursuit of the unknown: 17 equations that changed the world. Basic Books, 2012. 1

[Str04] John C Strikwerda. Finite difference schemes and partial differential equations, volume 88. Siam, 2004. 12, 15 
[TC97] FL Teixeira e Weng Cho Chew. Systematic derivation of anisotropic pml absorbing media in cylindrical and spherical coordinates. IEEE microwave and guided wave letters, 7(11):371-373, 1997. 3

$\left[\mathrm{VECA}^{+} 12\right]$ Jean Virieux, Vincent Etienne, Victor Cruz-Atienza, Romain Brossier, Emmanuel Chaljub, Olivier Coutant, Stephane Garambois, Diego Mercerat, Vincent Prieux, Stephane Operto et al. Modelling seismic wave propagation for geophysical imaging. Masaki Kanao, 2012. 82 